\title{
Optical Properties of Substrate Supported Mixtures of Silver and Gold Nanoparticles
}

\author{
By \\ Devron Colley

\begin{abstract}
A thesis submitted to the Department of Chemistry
in partial fulfillment of the requirements for the degree of

Master of Science
\end{abstract}

To

Carleton University

Department of Chemistry

Ottawa, Ontario, Canada

(C) 2021, Devron Colley 
The undersigned hereby recommends to the

Department of Chemistry

acceptance of this thesis,

\title{
Optical Properties of Substrate Supported Mixtures of Silver and Gold Nanoparticles
}

\author{
submitted by: Devron Colley \\ in partial fulfillment of the requirements for the degree of \\ Master of Science
}

Dr. Anatoli Ianoul

(Supervisor) 


\section{Abstract:}

Metal nanoparticles, silver ( $\mathrm{Ag}$ ) and gold $(\mathrm{Au})$, have been extensively explored in the field of nanoscience owing to their strong plasmonic responses in the visible region of the electromagnetic spectrum. Herein, mixtures of nanoparticles (NPs) of different composition were combined to achieve new nanoarchitectures with interesting and useful electromagnetic properties. Thus, AuNPs and Ag nanocubes (NCs) were mixed and examined for their optical properties. It was presumed that these mixtures would produce materials with multiple plasmon modes across the visible spectrum providing opportunity for greater exploitation of the NP optical properties and general manipulation of light within one simple system. The spectral information obtained from individual depositions of NPs on substrates provided information on the light extinction exhibited by the nanomaterials. For AgNCs supported on a polystyrene (PS) thin film a dipolar resonance is observed at $439 \mathrm{~nm}$, and for AuNPs their dipolar resonance appeared at $530 \mathrm{~nm}$. The plasmonic modes obtained in the mixture are tunable across the UV-vis-NIR spectrum by manipulation of their local environment (e.g., NPs embedding into a thin polymer film). The substrates were heated conventionally and photothermally leading to nanoparticle embedment. Excitation of AgNCs at $458 \mathrm{~nm}$ and AuNPs at $568 \mathrm{~nm}$ leads to NP embedment, as these wavelengths are near the dipolar resonance. An employed use of this nanoarchitecture and its tunability, is the possibility to expand the plasmonic colouring regime through the creation of a gap mode. These resonances result in long-lasting, iridescent coloured materials with promising tunability. Specifically, the exploitation of the separation between the NPs and a thin metal mirror results in a variety of colours. This work shows that mixtures of AuNPs and AgNCs can be synthesized, and their optical response can be exploited by embedding the nanoparticles into a polymer film. 


\section{Acknowledgments:}

I would like to thank Dr. Anatoli Ianoul for his guidance throughout my graduate studies.

Without his support, there would be no work to present. My experiences, knowledge, and desire to be an exceptional chemist are of his fruition. Many students have come and gone since my inception within the group, but Dr. Ianoul was always there offering a necessary sense of consistency. Being part of the Ianoul group has afforded me a bounty of information not known by even the common chemist and for that I am grateful.

Previous students in the group deserve acknowledgement as well. Specifically, Dr. Daniel Prezgot is thanked for his kinship and direction. Dr. Dan's extensive knowledge ability to impart said knowledge onto lowly students, such as myself, was greatly appreciated throughout my years with the Ianoul group. Ella Kirkland (another important comrade of mine) was one of the first scientists at Carleton to make me feel intelligent, and worthy of attaining a MSc. I am beyond grateful for the friendship we have established for without her I would not have grown to where I have. Alongside these graduate students came many undergraduates who have offered time to assist in experiment, and relief from life's stressors. Moarij Syed is identified here for his extensive preliminary work on AuNP synthesis and deposition onto substrates. I wish the best for all my colleagues of the past and present!

Life has not been easy throughout my studies, and the state of the world in the past few years has resulted in major alterations to the way my education was accessed. The support of my friends and family kept me afloat through these trying times. Without them I am not sure I could have made it through to the end. For all those who have aided me along this journey, I am truly grateful! 


\section{Table of Contents}

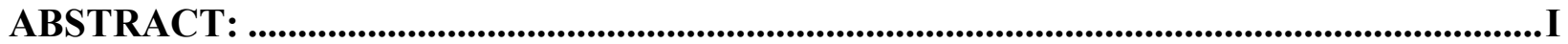

ACKNOWLEDGMENTS:........................................................................................................ II

LIST OF TABLES:

LIST OF FIGURES:

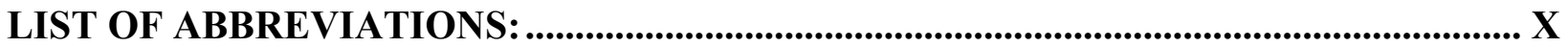

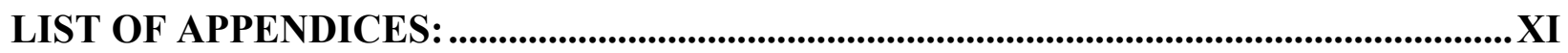

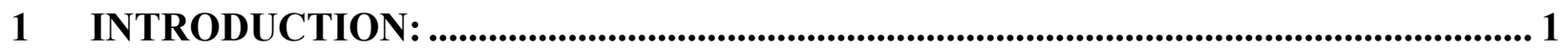

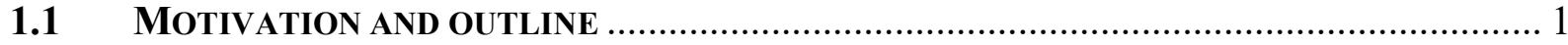

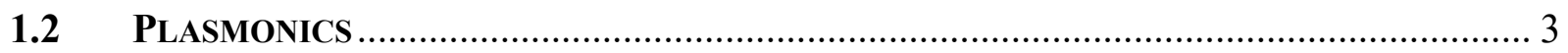

1.2.1 Light Interaction: Simple Plasmonic Models ……........................................... 5

1.2.2 Choice of Metal Nanostructures ……………................................................... 9

1.2.3 Shape and Size Effects on Plasmonic Materials ............................................ 10

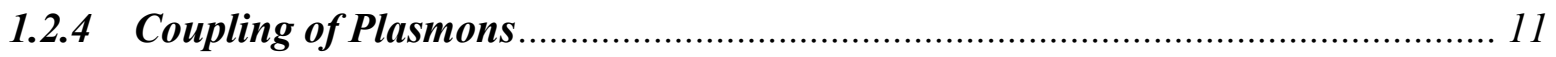

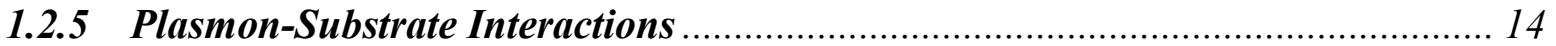

1.2.6 Gap Plasmons: Intentional Coupling of Surface Plasmon Resonances ............... 17

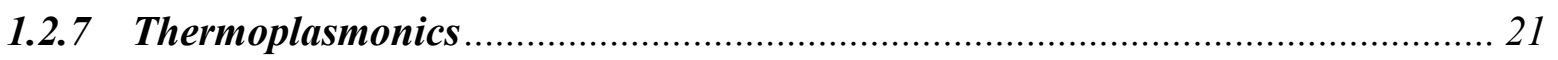

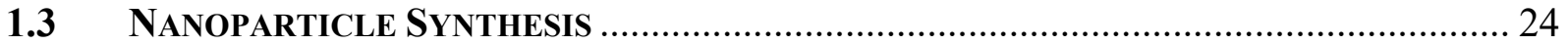

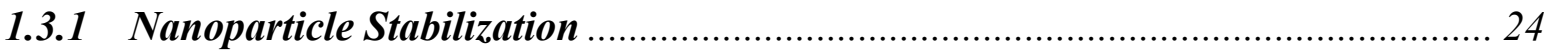

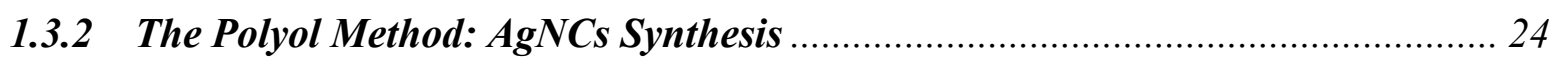

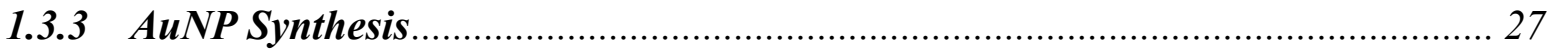

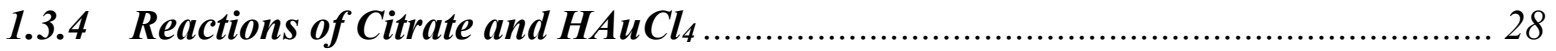

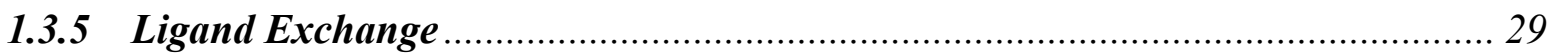

1.4 NANOMATERIAL ASSEMBLY ............................................................................. 30

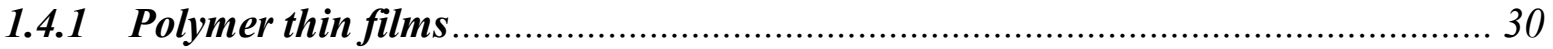

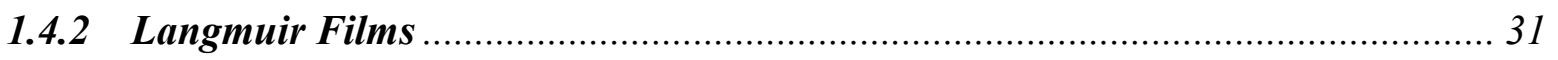

1.4.3 Nanoparticle Embedding into a Polymer Film ............................................... 33

1.5 Describing Plasmonic Systems and Materials.............................................. 35

1.5.1 Finite-Difference Time Domain (FDTD) Modelling ………............................ 35 


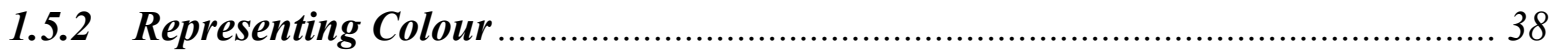

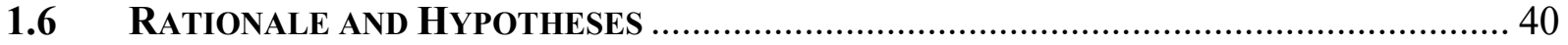

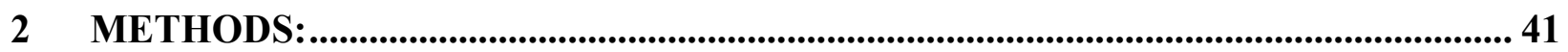

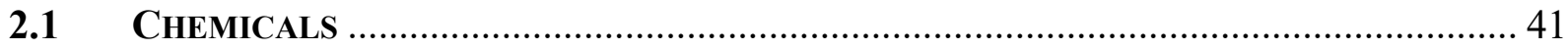

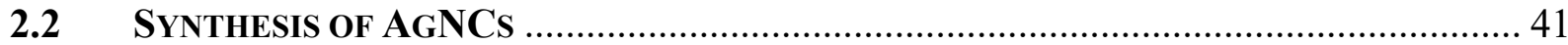

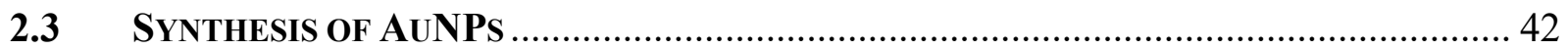

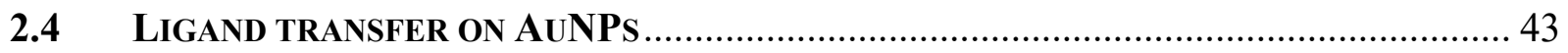

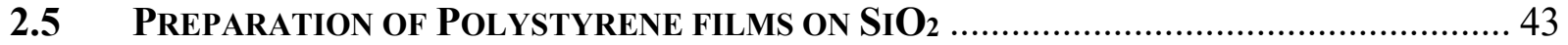

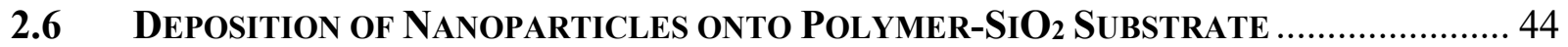

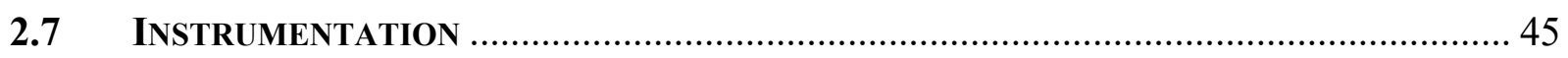

2.8 DETERMINATION OF 1:1 EXTINCTION MiXTURE OF AUNPS AND AgNCs ................ 45

2.9 Selective Embedment of The Nanoparticles into the Polymer Film......... 46

2.9.1 Description of Optical System.................................................................... 46

2.9.2 Determining Embedment Threshold ....................................................... 46

2.9.3 Investigating Selective Embedment................................................................. 46

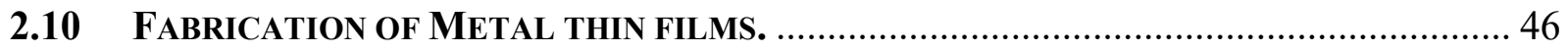

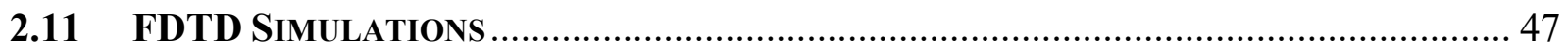

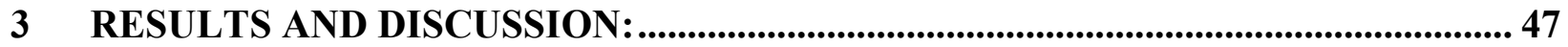

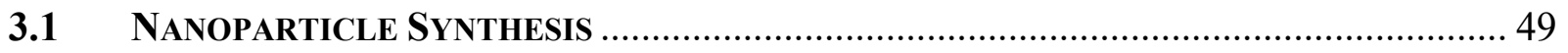

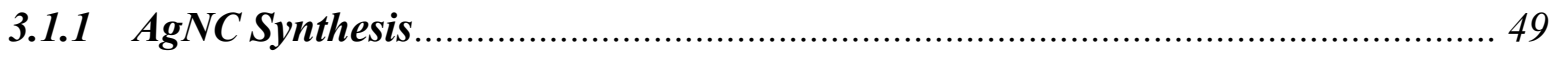

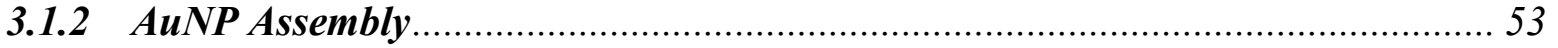

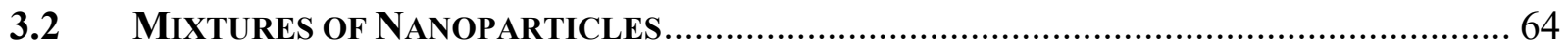

3.2.1 Determination of concentrations for 1:1 extinction mixture of AuNPs:AgNCs..... 64

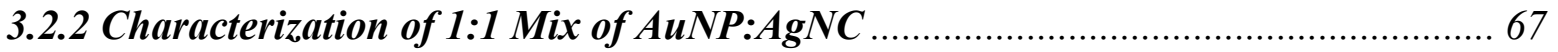

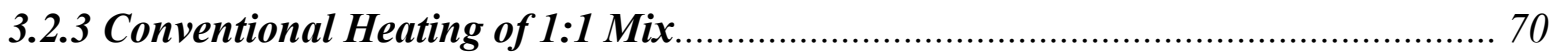

3.3 SPECTRALLY SELECTIVE ThERMOPLASMONIC EMBEDMENT ................................. 71

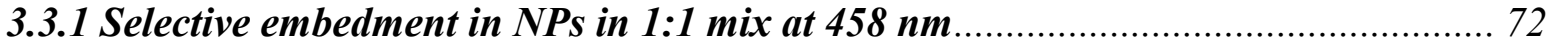

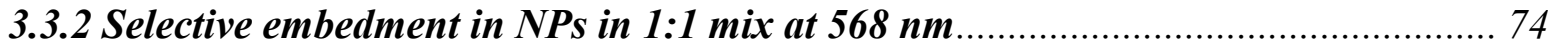

3.3.3 Selective embedment with switching laser from $568 \mathrm{~nm}$ to $458 \mathrm{~nm}$..................... 77

3.4 Gap Plasmons: New Nanoarchitectures for Colour Generation.............. 79 


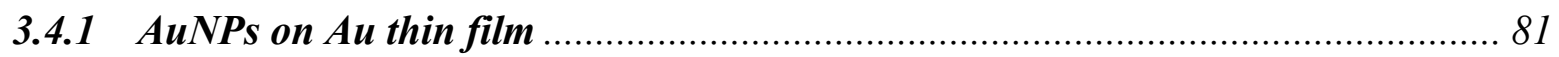

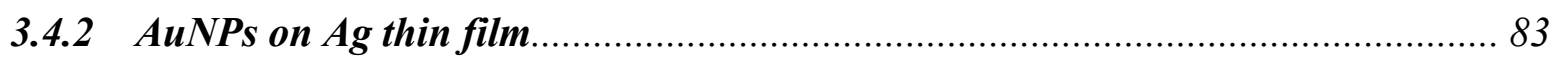

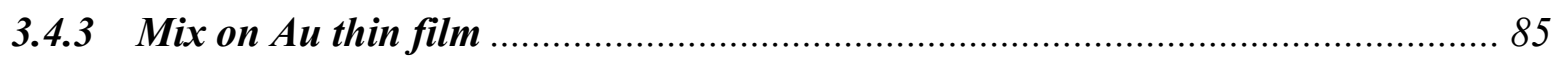

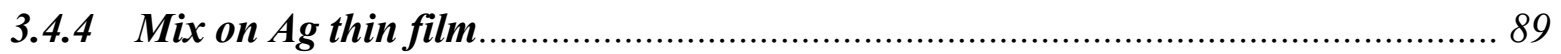

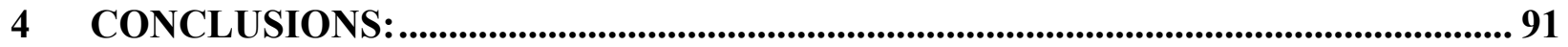

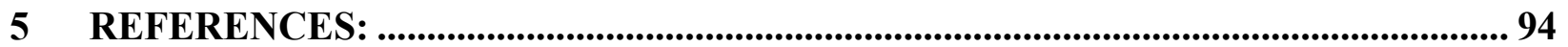

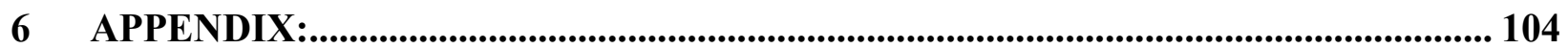




\section{List of Tables:}

Table 1: Calculated values for determination of 1:1 mix of AuNP:AgNC 65

\section{List of Figures:}

Figure 1: Schematic of the selective thermoplasmonic embedment of nanoparticles upon laser irradiance at $458 \mathrm{~nm}$, and $568 \mathrm{~nm}$.

Figure 2: Visualization of (a) LSP and (b) PSP. Reprinted with permission from ref (20)

Copyright 2011 American Chemical Society ....

Figure 3: Plots of the real (A) and imaginary (B) components of the dielectric function of Ag, $\mathrm{Au}$ and $\mathrm{Si}$ as a function of wavelength. Reproduced with permission from ref 20. Copyright 2011

ACS.

Figure 4: Quality factor (Q) of the LSPR of different metals at the metal/air interface. The shaded area indicates the region at which Q, and hence plasmonic response is strongest.

Reproduced with permission from ref 20. Copyright 2011 ACS ......................................... 9

Figure 5:Illustration of parallel and perpendicular coupling modes of a NP dimer .................. 11

Figure 6: Schematic of NP dipolar coupling using the hybridization model to give the low energy bonding mode and higher energy anti-bonding mode ............................................... 12

Figure 7: Schematic illustration of the classical regime $\left(\mathrm{d}_{\mathrm{CR}}\right)$, quantum regime $\left(\mathrm{d}_{\mathrm{QR}}\right)$, and conductive contact regime $\left(\mathrm{d}_{\mathrm{CC}}\right)$

Figure 8: Illustration (left) of substrate distance dependence of spherical NP coupling, FDTD simulated extinction spectra of a nanoparticle $100 \mathrm{~nm}$ above the dielectric substrate (Solid line), and on the substrate (Dashed line), with normal (to the substrate) (Dh, Red) and parallel (Dv, Blue) polarized light.

Figure 9: FDTD theory showing (A) the emergence of a second peak as a single nanocube (90$\mathrm{nm}$ diameter) approaches a dielectric substrate, and $(\mathrm{B}, \mathrm{C})$ the field intensities for peaks 1 and 2 of the nanocube in contact with the substrate (the white line in the field pattern images represent the substrate). Reprinted with permission from ref (57). Copyright 2013 ACS. 16

Figure 10: A) Image Interaction of Nanosphere with Metallic Substrate, B) Plasmon Hybridization Model, C) Perpendicular Dipole Mode, D) Parallel Dipole mode. Reprinted with permission from (59). Copyright (2004) American Chemical Society..................................... 18 Figure 11: Coupling of Nanosphere LSPR to SP on metallic surface with a thickness of a) 400$100 \mathrm{~nm}$, b) 25-16 nm, c) 12-4 nm. Reprinted with permission from (60). Copyright (2005) American Chemical Society.

Figure 12: a) Electric field intensities of $60 \mathrm{~nm} \mathrm{Ag}$ nanocubes over an Ag film separated by a dielectric spacer. The electric field intensity increases significantly when the particle is brought within $5 \mathrm{~nm}$ of the film. b) associated reflectance calculations showing a significant shift in the gap plasmon

Figure 13: Schematic representation of the lifetime of a plasmon resonance. Upon the plasmon's excitation (a) the excited carriers undergo Landau damping (b) forming a thermal population of hot carriers (c) which subsequently relax and produce heat (d) through collisions in the lattice. Reproduced with permission, (C) Springer Nature publishing group, 2015 ref 25 21

Figure 14: Spectra of the Joule numbers for a large set of materials. Materials have been arranged in families: Plasmonic resonance out of the visible range (left), in the visible range (middle), and the singular case of silver (right) where data have been plotted considering two 
distinct data sets (the optical constants of Johnson and Christy, and of Palik's handbook). Adapted with permission from (72). Copyright 2015 ACS ................................................... 23 Figure 15: Calculated Jo number plotted as a function of the resonance wavelength of ellipsoids made of various materials. Adapted with permission from (72). Copyright 2015 ACS 23 Figure 16: Shape-controlled synthesis of silver nanostructures. Ag nuclei will grow into either single crystals, or crystals containing single or multiple twin defects. Passivating agents can then be used to select the final morphology of the particle. Reproduced with permission from ref 81 . Copyright 2007 ACS. 25

Figure 17: LaMer diagram of the growth process of colloidal crystals. After reduction of the precursor to $\mathrm{M}^{0}$ the solution becomes super-saturated with $\mathrm{M}^{0}$ initiating self-nucleation of the particles at a critical concentration $\left(\mathrm{C}_{\text {crit }}\right)$. Subsequent growth and agglomeration of the particles Reprinted with permission from ref 72. Copyright 2013 RCS and adapted from ref 71 (Copyright ACS 1950). 26

Figure 18: Langmuir film substrate transfer technique: Blodgett deposition (left) and Schaefer deposition (right). Reproduced from 116 Copyright Elsevier 2006.

Figure 19: a) Schematic diagram of an electromagnetic wave showing the electric (E) and magnetic $(\mathrm{H})$ components and $\mathrm{b})$ a diagram of the Yee cell, where $\mathrm{E}(\mathrm{t})$ and $\mathrm{H}(\mathrm{t})$ are offset in half-steps to propagate fields. Reproduced from ref 125 under open access CC license 4.0...... 37 Figure 20: a) plot of the CIE 1931 colour space with, with the sRGB colour space shown within. Colours outside the triangle cannot be rendered properly on your display! b) The tristimulus colour matching functions, which model the sensitivity of the human eye to red, green, and blue light. ...

Figure 21: Monitoring the polyol synthesis using UV-vis. Inset TEM image of final AgNCs... 50 Figure 22: Extinction spectra of a) experimental AgNCs in solution (top) and on a PS substrate (bottom), and b) FDTD modeled scattering, absorption, and extinction cross-section of $60 \mathrm{~nm}$

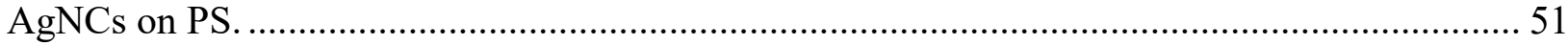

Figure 23: Respective transmittance and Reflectance spectra for AgNCs on PS.................... 52 Figure 24: Extinction spectra for the AuNP synthesis. AuNP growth from seed (G0) to final AuNPs (G10) with average particle size of $68 \mathrm{~nm}$ (left) and ligand transfer from citrate to PVP (right).

Figure 25: Experiments of Au NPs. a) final AuNP-PVP extinction spectrum $100 \mathrm{X}$ in EtOH, b) AFM image of a smooth PS film measuring $150 \mathrm{~nm}$ in height, c) spectrum of AuNPs-PVP on PS at different surface pressures, and d) Langmuir trough with AuNPs-PVP .............................. 55

Figure 26: AFM images of $1 \mathrm{mN} / \mathrm{m}$ AuNPs on PS. a) $10 \times 10 \mu \mathrm{m}$ and b) $5 \times 5 \mu \mathrm{m}$................... 57 Figure 27: FDTD model of extinction for $60 \mathrm{~nm}$ AuNPs embedding into PS over a depth interval of 5-60 nm.

Figure 28: Experimental results of extinction for AuNPs embedding into PS over a time interval of 0-20 min at $\sim 125^{\circ} \mathrm{C}$. Extinction spectra $(\mathrm{a}, \mathrm{b})$ and AFM images $(\mathrm{c}, \mathrm{d})$ of initial and final states; inset with the colour images in real-time and their colour representation based on CIE 1931 using their reflectance spectra. 62

Figure 29: Comparison of FDTD Model and Exp Results of AuNPs embedding into PS over a depth interval of 5-40 $\mathrm{nm}$..... 64

Figure 30: Experiments of 1:1 extinction (e) Mixtures of Au:Ag NPs. a) 100X dilution extinction spectrum of AuNPs, AgNCs and a 1:1 Mix, b) spectrum of 1:1 Mix deposited on 150 $\mathrm{nm}$ PS at increasing surface pressures, c) photograph of 100e NPs after 100X dilution, and d) Langmuir trough with 1:1 Mix. 
Figure 31: AFM data for a 1:1 Mix of Au:Ag NPs on PS $(1 \mathrm{mN} / \mathrm{m})$, inset spectrum of this slide (top). Bottom shows different types of dimers which occur in the sample.

Figure 32: FDTD Model spectra for an Au:Ag dimer at varying distances

Figure 33:AFM images of 1:1 Mix on PS $(2 \mathrm{mN} / \mathrm{m})$ a) before and $b$ ) after heating, inset CIE

1931 colour representation of these slides and c) Transmission, Reflectance and Absorptance

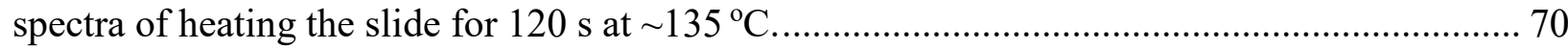

Figure 35: AFM images of 1:1 mix on PS after $458 \mathrm{~nm}$ laser exposure $(22 \mathrm{~mW})$ for a) $0 \mathrm{~s}, \mathrm{~b}) 15$ $\mathrm{s}$, c) $30 \mathrm{~s}$, and d) $55 \mathrm{~s}$.

Figure 36: AFM images and respective cross-sections of 1:1 mix on PS after $568 \mathrm{~nm}$ laser

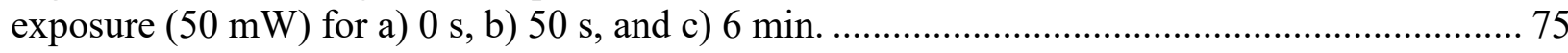

Figure 37: AFM images of 1:1 mix on PS after $568 \mathrm{~nm}$ laser exposure $(50 \mathrm{~mW})$ for a) $0 \mathrm{~s}, \mathrm{~b}) 15$ $\mathrm{s}$, and subsequent $458 \mathrm{~nm}$ laser exposure $(22 \mathrm{~mW})$ for c) $10 \mathrm{~s}$ and, d) $1 \mathrm{~min} . \ldots \ldots \ldots \ldots \ldots \ldots \ldots \ldots . . . . . . . . . . . . .78$ Figure 38: FDTD Model for Reflectance spectra of $60 \mathrm{~nm} \mathrm{AgNCs} \mathrm{on} 50 \mathrm{~nm} \mathrm{Au}$ (left), and 50 $\mathrm{nm} \mathrm{Ag}$ (right) with respect to a change in the gap distance (nm) by embedment into a $16 \mathrm{~nm}$, and $20 \mathrm{~nm}$ PS film respectively. 80

Figure 39: Reflectance spectra of the FDTD model of AuNPs on Au film with respect to a change in the gap distance $(\mathrm{nm})$. The CIE representation of the predicted colours is below and is a function of embedment depth $(0-20 \mathrm{~nm})$

Figure 40: Reflectance spectra of the Exp results of AuNPs on Au film with respect to a change in the gap distance $(\mathrm{nm})$......

Figure 41: Reflectance spectra of the FDTD model of AuNPs on an Ag film with respect to a change in the gap distance $(\mathrm{nm})$. The CIE representation of the predicted colours is below and is a function of embedment depth $(0-20 \mathrm{~nm})$ shown below spectra. 84 Figure 42: Reflectance spectra of the Exp results of AuNPs on Ag film with respect to a change in the gap distance $(\mathrm{nm})$. 85

Figure 43: Reflectance spectra of the FDTD model of a 1:1 Mix on an Au film with respect to a change in the gap distance (nm).

Figure 44: Reflectance spectra of the Exp results of the 1:3 Mix on an Au film with respect to a change in the gap distance $(\mathrm{nm})$. The actual colours of the substrates are shown above the spectra (top left). The CIE representation of the colours is presented above the spectra (top right)....... 87

Figure 45: AFM image of a 1:3 mixture of Au:Ag on an Au film coated with $25 \mathrm{~nm}$ PS....... 88 Figure 46: Reflectance spectra of the FDTD model of a 1:1 Mix on an Ag film with respect to a change in the gap distance (nm).

Figure 47: Reflectance spectra of the Exp results of the 1:3 Mix on an Ag film with respect to a change in the gap distance $(\mathrm{nm})$. The CIE representation of the colours is presented above the spectra. The actual colours of the substrates are shown adjacent the spectra.......................... 90 Figure 48: AFM image of a 1:3 mixture of Au:Ag on an Ag film coated with 25 nm PS....... 91 Figure 49: $2 \mathrm{mN} / \mathrm{m}$ AuNPs on PS. AFM image (left) and the T, R, A spectra (right) ............. 104 Figure 50: $3 \mathrm{mN} / \mathrm{m}$ AuNPs on PS. AFM images (left) $10 \times 10 \mu \mathrm{m}^{2}$ (top) and $5 \times 5 \mathrm{~m}^{2}$ (bottom), and the T, R, A spectra (right)

Figure 51: T, R, A spectra for $2 \mathrm{mN} / \mathrm{m}$ (top) and $3 \mathrm{mN} / \mathrm{m}$ (bottom) AuNPs on PS before and after heating.

Figure 52: AFM images of a $1 \mathrm{mN} / \mathrm{m}$ mix on polystyrene, $10 \times 10 \mu \mathrm{m}^{2}$ (left) and $5 \times 5 \mathrm{~m}^{2}$ (right)

Figure 53: Selective Embedment on 1:1 mix at $458 \mathrm{~nm}$. In order the images represent $0 \mathrm{~s}, 5 \mathrm{~s}, 15$ s, $25 \mathrm{~s}, 35 \mathrm{~s}, 55 \mathrm{~s}, 2 \mathrm{~min}$, and $7 \mathrm{~min}$ of laser exposure. 
Figure 54: Selective Embedment on 1:1 mix at $568 \mathrm{~nm}$. In order the images represent $0 \mathrm{~s}, 5 \mathrm{~s}, 35$ s, $50 \mathrm{~s}, 65 \mathrm{~s}, 1 \mathrm{~min} 35 \mathrm{~s}, 3 \mathrm{~min}$, and $6 \mathrm{~min}$ of laser exposure 108

Figure 55: Selective Embedment on 1:1 mix at $568 \mathrm{~nm}$ then $458 \mathrm{~nm}$. Firstly, the substrate was exposed to $568 \mathrm{~nm}$ for $10 \mathrm{~s}(40 \mathrm{~mW})$, then another $10 \mathrm{~s}(50 \mathrm{~mW})$. The laser was switched to 458 and the substrate was exposed for $5 \mathrm{~s}, 10 \mathrm{~s}, 15 \mathrm{~s}, 30 \mathrm{~s}$, and $1 \mathrm{~min}$. The images are shown in chronological order.

Figure 56: Selective Embedment on 1:1 mix at $458 \mathrm{~nm}$ then $568 \mathrm{~nm}$. Firstly, the substrate was exposed to $458 \mathrm{~nm}(22 \mathrm{~mW})$ for $5 \mathrm{~s}$, then $25 \mathrm{~s}$. The laser was switched to $568 \mathrm{~nm}(34 \mathrm{~mW})$ and the substrate was exposed for $5 \mathrm{~s}, 25 \mathrm{~s}, 2 \mathrm{~min} 35 \mathrm{~s}$, and $5 \mathrm{~min}$. Finally the substrate was exposed to $568 \mathrm{~nm}(70 \mathrm{~mW})$ for $5 \mathrm{~s}$. The images are shown in chronological order.

Figure 57: Transmission spectrum of 1:3 mix on PS showing the $D_{A g}, Q_{A g}, D_{A u}$ and D-D 


\section{List of Abbreviations:}

LSP - localized surface plasmon

PSP - propagating surface plasmon

LSPR - localized surface plasmon resonance

$\mathrm{SP}$ - surface plasmon

LB - Langmuir Blodgett

$E$ - electric field

$\omega$ - excitation frequency

$\mathrm{N}$ - number density

D - electric displacement

B - magnetic induction

$\mathbf{E}$ - dielectric field

$\mathbf{H}$ - magnetic field

$\varepsilon-$ permittivity of material/dielectric function

$\mu$-permeability of material

$\varepsilon_{0}-$ permittivity of free space

$\mu_{0}$ - permeability of free space

$\mathrm{n}$ - refractive index

AuNP - gold nanoparticle

AgNC - silver nanocubes

e - elemental charge

$\mathrm{m}-$ mass

EM - electromagnetic

D - dipole

Q - quadrupole

$\mathrm{M}$ - multipole

$\mathrm{P}_{\mathrm{abs}}-$ power absorbed

$\Delta \mathrm{T}$ - temperature increase

EG - ethylene glycol

GA - glycolaldehyde

$\mathrm{T}_{\mathrm{g}}$ - glass transition temperature

ST - surface tension

FDTD - Finite Density Time Domain

CIE - Commission international de l'eclairage
UV-vis - ultra-violet-visible

I - intensity

A - absorbance

$\epsilon$ - extinction coefficient

$\mathrm{c}$ - concentration

1 - pathlength

$\mathrm{C}_{\mathrm{abs}}, \sigma_{\mathrm{abs}}-$ absorption cross-section

$\mathrm{C}_{\text {sca }}, \sigma_{\text {sca }}-$ scattering cross-section

$\mathrm{C}_{\text {ext }}, \sigma_{\text {ext }}-$ extinction cross-section

$\mathcal{N}$ - concentration of particle

$\sigma_{\text {ext }}-$ extinction of light

$\tau$ - dampening term

$\gamma$-dampening force parameter

$\mathrm{R}$ - radius

$\mathrm{c}$ - speed of light

$\lambda$ - wavelength

$Q$ - quality factor

w.r.t - with respect to

MS - metal surface

NPoM - nanoparticle on metal

$\kappa$ - thermal conductivity

$\mathrm{n}_{\mathrm{o}}$ - number of electrons

$m_{e}-$ mass of an electron

$\mathrm{T}$ - metal film thickness

$\mathrm{k}$ - incoming wavevector

$J_{0}$ - joule parameter

$\mathrm{V}$ - volume

$\beta$ - shape correcting coefficient

SADC - sodium acetone dicarboxylate

LS - Langmuir Schaffer

$\mathrm{F}$ - vertical force

$\mathrm{P}_{\text {surf }}$ - surface pressure

PVP - poly(vinyl) pyrrolidone 


\section{List of Appendices:}

Figure 49: $2 \mathrm{mN} / \mathrm{m}$ AuNPs on PS. AFM image (left) and the T, R, A spectra (right) 104

Figure 50: $3 \mathrm{mN} / \mathrm{m}$ AuNPs on PS. AFM images (left) $10 \times 10 \mu \mathrm{m}^{2}$ (top) and $5 \times 5 \mu \mathrm{m}^{2}$ (bottom), and the T, R, A spectra (right) 105

Figure 51: T, R, A spectra for $2 \mathrm{mN} / \mathrm{m}$ (top) and $3 \mathrm{mN} / \mathrm{m}$ (bottom) AuNPs on PS before and after heating. 106

Figure 52: AFM images of a $1 \mathrm{mN} / \mathrm{m}$ mix on polystyrene, $10 \times 10 \mu \mathrm{m}^{2}$ (left) and $5 \times 5 \mathrm{~m}^{2}$ (right)

Figure 53: Selective Embedment on 1:1 mix at $458 \mathrm{~nm}$. In order the images represent $0 \mathrm{~s}, 5 \mathrm{~s}, 15$ s, $25 \mathrm{~s}, 35 \mathrm{~s}, 55 \mathrm{~s}, 2 \mathrm{~min}$, and $7 \mathrm{~min}$ of laser exposure.

Figure 54: Selective Embedment on 1:1 mix at $568 \mathrm{~nm}$. In order the images represent $0 \mathrm{~s}, 5 \mathrm{~s}, 35$ s, $50 \mathrm{~s}, 65 \mathrm{~s}, 1 \mathrm{~min} 35 \mathrm{~s}, 3 \mathrm{~min}$, and $6 \mathrm{~min}$ of laser exposure. 108

Figure 55: Selective Embedment on 1:1 mix at $568 \mathrm{~nm}$ then $458 \mathrm{~nm}$. Firstly, the substrate was exposed to $568 \mathrm{~nm}$ for $10 \mathrm{~s}(40 \mathrm{~mW})$, then another $10 \mathrm{~s}(50 \mathrm{~mW})$. The laser was switched to 458 and the substrate was exposed for $5 \mathrm{~s}, 10 \mathrm{~s}, 15 \mathrm{~s}, 30 \mathrm{~s}$, and $1 \mathrm{~min}$. The images are shown in chronological order. 109

Figure 56: Selective Embedment on 1:1 mix at $458 \mathrm{~nm}$ then $568 \mathrm{~nm}$. Firstly, the substrate was exposed to $458 \mathrm{~nm}(22 \mathrm{~mW})$ for $5 \mathrm{~s}$, then $25 \mathrm{~s}$. The laser was switched to $568 \mathrm{~nm}(34 \mathrm{~mW})$ and the substrate was exposed for $5 \mathrm{~s}, 25 \mathrm{~s}, 2 \mathrm{~min} 35 \mathrm{~s}$, and $5 \mathrm{~min}$. Finally the substrate was exposed to $568 \mathrm{~nm}(70 \mathrm{~mW})$ for $5 \mathrm{~s}$. The images are shown in chronological order. 110 Figure 57: Transmission spectrum of 1:3 mix on PS showing the $D_{A g}, Q_{A g}, D_{A u}$ and D-D plasmon modes. The D-D resonance transmits lower than $\mathrm{D}_{\mathrm{Au}}$. 


\section{Introduction:}

\subsection{Motivation and outline}

Metal nanoparticles, silver (Ag) and gold ( $\mathrm{Au}$ ), have been extensively explored in the field of nanoscience owing to their strong plasmonic responses in the visible region of the electromagnetic spectrum. ${ }^{1-3}$ In particular, these nanoparticles (NPs) exhibit a fascinating optical property known as localized surface plasmon resonance (LSPR). These optical properties have been of interest for centuries, and scientific research dates as far back as recorded by Faraday in his synthesis of colloidal Au. ${ }^{4}$ Later, Mie would provide the classical physical model describing the extinction (absorption + scattering) spectrum of spherical particles of arbitrary size by providing specific solutions to Maxwell's equations., 5 Though, it is noteworthy to add that, historically, a most prominently employed use of metal nanoparticles has been in stained glass and ceramic pottery, as observed in the Lycurgus cup (Byzantine empire, 4th century A.D.), producing marvellously coloured materials - even before their scientific understanding. ${ }^{7,8}$ Many new advances have been made in nanoscience taking advantage of the NP optical properties for applications in sub-wavelength optical devices, ${ }^{8}$ biosensing, ${ }^{9}$ photodetection, ${ }^{10}$ surface enhanced sensing and spectroscopy, ${ }^{11}$ and many others. As observed, a result of the LSPR is the powerful colour that is obtained upon interaction with light. This effect has been manipulated by imparting specific changes to the local environment of the nanomaterial such as changing the shape, ${ }^{12}$ size, ${ }^{13}$ aspect ratio, ${ }^{14}$ or bringing the object near a dielectric substrate ${ }^{15}$ to name a few. Although, there has not been any research on the optical properties of mixtures of individual nanoparticles of different composition, in particular mixtures of $\mathrm{Au}$ and $\mathrm{Ag}$ nanoparticles.

This research analyzes the optical properties of nanomaterials, focusing on mixtures of nanoparticles of different composition. There is some existing research that specifies the ability to 
modify the current scale of the plasmonic colouring regime and better manipulate the interaction of light and material by using mixtures of nanoparticles of the same composition, but with varying sizes or shape. ${ }^{13}$ This led to the design of the mixtures of the NPs of different type. The optical properties of silver (namely AgNCs) both in solution, and when supported atop a substrate, such as polystyrene (PS), have been well-explored by my cohorts and predecessors in the Ianoul group. ${ }^{16-18}$ For gold, we were not as well adept in terms of supporting the material on substrates, but we had the means for further exploration. Using Langmuir-Blodgett (LB) as the principal technique, monolayers of NPs were prepared for deposition onto thin films of polymers. It was predicted that the mixtures of nanoparticles would lead to one simple system containing multiple plasmonic modes across the visible spectrum.

Firstly, the nanoparticles were to be deposited with only particles of the same composition (i.e., only AgNCs, and only AuNPs). The information obtained from these depositions informed us on the optical properties exhibited by the nanomaterials containing particles of a single type. As such, once said optical properties were understood, simple computations could be proceeded for the design of mixtures of nanoparticles of different composition and ratios. The mixture of nanoparticles can then be deposited onto substrates using LB methods. On top of a substrate, the nanoparticles exhibit optical properties of variance from their in-solution counterparts. As the substrate is heated above the polymer's glass-transition temperature, the nanoparticles begin to embed, and the changes to their dielectric environment result in changes to their optical response.

I also predict the ability to control the nanoparticle embedment via spectrally selective thermoplasmonic embedment. Using a laser set at specific wavelengths, which are in resonance with the dipolar LSPR, the nanoparticles will selectively embed into the polymer film. The new nanoarchitecture containing mixtures of $\mathrm{Au}$ and $\mathrm{Ag}$ nanoparticles, would have distinct plasmonic 
modes for either particle. So, it should be possible to embed either individual AgNCs or AuNPs using selective thermoplasmonic embedment at their respective LSPR.

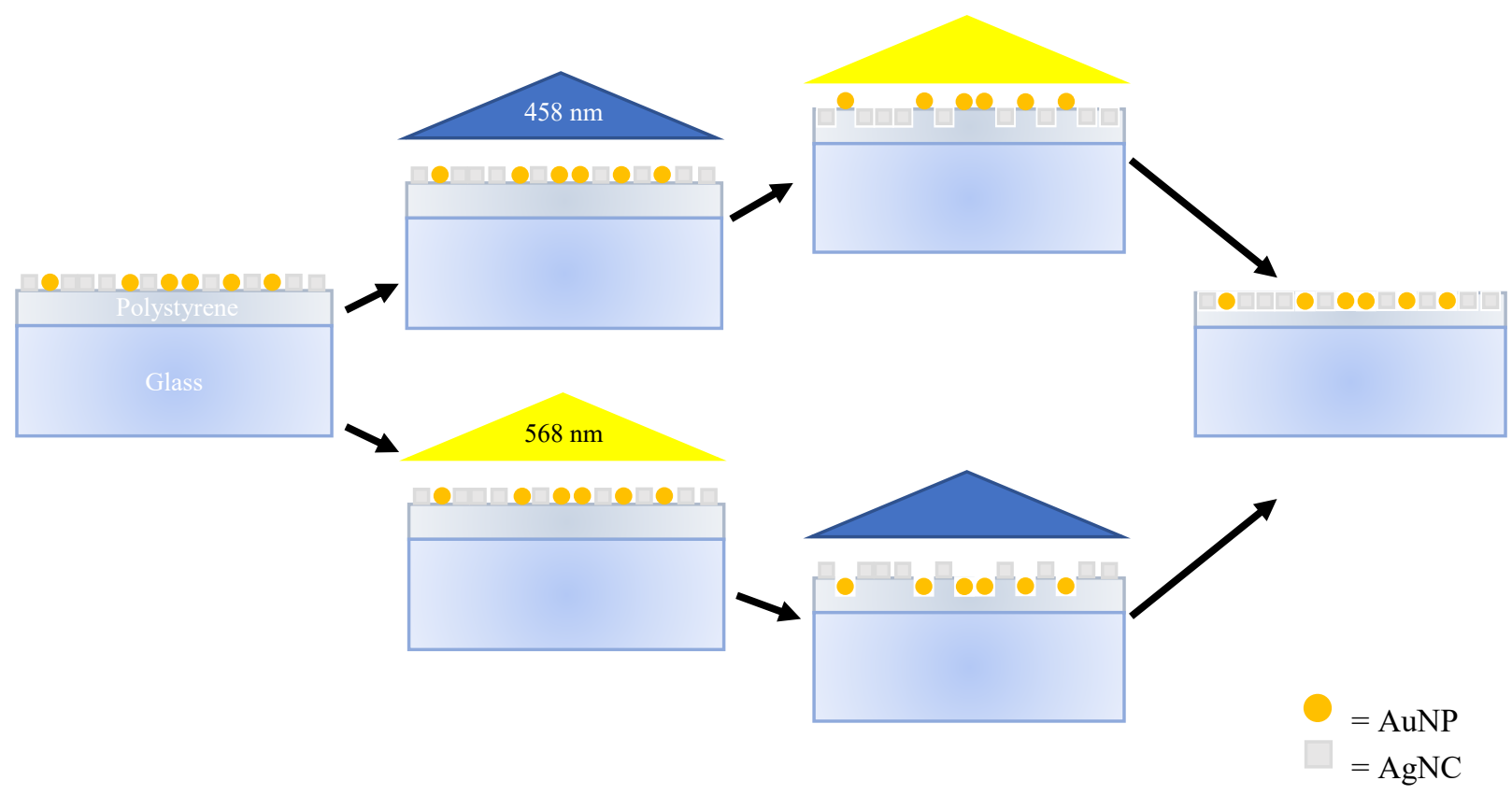

Figure 1: Schematic of the selective thermoplasmonic embedment of nanoparticles upon laser irradiance at $458 \mathrm{~nm}$, and $568 \mathrm{~nm}$.

Once the new nanoarchitecture is understood, the mixture can be deposited onto ultra-thin $(<30 \mathrm{~nm})$ polymer films which have been coated onto nano-scale metal films. Doing this allows for plasmon-plasmon coupling through a dielectric gap, allowing us to take advantage of plasmonic gap-modes to produce differently coloured materials. Thus, the exploration of new and interesting materials which expand the plasmonic colouring regime via plasmonic gap-modes is permitted.

\subsection{Plasmonics}

Plasmonics is considered a subfield of photonics concerned with the manipulation of light at the nanoscale. ${ }^{19}$ A plasmon is defined as a quantum of plasma oscillation (n.b., quantum is the smallest unit of discrete energy). Electromagnetic waves (light) can be manipulated, localized and 
guided below the diffraction limit down to nanometer-scale lengths, and these effects are associated to plasmonics. ${ }^{20}$ Metals such as $\mathrm{Ag}$ and Au are able to support surface plasmon polariton modes, making them key functional components of plasmonics. Surface plasmon polaritons are electromagnetic waves that are coupled with the collective oscillation of free electrons in a metal. ${ }^{20}$ Although they have a large associated variety, plasmonic nanostructures may be differentiated by the plasmonic modes that they exhibit; the plasmon modes, being localized surface plasmons (LSPs) and propagating surface plasmons (PSPs). ${ }^{20,21}$

For a given LSP, there is a time-varying electric field $\left(E_{o}\right)$ associated with the light which exerts a force on the conduction band of a metal, causing collective oscillation (fig. 1 a) ${ }^{20}$ The oscillation is in resonance with incident light at a specific excitation frequency $(\omega)$, resulting in the strong oscillation of surface electrons, hence, the phenomenon of localized surface plasmon resonance (LSPR). ${ }^{20} \mathrm{~A}$ material containing a LSPR has an electric field that is uniform when excited by light as the dimensions of the material are less than the wavelength of light. ${ }^{20}$ The

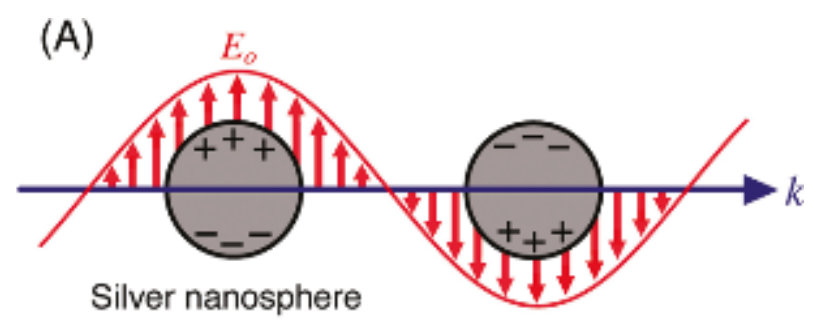

(B)

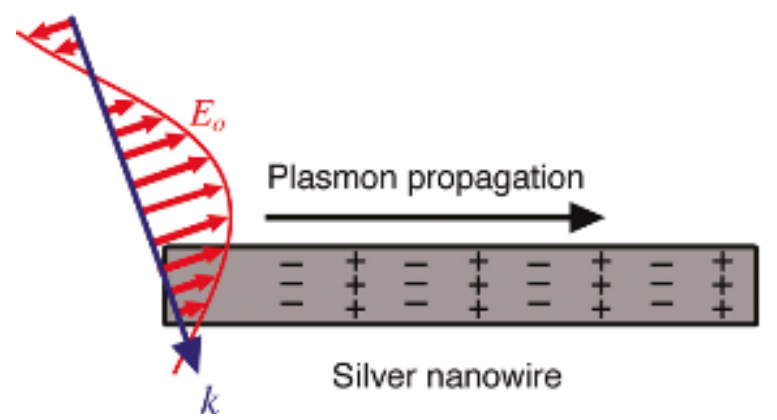

electromagnetic field close to the surface of the particle is enhanced by the LSPR from charge separation by the collective oscillation. ${ }^{20}$ For a given nanostructure, there exists a maximum absorption at the SPR frequency. ${ }^{22}$

In the case of PSPs, for structures such as nanowires or thin metal films, the SPR will propagate along the dimension which is still exhibiting bulk effects (i.e. larger than the wavelength of incident light). ${ }^{20}$ In this case, $E_{o}$ 2011 American Chemical Society 
is not uniform across the structure. Nanoparticles having larger sizes, different morphologies and different dielectric surroundings can support different types of SPR. ${ }^{22}$

\subsubsection{Light Interaction: Simple Plasmonic Models}

Provided the simple model for a metal surrounded by a gas of free electrons moving against a fixed background of positive carriers, the representation of a bulk plasmon can be achieved. The number density $(N)$ of immobilized positive ions is constant in space and time, hence, at equilibrium the free electron number density is also $N .^{23}$ If the free electrons are disturbed from equilibrium, the non-uniform charge distribution will enact an electric field (E) which tends to restore the charge neutrality. ${ }^{23}$ The electrons can acquire enough momentum from the E-field to overshoot the equilibrium, resulting in an oscillation. ${ }^{23}$ This collective oscillation is known as the plasma oscillation, and its bulk plasma frequency $\left(\omega_{p}\right)$ can be given by:

$$
\omega_{p}=\sqrt{\frac{N e^{2}}{m \varepsilon_{0}}}
$$

where $\mathrm{e}$ is the elemental charge, $\mathrm{m}$ is the mass, and $\varepsilon_{0}$ is the permittivity of free space.

Nanoparticles under the effect of an electromagnetic (EM) field have electrons which oscillate, transforming that energy from the incident EM wave in another form of energy. With enough power from the light source, these particles are known to undergo radiative dampening, ${ }^{24}$ thermal dissipation, ${ }^{25}$ or even hot carrier (electron) generation for use in catalytic processes. ${ }^{26}$ NPs can take on many shapes or sizes, and by using the cross-sectional approach to model the interaction of light with matter, the geometric shape of the particle is factored out. The optical cross-section for a particle is the representation of how much energy is lost to absorption or scattering processes. ${ }^{15}$ The sum of the absorption $\left(\mathrm{C}_{\mathrm{abs}}\right)$ and scattering $\left(\mathrm{C}_{\mathrm{sca}}\right)$ cross-sections of a wave propagating toward a sinqgle particle, multiplied by the total concentration of particles $(\mathcal{N})$ is the extinction of light $\left(\sigma_{\text {ext }}\right){ }^{15}$ 


$$
\sigma_{\text {ext }}=\mathcal{N}\left(C_{a b s}+C_{s c a}\right)
$$

A materials extinction can be represented by its dielectric function $(\varepsilon)$, as $\varepsilon$ is a representation of how the material interacts with light. The dielectric function for an LSPR sources local environment is complex; the dielectric function contains a real and imaginary part $\varepsilon_{r}$ (the slowing of the propagation of light) and $\varepsilon_{i}$ (loss of energy by absorption or scattering) respectively (eqn. 3). ${ }^{27}$ However, contained within the dielectric function is the real and imaginary parts of the refractive index (n) of the local environment (eqn. 4 and 5). From this, it is concluded that the LSPR frequency is dependent on the refractive index of the local environment. Given a small, positive imaginary component and a largely negative real part, strong plasmonic responses in absorption, scattering and extinction can be observed (fig. 1.3). ${ }^{27,28}$

$$
\begin{gathered}
\varepsilon=\varepsilon_{r}+i \varepsilon_{i} \\
\varepsilon_{r}=n_{r}{ }^{2}+n_{i}{ }^{2} \\
\varepsilon_{i}=2 n_{r} n_{i}
\end{gathered}
$$

Experimental measurements for the dielectric functions $\left(\varepsilon_{\text {exp }}(\omega)\right)$ of bulk metals have revealed that there are contributions from interband $\left(\varepsilon_{\text {inter }}(\omega)\right)$ and intraband $\left(\varepsilon_{\text {intra }}(\omega)\right)$ electron transitions contained within, and they are presumed to be additive. ${ }^{15}$

$$
\varepsilon_{\text {exp }}(\omega)=\varepsilon_{\text {inter }}(\omega)+\varepsilon_{\text {intra }}(\omega)
$$

Interband transitions are a result of electron transitions from occupied to unoccupied bulk bands separated by an energy gap. ${ }^{15}$ Intraband transitions come from electron transitions at the Fermilevel in incompletely filled bands or when a filled bands energy overlaps that of an empty band. Excited interband electrons relax from restoring forces, defined by the energy difference between the excited and ground states (this is thought of as electron-hole pair recombination). Intraband transitions are small, forbidden transitions at the Fermi-level typically generated by small photon 
energies and allowed by phonon vibrations. ${ }^{15}$ These electrons are essentially "free" electrons and their contributions to $\varepsilon_{\exp }(\omega)$ can be represented by the Drude model:

$$
\varepsilon_{r}(\omega)=1-\frac{\omega_{p}{ }^{2}}{\omega\left(\omega+\frac{i}{\tau}\right)} \Rightarrow 1-\frac{\omega_{p}{ }^{2}}{\omega^{2}+\gamma^{2}}
$$

here, $\omega_{p}$ is the bulk plasma frequency and $1 / \tau$ is the damping constant due to the dispersion of electrons. For a random electron, the mean free path at room temperature is typically a few nanometers (comparable to the particle size), hence, the dampening term $(\tau)$ needs to be introduced to account for the surface scattering of electrons. The dampening term is a representation of characteristic changes in the mean free path of the free electrons (e.g., NP size and shape). ${ }^{29} \mathrm{~A}$ simplification is made by introducing a new term, $\gamma$, the dampening force parameter $(\omega i / \tau),{ }^{29}$ and eqn. 6 can be rearranged to calculate for the bulk plasmon frequency (eqn. 8).

$$
\omega_{p}=\sqrt{\left(1-\varepsilon_{r}\right)\left(\omega^{2}+\gamma^{2}\right)}
$$
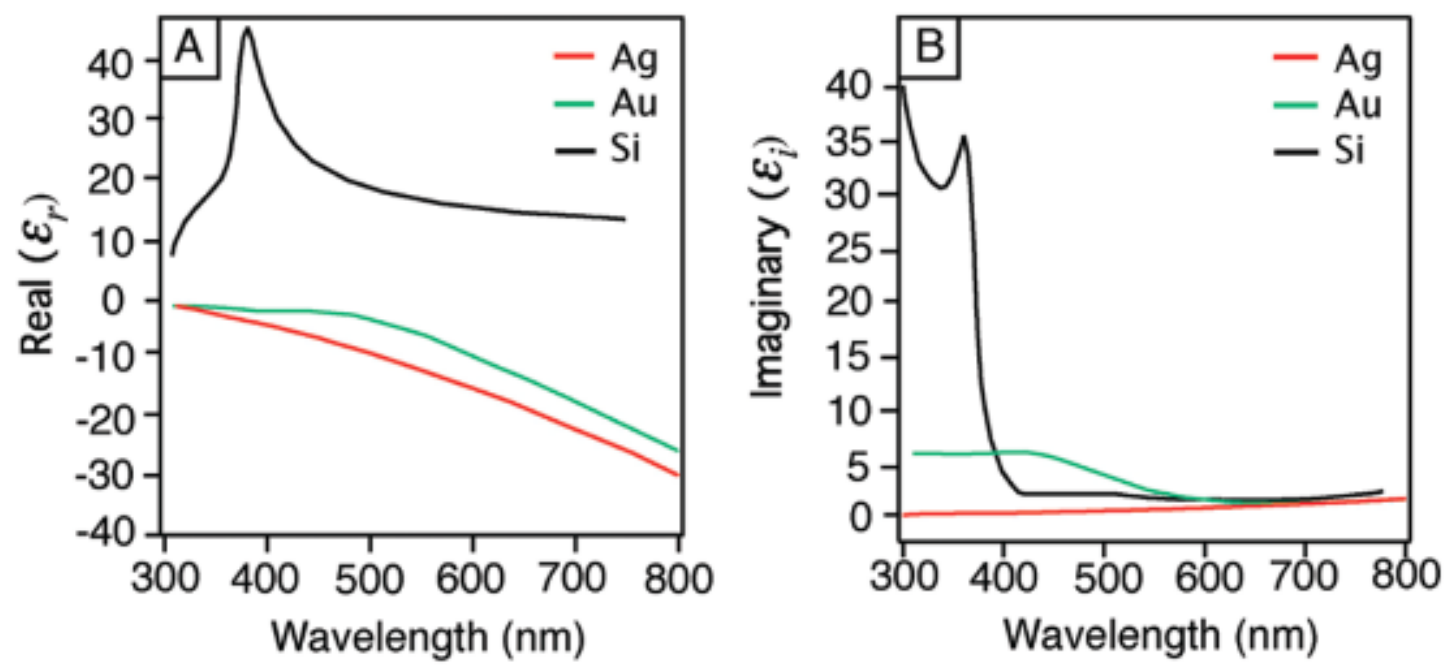

Figure 3: Plots of the real (A) and imaginary (B) components of the dielectric function of Ag, $\mathrm{Au}$ and $\mathrm{Si}$ as a function of wavelength. Reproduced with permission from ref 20. Copyright 2011 ACS.

By employing Mie Theory based calculations to solve Maxwell's equations, LSPR phenomena can be described for the extinction cross-section of a metal nano-sphere: ${ }^{20,27}$ 


$$
C_{e x t}=\frac{24 \pi^{2} R^{3} \varepsilon_{m}^{\frac{3}{2}}}{\lambda}\left[\frac{\varepsilon_{i}}{\left(\varepsilon_{r}+2 \varepsilon_{m}\right)^{2}+\varepsilon_{i}^{2}}\right]
$$

where $C_{\text {ext }}$ is the extinction cross section, $R$ is the radius, and $\varepsilon_{m}$ is the relative dielectric constant of the medium surrounding the nanosphere. This equation shows that the interaction between a metal nanoparticle and light depends strongly on its dielectric properties ( $\varepsilon_{r}$ (real) and $\varepsilon_{i}$ (imaginary)). As the denominator of the dielectric portion (bracketed) approaches zero, $\mathrm{C}_{\mathrm{ext}}$ becomes very large and absorption and scattering strength increases. This is the resonance condition indicating that when $\varepsilon_{r}$ is close to $-2 \varepsilon_{m}$ we approach infinite resonance, which is impossible for our typical dielectrics (having $\varepsilon_{r}$ between 1 and 50). ${ }^{20}$ Generally, no LSP or PSP sufficiently strong enough for use in plasmonics can be generated without a negative $\varepsilon_{r}$. It can also be concluded that if the $\varepsilon_{i}$ value is large (fig. 3), the plasmon will be weaker. Incident radiation is typically in the visible-near IR range $(\gamma \ll \omega)$, and from Mie theory the resonance condition is $\varepsilon_{r}=-2 \varepsilon_{m}$ allowing for the simplification and solution of eqn. 8 with respect to the maximum resonant excitation frequency $\left(\omega_{\max }\right){ }^{30}$

$$
\omega_{\max }=\frac{\omega_{p}}{\sqrt{\left(2 \varepsilon_{m}+1\right)}}
$$

This equation can be converted to calculate for wavelength $(\lambda)$ rather than frequency using the following expression, $\lambda=\frac{2 \pi c}{\omega}$, and with respect to RI $\left(\varepsilon_{m}=n_{m}{ }^{2}\right)$ to give: ${ }^{27}$

$$
\lambda_{\max }=\lambda_{p} \sqrt{2 n_{m}^{2}+1} \cong \lambda_{p} \sqrt{2} n_{m}
$$

where $\lambda_{\max }$ is the LSPR peak maxima, $\lambda_{p}$ is the wavelength of the bulk plasma material and $n_{m}$ is the refractive index of the medium. This equation confirms that as the local refractive index of the material changes, there will be corresponding changes to the plasmon peak position (i.e., increasing the local refractive index will result in a correspondent red shift in the spectrum). 


\subsubsection{Choice of Metal Nanostructures}

Plasmons have high sensitivity to changes in their local electric field; as the shape or permittivity of a material changes, the plasmonic frequency can be tuned across a wide range of wavelengths. ${ }^{20,29,31}$ Metals are used for exploration of the optical properties of SPR supporting materials with the primary focus being on coinage metals ( $\mathrm{Au}, \mathrm{Ag}$ and $\mathrm{Cu}),{ }^{19}$ although other metals like $\mathrm{Pt},{ }^{32}$ and $\mathrm{Al},{ }^{33}$ have also been explored for their plasmonic properties. Research examines thoroughly the robust materials $\mathrm{Ag}$ and $\mathrm{Au}$ as choice elements in plasmonics. These metals bring forth their own advantages and disadvantages. Gold is inert, thus very hard to oxidize and hence biocompatible, providing interdisciplinary usability. ${ }^{34-37}$ Though, it should also be noted that $\mathrm{Au}$ is a very expensive metal. ${ }^{37}$ Contrarily, silver has sharp resonances and is able to support higher order plasmon modes, however it is very susceptible to oxidation and is quite biologically active..$^{20,38}$

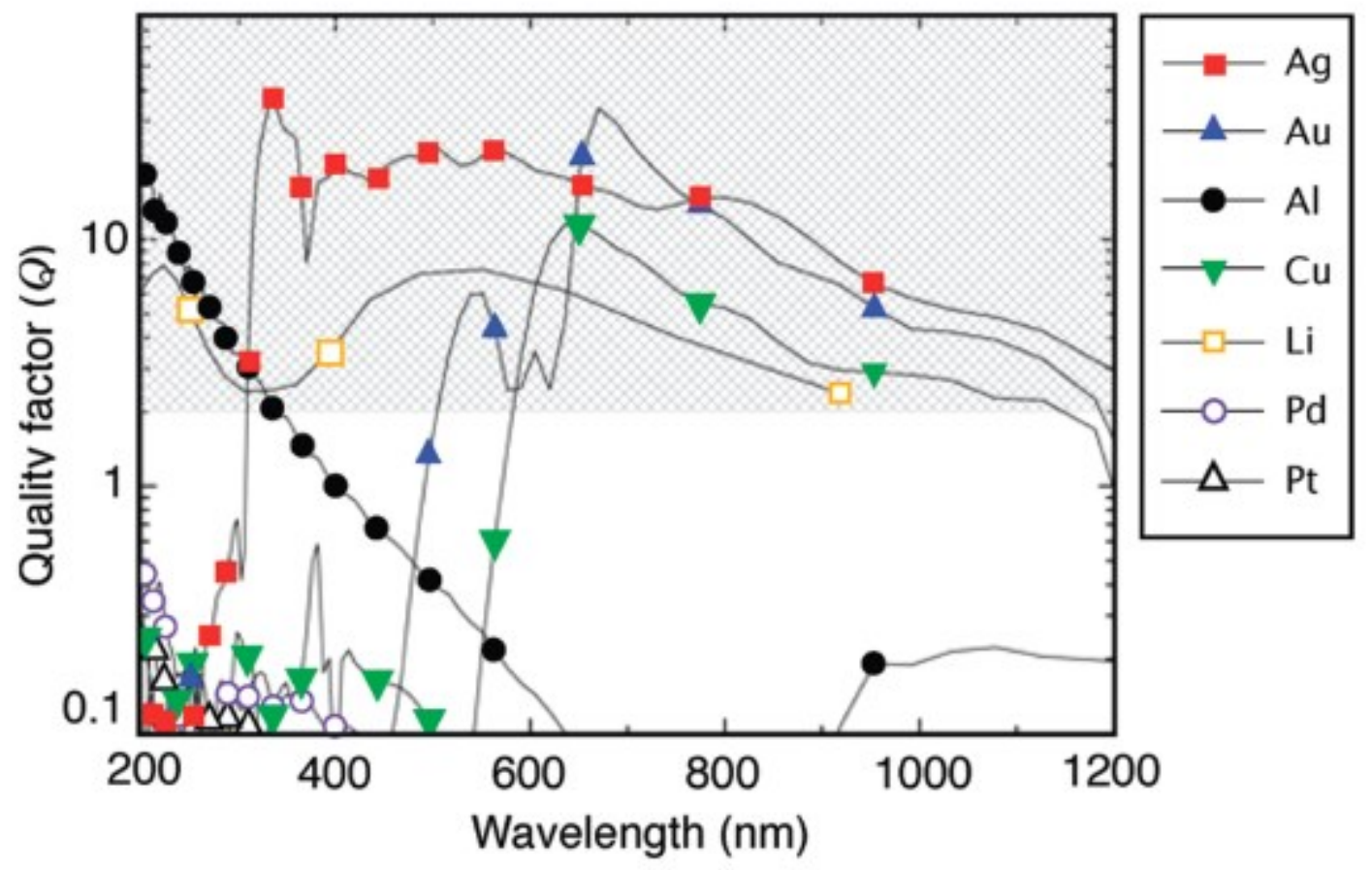

Figure 4: Quality factor (Q) of the LSPR of different metals at the metal/air interface. The shaded area indicates the region at which $\mathrm{Q}$, and hence plasmonic response is strongest. Reproduced with permission from ref 20. Copyright 2011 ACS 
Strength of the SP being supported by a material is directly related to the real and imaginary functions of the material and can be measured using the quality factor (Q), ${ }^{36}$

$$
Q=\frac{\omega\left(\frac{d \varepsilon_{r}}{d \omega}\right)}{2\left(\varepsilon_{i}\right)^{2}} \approx-\frac{\varepsilon_{r}}{\varepsilon_{i}}
$$

SP strength is directly correlated to quality factor (i.e. larger Q means stronger SPs and SPRs), ideally obtaining a Q larger than $\sim 10$ for a majority of plasmonic applications. ${ }^{20}$ The quality factor is a numerical representation of how sharp/narrow a plasmon is at a given wavelength and leads to strong local field enhancement and higher refractive index sensitivity. ${ }^{37}$ Materials with higher Q have stronger local-field enhancement. Fig. 4 shows clearly that silver has the best quality factor across the UV-Vis-NIR range with Au being the next best metal. ${ }^{20}$

Another method to quantify plasmonic materials is through the refractive index sensitivity $\left(S=\frac{d \lambda_{p}}{d n} ; \mathrm{nm} / \mathrm{RUI}\right)$; changes in the in the refractive index of the local environment results in changes to total SPR. An excellent report for these values shows that, AgNCs had an experimental refractive index sensitivity of $336 \pm 15 \mathrm{~nm} / \mathrm{RIU}$ at $534 \mathrm{~nm}$, while gold nanocubes had a refractive index sensitivity of $147 \pm 3 \mathrm{~nm} / \mathrm{RIU}$ at $563 \mathrm{~nm} .{ }^{39}$ The refractive index sensitivities indicate that Ag will experience more noticeable changes than $\mathrm{Au}$ with changes to the dielectric environment (e.g., bringing the particle closer to a surface or embedding the particle into a polymer). The broad and high nature of silvers quality factor makes it highly desired for use in sensors. ${ }^{40}$

\subsubsection{Shape and Size Effects on Plasmonic Materials}

As a NPs size increases, the electron cloud experiences more delocalization and depolarization, dampening the electron oscillations to lower energies; ${ }^{41}$ as the NP size increases there is a red-shift in the LSPR. ${ }^{42}$ Small spherical particles are the simplest geometric model in plasmonics. For a small spherical NP, there is a dipolar (D) distribution of charge at the surface. ${ }^{41,42}$ Increasing the size of the NP results in the appearance multipolar charge distributions of higher 
order resonant modes. ${ }^{42}$ These higher order plasmon modes exhibit higher angular momenta, and hence, are of higher energy. ${ }^{5}$

Shape also plays a crucial role in plasmonics, affecting the overall extinction of the NPs. For example, NPs containing edges and vertices can facilitate multipolar resonances needed to support higher order resonate modes such as the quadrupolar $(\mathrm{Q})$ or multipolar $(\mathrm{M})$ modes (even in small NPs). ${ }^{43}$ Advantageously, spectrophotometry can be used to determine the sharpness of edges due to rounded edges having broad, higher energy resonances resulting from less delocalization of the electron clouds (smaller restoring force). ${ }^{15}$ For cubic NPs, charge accumulation at vertices and edges causes an extremely high electric field intensity, thus, NPs containing many edges and vertices have higher sensitivity to their local environment. ${ }^{27}$

\subsubsection{Coupling of Plasmons}

Plasmonic materials brought into proximity of each other have potential for their plasmonic modes to couple together, generally resulting in a new red-shifted plasmonic mode. The oscillating dipoles of the particles can interact depending on the polarization of incident light with respect to the particles, forming parallel or perpendicular coupling modes. Oscillations parallel to the incident radiation interact with the light, exemplifying the "bright mode". ${ }^{4}$ Perpendicular oscillations are considered "dark mode" interactions forming no net electronic dipole and is not observed in the extinction spectra. ${ }^{44}$

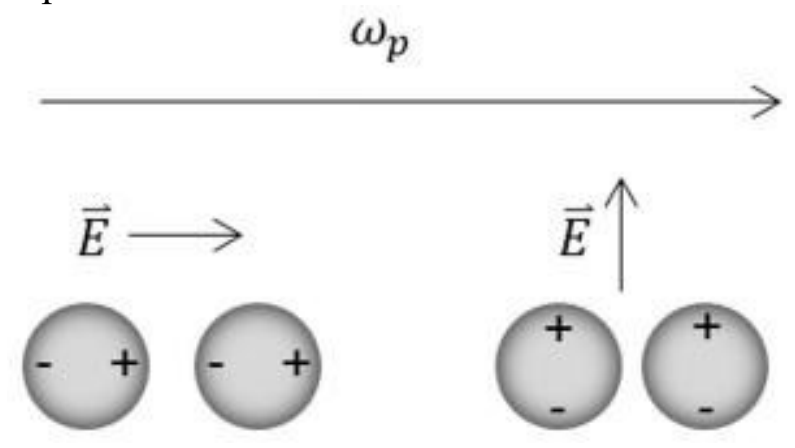

Figure 5:Illustration of parallel and perpendicular coupling modes of a NP dimer 
In the coupling regime, the bright mode is spectrally red shifted, and the dark mode is slightly blue shifted from their individual counterparts.

Frequently, plasmon coupling is modelled using hybridization arguments analogous to what is seen in molecular or hybrid orbital theory (fig. б). In this model, plasmons undergo electromagnetic mixing to form new hybrid modes. These modes split into a low energy "bonding" mode and higher energy "anti-bonding" mode. ${ }^{44}$ The planar light interacts strongly to the bright, bonding mode, however, the dark anti-bonding mode does not gain enhancement from the planar incident light; the dark mode gains no dipolar enhancement. The bright mode experiences a sharp resonance with the incident light resulting in a strong LSPR which has been red shifted (lower energy) w.r.t. the original LSPR of the individual NPs. As the interaction between the two particles increases, the energy difference in the two hybrid modes increases, assuming that there is no quantum tunnelling. ${ }^{44}$ It is of importance to note that the splitting is asymmetric; the anti-bonding mode undergoes a slight blue shift (higher energy), compared to a larger red shift in the bonding. Although not always desired, an interesting phenomenon of the hybridization model for a dimer is the ability to access higher energy angular momenta within the NP system via coupled plasmonic materials. $^{45}$

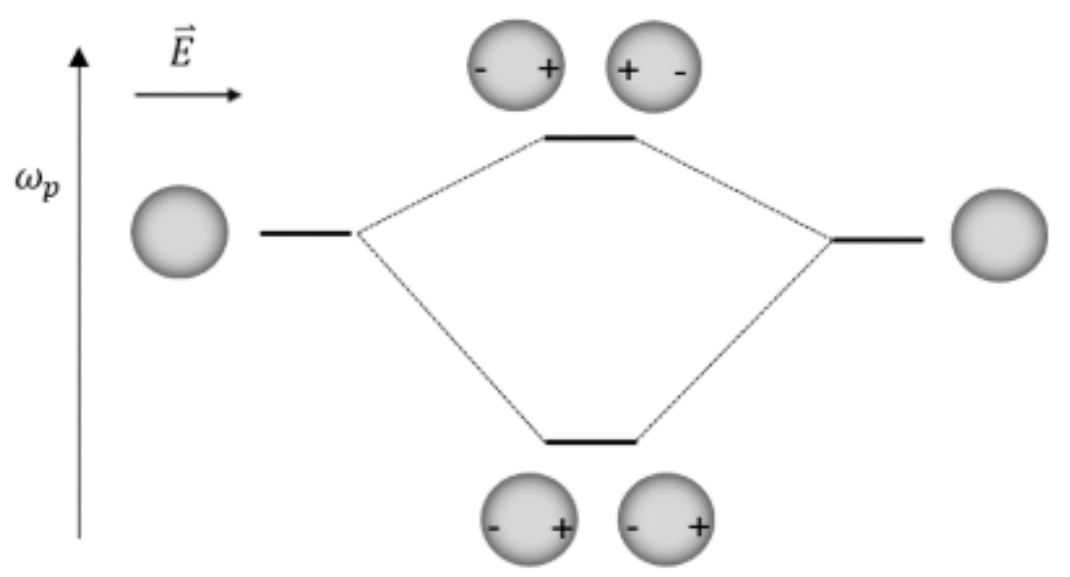

Figure 6: Schematic of NP dipolar coupling using the hybridization model to give the low energy bonding mode and higher energy anti-bonding mode 
As the distance between the particles is decreased, the interaction energy of plasmons is increased. It has been shown that the energy increases exponentially with decreasing distance; at edge-edge distances of $3.0 \mathrm{~nm}$ the peak shift is zero, but as the gap distance approaches 0 there is an exponential increase in peak shift to $0.10 \mathrm{~nm} .{ }^{45}$ This suggests that there would be an everincreasing red shift in the bonding dipolar plasmon as the particles approach each other, and until they are in direct contact. ${ }^{46,47}$ If conductive overlap is achieved, a new charge transfer plasmon appears where conduction electrons can flow back and forth between the NPs presenting a new, unique plasmon mode. ${ }^{48}$ This new mode replaces the bonding dipolar mode, originally appearing at this peak position, then blue shifts with increasing conductive overlap. ${ }^{48}$ It is also possible for other charge transfer modes to appear. ${ }^{49}$

The classical description becomes flawed at NP spacing below $1 \mathrm{~nm}$, failing to coincide with experimental observations. ${ }^{50}$ Within this regime, it can become possible for electrons to tunnel between adjacent nanoparticles. Moreover, finite electron density in the interparticle cavity may mask plasmonic interactions responsible for the strong redshift observed in coupled NPs. ${ }^{50}$ Due to these effects, the bonding dipolar plasmon does not redshift to the degree predicted by classical theory, and the onset of the charge transfer plasmon occurs before conductive contact can be made, at gap distances of about $0.5 \mathrm{~nm}$ or less. ${ }^{50,51}$

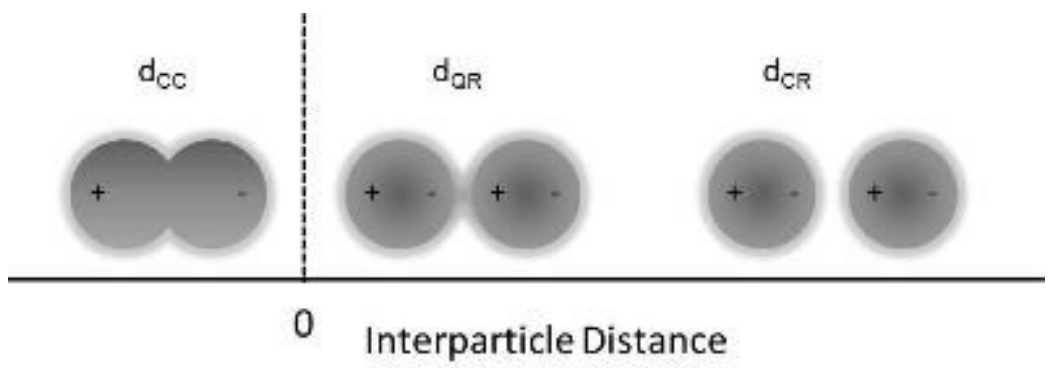

Figure 7: Schematic illustration of the classical regime $\left(\mathrm{d}_{\mathrm{CR}}\right)$, quantum regime $\left(\mathrm{d}_{\mathrm{QR}}\right)$, and conductive contact regime $\left(\mathrm{d}_{\mathrm{CC}}\right)$ 
There exist three distinct regimes for nanoparticles in proximity of each other, Figure 7; the classical or non-contact regime, the quantum tunneling regime, and the conductive contact regime. ${ }^{50}$ The distances at which these regimes occur depend on the plasmonic material, size, geometry and dielectric environment. ${ }^{50,52}$ However, measurements for small Ag- and AuNPs can aid in the understanding of the system. At distances larger than $5 \AA$, electron transfer is not significant, hence, this system can be modeled with classical electrodynamics. ${ }^{51,52}$ The bonding dipolar mode redshifts as an individual unit, and the localized electric field enhancement increases with reduced gap distance. ${ }^{51,52}$ Below $5 \AA$, the tunneling probability of electrons begins to become significant; the bonding dipolar mode no longer shifts singularly as the particles are brought closer together. At distances less than $\sim 2 \AA$ the potential barrier drops below the Fermi level allowing electrons to freely flow between nanoparticles. ${ }^{48}$ As such, electron density can now exist in the junction, and the bonding plasmon is replaced with charge transfer plasmons which blue-shift with decreasing interparticle distance. This constitutes the charge transfer regime. Charge transfer plasmons are plasmonic modes that span both particles but behave as if they are a single, elongated non-spherical particle. ${ }^{52}$ The high charge densities on opposite sides of the junction are neutralized by the onset of electron transfer, resulting in drastic decreases in field enhancement. ${ }^{51}$

\subsubsection{Plasmon-Substrate Interactions}

It is known that nanoparticles have high sensitivity to their local environment. NPs supported atop a dielectric substrate undergo a breaking of the symmetry of their dielectric environment making it possible for large changes in the plasmon modes present. ${ }^{53}$ When this happens, the plasmon induced charge density of the nanoparticles is masked by induced surface charges at the substrate, resulting in a superimposed electromagnetic image of the NPs E-field on the substrate. ${ }^{54}$ 

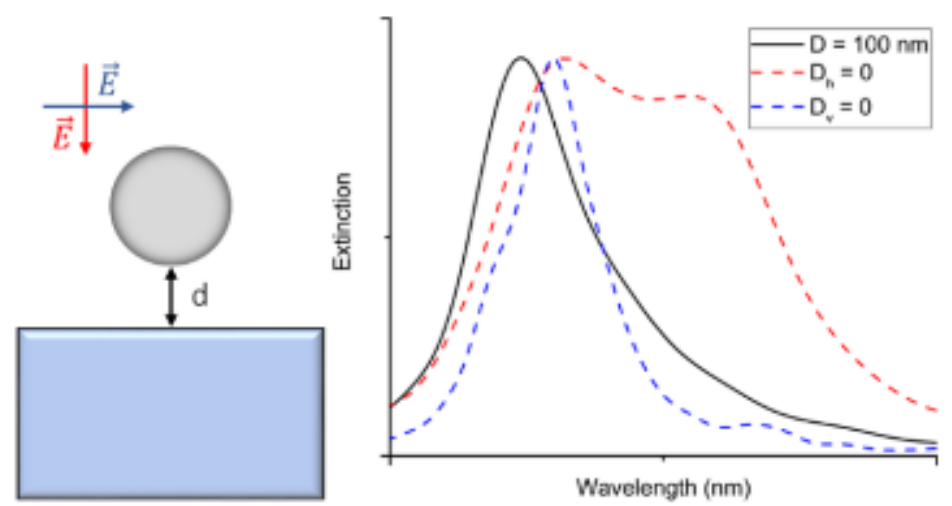

Figure 8: Illustration (left) of substrate distance dependence of spherical NP coupling, FDTD simulated extinction spectra of a nanoparticle $100 \mathrm{~nm}$ above the dielectric substrate (Solid line), and on the substrate (Dashed line), with normal (to the substrate) (Dh, Red) and parallel (Dv, Blue) polarized light.

The interaction of the plasmonic material with a dielectric substrate is very distance dependent and can present higher order plasmon modes as the symmetry is broken by the substrate (as shown by $\mathrm{D}_{\mathrm{h}}$, fig. 8). ${ }^{55}$ Charge separation of the dielectric results in a disruption of symmetry of the plasmonic particle. The degeneracy of the plasmon modes is broken by this asymmetry; ${ }^{55}$ plasmon modes having charge densities closer to the surface will couple more than modes that are further away from the substrate. Bringing the particle closer to the substrate enhances the interaction with parallel light $\left(D_{h}\right.$ mode $)$ which is much stronger than the perpendicular $\left(D_{v}\right)$ light, hence $D_{h}$ is more red shifted. Hybridization of plasmon modes is enabled by the symmetry breaking by the dielectric substate due to the formation of multipoles. ${ }^{55}$

From a spherical NP a dipolar distribution of charges is typically observed, while nonspherical NPs have LSPRs with uneven charge distributions resulting in unique absorption and scattering processes. Sharp featured NPs (featuring edges and vertices) have that distinctive uneven charge distribution leading to enhancement of the electric field around these sharp features and subsequently their ability to support higher order resonant modes (even within small particles). ${ }^{42}$ As well, it has already be shown that nanomaterials with structural anisotropy (e.g., 
nanowires) have the ability to support different longitudal and transverse plasmon modes (section 1.2, fig. $2 b)$.

Silver nanocubes (AgNCs) are a prime example of these plasmon modes as well as their enhancement upon approaching a dielectric substrate. In solution, AgNCs contain multiple modes owing to their sharp edges and vertices offering more positions for the electrons to dissipate to, generating high energy electric fields as a result of the charge accumulation. ${ }^{56}$ On the dielectric substrate, a different corresponding extinction spectrum is obtained. The hybridization of the dipolar $\left(\mathrm{D}^{0}\right)$ and quadrupolar $\left(\mathrm{Q}^{0}\right)$ modes to the hybrid dipole $(\mathrm{D})$ and quadrupole $(\mathrm{Q})$ mode occurs when the NC is in contact with the dielectric substrate.$^{53,56}$ In contrast, $\mathrm{D}$ is concentrated to the area of high refractive index (usually the dielectric substrate) whereas Q is confined to the surroundings. From this, it can be concluded that the D mode has higher sensitivity to changes in the substrates refractive index while Q is highly sensitive to changes in the surroundings (above the substrate). Consequently, we obtain modes that are both spectrally and spatially separated (fig. 9). ${ }^{17,57}$
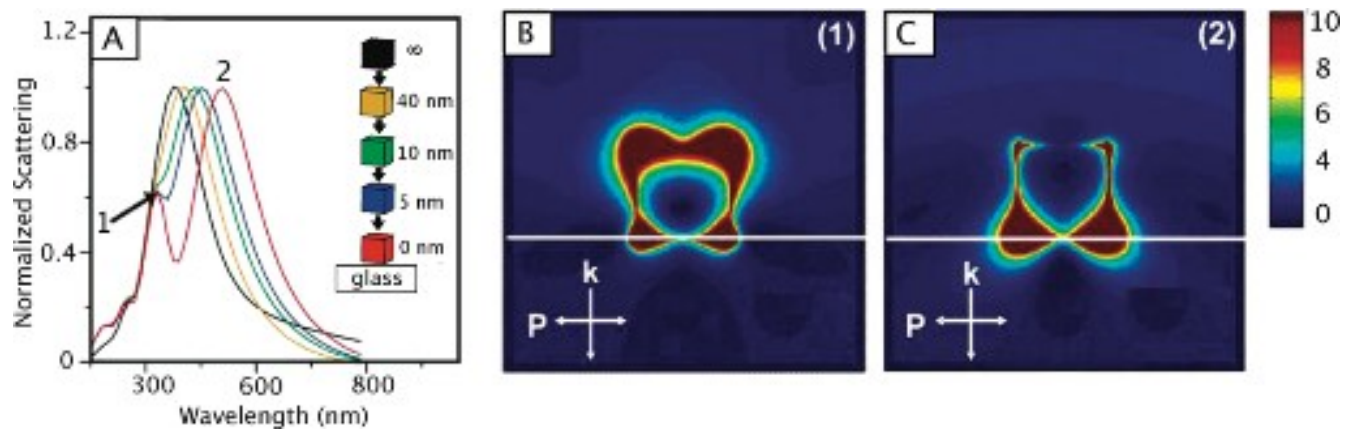

Figure 9: FDTD theory showing (A) the emergence of a second peak as a single nanocube (90-nm diameter) approaches a dielectric substrate, and $(B, C)$ the field intensities for peaks 1 and 2 of the nanocube in contact with the substrate (the white line in the field pattern images represent the substrate). Reprinted with permission from ref (57). Copyright 2013 ACS

Cubic nanoparticles have greater benefit over spherical nanoparticles upon interaction with a substrate as they occupy larger areas. ${ }^{40}$ Greater contact area of the particle with a substrate results 
in greater hybridization. Moreover, the edges and vertices of a cube, which allow for its many modes provide the ability for greater hybridizations via increased plasmon-surface interactions. ${ }^{53}$ The hybridization can also be said to originate from the similarities in the energies and charge distributions of both the $\mathrm{D}^{0}$ and $\mathrm{Q}^{0}$ modes.

\subsubsection{Gap Plasmons: Intentional Coupling of Surface Plasmon Resonances}

Coupling of nanoparticles to (i) other nanoparticles or (ii) a substrate has already been reported on and has shown the interesting and vast tunability of the optical properties of nanomaterials. Conventional nanoscience looks at the nanoparticle as an individual particle but has observed, in many cases, more complex systems than the classical solid metal, colloidal solution. Particle to particle plasmon coupling provides insight for further exploration of our ability to couple plasmonics to other substrates. ${ }^{48}$ Plasmonic particles can interact very strongly with thin metal films leading to a coupling band between them. ${ }^{58}$ This effect is very similar to interparticle coupling and can also be described using the plasmon hybridization model. ${ }^{59}$

A plasmonic nanoparticle brought within proximity to a metal surface (MS) can experience plasmon-plasmon coupling. ${ }^{59}$ The LSPR of a NP couples to the SP of a MS creating multiple cavity-like resonances. ${ }^{58}$ Using a plasmon hybridization model as well as the image interactions between the materials, a description of these multiple cavity-like resonances (gap modes) can be given. ${ }^{59}$ An image interaction is induced by the LSPR coupling to its projected charge image on the MS, and the charge image of any of surrounding LSPR sources of the same symmetry (fig. 10a). ${ }^{44}$ Plainly, the NPs charge distribution incites a local charge distribution of opposite sign within the MS. 

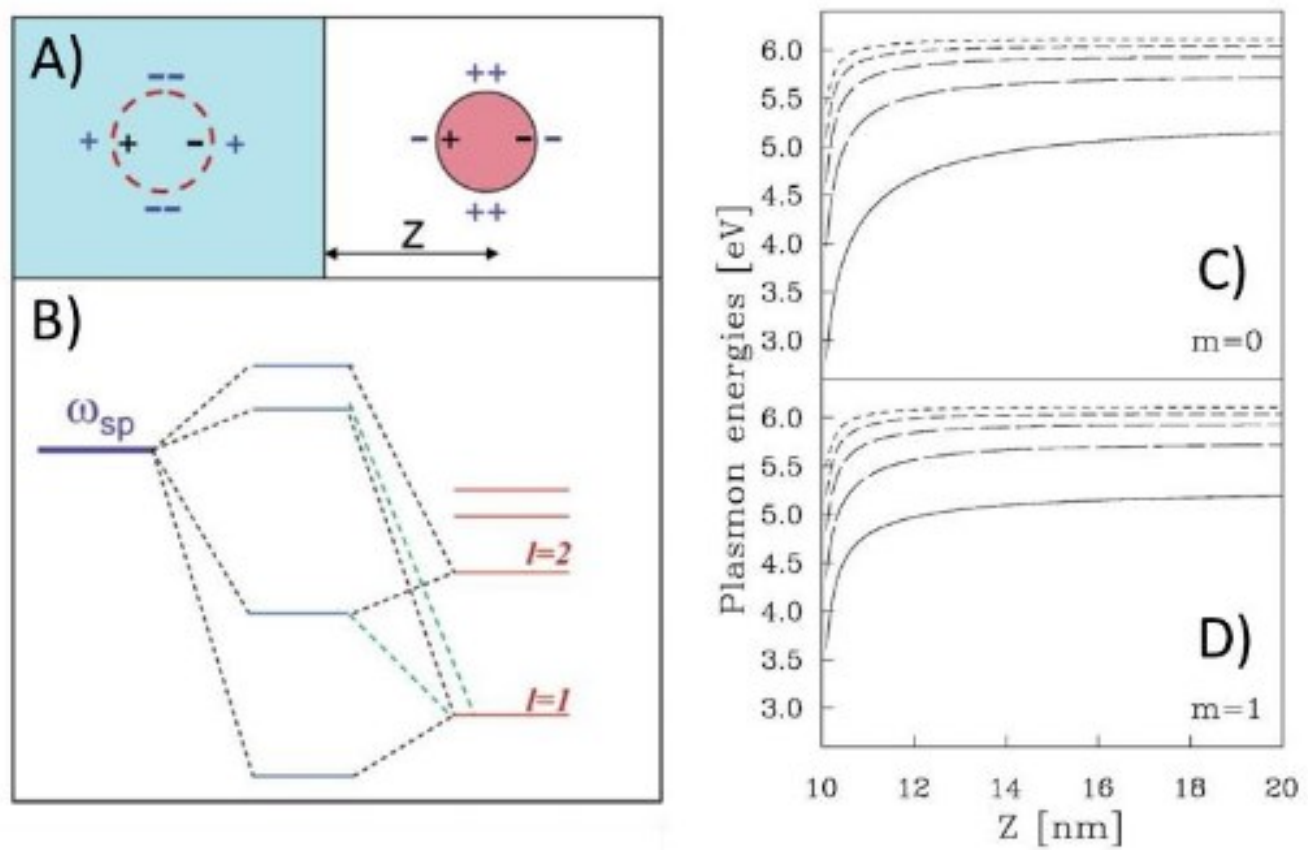

Figure 10: A) Image Interaction of Nanosphere with Metallic Substrate, B) Plasmon Hybridization Model, C) Perpendicular Dipole Mode, D) Parallel Dipole mode. Reprinted with permission from (59). Copyright (2004) American Chemical Society.

The plasmon hybridization model, fig. $10 \mathrm{~b}$, is very similar to that of a plasmon hybridizing with a dielectric substrate with the caveat being the NPs LSPR coupling to the MS SPs. A NPs distinct plasmon modes, having angular momentum $l=n$ (where $n=1,2,3,-, n$ ), increase in resonance frequency as the angular momentum is changed at the surface (e.g., atomic orbitals increasing in energy). ${ }^{59}$ Within the plasmon hybridization model, the surface plasmon mode is analogous to a density of state continuum $($ fig. $10 \mathrm{c}, d){ }^{59}$ the plasmon modes have an energy distribution dictated by the initial energy of the discrete plasmon modes. From this energy distribution, it is observed that as the LSPR containing NP is brought closer to the MS the plasmon energy dramatically increases meaning both perpendicular $(\mathrm{m}=0)$ and parallel $(\mathrm{m}=1)$ dipole can couple to the MS. Obviously, the perpendicular dipole experiences a larger change in energy (stronger interaction) as it approaches the MS and couples sooner than the parallel dipole. At far distances (i.e., $20 \mathrm{~nm}$ ) coupling is dominated by the image interaction. ${ }^{59}$ As the NP approaches the 
MS and the separation decreases, hybridization occurs and the plasmon mode's energy is stabilized. Lowering of the plasmon energy results in a spectral red shift of the gap modes. Importantly, at smaller separation distances higher energy plasmons will begin to couple to the SPs.
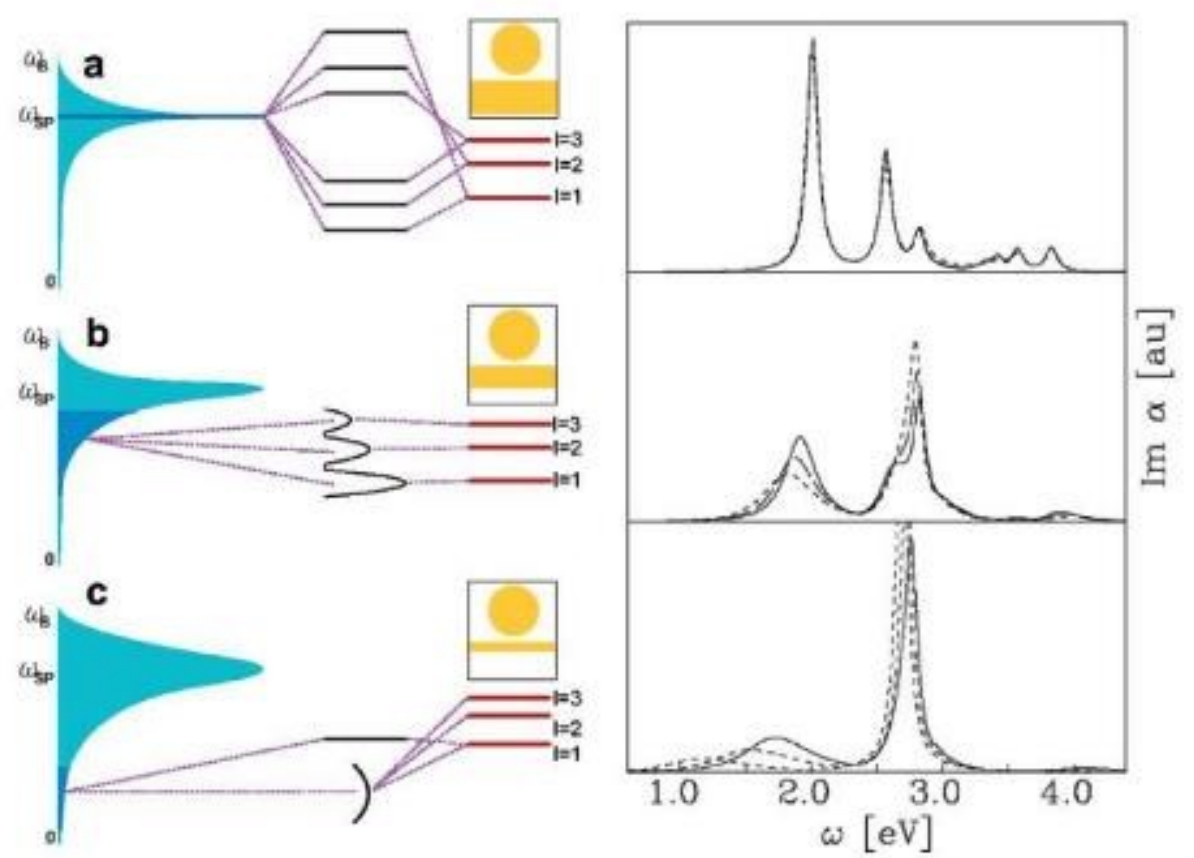

Figure 11: Coupling of Nanosphere LSPR to SP on metallic surface with a thickness of a) 400-100 nm, b) 25-16 nm, c) 12-4 nm. Reprinted with permission from (60). Copyright (2005) American Chemical Society.

The MS's thickness is also an important parameter to consider when manufacturing materials with gap plasmons. In the context of hybridization, SPs at the MS can be thought of as incompressible deformations of electron gas over a uniform distribution of positive charge carriers on the MS. ${ }^{59}$ Bulk plasmon frequency $\left(\omega_{B}\right)$ can be measured using eqn. 13 with the provided parameters: the number of electrons $\left(\mathrm{n}_{\mathrm{o}}\right)$, elementary charge $(\mathrm{e})$, and the mass of an electron $\left(\mathrm{m}_{\mathrm{e}}\right)$. This representation works well for approximating infinitely thick metal films but is not adequate for approximating thin metal films $(<100 \mathrm{~nm})$. Instead, an equation which incorporates the 
thickness of the metal film (T) and the incoming wavevector (k) is introduced (eqn. 14) to predict the plasmon frequency of thin films. ${ }^{60}$ Together these equations imply that i) surface plasmon energy is lower than bulk plasmon energy and ii) gap mode hybridization is influenced by film thickness.

$$
\begin{gathered}
\omega_{B}=\sqrt{\frac{4 \pi n_{o} e^{2}}{m_{e}}} \\
\omega_{\vec{k}, \pm}=\left(\frac{\omega_{B}}{\sqrt{2}}\right) \sqrt{\left(1 \pm e^{[-k T]}\right)}
\end{gathered}
$$

It has previously been shown that with AgNCs atop a metal film, separated by a thin polystyrene layer, a strong gap mode is observed (fig. 12). Initially, the gap distance is $20 \mathrm{~nm}$ and the induced charge at the metal is weakly interacting. This is a strongly coloured material. Once the particle is brought close to the MS, there are distinct red-shifts in the reflectance spectra. At a $5 \mathrm{~nm}$ gap a very strong interaction between the $\mathrm{AgNC}$ and metal surface is observed, producing a strong plasmonic response in the reflectance. It is hoped that by using mixtures of $\mathrm{Au}$ and $\mathrm{Ag}$ nanoparticles, the mode observed for silver will be obtained along with a second gap mode for $\mathrm{Au}$, generating a bounty of new colours.
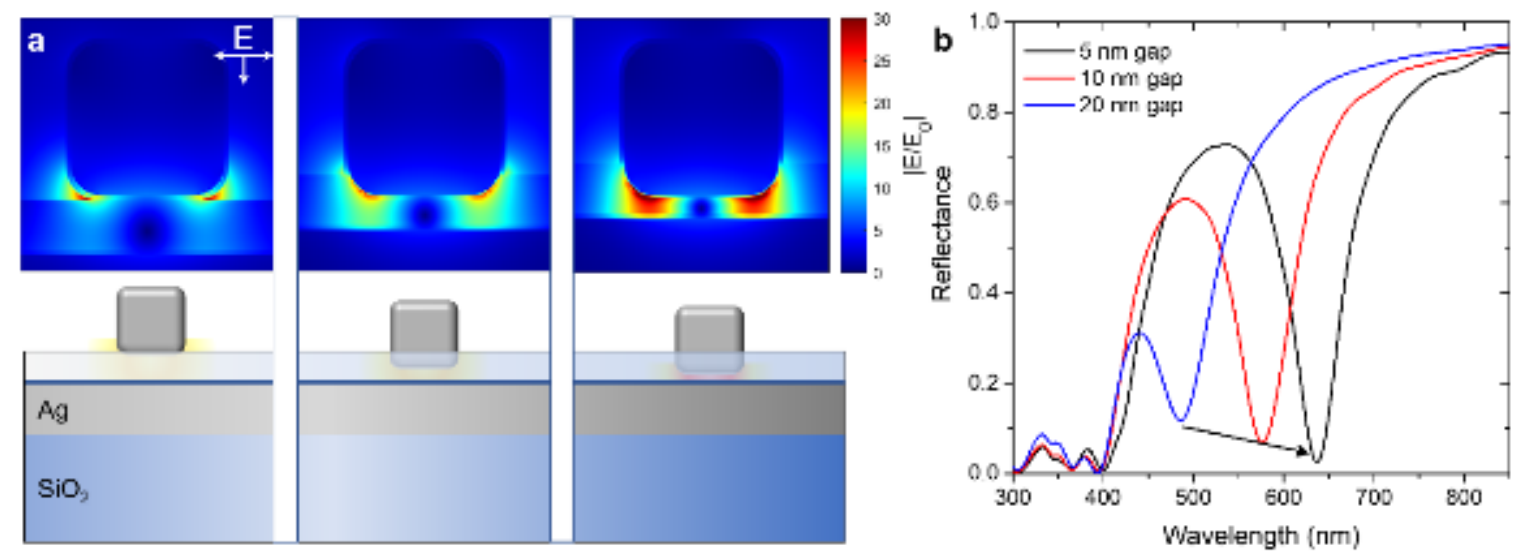

Figure 12: a) Electric field intensities of $60 \mathrm{~nm}$ Ag nanocubes over an Ag film separated by a dielectric spacer. The electric field intensity increases significantly when the particle is brought within $5 \mathrm{~nm}$ of the film. b) associated reflectance calculations showing a significant shift in the gap plasmon 


\subsubsection{Thermoplasmonics}

Irradiation of a plasmonic nanoparticle with light results in the excitation of the electrons contained in the conduction band of the metal. These excited electrons undergo electron-electron and electron-phonon collisions within the metal lattice. ${ }^{61}$ Hence, the energy absorbed by the plasmon is thermally dissipated (occurring on the order of picoseconds).
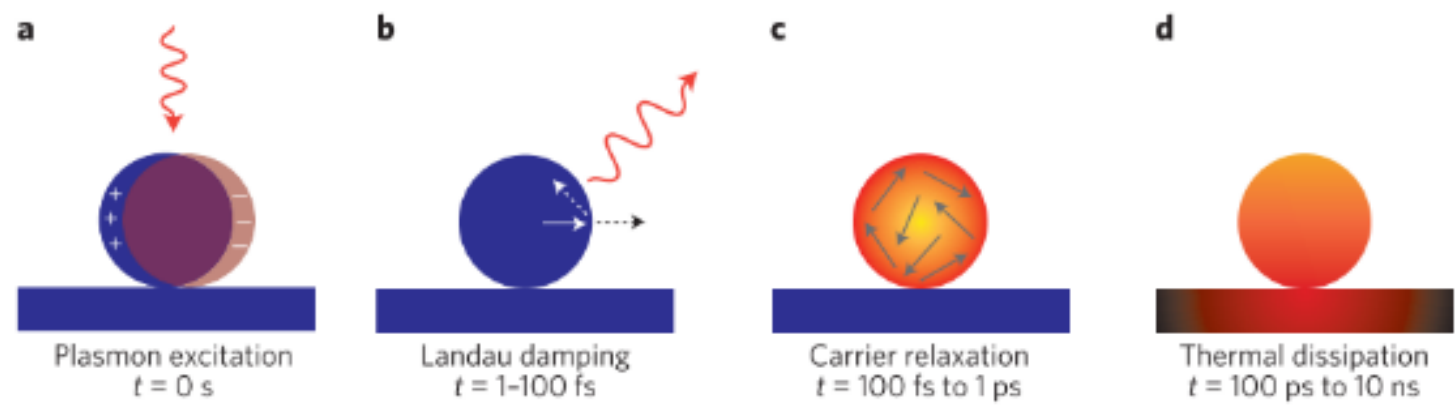

Figure 13: Schematic representation of the lifetime of a plasmon resonance. Upon the plasmon's excitation (a) the excited carriers undergo Landau damping (b) forming a thermal population of hot carriers (c) which subsequently relax and produce heat (d) through collisions in the lattice. Reproduced with permission, (C) Springer Nature publishing group, 2015 ref ${ }^{25}$

Nanoparticles have high thermal conductivity which results in the quick dissipation of heat to the surroundings. ${ }^{62}$ Within nanoseconds, the NPs release heat until they reach steady-state temperatures with the surroundings. Known as the thermoplasmonic effect, the nanoparticles can undergo rapid photoinduced heating with subsequent thermalization. This photothermal process can be used to localize heat at sub-diffraction limit dimensions. ${ }^{63}$ Depending on the power of light absorbed $\left(\mathrm{P}_{\mathrm{abs}}\right)$, the temperature increase $(\Delta \mathrm{T})$ of a plasmonic NP can be calculated. ${ }^{63}$

$$
\Delta T=\frac{P_{a b s}}{4 \pi r \kappa}=\frac{\sigma_{a b s} I}{4 \pi r \kappa}
$$

This expression signifies a direct correlation between $\mathrm{P}_{\text {abs }}$ and $\sigma_{\mathrm{abs}}$; the power of light absorbed by a NP is proportional to the absorption cross-section at the excitation frequency. NPs are beneficial here, as they have extremely high $\sigma_{\text {abs }}$ (often several times larger than the physical cross-section). ${ }^{64}$ The temperature increase is also a function of the nanoparticle radius (r), material 
thermal conductivity $(\kappa)$, and the irradiance of incident light (I). Heating can also be accomplished by excitation of the interband transitions within NPs, but is not a plasmon-induced phenomenon. ${ }^{65}$ A system containing multiple plasmon modes allows for the fine-tuning of the photothermal response of the NPs as the temperature increase is dependent on $\sigma_{\text {abs. }}{ }^{66}$ Depending on the shape, size and nature of the plasmon modes, there is different contributions of $\sigma_{\text {abs }}$ and $\sigma_{\text {scat }}$ to the extinction cross section. ${ }^{61}$ As well, the shape of NPs (i.e. their plasmonic response) can be altered using the thermoplasmonic effect. ${ }^{67}$

For more complex NP systems, the shape and size are important parameters to consider in the effective temperature increase. Baffou et al., presented an equation which uses the dimensionless shape correcting coefficient, $\beta$, and the radius of a sphere $\left(\mathrm{R}_{\mathrm{eq}}\right)$ with the same volume of the particle. ${ }^{63}$ With these parameters, the equation becomes:

$$
\Delta T=\frac{P_{a b s}}{4 \pi R_{e q} \beta \kappa}=\frac{\sigma_{a b s} I}{4 \pi R_{e q} \beta \kappa}
$$

Given that plasmonic nanoparticles have extremely high absorption cross-sections (up to four times greater than the geometric radius of the particle), ${ }^{23}$ the candidacy of these NPs for photothermal heating is promising. Though this representation works well for individual particles, it does not take into consideration the effect of simultaneous optical excitement of arrays of nanoparticles. ${ }^{68,69}$ The selected NP should have parameters which have been maximized to output the highest photothermal conversion. ${ }^{68}$ Regarding the size of the nanoparticle, it has been proven that as the NP size is increased there will be greater photothermal conversion, though particles above the threshold NP size will not have all of their volume contributing to photothermal heating. ${ }^{69-71}$

$$
J_{o}=\frac{\sigma_{a b s} \lambda_{r e f}}{2 \pi V}
$$


The $\sigma_{\text {abs }}$ of small NPs can be normalized by its volume (V) to isolate the effects of shape and composition elucidating the joule parameter $(\mathrm{Jo})($ fig. 14$) .{ }^{72} \mathrm{Here}, \lambda_{\text {ref }}$ is set arbitrarily to $\approx 1240 \mathrm{~nm}$ so that $J o$ is dimensionless. While other methods divide the $\sigma_{a b s}$ by the projected surface of the nanoparticle to obtain a dimensionless number that can be used for quantifying absorption efficiency, using Jo is advantageous as it works for any nanoparticle geometry and not solely spheres. $^{72}$ This equation confirms the efficiency of Au- and AgNPs for near-field enhancement and photothermal conversion - especially observed for Ag.
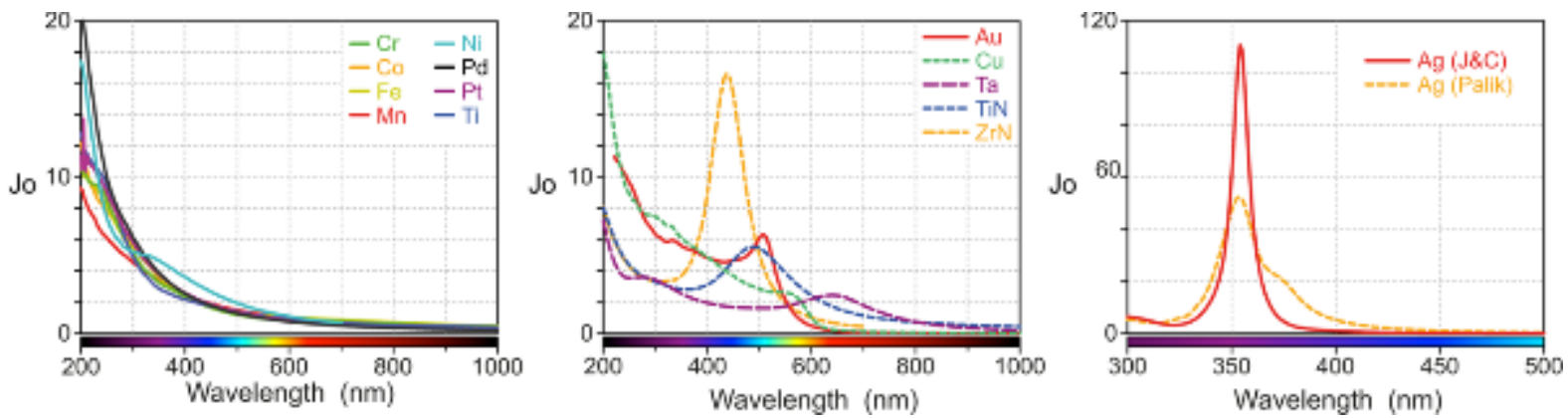

Figure 14: Spectra of the Joule numbers for a large set of materials. Materials have been arranged in families: Plasmonic resonance out of the visible range (left), in the visible range (middle), and the singular case of silver (right) where data have been plotted considering two distinct data sets (the optical constants of Johnson and Christy, and of Palik's handbook). Adapted with permission from (72).

Copyright 2015 ACS

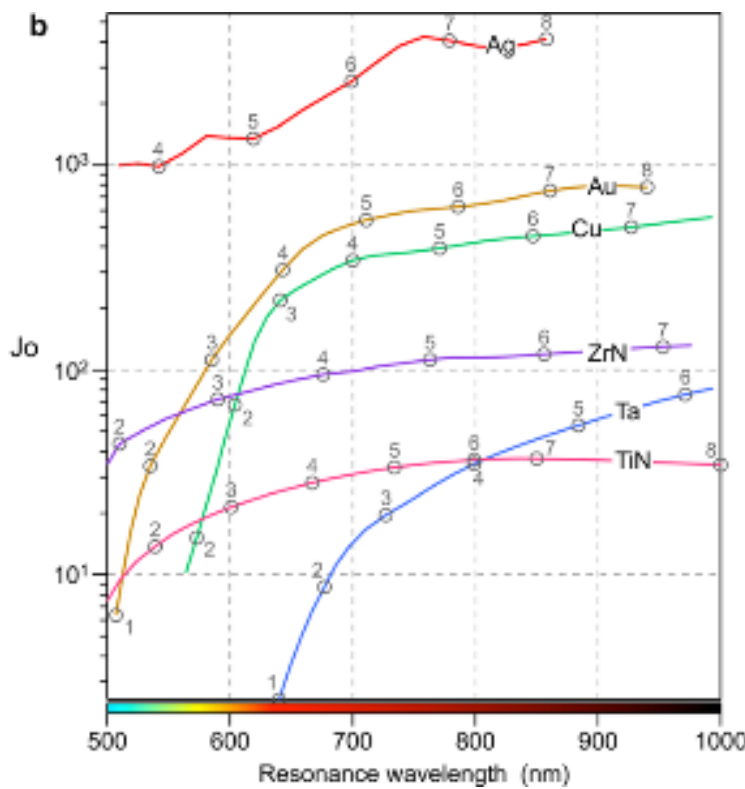

Figure 15: Calculated Jo number plotted as a function of the resonance wavelength of ellipsoids made of various materials. Adapted with permission from (72). Copyright 2015 ACS 
As well, it is observed that increasing the aspect ratio of the NP, fig. 15, will cause an increase in the joule parameter. Elongated particles have LSPRs which are more red-shifted and can even achieve higher order modes. These red shifted particles have longer wavelengths of light and hence require longer wavelengths for their excitation and subsequent thermoplasmonic heating..$^{70,72,73}$ Of course, the like would be considered for any particles at longer wavelengths, inclusive of NP dimers. ${ }^{74,75}$

\subsection{Nanoparticle Synthesis}

\subsubsection{Nanoparticle Stabilization}

The nanoparticle surface is stabilized (or capped) by a material which coats the surface and acts to prevent metal-metal interactions. Uncapped metals have increased opportunity for metalmetal interactions due to high probability for bare metals to form bonds leading to aggregation of nanoparticles. Aggregates are typically undesired formations that can restrict the usability of the colloidal suspension and can reduce the ability to form evenly distributed nanoparticle monolayers. Another result observed in aggregation is interparticle coupling (previously described). In order to mitigate these issues, many different stabilization agents have been utilized with their basis being upon either coulombic repulsion, or steric hinderance. ${ }^{32}$ Applying a charged material (e.g., citrate) can be used to cause coulombic repulsion in its purist form; likely charged particles with repel each other. ${ }^{76,77}$ Sterically, the particles can be blocked by binding bulky materials (e.g. large polymer chains) to the NP surface such that no metal to metal interactions can occur. ${ }^{78}$

\subsubsection{The Polyol Method: AgNCs Synthesis}

The polyol synthetic process is one involving a heated polyol (typically ethylene glycol or diethylene glycol) in the presence of a metal precursor salt (e.g., AgNO3 or HAuC14). ${ }^{20}$ Using the polyol process, varying shapes and sizes of nanoparticles can be synthesized based on the selection of the stabilization agent, as well and shape and growth directing agents. ${ }^{20,43,79}$ Ethylene glycol (EG) remains one of the most common polyols used for the synthesis of metal nanoparticles, acting 
as both the solvent and reducing agent. ${ }^{80}$ Heating EG above its threshold temperature $\left(120^{\circ} \mathrm{C}\right)$ in the presence of oxygen result in its oxidation to glycolaldehyde (GA). ${ }^{80}$ The amount of metal precursor produced is directly correlated to the temperature of EG, hence the concentration of GA. The reducing agent is continuously generated throughout the synthesis leaving no shortage of metal precursor for NP formation. ${ }^{80}$

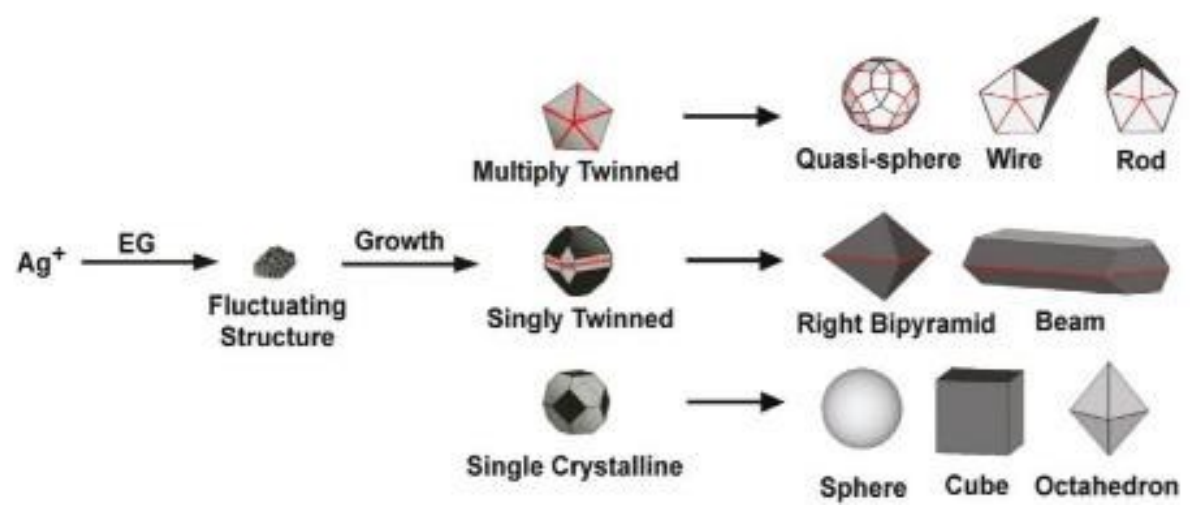

Figure 16: Shape-controlled synthesis of silver nanostructures. Ag nuclei will grow into either single crystals, or crystals containing single or multiple twin defects. Passivating agents can then be used to select the final morphology of the particle. Reproduced with permission from ref 81. Copyright 2007 ACS

With sufficient reducing agent availability, the precursor salt is reduced to its atomic (solid) form. As the concentration of $\mathrm{Ag}_{(\mathrm{s})}$ increases beyond the minimum saturation concentration $\left(C_{\min }^{n u}\right)$, Ag atoms begin to self-nucleate, forming small clusters with lower Gibbs free energy than the supersaturated system. ${ }^{81}$ Subsequently, the $\mathrm{Ag}_{(\mathrm{s})}$ concentration declines rapidly below $C_{\min }^{n u}$ and self-nucleation ceases to occur. ${ }^{81,82}$ Small clusters then continue to increase in size until a critical size where the energy of activation to change the shape becomes too high and the cluster forms into a seed. ${ }^{82}$ These seed particles then grow into larger nanocrystals until the concentration reaches the nanocrystal solubility in solution $\left(\mathrm{C}_{\mathrm{s}}\right)$. This is shown in fig. 17 .

Reaction 1: $2 \mathrm{HOCH}_{2} \mathrm{CH}_{2} \mathrm{OH}+\mathrm{O}_{2} \rightarrow 2 \mathrm{HOCH}_{2} \mathrm{CHO}+2 \mathrm{H}_{2} \mathrm{O}$ 
Reaction 2: $2 \mathrm{AgNO}_{3}+\mathrm{HOCH}_{2} \mathrm{CHO}+2 \mathrm{H}_{2} \mathrm{O} \rightarrow 2 \mathrm{Ag}(\mathrm{s})+\mathrm{HOCH}_{2} \mathrm{COOH}+2 \mathrm{HNO}_{3}$

Reaction 3: $3 \mathrm{Ag}(\mathrm{s})+4 \mathrm{HNO}_{3} \rightarrow 3 \mathrm{Ag}^{+}+3 \mathrm{NO}_{3}^{-}+\mathrm{NO}(\mathrm{g})+2 \mathrm{H}_{2} \mathrm{O}$

At $\mathrm{C}_{\mathrm{s}}$, nanoparticle growth does not stop, instead smaller particles will dissolve in solution to maintain the equilibrium concentration. The redissolved atoms are now in excess and begin to deposit onto the surface of larger, more established particles. ${ }^{83}$ The growth of larger nanoparticles at the loss of smaller nanoparticles is known commonly as Oswald's ripening, driven by an overall reduction in surface energy of the particles. ${ }^{77}$ Oswald ripening can result in the broadening or narrowing of NP size distribution under their respective ripening conditions. ${ }^{84}$

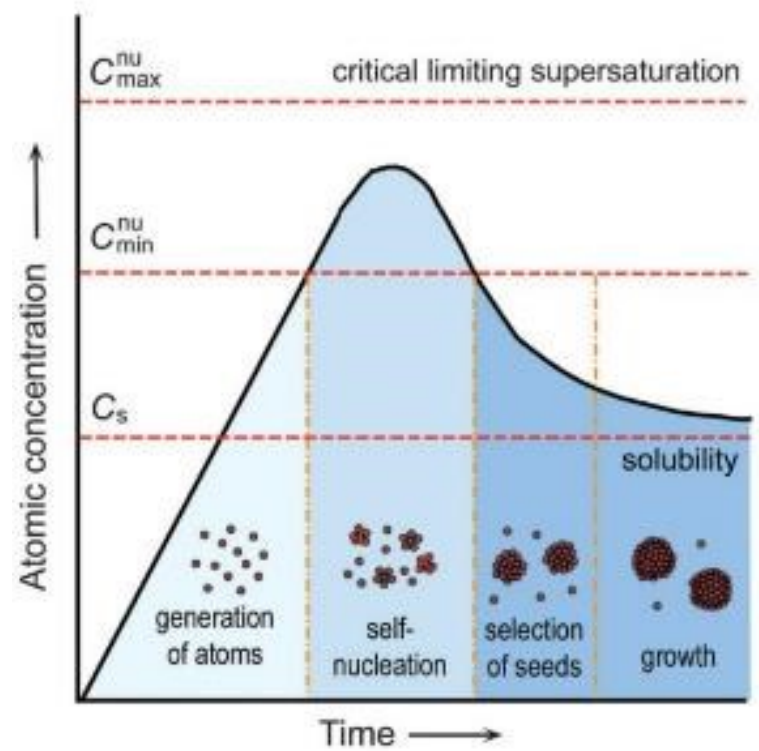

Figure 17: LaMer diagram of the growth process of colloidal crystals. After reduction of the precursor to $\mathrm{M}^{0}$ the solution becomes super-saturated with $\mathrm{M}^{0}$ initiating self-nucleation of the particles at a critical concentration $\left(\mathrm{C}_{\text {crit }}\right)$. Subsequent growth and agglomeration of the particles Reprinted with permission from ref 72. Copyright 2013 RCS and adapted from ref 71 (Copyright ACS 1950).

Within this process, oxidative etching of $\mathrm{Ag}$ by $\mathrm{HNO}_{3}$ to $\mathrm{Ag}^{+}$will also occur, etching away twinned seeds and reducing growth rate of NPs, thus increasing NP size distribution. ${ }^{80}$ To improve monodispersity of the nanoparticles trace amounts of $\mathrm{Na}_{2} \mathrm{~S}$ can be added to the system. $\mathrm{Na}_{2} \mathrm{~S}$ results in the production of $\mathrm{Ag}_{2} \mathrm{~S}$ nanoparticles which catalyze the formation of GA (increasing kinetic 
growth rate) ${ }^{85}$ Indirectly, the rate of reaction 3 is increased via increased production of $\mathrm{HNO}_{3}$ which results in the increase in the etching rate of twinned seeds. ${ }^{85}$ Thus, the amount of $\mathrm{Na}_{2} \mathrm{~S}$ needs to be optimized for $\mathrm{Ag}^{+}$concentration. At high ratios of $\mathrm{Na}_{2} \mathrm{~S}: \mathrm{Ag}^{+}$, the formation of high concentration of $\mathrm{Ag}_{2} \mathrm{~S}$ will proceed before the metal reduction can occur, these solid NPs have high stability and are insoluble in EG. If the $\mathrm{Na}_{2} \mathrm{~S}: \mathrm{Ag}^{+}$ratio is too low multiply twinned seed formation will occur.

\subsubsection{AuNP Synthesis}

Though there are many reducing agents that have been used for the synthesis of AuNPs, commonly the (i) Turkevich (Citrate) reduction method, and (ii) the Brust $\left(\mathrm{NaBH}_{4}\right)$ reduction method are used to reduce tetrachloroauric acid $\left(\mathrm{HAuCl}_{4}\right) \cdot{ }^{86,87}$ Depending on the desired geometry of the intended NP, typically one of the above methods is selected. These methods have evolved into further approaches to synthesis of gold nanoparticles (an important one being seed-mediated growth). ${ }^{87-89}$ Indeed, both reducing agents function for the synthesis of AgNPs as well. ${ }^{90,91}$ Herein, the seed-mediated growth of AuNPs is proceeded via citrate reduction. Citrate is beneficial as it is a weakly binding capping layer, especially in comparison to other commonly used surfactants like cetyl-trimethylammonium bromide $(\mathrm{CTAB}),{ }^{77}$ and can easily be replaced by other ligands (especially thiols).

The seed-mediated approach for AuNP growth is one of the most well-defined and used for research using AuNPs. ${ }^{92}$ For this approach, nanoparticle growth involves two-steps: seed nucleation followed by subsequent growth containing metal precursors, reducing and shapedirecting agents. The seed-mediated growth method is an example of a heterogeneous nucleation

processes ${ }^{93}$ Such growth benefits from its ease and precise tunability of parameters which directly affect the shape and evolution of particles with the desired size and morphology; greater control 
of size distribution. ${ }^{92}$ These parameters include changes in $\mathrm{pH}$, temperature, composition of growth solution and seed particle condition(s). ${ }^{92}$

\subsubsection{Reactions of Citrate and $\mathrm{HAuCl}_{4}$}

$\mathrm{HAuCl}_{4}$ can be hydrolyzed into various Au-complexes in a manner dependent on the $\mathrm{pH}$ of the reaction solution (reaction 4). ${ }^{94-97}$ Upon addition of a reducing agent [R], AuNPs are obtained and $\mathrm{Cl}$ and $\mathrm{OH}$ ions are released.

Reaction 4: $\mathrm{HAuCl}_{4}+\mathrm{nH}_{2} \mathrm{O} \rightleftharpoons \mathrm{AuCl}_{4-\mathrm{n}} \mathrm{OH}_{\mathrm{n}}^{-}+(\mathrm{n}+1) \mathrm{H}^{+}+\mathrm{nCl}^{-}$

Reaction 5: $\mathrm{AuCl}_{4-\mathrm{n}} \mathrm{OH}_{\mathrm{n}}^{-} \stackrel{[\mathrm{R}]}{\rightarrow} \mathrm{Au}_{(\mathrm{s}, \text { particle })}+(4-\mathrm{n}) \mathrm{Cl}^{-}+\mathrm{nOH}^{-}$

These reactions together are indicative of the dependence on the reducing power of the reducing agent, and the $\mathrm{pH}$ of the solution on the reduction rate of gold precursors. It is of importance to note that the $\mathrm{pH}$ alters the coordination state and activity of the Au precursors. ${ }^{94}$ Furthermore, the nucleation growth process and size of resulting NPs are also a function of reducing power and $\mathrm{pH} .{ }^{95-97}$ In water, and given the specific reducing agent - citrate, the hydrolysis of $\mathrm{HAuCl}_{4}$ and citrate can be provided: ${ }^{95}$

Reaction 6: $\mathrm{C}_{3} \mathrm{H}_{5} \mathrm{O}_{7}^{3-}+\mathrm{H}_{2} \mathrm{O} \rightarrow \mathrm{C}_{3} \mathrm{H}_{6} \mathrm{O}_{7}^{2-}+\mathrm{OH}^{-}$

Reaction 7: $\mathrm{AuCl}_{4}^{-}+\mathrm{OH}^{-} \rightarrow \mathrm{AuCl}_{3}(\mathrm{OH})^{-}+\mathrm{Cl}^{-}$

Reaction 8: $\mathrm{AuCl}_{3}(\mathrm{OH})^{-}+\mathrm{OH}^{-} \rightarrow \mathrm{AuCl}_{2}(\mathrm{OH})_{2}{ }^{-}+\mathrm{Cl}$

Reaction 9: $\mathrm{AuCl}_{2}(\mathrm{OH})_{2}{ }^{-}+\mathrm{OH}^{-} \rightarrow \operatorname{AuCl}(\mathrm{OH})_{3}{ }^{-}+\mathrm{Cl}$

Reaction 10: $\mathrm{AuCl}(\mathrm{OH})_{3}{ }^{-}+\mathrm{OH}^{-} \rightarrow \mathrm{Au}(\mathrm{OH})_{4}{ }^{-}+\mathrm{Cl}$

Depending on the $\mathrm{pH}$ of the solution $\mathrm{AuCl}_{4}^{-}$is hydrolyzed to the different auric precursor ions (reaction 7-10), with their reactive activities decreasing in order. ${ }^{95}$ Citrate is hydrolyzed and oxidized to sodium acetone dicarboxylate (SADC) and upon addition of $\mathrm{HAuCl}_{4}\left(\right.$ already $\left.\mathrm{AuCl}_{4}^{-}\right)$ 
to the solution it is simultaneously reduced to $\mathrm{AuCl}$. The redox reaction between citrate and $\mathrm{HAuCl}_{4}$ is provided in reactions 11 and 12.

Reaction 11: $\mathrm{C}_{3} \mathrm{H}_{5} \mathrm{O}_{7}^{3-} \rightarrow \mathrm{C}_{2} \mathrm{H}_{5} \mathrm{O}_{5}^{2-}(\mathrm{SADC})+\mathrm{CO}_{2}+\mathrm{H}^{+}+2 \mathrm{e}^{-}$

Reaction 12: $\mathrm{AuCl}_{3}+2 e^{-} \rightarrow \mathrm{AuCl}+2 \mathrm{Cl}^{-}$

After this redox process, SADC will complex with $\mathrm{AuCl}$ to form AuNPs, or it may decompose into acetone. The acetone will undergo a second redox reaction with $\mathrm{AuCl}_{3}$ to produce more $\mathrm{AuCl}$ resulting in secondary nucleation, increasing size distribution..$^{95}$ The $\mathrm{AuCl}$ will then disproportionate to elemental $\mathrm{Au}$ and more $\mathrm{AuCl}_{3}$ in a rapid auto-catalytic process catalyzed by nuclei formations. ${ }^{98}$ Unlike LaMer's theory, ${ }^{82}$ these nuclei are not formed in collision based processes, rather $\mathrm{SADC}$ and $\mathrm{AuCl}$ are coordinated into macromolecular complexes which consecutively coagulate into colloidally stable particles. ${ }^{12,95}$

The seed mediated growth process is one that is reliant on various conditions $-\mathrm{pH}$ and concentration - as well as temperature. Neus et al. have provided a systematic seeded approach to growth of AuNPs with high monodispersity which allows selective growth of smaller seed particles into the desired size of larger particles at much higher concentrations than previously reported. ${ }^{77}$ This approach allows for the growth of uniform quasi-spherical nanoparticles ranging in size from 10 to $\sim 180 \mathrm{~nm}^{.7}$

\subsubsection{Ligand Exchange}

The capping ligand adsorbs onto the surface of the NP, minimizing its surface energy and provides interparticle repulsion sufficient enough to curb aggregation, affording stable metal NPs. ${ }^{99}$ For their applications and understanding, it is fundamentally important to be able to control, (i) the size and composition of the NP core, and (ii) the chemical nature of the stabilizing ligand. ${ }^{100}$ The ligand shell allows for control over the solubility, chemical reactivity, surface chemistry and binding affinity. ${ }^{100}$ To create tailored nanoparticle samples, it is important that any synthetic 
method used be novel and convenient, in addition to just nanoparticles with well-defined structures. Ligand exchange reactions have shown to be a powerful approach moving directionally towards simpler fabrication methods. ${ }^{100}$ In most cases, ligand exchange reactions involve the replacement of original ligands with stronger surface binding molecules, such that that exchange is typically irreversible. ${ }^{101}$ Within, the ligand exchange is used to transfer citrate capped AuNPs to PVP capped NPs allowing for their increased organic hydrophobicity to be taken advantage of in the fabrication of Langmuir films.

\subsection{Nanomaterial Assembly}

\subsubsection{Polymer thin films}

A polymer is a macromolecule containing many repeating units of a compound. Polymer films have thickness dependent properties, making them of great interest in the fields of nano- and materials science. Crystalline polymers have distinct melting temperatures, but their amorphous counterparts have what is known as a glass transition temperature $\left(\mathrm{T}_{\mathrm{g}}\right)$. At $\mathrm{T}_{\mathrm{g}}$, the polymer will become an rubbery, viscous fluid-like material. ${ }^{102}$ The bulk polymer, below its $\mathrm{T}_{\mathrm{g}}\left(\sim 100{ }^{\circ} \mathrm{C}\right)$, is described as a glass that is more of less brittle depending on how cooled it is. ${ }^{102}$ A polymer film below $100 \mathrm{~nm}$ is well within the nano-regime, being classified as a "thin film". ${ }^{103}$ Thin polymer films have thickness dependent properties inclusive of refractive index, $\mathrm{T}_{\mathrm{g}}$, and film stability differing from that of the bulk counterpart. ${ }^{104,105}$ For example, values ranging between $75-107^{\circ} \mathrm{C}$ have been reported for thin polymer films. ${ }^{105,106}$ The thickness dependent properties of these thin films can be exploited in the formation of polymer nanocomposites (NP/polymer systems). Nanocomposite materials are useful in various applications, including but not limited to; catalysis, near-field spectroscopy and sensing especially when NPs are above the polymer, and by embedding NPs into the polymer, the polymers mechanical strength increases as well as thermal and electrical conductivity. ${ }^{107-109}$ 
Employing static spin coating enables the fabrication of thin polymer film, though simple, it is a reliable means to produce the desired thickness. Aqueous polymer is adsorbed onto the surface of the substrate and excess (unbound) polymer will be spun off the substrate at high speeds (RPM). The polymer is subsequently annealed to the substrate at temperatures high enough to evaporate the solvent to increase the uniformity and smoothness of the thin film. Importantly, the substrate needs to be left to dry before any annealing steps are proceeded otherwise solvent may evaporate from within the polymer leaving large holes within. The thickness obtained for these thin polymer films is dependent on many parameters inclusive of polymer properties (e.g., hydrophobicity or air stability), concentration, spin speed, acceleration rate, spin time and experimental precaution..$^{104,107,110}$

\subsubsection{Langmuir Films}

There are various methods used for deposition of nanoparticles onto a substrate. ${ }^{108}$ Such feats have been accomplished using layer-by-layer, self-assembled monolayers, Langmuir technique, block co-polymer templating, and many more deposition techniques. ${ }^{108,111,112}$ Of these techniques, Langmuir stands in high ranking owing to its relatively efficient and easy monolayer formation. Langmuir films take advantage of the dispersion of organic molecules onto an air/water interface enabling the formation of a monolayer with controllable particle spacing using a movable barrier. ${ }^{113}$ The newly formed monolayer can then be transferred to a substrate using either vertical dipping (Langmuir-Blodgett (LB)) or horizontal stamping (Langmuir-Schaffer (LS)). By changing the lateral surface pressure of the monolayer (i.e., the surface pressure that the particles apply upon each other) the interparticle space can be changed. ${ }^{34}$ Metal nanostructures benefit from the Langmuir technique as they usually are stabilized with organic molecules with hydrophobic components. This technique has been used to successfully deposit NPs of various shapes and sizes. ${ }^{111}$ 


$$
F=2(w+t)(S T) \cos \theta
$$

Langmuir films are controlled by isothermal compression, monitoring the surface pressure $\left(P_{\text {surf }}\right)$ using a Wilhelmy plate (piece of paper of specific dimensions) to monitor the pressure at the interface. The vertical force $(\mathrm{F})$ experienced by the Wilhelmy plate is a product of surface tension (ST), buoyancy and gravity. The surface tension term represents the displacement of the water molecules around the plate. Given that the plate is of a specific width (w) and height $(\mathrm{h})$, the gravity and buoyancy term become negligible simplifying the equation. The wetting angle $(\theta)$ is orthogonal to the water surface simplifying the equation w.r.t. ST. The surface pressure can then be related to ST, where $\mathrm{C}$ is a constant for the absolute surface tension of the liquid.

$$
\begin{gathered}
S T=\frac{F}{2(w+t)} \\
C=S T+P_{\text {surf }} \Rightarrow P_{\text {surf }}=C-S T
\end{gathered}
$$

This relationship shows that as ST decreases, $P_{\text {surf }}$ increases proportionally. As a Langmuir isotherm is processed, phases analogous to gas, liquid and solid are seen. ${ }^{114}$ The gas phase is associated with near zero pressure indicating the particles are well spaced, containing large free volumes and having minimal interactions with other particles. Increasing the pressure brings particles closer together causing more interparticle interactions as would be observed in a liquid. In this phase the isotherm is noticed to have a slight, curved slope. Often, a plateau is also observed and is a "sub-phase transition" from expanded and condensed liquid phases. ${ }^{114}$ This plateau occurs because of the reorganization of particles as their free volumes approach their dimensional (bulk) size. At high surface pressures, the free volume of particles is small enough for a vertical line to appear in the isotherm indicating that the solid-like phase has been reached and the material will act more as a bulk material. 
Deposition of the monolayer onto a solid substate can be conducted using LB or LS (fig. 18). Langmuir-Blodgett is the vertical dipping of a solid substate into the monolayer until submerged, then slowly removing the substrate from the meniscus. LB is concerned with the transfer of a monolayer to a hydrophilic surface (due to the requirement of a positive meniscus). ${ }^{115}$ Langmuir-Schaffer on the other hand is used for hydrophobic surfaces and monolayers. LS is the horizontal stamping of the substrate onto the nanoparticle-water interface, withdrawing a monolayer as it is retracted from the surface. ${ }^{115}$
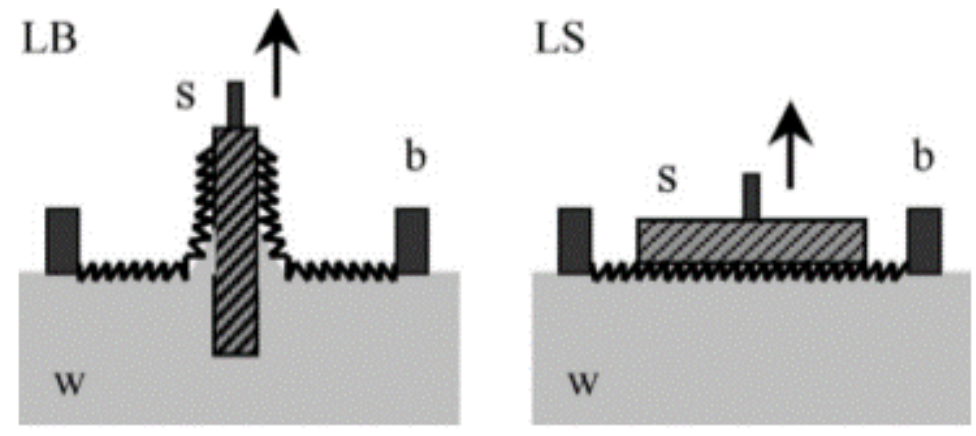

Figure 18: Langmuir film substrate transfer technique: Blodgett deposition (left) and Schaefer deposition (right). Reproduced from 115 Copyright Elsevier 2006.

\subsubsection{Nanoparticle Embedding into a Polymer Film}

A nanoparticle atop a polymer will begin to embed into that polymer film as the temperature of the material is heated to $T \geq T_{g}$. When $T \geq T_{g}$ the material becomes a rubber-like, malleable material - allowing the nanoparticle to sink into the polymer while the polymer maneuvers around the particle. Using a model that considers the Helmholtz free energy between the particle and the polymer film. ${ }^{116}$ This model assumes a spherical particle on a perfectly flat, infinite, polymer surface permitting no surface wetting layer exists. Helmholtz free energy (A) is a measure of the total work that can be done on a system barring temperature (T) and volume (V) are held constant. Equation 19 shows the change in Helmholtz free energy $(\Delta \mathrm{A})$ w.r.t. the change in total internal energy of the system $(\Delta \mathrm{U})$, temperature and entropy change $(\Delta \mathrm{S})$.

$$
\Delta A=\Delta U-T \Delta S
$$


Using the product of surface area (SA) and surface tension of the particle $\left(\gamma_{\text {particle }}\right)$, the surface free energy $\left(\mathrm{A}_{\text {surf }}\right)$ can be determined:

$$
A_{\text {surf }}=(S A) \gamma_{\text {particle }}
$$

As the particle embeds into the polymer, the surface tension term representing the interfacial tension between the particle surface and the polymer becomes $\gamma_{p s}$. The equation for the change in surface free energy of the embedded particle is then:

$$
\Delta A_{\text {surf }}=(S A)\left(\gamma_{p s}-\gamma_{\text {particle }}\right)
$$

Particle embedment can be thought of in two critical steps; i) Loss of surface energy as a "void" in the polymer is created, and ii) Gain of surface energy as the particle fills the void. ${ }^{116}$ The surface energy required to create a void in the polymer can also be described as the product of the particles surface area and surface tension of the polymer surface $\left(\gamma_{\text {surf }}\right) .{ }^{116}$ In contrast, the $A_{\text {surf }}$ which is gained by the particle filling the void is the product of the particle's SA and the work of adhesion between the polymer surface and the particle $\left(\mathrm{W}_{\mathrm{ps}}\right)$.

$$
\Delta A_{\text {surf }}=(S A)\left(\gamma_{\text {surf }}-W_{p s}\right)
$$

These two expressions can be cooperated to provide the $\mathrm{W}_{\mathrm{ps}}$ in terms of surface tension $(\gamma)$. For embedment to occur spontaneously, there must be a negative Helmholtz free energy $(\Delta \mathrm{A}<0)$. With this given boundary condition, the equation can be written as a relationship that is true for spontaneous changes in the particle-polymer motif.

$$
\begin{gathered}
W_{p s}=\gamma_{\text {surf }}+\gamma_{\text {particle }}-\gamma_{p s} \\
\gamma_{\text {surf }}<W_{p s}, \quad \Delta A<0 \\
\gamma_{\text {surf }}<\gamma_{\text {surf }}+\gamma_{\text {particle }}-\gamma_{p s} \\
\gamma_{\text {particle }}>\gamma_{p s}
\end{gathered}
$$


Though this relationship provides a good general description for the surface tension requirements of particle embedment into a polymer, it does not aid detail about the embedment process itself. During embedment, a new polymer surface is created with an area equal to the particle that has been removed from air. This provides a new relationship to describe to the surface tension condition for spontaneous embedment: $\gamma_{\text {particle }}>\gamma_{\text {surf }}+\gamma_{p s}$. This allows for the explanation of the thermal embedment of NPs into a thin polymer film. Thus, as the polymer is heated to its $T_{g}$, the surface tension of the polymer is lowered resulting in the formation of a void and concurrently filling of the void with a NP. ${ }^{116}$ As the process proceeds, the void is able to grow larger and the particle can embed to greater depth into the thin film, doing so until the surface tension of the particle is at or lower than the interfacial, and polymer thin film surface tension:

$$
\gamma_{\text {particle }} \leq \gamma_{\text {surf }}+\gamma_{p s}
$$

These surface tension parameters are reliant on the interaction of the materials at the interface. Within this research the interaction is facilitated through use of two polymers; poly(styrene) used to generate thin films through static spin coating and poly (vinyl pyrrolidone) as the stabilization agent.

\subsection{Describing Plasmonic Systems and Materials}

\subsubsection{Finite-Difference Time Domain (FDTD) Modelling}

Using classical electrodynamics, plasmonic systems can be described in terms of the propagation of EM waves through different components and materials. Maxwell's equations can used to determine the evolution of electromagnetic fields, and for a system containing no naturally magnetic components become: ${ }^{117}$

$$
\begin{aligned}
& \frac{\partial \vec{E}}{\partial t}=\frac{1}{\varepsilon_{0}} \nabla \times \vec{H}, \\
& \frac{\partial \vec{H}}{\partial t}=\frac{1}{\mu_{0}} \nabla \times \vec{E}
\end{aligned}
$$


where $\vec{H}$ and $\vec{E}$ are the electric and magnetic fields respectively, $\nabla x$ is the curl operator acting upon the field, $\varepsilon_{0}$ and $\mu_{0}$ are the permittivity (measure of electric polarizability of a dielectic) and permeability (magnetic dipolar response to an applied magnetic field) of free space respectively, while $\varepsilon_{r}$ is the relative permittivity and is a function of frequency, $\omega$. A challenge that arises is solving these equations over an arbitrary space where the materials (i.e., $\left.\varepsilon_{r}(\omega)\right)$ vary depending on spatial location. Typical methods would require tedious solvation of Maxwell's equations on incremental spaces of the object, but this is obviously mathematically insensitive especially when solving over large three-dimensional (3D) objects. Hence, useful computational methods have been designed for the modelling more complex systems. Of these techniques FDTD is one of the most commonly used, ${ }^{118}$ but other frequency domain techniques such as the finite element method, ${ }^{119}$ and discrete-dipole approximation ${ }^{120}$ method have been used. FDTD is very useful and well-established method for modelling of plasmonic systems. ${ }^{121,122}$

In 1965, Yee published a proposition of the discrete solutions to Maxwell's equations based on central difference approximations of the spatial and temporal derivatives of the curl equations. ${ }^{123}$ Now known as a Yee cell, fig. $19 b$, the spatiotemporal grid provided methodology to take the spatial and temporal segments and apply Maxwell's to solve the various field vectors at discrete points. ${ }^{122-124}$ 


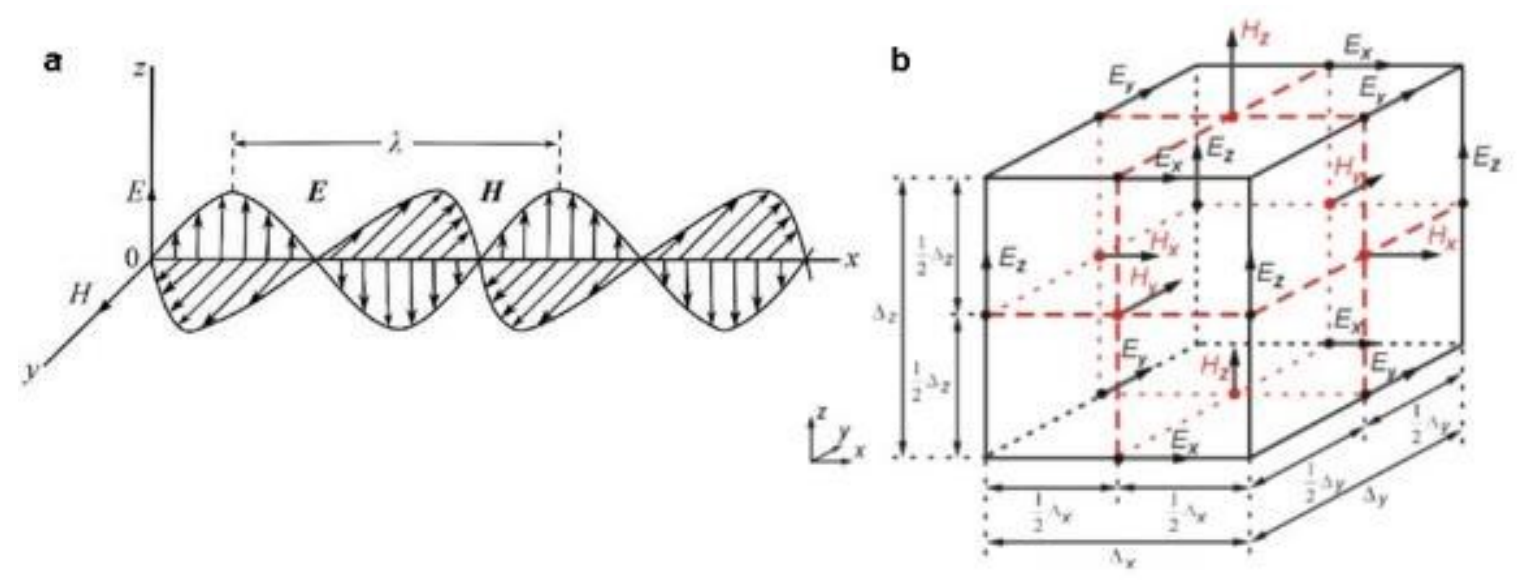

Figure 19: a) Schematic diagram of an electromagnetic wave showing the electric $(E)$ and magnetic $(H)$ components and $\mathrm{b}$ ) a diagram of the Yee cell, where $\mathrm{E}(\mathrm{t})$ and $\mathrm{H}(\mathrm{t})$ are offset in half-steps to propagate fields. Reproduced from ref 127 under open access CC license 4.0.

An FDTD solver discretizes the simulation space into a 2D or 3D mesh in cartesian coordinates, then solves Maxwells equations using a new form of equations: ${ }^{117,125}$

$$
\begin{gathered}
\frac{\partial \vec{D}}{\partial t}=\nabla \times \vec{H} \\
\vec{D}(\omega)=\varepsilon_{0} \varepsilon_{r}(\omega) \vec{E}(\omega) \\
\nabla \times \vec{E}=-\mu_{0} \frac{\partial \vec{H}}{\partial t}
\end{gathered}
$$

where $\vec{D}$ is the displacement field. Note that $\vec{D}$ is written in the frequency domain, though it must be written to the time domain for implementation in FDTD. ${ }^{117}$ Each equation becomes interdependent and cyclic in this form, and the use of $\vec{D}$ allows for the implementation of the complex dielectric function $\varepsilon_{r}(\omega)$. Being cyclic, these equations are the basis of the establishment of the Yee cell. in which solutions of the equations are propagated in time and space. In this scheme, the vectors of $\mathrm{E}$ and $\mathrm{H}$ are separated into six components $\left(\vec{E}_{x}, \vec{E}_{y}, \vec{E}_{z}\right.$, and $\left.\vec{H}_{x}, \vec{H}_{y}, \vec{H}_{z}\right)$, where the $\vec{E}$ and $\vec{H}$ components are discretized into separate grids which are offset by a half-step in time and space (Figure 1.3b). Propagation of the fields thus occurs in 'leapfrog' fashion where 
$\vec{E}(\mathrm{t})$ solves for $\vec{H}(\mathrm{t})$ a half-step forward, and $\vec{H}(\mathrm{t})$ solves for $\vec{E}(\mathrm{t})$ to complete a full step. The number of cells determine the computational cost of the model.

For materials such as metals, with rapidly changing electric and magnetic fields, fine meshing $(0.5-1 \mathrm{~nm})$ is necessary for computational accuracy. Commercial software, like Lumerical FDTD solver, can study and model small nanoassemblies, and make doing so convenient enough to be used on a common desktop computer. More advanced models come at high computational cost and require more powerful computers. The software uses semi-empirical dielectric functions (a model of the dielectric functions fitted to experimental data) to predict how the system will interact with the chosen light sources. Varying shape, size, material, dielectric environment, and direction and type of incident light allows for use of FDTD simulations to determine how the optical and electronic properties of a nanostructure are impacted by these changes.

These simulations are great for providing a model of the different nanomaterials and the responses to incident light. Though it is important to note that there can be discrepancies between the experimental result and the theoretical model. ${ }^{126-129}$ Often discrepancies between experimental and computation methods arise from properties such as size distribution, geometric parameters, as well, the dielectric functions of the actual materials used may not be identical to values inputted or estimated by the solver. ${ }^{126,129}$ The light source imparted onto the system, whether experimental or theoretical is another parameter of crucial importance. ${ }^{130}$

\subsubsection{Representing Colour}

Naturally, the study of colour (colourimetry) requires a systematic approach for representing, comparing, and quantifying colour. In 1931, the Commission international de l'eclairage (CIE) established a system of equations to map a colour space and consistently describe colour. The CIE 1931 colour space is a long-standing standard used in colourimetry. Fig. 20a, is the CIE colour space, and inset SRGB (reg-green-blue) colour space used in most displays and 
standard printers. The system of equations, known as the tristimulus functions $(\mathrm{x}, \mathrm{y}, \mathrm{z})$, transform spectral data (including reflection, transmission, scattering) into the CIE colour space. These tristimulus functions (fig. 20b) are a model of the spectral sensitivity of three types of human cone cells that perceive red, green, and blue light respectively. ${ }^{131}$
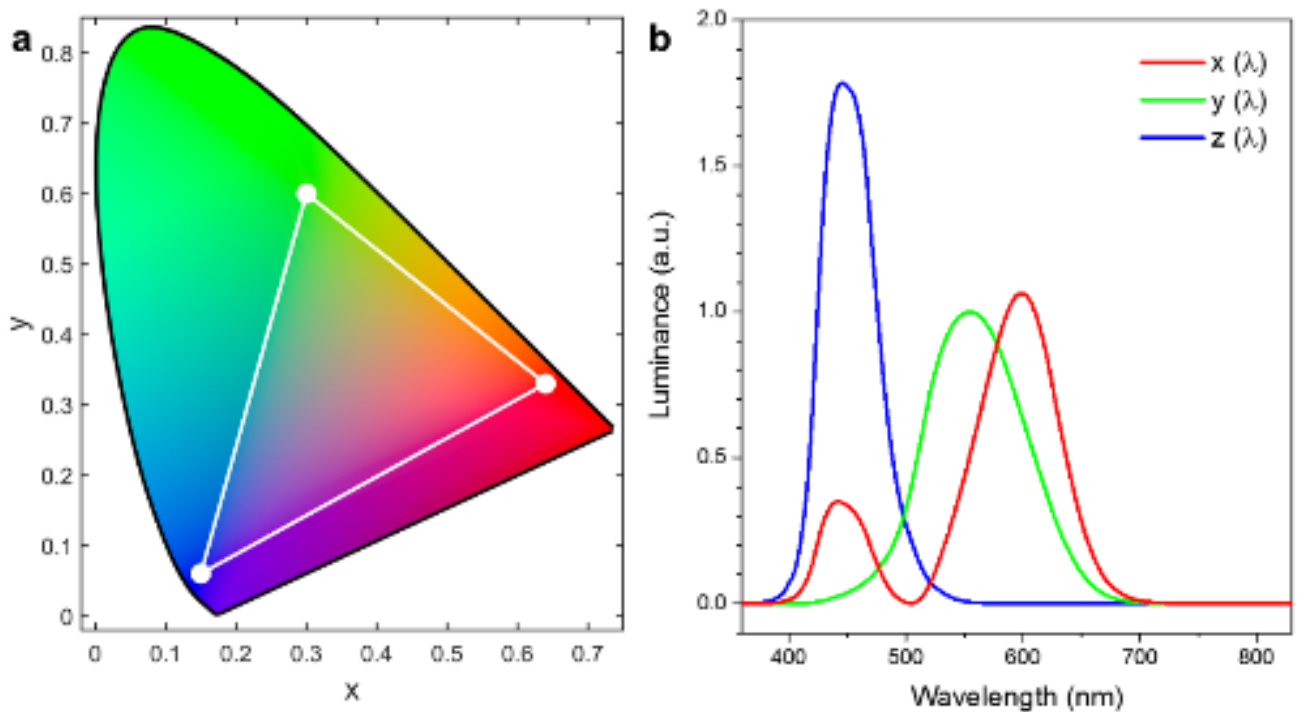

Figure 20: a) plot of the CIE 1931 colour space with, with the sRGB colour space shown within. Colours outside the triangle cannot be rendered properly on your display b) The tristimulus colour matching functions, which model the sensitivity of the human eye to red, green, and blue light.

Though there are three tristimulus functions, the values are typically normalized and displayed two-dimensionally (2D) for ease of viewing, rendering the third coordinate redundant. ${ }^{132}$ Consequently, information on brightness is lost when using the 2D colour space. Spectral reflectance $(\% \mathrm{R})$ of transmittance $(\% \mathrm{~T}), \mathrm{I}(\lambda)$ data can be converted to CIE XYZ values using the colour matching equations:

$$
\begin{aligned}
& X=\frac{1}{N} \int_{\lambda_{1}}^{\lambda_{2}} S(\lambda) I(\lambda) \bar{x}(\lambda) d \lambda \\
& Y=\frac{1}{N} \int_{\lambda_{1}}^{\lambda_{2}} S(\lambda) I(\lambda) \bar{y}(\lambda) d \lambda
\end{aligned}
$$




$$
\begin{gathered}
Z=\frac{1}{N} \int_{\lambda_{1}}^{\lambda_{2}} S(\lambda) I(\lambda) \bar{z}(\lambda) d \lambda \\
N=\int_{\lambda_{1}}^{\lambda_{2}} I(\lambda) \bar{y}(\lambda) d \lambda
\end{gathered}
$$

Here, $\bar{x}(\lambda), \bar{y}(\lambda)$, and $\bar{z}(\lambda)$ are the tristimulus functions and $\mathrm{S}(\lambda)$ is the illuminant function. The illuminant function is a representation of the light source of the view colours and is required for $\% \mathrm{~T}$ and $\% \mathrm{R}$ data as it provided a subjective bias to the observed colour. There are several illuminant functions to describe different light sources, but the most common is the "D65" illuminant as it simulates solar radiation with a colour temperature of $6500 \mathrm{~K}$. This source mimics daylight conditions in the northern hemisphere and accounts for atmospheric scattering.

\subsection{Rationale and Hypotheses}

In the many curiosities of life, light was found to be more than just a steady beam of white, rather an expansive gamut of colours was contained within. Since the inceptual understandings of EM radiation, scientists have explored many ways to control and manipulate electromagnetic waves to harness that energy for photocatalysis, production of photovoltaics, or fibre optics among many other applications. Although, limitations exist in the efficiencies of these materials and processes when using bulk materials. Plasmonics becomes a useful solution, harnessing greater intensities of light and providing large opportunity for control of light at the nanoscale. However, there still exists issues in plasmonics including, limited ranges of frequencies and property manipulations. This thesis addresses these issues by exploring mixtures of AuNPs and AgNCs to expand the accessible frequency ranges of plasmonics within a single system, thus allowing for greater manipulation of light. The main hypotheses of this thesis are: 
1) It is predicted that by mixing AuNPs and AgNCs, a system containing multiple plasmonic modes spanning greater ranges of the UV-vis spectrum will be obtained. i.e., more frequencies will be accessed.

2) These plasmonic modes were predicted to be tunable across the UV-vis-NIR spectrum as the local environment is manipulated by:

a. Deposition onto a thin polystyrene film

b. Embedment of NPs into the polystyrene film via conventional heating

c. Thermoplasmonic embedment of the individual AuNPs and AgNCs near their dipolar resonances (568 $\mathrm{nm}$ and $458 \mathrm{~nm}$ respectively)

3) Using mixtures of $\mathrm{Au}$ and $\mathrm{Ag}$ nanoparticles to probe a plasmonic gap mode between the NPs and a MS it was hypothesized that plasmonic colouring different from that observed in the individual NP system be achieved with greater tunability.

\section{Methods:}

\subsection{Chemicals}

Hydrogen tetrachloroaurate (III) hydrate $\left(\mathrm{HAuCl}_{4} \cdot \mathrm{H}_{2} \mathrm{O}, 99.8 \%\right)$ was purchased from Strem Chemicals. Tri-sodium citrate (citrate, $99-100 \%$ ) was obtained from BDH. Silver nitrate (AgNO3, 99+\%), polyvinylpyrrolidone (PVP, $\mathrm{Mw} \approx 55000$ ), anhydrous $99.8 \%$ ethylene glycol (EG), $\mathrm{Na}_{2} \mathrm{~S}$ and $\mathrm{NaCl}, \mathrm{CHCl}_{3}$ were purchased from Sigma-Aldrich and used as obtained. 1,2dioleoyl-sn-glycero-3-phosphocholine (powder, DOPC) was purchased from Avanti Polar Lipids as a powder and used as obtained

\subsection{Synthesis of AgNCs}

AgNCs were synthesized based on a modified approach to a method developed by Skrabalak et al. ${ }^{80}$ The cubes were made by combining $\sim 0.4 \mathrm{~g}$ of 55,000 MW polyvinylpyrrolidone (PVP) with $15 \mathrm{~mL}$ ethylene glycol anhydrous (EG) in a round bottom flask (with a PPTE coated 
stir bar) and heating the solution to $150{ }^{\circ} \mathrm{C}$ in a oil bath while stirring at $300 \mathrm{rpm}$. Once the solution had reached $150^{\circ} \mathrm{C}$, it was left to stir at $300 \mathrm{rpm}$ for one hour. After, a $0.5 \mathrm{M} \mathrm{NaCl}$ solution was prepared by dissolving $\sim 0.2808 \mathrm{~g}$ of $\mathrm{NaCl}$ in $10 \mathrm{~mL}$ EG in a glass scintillation vial and sonicating and vortexing until dissolved. A $3 \mathrm{mM} \mathrm{Na} 2 \mathrm{~S}$ solution was prepared by dissolving $\sim 0.0023 \mathrm{~g}$ of $\mathrm{Na}_{2} \mathrm{~S}$ (weighed out into a glass vial using a glass Pasteur pipette) in $10 \mathrm{~mL}$ EG. A $0.3 \mathrm{M}$ solution of $\mathrm{AgNO}_{3}$ was prepared by dissolving $\sim 0.48 \mathrm{~g}$ of $\mathrm{AgNO}_{3}$ anhydrous in $10 \mathrm{~mL}$ EG. The vials containing both the $\mathrm{Na}_{2} \mathrm{~S}$ and $\mathrm{AgNO}_{3}$ were covered in black electric tape as the reagents are photosensitive. When the PVP solution had been stirred for 1 hour at a steady temperature of 150 ${ }^{\circ} \mathrm{C}, 25 \mu \mathrm{L}$ of the $\mathrm{NaCl}$ solution was added followed by $175 \mu \mathrm{L}$ of the $\mathrm{Na}_{2} \mathrm{~S}$ solution. After these additions, the solution was allowed to stir for 5 minutes. Then, $1.5 \mathrm{~mL}$ of the $\mathrm{AgNO}_{3}$ solution was added at a rate of $0.1 \mathrm{~mL} / \mathrm{min}$ using a New Era Pump Systems automated syringe pump system. The reaction was continually monitored using a UV-Vis spectrometer by periodically taking small aliquots out of the reaction flask and dispersing in an ethanol-filled cuvette and taking the resulting $\mathrm{UV}-\mathrm{Vis}$ spectrum. Once the spectrum indicated the presence of a multipolar peak at $\sim 350 \mathrm{~nm}$ and a strong dipolar peak at $\sim 460 \mathrm{~nm}$ became visible in the UV-Vis spectrum, the reaction was removed from the heat and quenched in an ice bath.

\subsection{Synthesis of AuNPs}

This process uses a seed-mediated approach to size-controlled generation of gold nanoparticles. ${ }^{77}$ A $150 \mathrm{~mL}$ solution of $2.2 \mathrm{mM}$ trisodium citrate was added to a $250 \mathrm{~mL}$ round bottom flask and heated in an oil bath, stirring vigorously until boiling. Subsequently, a solution of $25 \mathrm{mM} \mathrm{HAuCl}_{4}$ and a solution of $60 \mathrm{mM}$ trisodium citrate was prepared. The use of a condenser reduced the evaporation of the solution. Once boiling was commenced, $1 \mathrm{~mL}$ of $25 \mathrm{mM} \mathrm{HAuCl}_{4}$ was injected, and the solution was left to stir for 10 mins. During this time, the solution changed from clear to a pink-red type colour. Once the seeds were synthesized, the solution was cooled to 
$90^{\circ} \mathrm{C}$. Following this, $1 \mathrm{~mL}$ of $25 \mathrm{mM} \mathrm{HAuCl}_{4}$ was added using a micropipette and left to stir for 30 mins. This injection was repeated twice more. After completion, $55 \mathrm{~mL}$ of the sample was extracted from the solution and $53 \mathrm{~mL}$ of deionized water was added to the reaction vessel. Then, $2 \mathrm{~mL}$ of $60 \mathrm{mM}$ citrate. This solution was used as a seed solution and the above process was repeated until the desired generation of nanoparticle was achieved. The reaction was monitored using UV-vis to evaluate the size, dispersity, and extinction maxima of the nanoparticles. If the sample was prepared over several days, then the $55 \mathrm{~mL}$ was still extracted at the end of the process, and the solution was quenched in an ice bath. The mixture was stored in the refrigerator overnight. The following day the solution was reheated to $90{ }^{\circ} \mathrm{C}$ and the above procedure was repeated until the desired generation was reached.

\subsection{Ligand transfer on AuNPs}

For usability purposes of the gold nanoparticles, the capping agent was transferred from citrate to poly(vinyl) pyrrolidone (PVP). Such transfer allowed for the deposition of the newly functionalized AuNPs onto a Langmuir-Blodgett trough. Previously synthesized AuNPs were separated into $2 \mathrm{~mL}$ centrifuge tubes until all the sample was consumed. The tubes were spun in a centrifuge at 10000 RPM for 10-20 mins. After centrifugation was completed, the supernatant was extracted leaving only the undisturbed gold nanoparticle pellet. Subsequently, the pellet was redispersed in $2 \mathrm{~mL}$ of $5 \mathrm{mM}$ PVP $(30 \mathrm{~g}, 150 \mathrm{~mL} \mathrm{H} 2 \mathrm{O})$, this was repeated for each of the respective centrifuge tubes. The newly redispersed pellets were then combined into a $250 \mathrm{~mL}$ Erlenmeyer flask containing a stir rod and left to spin on a Corning PC-420D magnetic stir plate at 300 RPM for 72 hours.

\subsection{Preparation of Polystyrene films on $\mathrm{SiO}_{2}$}

A $10 \mathrm{~mL}$ solution of $3 \mathrm{wt} \%$ polystyrene (PS) in toluene was prepared in a $20 \mathrm{~mL}$ scintillation vile containing a glass stir bar. This solution was spun for $3 \mathrm{hrs}$ at the maximum speed 
allowed by the stir plate. After the solution was completely dissolved it was filtered through a 0.22 $\mu \mathrm{m}$ polytetrafluoroethylene filter that had been previously rinsed with toluene. Concurrently, several glass microscopy slides $(30 \mathrm{~mm}$ x $30 \mathrm{~mm}$ x $1 \mathrm{~mm})$ were cut into 3 smaller slides using a diamond tipped pen and incubated in piranha solution for $10-20$ mins. Once complete, the slides were washed with deionized water to remove excess piranha. Each slide was individually sonicated in methanol (to maintain cleanliness) and dried with nitrogen. The slide was then placed securely onto a homemade spin coater. Using a Pasteur pipette, the PS was added to the attached glass slide ensuring that the slide was saturated with the solution and no air bubble were present. The slides were spun at approximately 4000 RPM for $30 \mathrm{sec}$ and positioned upright in a glass petri dish to evaporate off the toluene. All slides were left to dry in air for $1 \mathrm{hr}$, then placed in an oven overnight at $130^{\circ} \mathrm{C}$ with the lid propped slightly open using the large end of the Pasteur pipette. The next day, the oven was turned off and the slides were left to cool in the oven until room temperature was reached.

\subsection{Deposition of Nanoparticles onto Polymer-SiO ${ }_{2}$ Substrate}

The Langmuir-Blodgett trough was first cleaned using chloroform, then filled with deionized water. Deionized water was then aspirated off the surface of the trough then the water was replenished and left to sit for 20 - 25 minutes. An isotherm was run using a bar speed of 50 $\mathrm{mm}^{2} / \mathrm{min}$; a pressure greater than or equal to $0.2 \mathrm{mN} / \mathrm{m}$ in the isotherm indicated that the through required further cleaning to be repeated until the pressure remained below the limit. Enough nanoparticle solution was obtained to achieve a final extinction of approximately 200. The nanoparticle fraction was centrifuged for $10-20$ minutes at 10000 RPM and redispersed in the same volume of ethanol, repeating this step once more to ensure remaining PVP was washed off. The sample was centrifuged again for $10 \mathrm{~min}$ at $10000 \mathrm{RPM}$ and redispersed in $1 \mathrm{~mL}$ of $\mathrm{CHCl}_{3}$ and spun for 5 minutes at 8000 RPM. Once the supernatant was removed, excess solvent was 
evaporated using nitrogen. Using a micro syringe, $300 \mu \mathrm{L}$ of chloroform was added to the pellet then transferred into a $5 \mathrm{~mL}$ scintillation vile using a micro syringe. Subsequently, $10 \mu \mathrm{L}$ of ethanol and $5 \mu \mathrm{L}$ of $1 \mathrm{mg} / \mathrm{mL}$ DOPC (in ethanol) was added, and the solution was sonicated for 30 seconds. The nanoparticle solution was deposited dropwise onto the trough using a micro syringe and left to sit for 10 minutes. An isotherm was run to observe the phases of the monolayer and the through was annealed by running an isotherm cycle when needed. When the trough was prepared, the previously made slides were tapped gently onto the surface to transfer the monolayer from the trough to the slide, drying the slides under nitrogen to remove excess liquid. This was completed at various pressures of the monolayer, controlling the pressure via the movement of the barrier. Slides were later analyzed using UV-vis and atomic force microscopy (AFM).

\subsection{Instrumentation}

$\mathrm{UV}-\mathrm{V}$ is extinction spectra of the colloidal nanocubes in ethanol and of deposited nanoparticle monolayers were recorded using a Shimadzu UV-2450 UV-Vis spectrophotometer. Baseline spectra of the underlying substrate, such as the PS thin film, were subtracted off the extinction spectra of the monolayers. Transmission and reflection spectra of the substrates were obtained using a Cary 7000 equipped with a universal measurement analyzer. Topography measurements of the deposited monolayers were done using Ntegra (NTMDT) atomic force microscope in semi contact mode in air at $23^{\circ} \mathrm{C}$ with $512 \times 512$ points per image. A $100 \times 100 \mu \mathrm{m} 2$ scanner (Ntegra) and silicon cantilevers with a reflective gold coating were used $(1.75 \mathrm{~N} / \mathrm{m}$ spring constant, $70 \mathrm{kHz}$ resonant frequency, NSG03, NT-MDT). Transmission Electron Micrographs were collected on a FEI Tecnai G2 F20 TEM at the Carleton University Nano-imaging Facility.

\subsection{Determination of 1:1 extinction mixture of AuNPs and AgNCs}

Average concentration of the nanoparticles was determined using information extracted

from the obtained AFM data. Particle density $\left(\mathrm{NPs} / \mu \mathrm{m}^{2}\right)$ was acquired by counting the particles 
which occupied a selected area and averaged over several areas on the same image. With the given density information, the extinction cross-section could be obtained.

\subsection{Selective Embedment of the Nanoparticles into the Polymer Film}

\subsubsection{Description of Optical System}

The excitation experiments were done with a continuous wave (CW) $\mathrm{Ar} / \mathrm{Kr}$ laser (Coherent

70 series) through an inverted microscope (Olympus IX-71) with a 20X objective. The laser was set at varying power $(\mathrm{mW})$, current $(\mathrm{A})$ and wavelength for different experiments. Power was measured by a power meter located immediately before the focusing lens.

\subsubsection{Determining Embedment Threshold}

The first laser-exposure experiments were conducted to determine the embedment threshold of the nanoparticles. The wavelength of the laser was held constant and the current, power, and exposure time was varied. AFM images of the AgNC monolayers were taken before and after laser exposure to determine if embedment had occurred.

\subsubsection{Investigating Selective Embedment}

To perform the selective embedment experiments various wavelengths (458 nm and 568 $\mathrm{nm}$ ) and constant power (22 $\mathrm{mW}$ and $50 \mathrm{~mW}$ respectively) were used. The laser exposure time was varied. The samples were imaged by AFM before and after laser exposure. Embedment was examined by comparing the AFM images before and after laser exposure and using various features in the Nova software to measure the topographic height of select features before and after laser exposure.

\subsection{Fabrication of Metal thin films.}

The Carleton University Microfabrication Facility prepared $50 \mathrm{~nm}$ of Au or Ag films which were deposited onto glass slides pre-cleaned in piranha solution (1:5 H2O2:H2SO4) by thermal evaporation on a Balzers BA510 system. Au deposition was preceded by deposition of a $10 \mathrm{~nm} \mathrm{Cr}$ adhesion layer. 


\subsection{FDTD Simulations}

Finite Difference Domain modelling was performed using a Lumerical Solutions Inc software program (Lumerical Solutions v8.15). A total-field scattered-field light source was used to calculate the absorption cross sections. The parameters used for the simulation settings had been previously verified using convergence testing. The simulation time was set to $100 \mathrm{fs}$ and an 800 $\mathrm{nm}$ cubic simulation space was used. The models consisted of silver nanocubes with edge lengths of $60 \mathrm{~nm}$ with $15 \%$ corner roundness, and spherical gold nanoparticles of $30 \mathrm{~nm}$ radius, both having a $2 \mathrm{~nm}$ PVP capping layer, supported on a $150 \mathrm{~nm}$ thick PS film. The materials were described by an analytical model fitted to tabulated permittivity data of silver, gold, polystyrene and PVP. A mesh override region extending at least $10 \mathrm{~nm}$ past each nanoparticle or structure with a $0.5 \mathrm{~nm}$ mesh size was used for all simulations. X-axis and y-axis symmetry boundary conditions (symmetric and antisymmetric). The absorption simulations they were conducted from $300 \mathrm{~nm}$ $900 \mathrm{~nm}$ with a total of 301 points collected. Reflection and Transmission measurements were modelled using a plane wave source using a center span with the center wavelength and wavelength span set to $600 \mathrm{~nm}$.

\section{Results and Discussion:}

This projects aim was to explore the optical properties of mixtures of nanoparticles, namely silver nanocubes (AgNCs) and gold nanoparticles (AuNPs) when situated above a substrate. The nanoparticles were first individually deposited onto PS films. Understanding of both types of nanoparticles (i.e., AgNCs or AuNPs) and their optical properties as individuals on a substrate was necessary for designing mixtures of the NPs. Once the optical properties were understood for individual particles, the nanoparticle concentrations needed for a 1:1 mixture was determined. A 
1:1 mixture serves as a baseline for understanding the system as it was presumed that it would produce a 1:1 extinction.

These mixtures were first tested for their changes in optical properties in response to conventional heating. When the substrates are heated above the glass transition temperature of the polymer, the nanoparticles embed into the polymer; their environment is modified. Alteration of the local environment of nanoparticles lead to changes in their optical properties. Afterwards, spectrally selective thermoplasmonic embedment was utilized to observe if different types of nanoparticles (i.e., AgNCs or AuNPs) could be embedded.

Silver nanocubes and gold nanoparticles have different dipolar resonances (e.g., herein, 418 and $532 \mathrm{~nm}$ in the mixture). Excitation of the plasmons, especially at their LSPR (with sufficient power), leads to thermal dissipation to the surroundings, in this case polystyrene. As the nanoparticles transfer their heat to the polymer, it is heated sufficiently for congruent embedment of the nanoparticles, thus changing the local environment and optical response. Using different laser wavelengths, 458 and $568 \mathrm{~nm}$, it was expected that the individual AgNCs or individual AuNPs would embed. These wavelengths of light are in resonance with the dipolar plasmon mode and result in its excitation. Selecting to embed either AgNCs or AuNPs would result in changes in optical response of only the individual particles. This would allow for the fine manipulation of the optical properties of the nano systems via selective thermoplasmonic embedment.

The mixtures were then deposited on top of thin metal films (Ag or Au) coated with PS. Doing this allowed for the formation of plasmonic gap modes by coupling the NP LSPR to the MS SPs. A result of the plasmonic gap modes is the formations of strong, plasmon-induced colours which are very different from the in solution, or on a dielectric substrate colour. Moreover, as the nanoparticles are embedded into the polymer, and brought closer to the metal surface, the gap 
mode should experience a red shift and resonance intensity is expected to increase. Since the mixtures should produce multiple plasmon modes on PS, it was hypothesized that there would exist multiple gap plasmon modes when the mix is above a MS separated by PS. Colours different from the gap mode produced by individual particles over a MS should be achieved by using mixtures of AuNPs and AgNCs, and manipulation of their separation distance (alongside NP embedment into a polymer film) should produce an entirely different range of colours.

\subsection{Nanoparticle Synthesis}

\subsubsection{AgNC Synthesis}

Silver nanocubes were synthesized via the well-known polyol synthetic method; ${ }^{80}$ using said method, the particles were synthesized to the desired sized, monitoring their size (and shape) spectroscopically, advantageously, using their LSPR to do so. After only nine minutes, it was observed that the silver seeds had been generated as represented by the broad dipolar peak at a maximum of $404 \mathrm{~nm}$ (fig. 21). Overtime, the dipolar peak position was monitored and the appearance of the multipolar peak near $350 \mathrm{~nm}$ began after $17 \mathrm{mins}$ of synthesis. Once the multipolar peak was present, it acted as an indicator that there are, indeed, silver nanocubes formed. This set of AgNCs was quenched and subsequently centrifuged to remove unreacted reagents, as well as concentrate the nanoparticle solution. The final AgNCs solution produces spectrum with multiple plasmon modes. ${ }^{3,80}$ Moreover, the synthesis of AgNCs was confirmed using TEM (inset figure 21). 


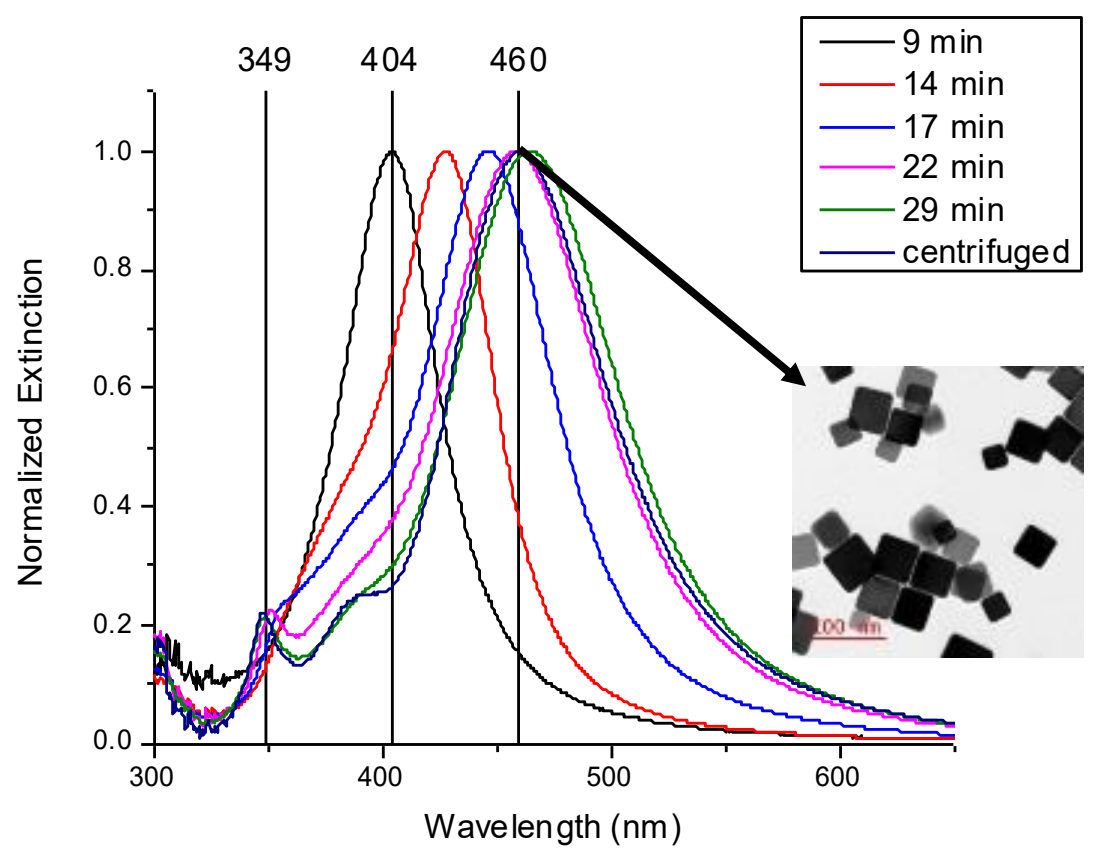

Figure 21: Monitoring the polyol synthesis using UV-vis. Inset TEM image of final AgNCs.

The AgNCs were then deposited onto prepared thin polystyrene (PS) films $(\sim 150 \mathrm{~nm}$ thick). This system has been extensively studied, ${ }^{16,109,133,134}$ and the ability of silver to support higher order plasmon modes can be observed in fig. 21. This nanoparticle over a dielectric is an excellent model of the hybridization experienced upon symmetry breaking. Using the LangmuirSchafer (LS) technique (a subsidiary of Langmuir-Blodgett (LB)), the nanoparticle monolayer is transferred to the surface of the polymer film by means of a vertical stamp of the polymer material onto the nanoparticle interface. From fig. $22 a$ it can be observed that, in solution the dipolar peak is strongly absorbing, but the quadrupolar and multipolar peaks are weak. After deposition upon a 
substrate, a relative increase in the absorption of the quadrupolar mode is provided. This phenomenon is presented well experimentally, as well as in the FDTD model (Fig. 22b).

a)

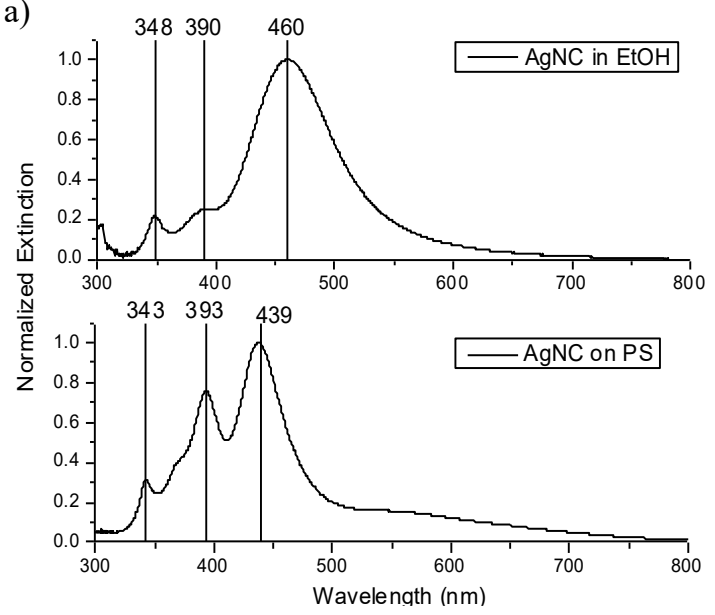

b)

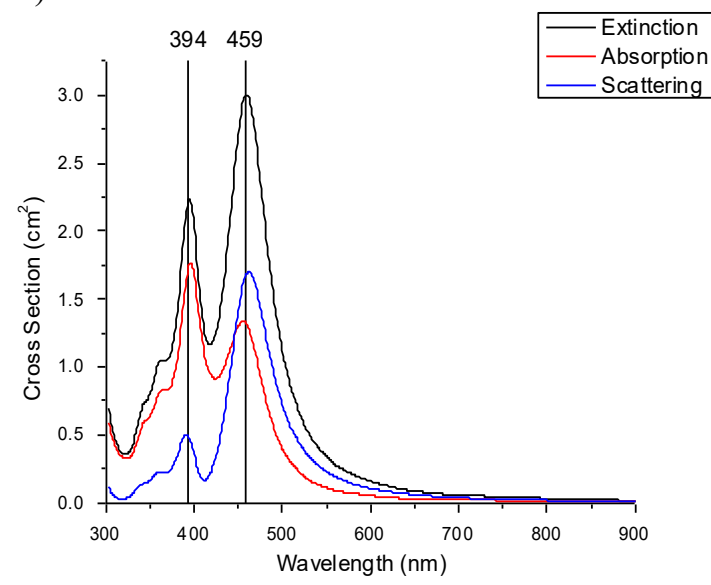

Figure 22: Extinction spectra of a) experimental AgNCs in solution (top) and on a PS substrate (bottom), and b) FDTD modeled scattering, absorption, and extinction cross-section of $60 \mathrm{~nm}$ AgNCs on PS.

After the NCs have been deposited there is a resultant change to the extinction spectrum. On the substrate, the dipolar (D) resonance becomes $439 \mathrm{~nm}$ compared to the lower energy 460 $\mathrm{nm}$ in solution. More importanly, the intensity of the quadrupolar (Q) mode drastically increases when atop a dielectric substrate; there is a percievable increase in the peak intensity. In this case, the peak shift is minute - changing from $390 \mathrm{~nm}$ to $393 \mathrm{~nm}$ in solution and on a substrate respectively. The multipolar (M) resonance was $348 \mathrm{~nm}$ in solution and blue shifted to $343 \mathrm{~nm}$ after deposition. Being that exinction is the sum of the absorption and scattering cross-sections, it is an optical property of concern for describing plasmonic systems. The FDTD model confirms that the experimental results are meaningful, providing an extinction profile with kindred spectral shape. For a system containing AgNCs with an edge length of $60 \mathrm{~nm}$, the FDTD model predicts that there will be a blue shift in the plasmonic responses of $\mathrm{D}$, and $\mathrm{Q}$ will undergo a slight blue shift along with an increase in intensity. As well, the narrowing of the dipolar peaks is predicted 
in the model and observed experimentally, indicating higher selectivity. In both experiment and in the model Q has an intensity that is $3 / 4 \mathrm{D}$. Hence, AgNCs can be deposited onto PS thin films to produce the predicted hybrib plasmonic system presented.

Further, these materials can be observed for their abilty to transmit or reflect light. These phenomena are related to the colours that the the nanomaterials exhibit. Using instruments with higher sensitivity, which measure transmission or reflection, can provide more information on the materials optical properties. More specifically, when observing more complex nanoassemblies like a NP over a mirror (NPoM) system - extinction becomes negligible due to high surface reflectivity. This means that incident light will not transmit readily through these materials. Hence, observing the transmission and reflectance spectra becomes an asset in more complex nanoassemblies. Section 3.4 of this thesis observes multiple NPoM systems using reflectance measurements for spectral determination.

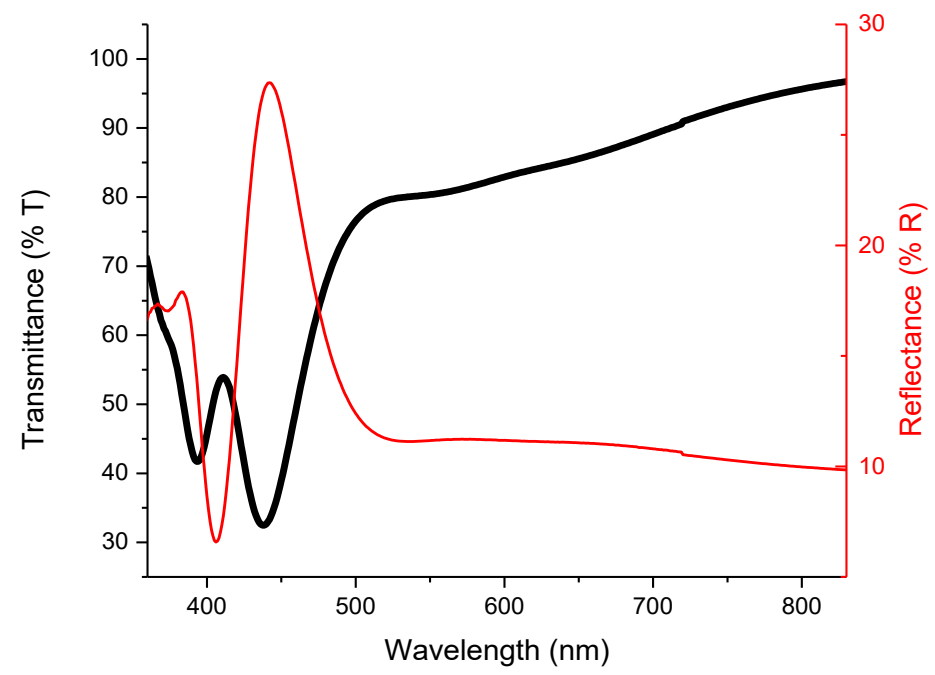

Figure 23: Respective transmittance and Reflectance spectra for AgNCs on PS

The transmission and reflectance spectra of AgNCs, shown in fig. 23, idenitfy further the optical response of AgNCs supported by a PS film. Observing first the simpler system, of AgNCs 
provides a basis for describing what occurs in the NPoM system. For AgNCs on PS, the transmittance of light through the material is low at both the D and Q modes indicating that there is strong absorbance of light at these wavelengths. Contrastingly, the reflectance is very sharp near the dipolar wavelength. Otherwise, these materials transmit light at remaining wavelengths (i.e. this substrate is not highly reflective). As will be observed in section 3.4 , the reflectance of the substrates is responsible for the observed colour. The reflectance spectrum has a peak that is near the dipolar resonance observed in the extinction spectrum for the AgNCs on PS (fig. 23), so the reflectance of the substrate is most intense at the D mode. As well, the transmission spectrum shown in fig. 22 is the inverse of the observed extinction spectrum (fig. 22); they essentially have the same meaning with the caveat being one is based on determining how much light is transmitted vs how much is absorbed (and scattered). Preforming these measurements provide a basis for the optical properties exhibited by AgNCs supported by PS.

\subsubsection{AuNP Assembly}

\subsubsection{AuNP Synthesis}

Firstly, the Au nanoparticles were synthesized using a previously reported seed-mediated method using tri-sodium citrate as the reducing and the capping agent. ${ }^{77}$ Unfortunately, Citrate does not have compatible intermolecular forces for binding to polystyrene nor is it favourable for Langmuir films, as it is a hydrophilic molecule, but poly-vinyl pyrrolidone (PVP) has been used for both. ${ }^{109}$ A sample that had an extinction (e) sufficiently high enough to deposit the particles onto the Langmuir-Blodgett trough and provide a consistent monolayer with visible colouring was desired. If the extinction is too high, then the particles may begin to form extremely dense monolayers, and even clusters. Too low and there may not be enough particles to form a consistent monolayer, so colouring would be lowered. 
Using the seed mediated approach, nanoparticle solutions of different sizes (and concentration) were synthesized, and their corresponding spectra are shown in fig. 24a. The initial seed solution (G0) presented a spectrum with an LSPR wavelength of $510 \mathrm{~nm}$. Nanoparticle growth continues onward producing larger sized nanoparticles in each generation, and hence changes to the plasmonic response. The seed mediated approach is beneficial as it reduces the probability of Oswald's ripening by selecting and controlling seed growth and concentration. ${ }^{77}$ Generation 10 was the final AuNP solution selected here for its specific LSPR of $553 \mathrm{~nm}\left(\mathrm{D}_{\mathrm{Au}}\right)$ which offered potential for exceptional spatial and spectral differences from $\mathrm{AgNCs}\left(\mathrm{D}_{\mathrm{Ag}}\right.$ on PS reported herein as $439 \mathrm{~nm})$.

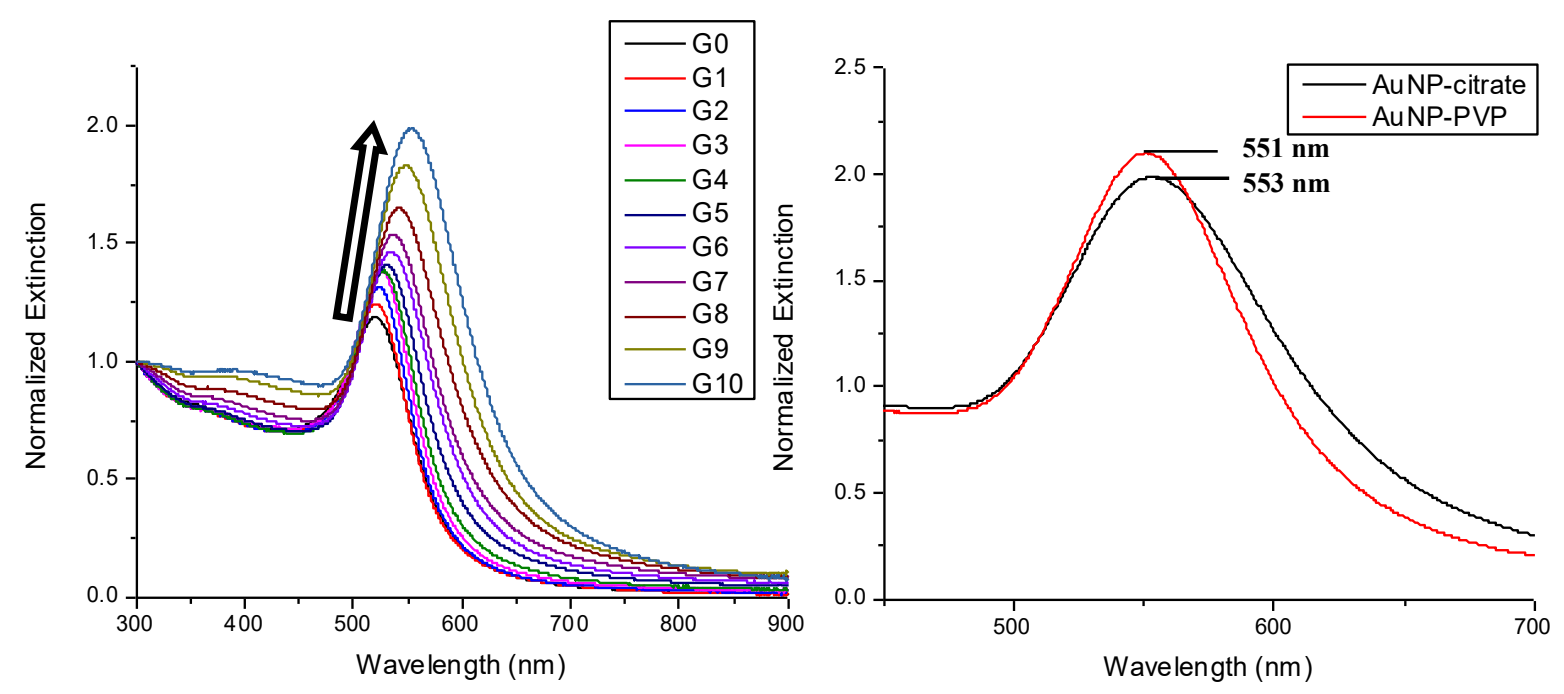

Figure 24: Extinction spectra for the AuNP synthesis. AuNP growth from seed (G0) to final AuNPs (G10) with average particle size of $68 \mathrm{~nm}$ (left) and ligand transfer from citrate to PVP (right).

Afterwards, the particles from G10 underwent ligand transfer from citrate to PVP and were concentrated to a smaller volume via centrifugation, and hence a higher extinction NP solution could be yielded. It should also be noted that the ligand transfer can be confirmed spectrally, as different surfactants have different impacts on the refractive index. Here, a blue shift of $2 \mathrm{~nm}$ was observed confirming that the ligand transfer was successful (fig. 24b). 


\subsubsection{Langmuir Schafer deposition of AuNPs on PS}

Fig. $24 a$ is the extinction spectrum for a 100X dilution of AuNP-PVP. The final extinction

value obtained for this synthesis of AuNPs was 127.5, sufficient to deposit the nanoparticles onto the Langmuir trough and yield a consistent monolayer of reasonably high particle density.

a)

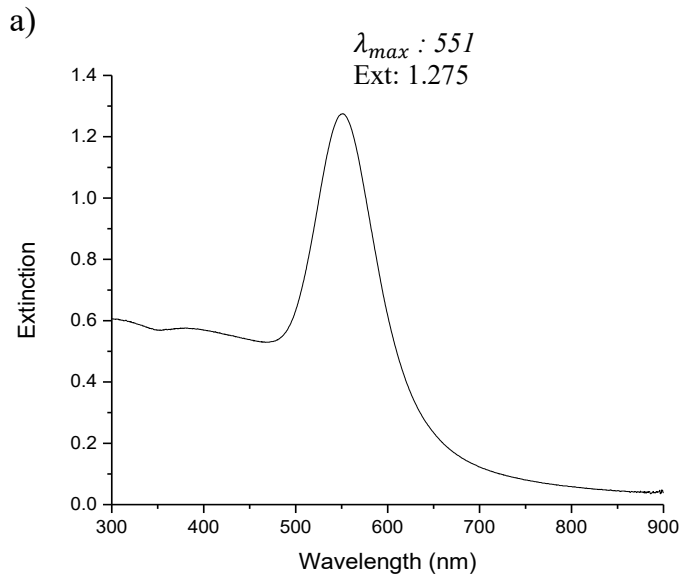

c)

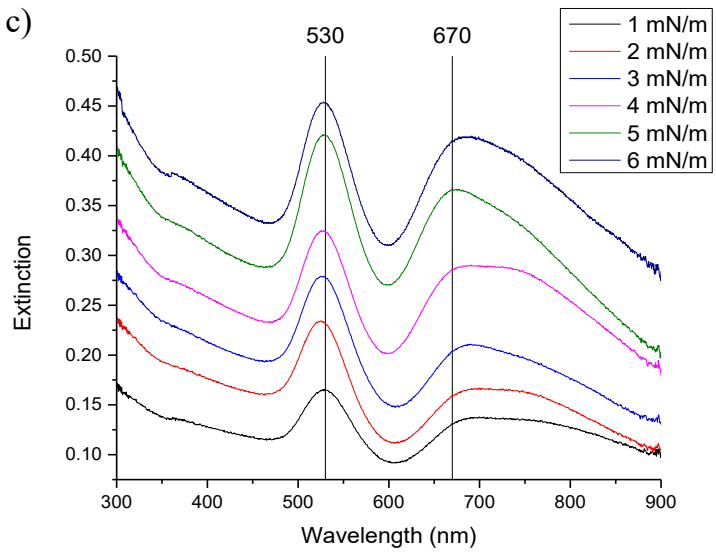

b)

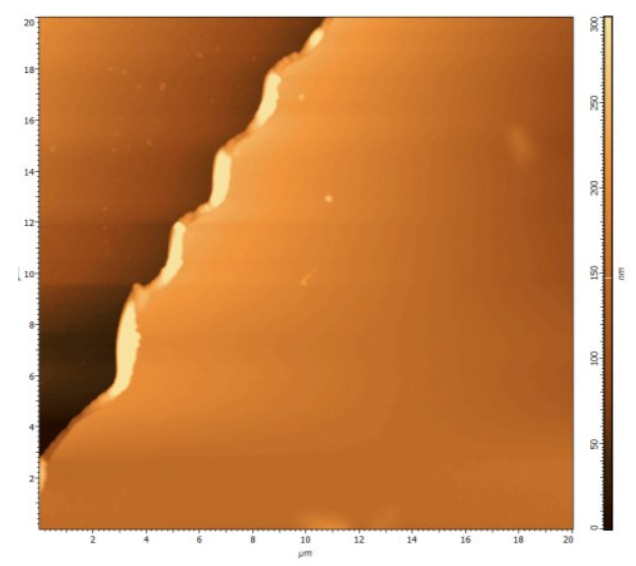

d)

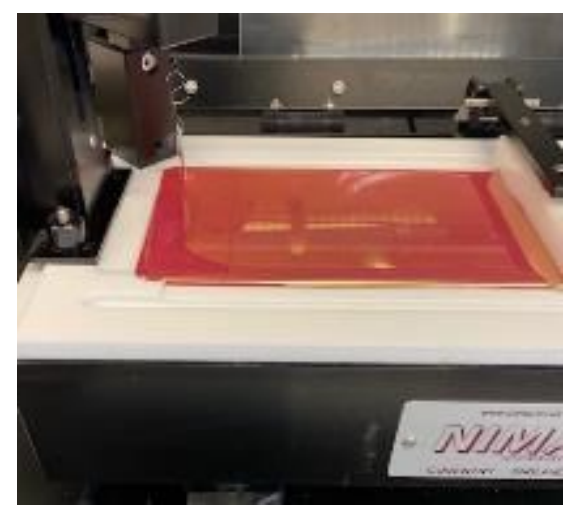

Figure 25: Experiments of Au NPs. a) final AuNP-PVP extinction spectrum 100X in EtOH, b) AFM image of a smooth PS film measuring $150 \mathrm{~nm}$ in height, c) spectrum of AuNPs-PVP on PS at different surface pressures, and d) Langmuir trough with AuNPs-PVP

Obtaining such a relatively high extinction allowed for ease of deposition of the nanoparticles onto the LB trough. The prepared polystyrene slides were approximately $150 \mathrm{~nm}$ thin, falling within the nano-regime; the height of PS was measured using AFM and the image is shown in fig. 54b. From the AFM image it can be observed that the polystyrene films were smooth and contained minimal debris (which could contaminate the sample). Deposition of the AuNPs 
onto the trough led to a bright red coloured monolayer (fig. $25 d$ ). Over time, it was observed that the nanoparticle monolayer had changed in colour, becoming a purplish-grey colour. Purple and grey are well associated with NP aggregation, as purple specifically is indicative of a larger particle, and its spectral position is commonly associated with nanoparticle dipole-dipole coupling. ${ }^{9} 135-137$

A variety of substrates at different surface pressures (ranging from 1-6 mN/m) were deposited and characterized. UV-vis spectra (fig. 25c) of the prepared substrates suggest narrow size distribution of the particles, but also that D-D coupling had occurred. At low pressure, 1 $\mathrm{mN} / \mathrm{m}$, a reasonably sharp peak is obtained $(525 \mathrm{~nm}, 0.162)$, with a broad coupling peak adjacent $(750 \mathrm{~nm}, 0.131)$. The intensity of the coupling peak, fig. $25 c$, was not significantly lower than that of the dipolar peak itself, indicating that there was likely a large amount of coupling, surely more than desired. Notably, as the pressure was increased consequent increases in the absorbance was observed for both the D and D-D modes (fig. 25c). Coupling could also be observed upon the slides themselves, taking on the noted colour that could be observed on the trough later in the process (purple-greyish colour). AFM imaging concluded the perceived coupling to be true, showing both well-spaced individual AuNPs, as well as clusters of various sizes, fig. 26.

Since the AgNCs on PS system has been well-explored, the particle densities have already been presented, ${ }^{18}$ and used herein for determination of the extinction coefficient and concentration, thus the AFM image was not included for AgNCs. Herein, examination of PVP capped AuNPs on PS was required for acquisition of particle densities, hence their AFM images and detailed calculations have been presented. 
a)

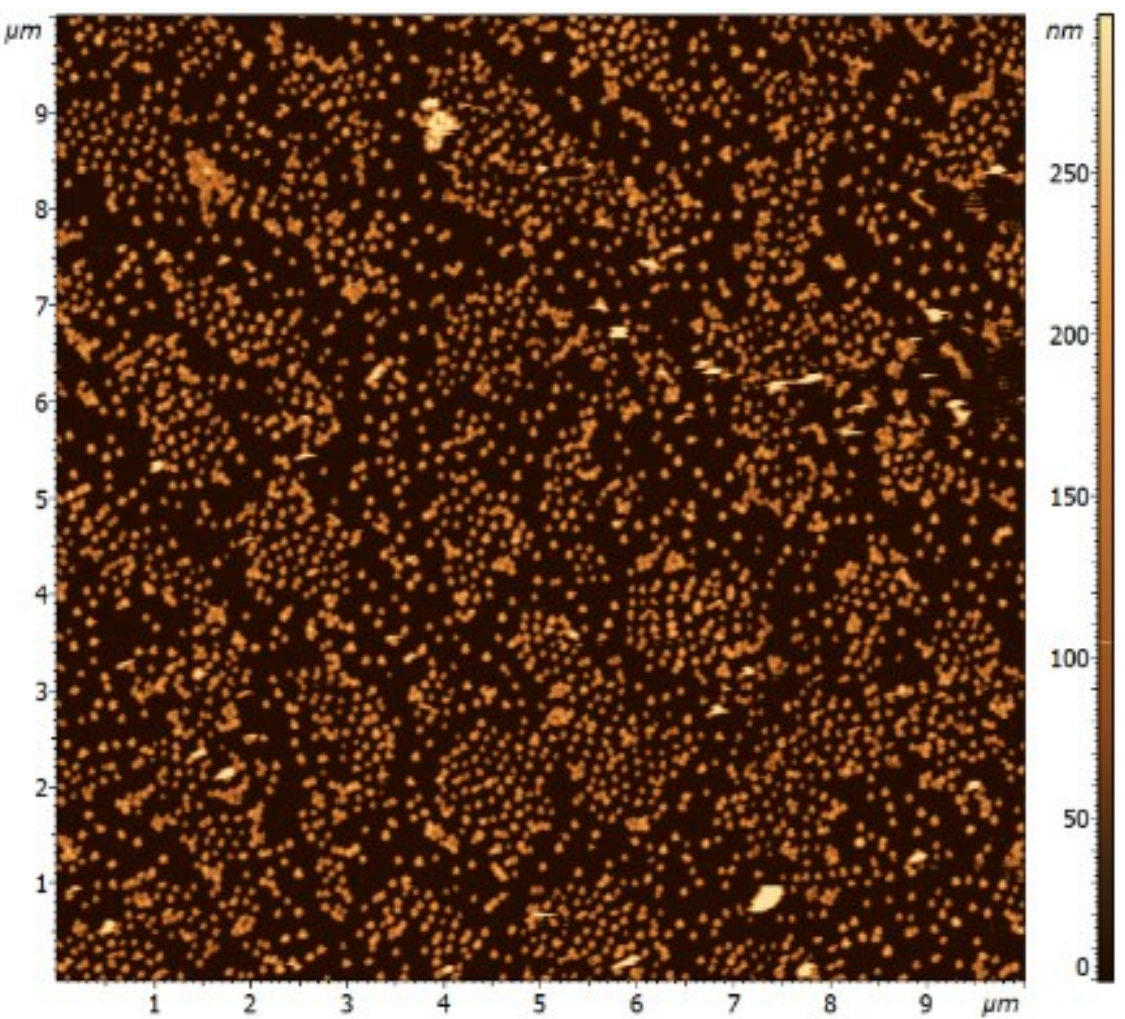

b)

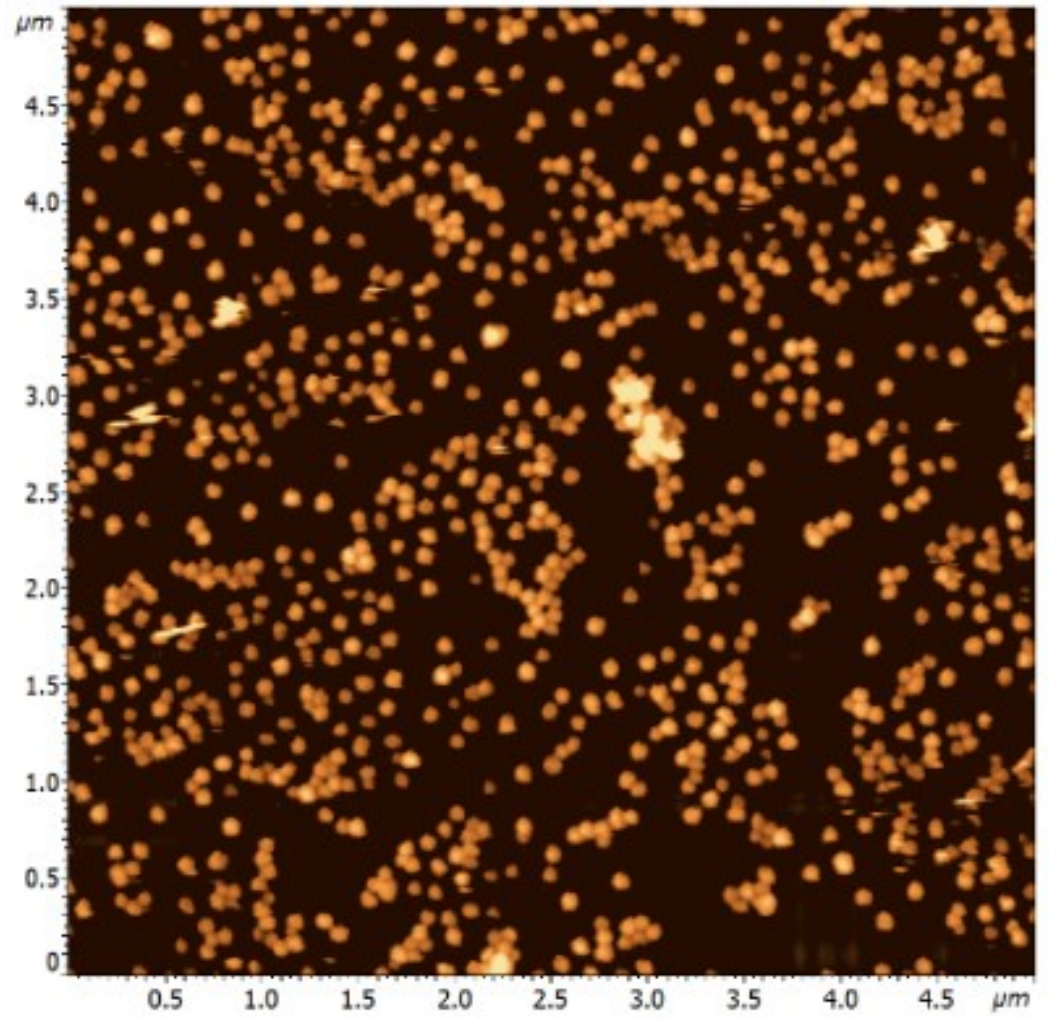

$n m$
$180+$
160
140
100
$80+$
$60+$
40
20

Figure 26: AFM images of $1 \mathrm{mN} / \mathrm{m}$ AuNPs on PS. a) $10 \times 10 \mu \mathrm{m}$ and b) $5 \times 5 \mu \mathrm{m}$. 
From the AFM image of AuNPs on PS, fig. 26, it was observed that there were both individual AuNPs, but also clustered nanoparticles. The individual particles are well-spaced, above the separation distance which would be required for a D-D coupling peak. Various types of clusters such as dimers, trimers, and larger aggregates can be observed from fig. 26 and produce significant plasmonic response.

Substrates with coupling peaks that had absorbance weaker than the dipolar resonance absorbance (1-3 mN/m samples, fig. 25c) were selected for presumed lower quantity of coupled nanoparticles. The AFM images in fig. 26 are for the $1 \mathrm{mN} / \mathrm{m}$ sample, (the 2 and $3 \mathrm{mN} / \mathrm{m}$ sample AFM images are presented in Section 6, fig. 49 and 50). An average particle density of 41 $\mathrm{NPs} / \mu \mathrm{m}^{2}$, determined by counting the number of NPs in the area, was calculated for the $1 \mathrm{mN} / \mathrm{m}$ substrate as seen in figure 26. A height profile of the sample provided an average particle size 63.1 $\mathrm{nm}$. Analysis of the UV-vis spectrum of the $1 \mathrm{mN} / \mathrm{m}$ slide provided an absorbance value of 0.161 , from this the extinction coefficient $\left(\epsilon, \mu \mathrm{m}^{2} / \mathrm{NP}\right)$ could be calculated using the following relationship:

$$
\begin{gathered}
I=I_{0} 10^{-\epsilon \sigma} \Rightarrow \frac{I}{I_{0}}=10^{-\epsilon \sigma} \\
\log _{10}\left(\frac{I}{I_{0}}\right)=-\epsilon \sigma \\
A=-\log _{10}\left(\frac{I}{I_{0}}\right) \\
\epsilon=\frac{A}{\sigma}
\end{gathered}
$$

where $\mathrm{I}$ and $\mathrm{I}_{0}$ are the intensity of light transmitted by the sample and the intensity of light irradiated onto the sample respectively, $\sigma$ is the nanoparticle surface density $\left(\mathrm{NPs} / \mu \mathrm{m}^{2}\right)$ in each monolayer, 
and $\mathrm{A}$ is the measured absorbance of the slide. ${ }^{18}$ From this, the extinction coefficient for the 1 $\mathrm{mN} / \mathrm{m}$ slide was $3.9 \times 10^{-3} \mu \mathrm{m}^{2} / \mathrm{NP}$. Moreover, from Beer's law it is also known that: ${ }^{138}$

$$
A=\epsilon c l
$$

hence, when the extinction coefficient is known, the nanoparticle concentration can be calculated for similar systems. With concentration of nanoparticle solutions being obtained, the possibility for more control of the deposition can be yielded. Specifically, various concentrations can be selected for the deposition of these nanoparticles at different densities. This determination was necessary for designing a system containing mixtures of $\mathrm{Au}$ and $\mathrm{Ag}$ nanoparticles at different ratios. The 2 and $3 \mathrm{mN} / \mathrm{m}$ slides were also processed for their extinction coefficient, for more accurate calculation of the extinction observed when the AuNP solution is deposited on the Langmuir trough. For both, the 2 and $3 \mathrm{mN} / \mathrm{m}$ slide $\epsilon$ was determined to be $4.3 \times 10^{-3} \mu \mathrm{m}^{2} / \mathrm{NP}$. The average of these three values was $(4.2 \pm 0.3) \times 10^{-3} \mu \mathrm{m}^{2} / \mathrm{NP}$ and this value was used for designing the new nanoarchitecture containing AuNPs and AgNCs.

\subsubsection{Conventional Heating of AuNPs on PS}

Nanoparticles are sensitive to changes in their local environment, and by modifying that environment there are corresponding spectral shifts, observable as a change in colour. By heating the substrate above the $\mathrm{T}_{\mathrm{g}}$ of the polymer film, the nanoparticles begin to embed, changing their local environment. As a result of NPs embedding into the polymer, the LSPR shifts, and a different colour is yielded. A FDTD model for AuNPs embedding into a PS film was conducted to mimic the result of heating the material above the polymer's $T_{\mathrm{g}}$. The model can describe the distinct spectral changes that are expected as the nanoparticle embeds. As the embedment depth increases, a sharp increase in extinction is expected. The model also informs the appearance of secondary modes with increasing embedment depth. 
Figure 27 shows the FDTD model for AuNPs (radius $=30 \mathrm{~nm}$, and $2 \mathrm{~nm}$ PVP overcoating) embedding into a $150 \mathrm{~nm}$ PS film. As the AuNPs embed into the PS, the LSPR strength increases; the peak intensity also continually increases. Moreover, an observable red shift can be noticed spectrally upon AuNP embedment. This follows suit with the expected result, since as the local environment was changed there were observed peaks shifts. Corresponding peak shifts in the model signify that colour change can be expected as these AuNPs embed in PS.
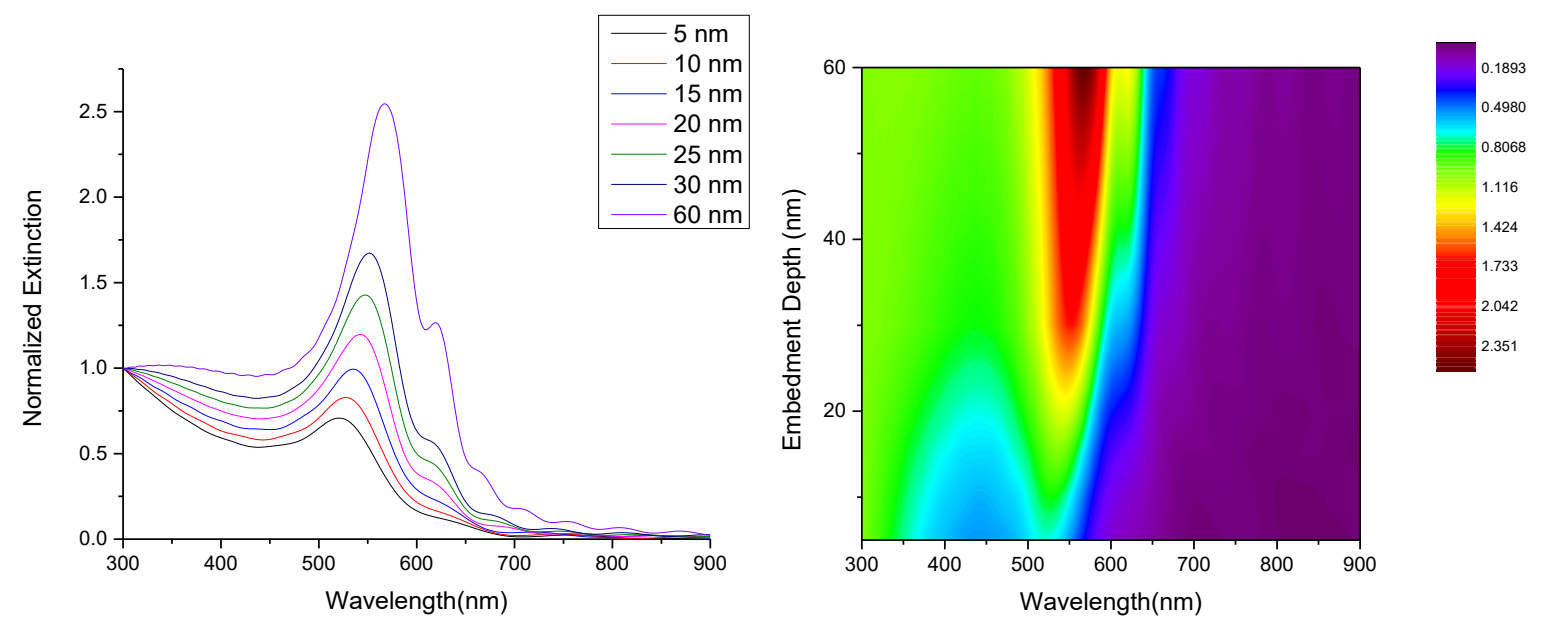

Figure 27: FDTD model of extinction for $60 \mathrm{~nm}$ AuNPs embedding into PS over a depth interval of 5-60 nm.

The experimental results for the deposition of AuNPs onto PS were also proceeded ( $f i g$ 28). After the initial deposition, the substrate was heated for 4,6 and 20 min. With 4 minutes of heating the nanoparticles LSPR red shifts from $523 \mathrm{~nm}$ to $555 \mathrm{~nm}$, there was a drastic increase in the peak intensity, and the particles had also embedded $36.6 \mathrm{~nm}$ from their initial height $(63.1 \mathrm{~nm}$, fig. 28c). Heating of the substrate for 6 , and then 20 minutes did not result in major changes to the plasmonic response - each shifting only $2 \mathrm{~nm}$ from the prior without large increases in peak intensity (fig. 28a). Remarkably, even after 20 minutes of heating, the AuNPs do not completely embed fully into the polymer, retaining an average height of $17.6 \mathrm{~nm}$ measured from the AFM scan (fig. 28d). Experimentally, fig. 28b, the trend was in line with that received in the FDTD 
model (fig 27 (right)) confirming that the nanoparticles embedded into the polymer and there were resultant changes in their plasmonic response.

The extinction spectra were not the exact same in the model, and in the experimental system (which bypassed some of the first embedment depths and included a coupling peak effecting resultant changes). Though they're not the same, the trend is very consistent from spectrum to spectrum showing red shifting plasmonic resonance with increasing intensity over time. As well, in experiment, there were no secondary modes picked up by the spectrometer, moreover, the model does not depict nanoparticle D-D coupling. Earlier in this section, it was noted that the experimental result contained aggregates (dimers, trimers, multimers) shown in the initial spectrum in fig. $28 a$ adjacent to the dipolar resonance, thus the corresponding spectrum is different from the model (fig. 27 (left)). Also observable in fig. $28 a$ the D-D coupling peak intensity diminished in absorbance with heating. So, the coupling resonance appears to become negligible as the substrate is heated. 
a)

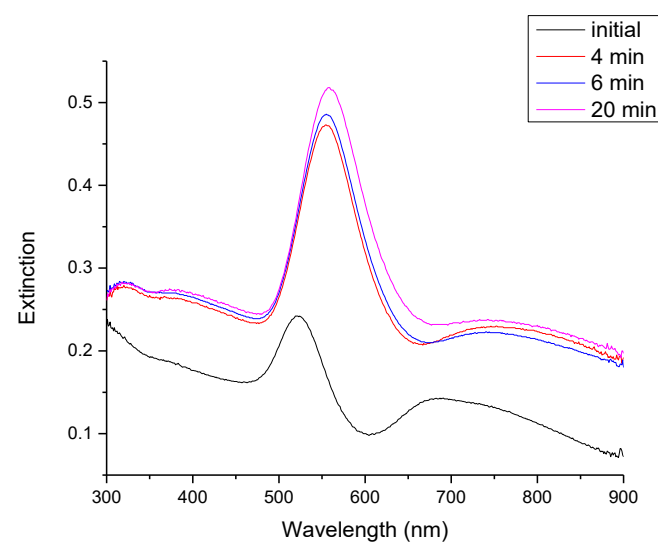

c)

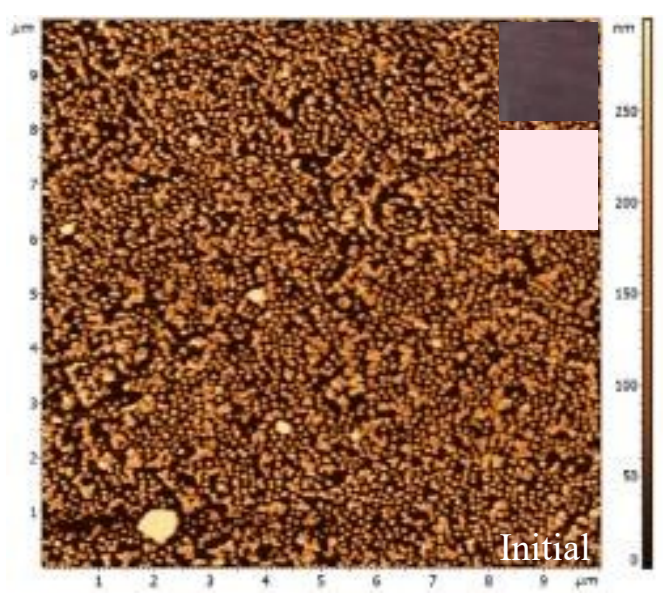

b)

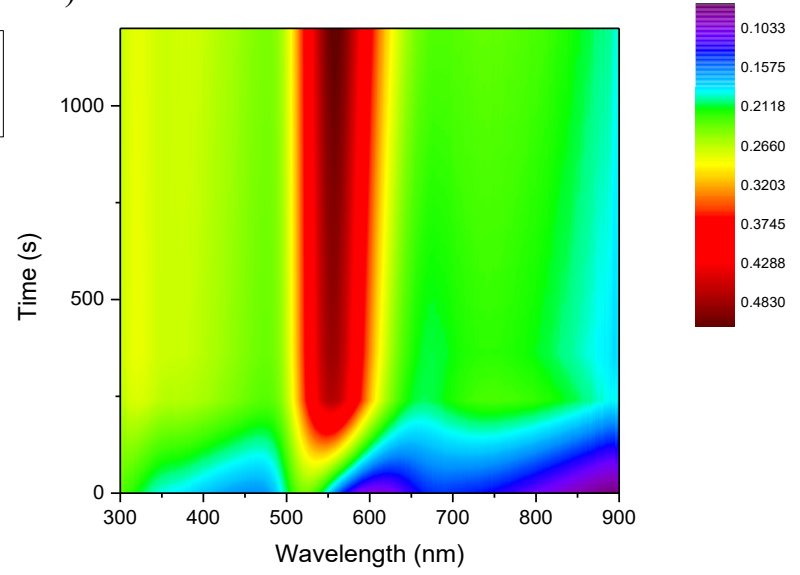

d)

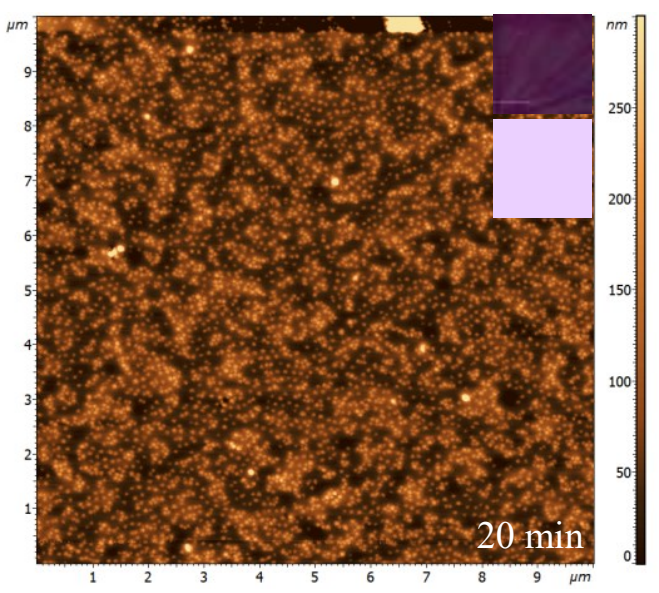

Figure 28: Experimental results of extinction for AuNPs embedding into PS over a time interval of 0-20 min at $\sim 125{ }^{\circ} \mathrm{C}$. Extinction spectra $(\mathrm{a}, \mathrm{b})$ and AFM images $(\mathrm{c}, \mathrm{d})$ of initial and final states; inset with the colour images in real-time and their colour representation based on CIE 1931 using their reflectance spectra.

The AFM image for the 20 min sample shows that the nanoparticles did embed with the polymer maneuvering itself around the embedding NPs and NP aggregates (appears as hazy material around NPs, figure 28d). Another important consideration is that there were notable changes in the transmittance (decreases), and reflectance (increases), shown in Section 6 fig. 51, both undergoing a spectral red shift. This was perceived as a colour change in the material as the NPs were embedded into the polymer. The actual experimental result for the colour of the slide before and after heating, as well as their CIE represented colours are presented inset fig. $28 \mathrm{c}$, and d. The experimental result initially has a grey-ish purple/pink colour, representing both the 
individual NPs (pink) and the coupled particles (purple/grey). After heating, the slide had a more consistent purple colour representative of the nanoparticles which have embedded into the polymer. The colour in the final sample is obtained from the reflectance of the Au dipole $\left(D_{A u}\right)$ with LSPR at $559 \mathrm{~nm}$. The CIE representation shows the pink and purple for the initial and final sample, with the colours being slightly muted as colour saturation (measure of colour intensity) was not accounted for in these representations.

In fig. 29 the comparison between the theoretical and experimental results for AuNPs embedding into PS has been presented. Firstly, the peak shift was compared to the relative LSPR position (fig. 29a) to show how the LSPR shifts. From the FDTD model, it was observed that the LSPR peak shift occurs rather linearly for AuNPs embedding into PS; the plasmonic response shifts linearly. The experimental embedment resulted in a similar trend to the model with a linear slope that approached the predicted result. This suggested that the experimental result was very similar to those observed in the model. After, the LSPR peak position was plotted (fig. 29b) as a function of the NP height which was determined using AFM topography measurements (refer to appendix), identifying the trend in the LSPR shift that occurred as the nanoparticles embedded into the polymer. The gradual slowing in peak shift was observed as the NP sunk lower into the polymer (fig. 29b); as the nanoparticles embeds more than $40 \mathrm{~nm}$ into PS, the change in the plasmonic response becomes miniscule - this trend is observable in both model and experiment. Hence, the model was a very accurate depiction of the results, and the results are powerful enough to provide great understanding of AuNPs atop a PS thin film and subsequent embedment of the nanoparticles into the polymer. 
a)

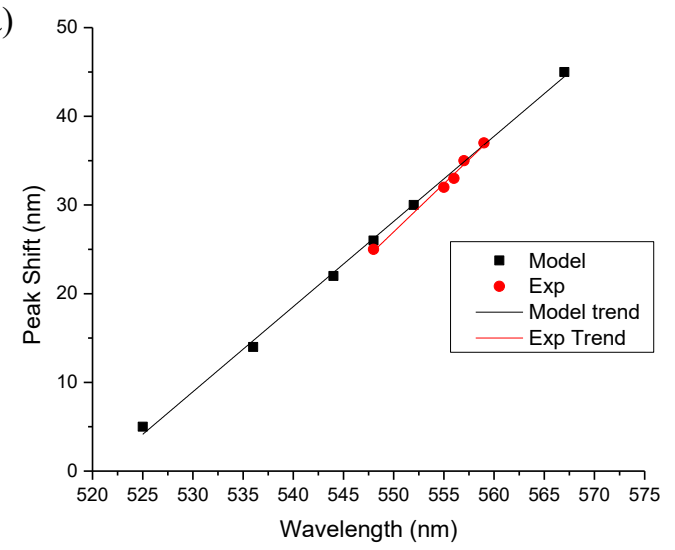

b)

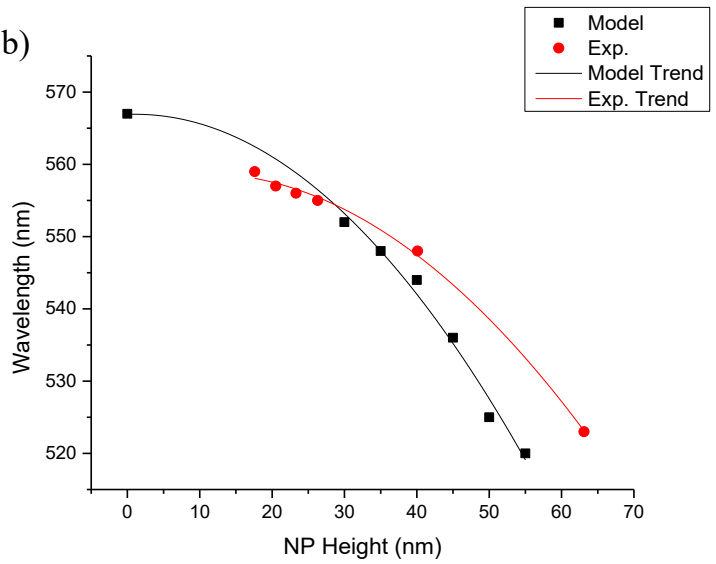

Figure 29: Comparison of FDTD Model and Exp Results of AuNPs embedding into PS over a depth interval of 5-40 $\mathrm{nm}$.

\subsection{Mixtures of Nanoparticles}

It has been observed that when AgNCs or AuNPs are deposited onto PS thin films, there results a corresponding change to their plasmonic response. AgNCs give distinct hybrid plasmon modes when deposited onto a dielectric surface (e.g., PS with $\left.\varepsilon_{r}: 2.4-2.7\right)$. AuNPs exhibit a blueshift when deposited onto polystyrene, giving a higher energy resonance than the in-solution NPs. Given all the knowledge gained from depositions of AgNCs and AuNPs onto PS films, the design of mixtures of nanoparticles could be proceeded utilizing their extinction as a prime optical property for accurate comparison and deposition. From this, it was expected that a simple plasmonic system containing multiple spectrally resolved plasmon modes across the visible spectrum be obtained. Using a 1:1 mixture of AgNCs and AuNPs was expected to provide a extinction spectrum with the narrow hybrid plasmon modes for $\mathrm{Ag}(\mathrm{D}, \mathrm{Q}, \mathrm{M})$ and $\mathrm{D}$ mode for $\mathrm{Au}$. It was also assumed that these plasmon modes would be tunable across the UV-vis-NIR spectrum via changes in the local dielectric environment, specifically by embedding the nanoparticles.

\subsubsection{Determination of concentrations for 1:1 extinction mixture of AuNPs:AgNCs}

The initial intent was to create a basis for the system by creating a 1:1 extinction mixture of AuNPs:AgNCs. The 1:1 mixture was prepared based on the relative extinctions of the nanoparticle solution. Choosing a 1:1 mixture gives a point of reference for referral when designing mixtures 
of different ratio. Using the NP extinction coefficients, the concentration of nanoparticles needed to acquire a final extinction of 100 for each NP solution was calculated (table 1). Once desired concentrations were yielded, the nanoparticle mixture could be generated. The spectrum of the AgNCs, AuNPs, and 1:1 mix are presented in fig. 30a, and fig. 30c shows the image of a 100X dilution of AuNPs, the mix and AgNCs respectively.

Table 1: Calculated values for determination of 1:1 mix of AuNP:AgNC

\begin{tabular}{|c|c|c|c|c|}
\hline $\mathbf{N P}$ & $\begin{array}{c}\boldsymbol{\epsilon} \\
\left(\mathbf{c m}^{2} / \mathbf{N P}\right)\end{array}$ & $\begin{array}{c}\text { Concentration } \\
\left(\mathbf{N P} / \mathbf{c m}^{\mathbf{3}}\right)\end{array}$ & $\begin{array}{c}\text { Desired } \\
\mathbf{A}\end{array}$ & $\begin{array}{c}\text { Desired } \\
\text { Concentration } \\
\left(\mathbf{N P} / \mathbf{c m}^{\mathbf{3}}\right)\end{array}$ \\
\hline $\mathrm{AuNP}$ & $4.17 \times 10^{-11}$ & $4.81 \times 10^{12}$ & 0.100 & $2.81 \times 10^{12}$ \\
\hline $\mathrm{AgNC}$ & $7.69 \times 10^{-11}$ & $7.62 \times 10^{12}$ & 0.100 & $1.30 \times 10^{12}$ \\
\hline
\end{tabular}

Referring to fig. $30 a$ it can be observed that both AuNPs and AgNCs in ethanol (EtOH) have spectra which match their characteristic shape; their spectra are identical to that observed for the typical in-solution particles. Once the NP solutions are mixed the spectrum underwent remarkable modifications. Importantly, Au appears to retain its spectral shape and position $\left(\mathrm{D}_{\mathrm{Au}}=\right.$ $553 \mathrm{~nm}$ ) in the mixture, but Ag presented a spectral blue shift (459 to $440 \mathrm{~nm}$ ), and the shape was distorted (mixing of $\mathrm{M}_{\mathrm{Ag}}$ and $\mathrm{Q}_{\mathrm{Ag}}$ ). The 1:1 mix spectrum also suggests that silver may have lost its features, specifically $\mathrm{Q}$ and $\mathrm{M}$, upon mixing with $\mathrm{Au}$. With the $\mathrm{Q}$ and $\mathrm{M}$ modes gone, it would be expected that the cubes would have undergone rounding of their edges, which offers explanation to the blue shift in $\mathrm{D}_{\mathrm{Ag}}$ due to changes in NP shape. Experimentation was continued nonetheless, and the deposited 1:1 mix on the trough had colour resembling the colour of the nanoparticles in solution. Over time the monolayer on the trough underwent changes in colour from the brownishyellow, pink colour (fig. 30d) to a more grey/red colour. The grey/red colouring indicated that nanoparticle coupling may have occurred. 
a)

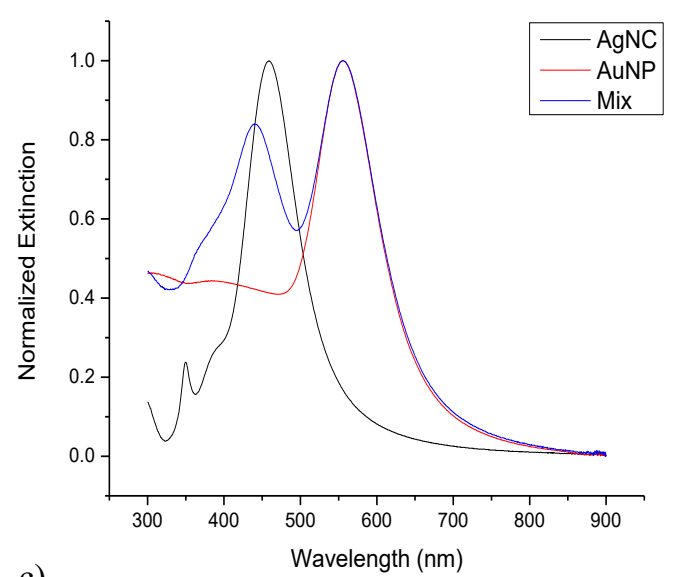

c)

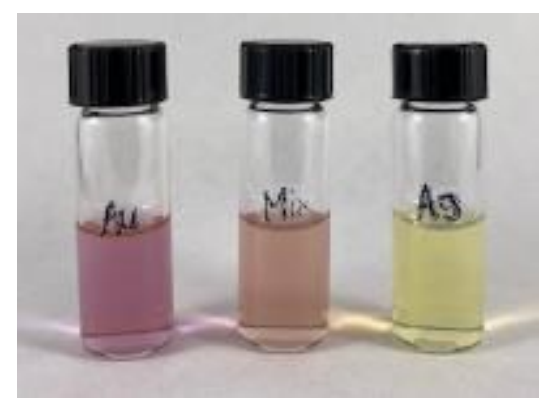

b)

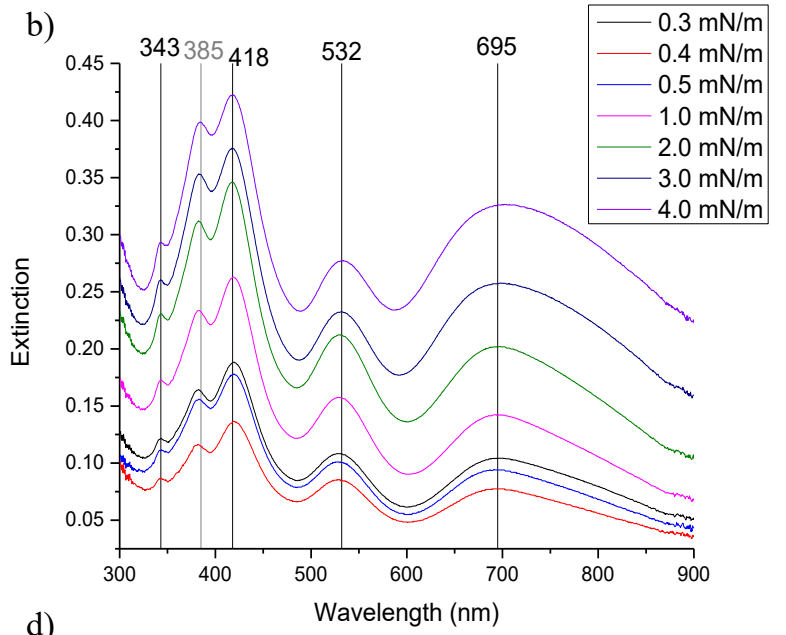

d)

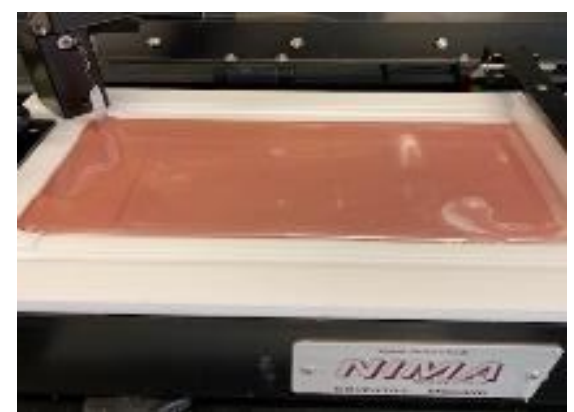

Figure 30: Experiments of 1:1 extinction (e) Mixtures of Au:Ag NPs. a) 100X dilution extinction spectrum of AuNPs, AgNCs and a 1:1 Mix, b) spectrum of 1:1 Mix deposited on $150 \mathrm{~nm}$ PS at increasing surface pressures, c) photograph of 100e NPs after 100X dilution, and d) Langmuir trough with 1:1 Mix.

Transferring of the nanoparticle monolayer was proceeded via LS at surface pressures from $0.4 \mathrm{mN} / \mathrm{m}$ to $4 \mathrm{mN} / \mathrm{m}$ and as a result the characteristic spectra for these substrates were obtained, fig. 30b. Within these spectra the expected result of multiple plasmonic modes across the visible spectrum was received. Shown in fig. $30 b$, for $\mathrm{AgNCs}$ the three plasmon modes $\mathrm{D}_{\mathrm{Ag}}, \mathrm{Q}_{\mathrm{Ag}}$, and $\mathrm{M}_{\mathrm{Ag}}$ are present at $418 \mathrm{~nm}, 385 \mathrm{~nm}$ and $343 \mathrm{~nm}$ respectively. Though it was thought that the AgNCs may have lost their cubic features (fig. 29a), depositing these NPs onto PS resulted in the hybridization of these modes and their elucidation was proceeded, fig. 29b. As expected, $\mathrm{D}_{\mathrm{Ag}}$ blueshifts after deposition. AuNPs have a single plasmon mode $\left(\mathrm{D}_{\mathrm{Au}}\right)$ at $532 \mathrm{~nm}(\mathrm{fig} .30 \mathrm{~b})$, blue-shifted $21 \mathrm{~nm}$ from the NPs in solution (fig. 30a). Additional to the desired peaks, there was a broad coupling peak appearing at $\sim 695 \mathrm{~nm}$ (fig. 30b). The specificity of the coupling peak was low; herein different types of clusters $\left(D_{A g}-D_{A g}, D_{A u}-D_{A u}\right.$, and $\left.D_{A u}-D_{A g}\right)$ cannot be distinguished 
spectroscopically, hence they will be summarized to (D-D) for generalization of coupling peaks. Both low and high surface pressures were examined to observe their effects on nanoparticle dispersity and plasmonic response.

At low pressure (0.3-2.0 $\mathrm{mN} / \mathrm{m})$, the coupling peak was lower than the peaks for individual particles (bottom 5 spectra in fig. 29b). Increasing the pressure $(3.0-4.0 \mathrm{mN} / \mathrm{m}$ ) resulted in increases in D-D coupling in this system (top 2 spectra in fig. 30b); D-D coupling intensity increases above the $\mathrm{D}_{\mathrm{Au}}$ intensity. However, as the pressure was increased, there was also changes in the LSPR intensity for individual AgNCs and AuNPs. Considering this, the 1 and $2 \mathrm{mN} / \mathrm{m}$ substrates were selected for further analysis, as their spectra suggested the resonance peak intensity of $\mathrm{D}_{\mathrm{Ag}}$ and $\mathrm{D}_{\mathrm{Au}}$ was higher than that of D-D. Through these experiments, the first hypothesis that by mixing AuNPs and AgNCs, a system containing multiple plasmonic modes spanning greater ranges of the UV-vis spectrum was obtained.

\subsubsection{Characterization of 1:1 Mix of AuNP:AgNC}

The AFM of a $1 \mathrm{mN} / \mathrm{m}$ substrate is presented in fig. 31, showing the different particles contained within the 1:1 mix; different particles have been identified and circled. Different NPs were identified using their $\mathrm{x}$-cross section in the Nova AFM software (top of cross-section is the top of the NP, bottom is the substrate). The nanoparticles have random distribution throughout the substrate (i.e., they have not uniformly ordered themselves). AgNCs were observed throughout, and their shape features are well observed (blue circles in fig. 31). AuNPs have been circled in red in fig. 31 confirming their presence in the sample. 


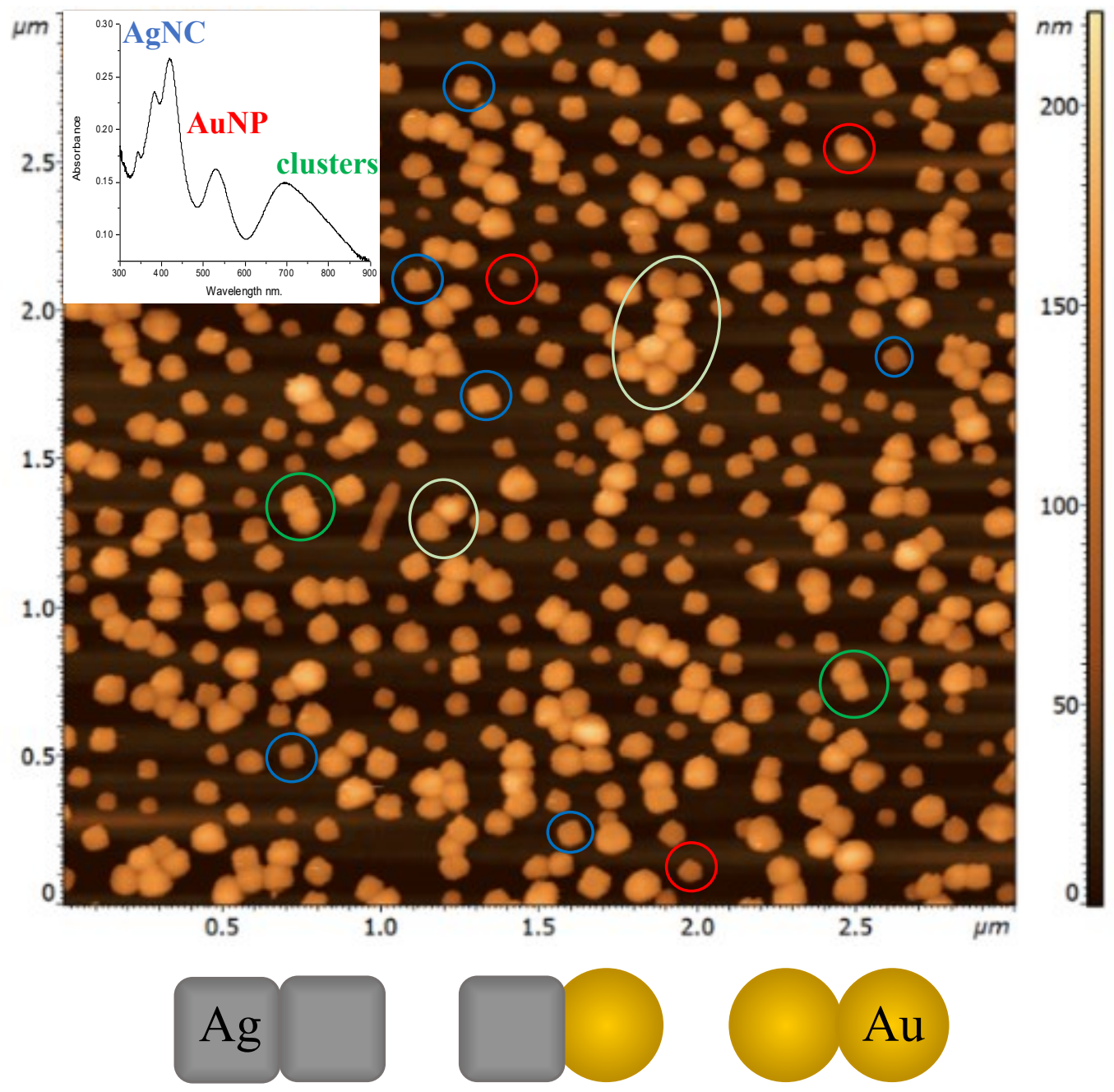

Figure 31: AFM data for a 1:1 Mix of Au:Ag NPs on PS $(1 \mathrm{mN} / \mathrm{m})$, inset spectrum of this slide (top). Bottom shows different types of dimers which occur in the sample.

Total particle density was calculated for individual particles from fig. 31 providing a value of $\sim 11 \mathrm{NPs} / \mu \mathrm{m}^{2}$ for individual particles. Clusters of various size and composition were also observed throughout the sample, including multimers of individual composition (Au or Ag; identified by light green circles) but also multimers of Au and Ag particles, fig. 31. For example, Au:Ag (hetero)dimers can be observed throughout (green circles in fig. 31). Spherical particles have a more rounded distribution, whereas AgNCs have a square-like distribution in their crosssection. In an Au:Ag dimer, the cross-section of an AFM scan contains both parts from AuNPs 
and AgNCs. These cross-sections were used to identify the different types of particles in the sample.

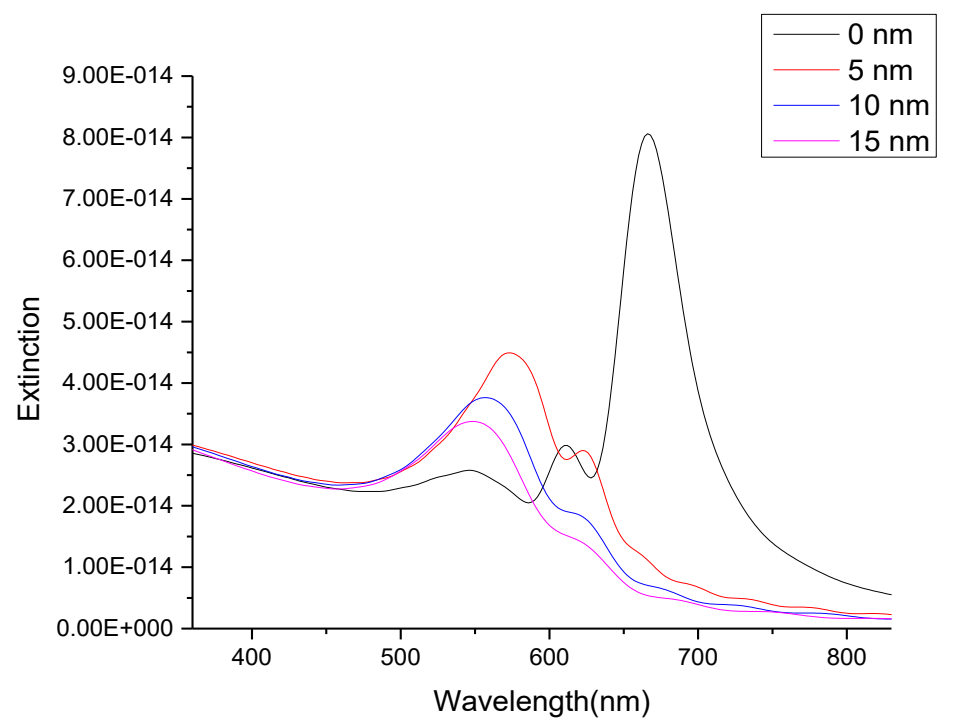

Figure 32: FDTD Model spectra for an Au:Ag dimer at varying distances

The Au:Ag dimer was modelled using FDTD, shown in fig. 32, to observe their dipoledipole coupling, and the effect of separation distance $(0-15 \mathrm{~nm})$ between the nanoparticles on the plasmonic coupling response. Homodimers of both AuNPs and AgNCs have been analyzed and modelled, ${ }^{17,50}$ but there have been no models for heterodimers of AgNCs and AuNPs in the literature. Here, a heterodimer is positioned above a PS film and analyzed for the optical response with increasing NP separation distance. Notably, it requires very powerful computers with vast storage capacity to complete modelling of $\mathrm{Au}$ :Ag heterodimers, as the symmetry filters cannot be applied in the Lumerical FDTD modelling software forcing it to take further discretization.

In fig. 32, sharp resonance corresponding to $\mathrm{D}_{\mathrm{Au}}-\mathrm{D}_{\mathrm{Ag}}$ coupling is observed on the red end of the spectrum $(\sim 690 \mathrm{~nm})$ when the NP distance is $0 \mathrm{~nm}$, as well there is a secondary peak to the left of this resonance. Otherwise, as the interparticle distance is increased the D-D resonance begins to weaken and the plasmon mode blue shifts. This dimer is just one of the scenarios of clusters which would contribute to the coupling resonance. It is important to note that this model 
shows a resonance of greater intensity than that examined for AgNC dimers. ${ }^{139}$ As well, resonances at shorter wavelengths have been observed for AuNP dimers and chains. ${ }^{2,140,141}$

\subsubsection{Conventional Heating of 1:1 Mix}

This research was designed to analyse the optical properties of substrate supported mixtures of $\mathrm{Au}$ and $\mathrm{Ag}$ nanoparticles. A key result of this work was to observe changes in the plasmonic response of these nanoparticle mixtures upon changing the local environment.

a)
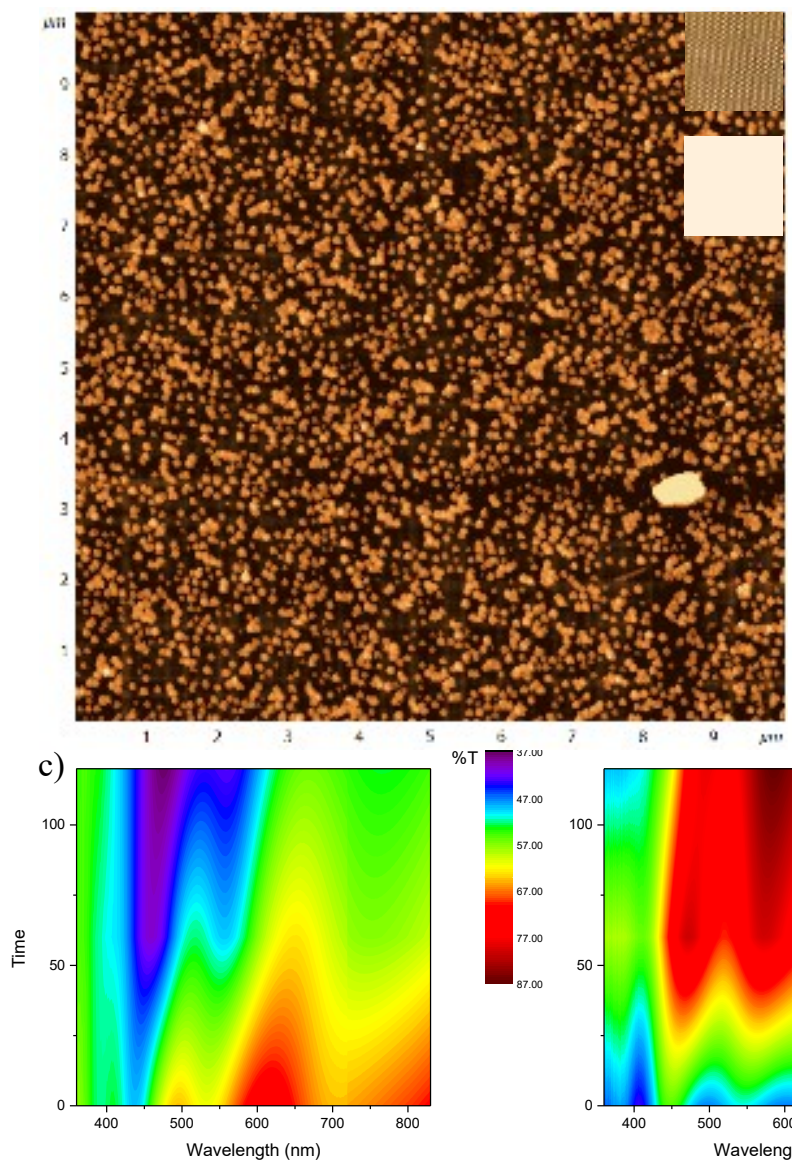

b)
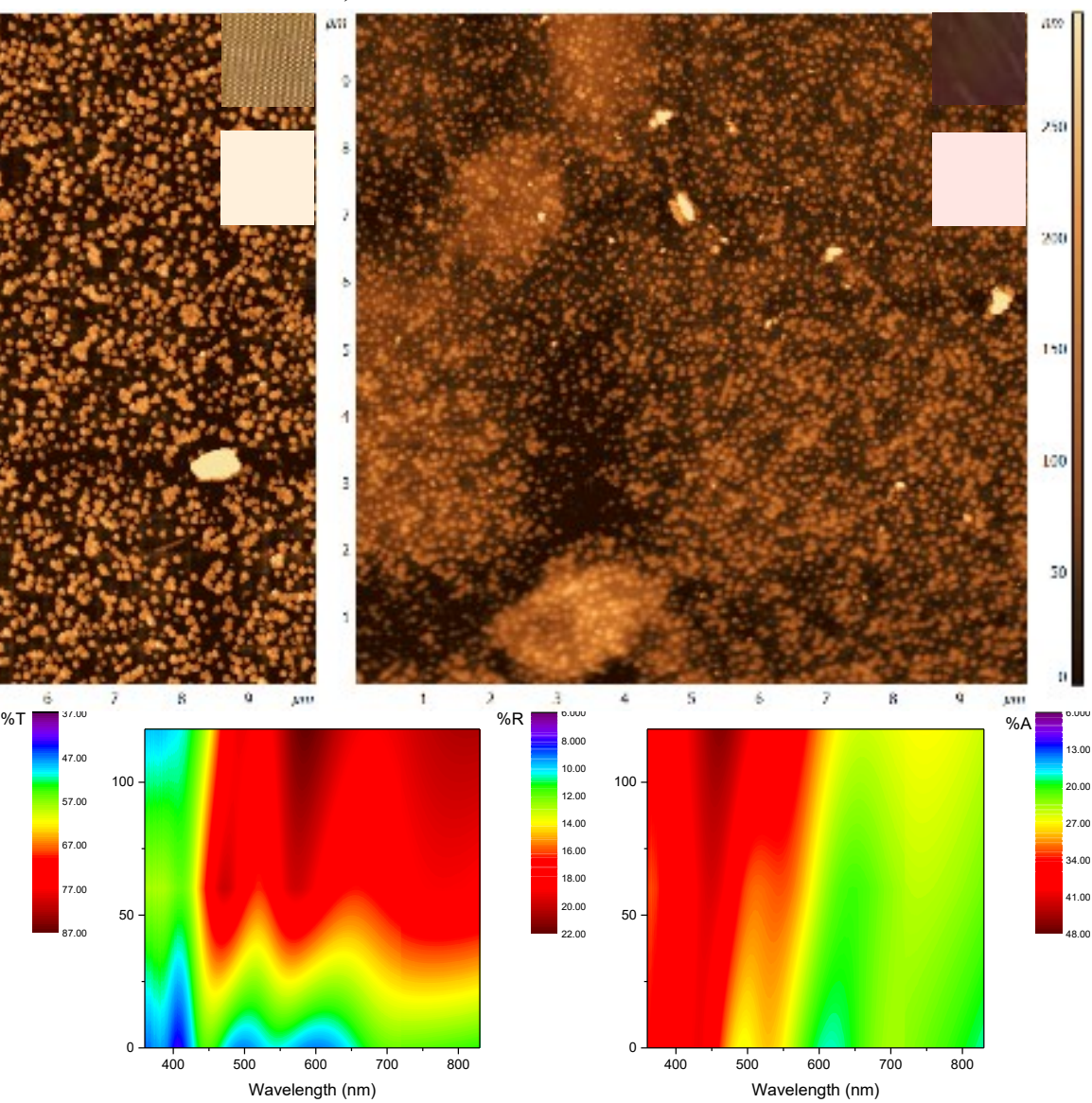

Figure 33:AFM images of 1:1 Mix on PS $(2 \mathrm{mN} / \mathrm{m})$ a) before and $\mathrm{b}$ ) after heating, inset photographed colour (above) CIE 1931 colour representation (below) of the substrates and c) Transmission, Reflectance and Absorptance spectra of heating the slide for $120 \mathrm{~s}$ at $\sim 135^{\circ} \mathrm{C}$.

Heating has been the primary source thus far for observing effects of changing the local environment of the NP on the optical properties exhibited; the like was conducted for the mixture on PS substrates. Figure 33a, and $b$ show the AFM images for the 1:1 mix before and after heating 
respectively. Upon heating a $1 \mathrm{mN} / \mathrm{m} \mathrm{1:1} \mathrm{mix} \mathrm{substrate,} \mathrm{distinct} \mathrm{changes} \mathrm{in} \mathrm{the} \mathrm{colour} \mathrm{of} \mathrm{the}$ material were noticed. Moving from being yellow-beigey in colour to a more pink/red coloured material (inset colours, fig. 33a, and b); the colour change occurred in the experiment and are shown in the CIE representation (below) and in images of the actual substrates (above).

An interesting observation of note is that AgNC substrates have a yellow colour and AuNP substrates are pink/red coloured. Fig. 33c shows the transmission, reflectance, and absorptance spectra as the substrates are heated over time. Transmission (fig. 33c, left), of light through the substrate is observed to decrease as the nanoparticles embedded. In the case of reflectance (fig. $33 c$, middle), and absorptance (fig. 33c, right), it was noticed that both the $\mathrm{D}_{\mathrm{Ag}}$ and $\mathrm{D}_{\mathrm{Au}}$ underwent significant increases in intensity, alongside LSPR red shifts, with increasing embedment. These results suggests that when the nanoparticles are above PS, AgNCs absorptance dominates and after embedment AuNP reflectance dominates. The effect of colour here is dictated by the dipolar reflectance; increasing reflectance at the dipolar mode is the perceived colour due to nanoparticles embedding into the polymer.

\subsection{Spectrally Selective Thermoplasmonic Embedment}

Further to the conventional heating of these slides (i.e., on a hot plate), these materials can also be used to test their potential for spectrally selective thermal embedment. The thermoplasmonic effect can be used to manipulate the nanomaterials in a more controlled fashion than conventional heating (which results in the embedment of all particles simultaneously). Using the thermoplasmonic effect, specific wavelengths of light can be selected to excite the NP LSPR resulting in heat generation. Herein, the selected substrates are of interest as they contain multiple plasmon modes available to be manipulated. With mixtures of Au and Ag NPs, it was expected that selecting a blue wavelength for the laser $(458 \mathrm{~nm})$ would result in the selective 
thermoplasmonic embedment of AgNCs, whereas selecting a wavelength in the yellow region (568 nm) would result in AuNP embedment.

\subsubsection{Selective embedment in NPs in 1:1 mix at $458 \mathrm{~nm}$}

A $1 \mathrm{mN} / \mathrm{m}$ substrate of the 1:1 mixture, was irradiated with a laser focused on a wavelength of $458 \mathrm{~nm}$. The laser power was measured immediately before the focusing lens using a power meter. The power measured for these experiments was $22 \mathrm{~mW}$, as it was a power low enough to cause heating of cubes without complete thermal ablation of the polystyrene film. It was determined that a power of $22 \mathrm{~mW}$ would not cause thermal ablation by testing various powers until the system was optimized to only embed the nanoparticles. To maximize thermoplasmonic heating, the selection of wavelengths corresponding to the dipolar resonant frequency is important. ${ }^{63,64,72,142}$ In this system, LSPR for $\mathrm{D}_{\mathrm{Ag}}$ was $418 \mathrm{~nm}$ suggesting that the cubes should be excited at this frequency. Previous work showing thermoplasmonic embedment of AgNCs on PS was conducted at a wavelength of $458 \mathrm{~nm}$ and showed efficient embedment of the NCs at a power of $17 \mathrm{~mW} .{ }^{139}$ The lowest accessible wavelength in the given set-up is $458 \mathrm{~nm}$, it was utilized here with a slightly higher power $(22 \mathrm{~mW})$.

Fig. 35 shows a summary of the thermoplasmonic embedment at $458 \mathrm{~nm}$ from $0-55 \mathrm{~s}$. Initially, the particles are above the PS layer (fig. 35a) measuring an average AgNC height of 65.4 $\pm 3.6 \mathrm{~nm}$. After $15 \mathrm{~s}$ of continuous wave laser exposure there is a distinct change in the substrate (fig. 35b); the nanoparticles had begun to embed near the center of the laser, and the AFM image suggests that more than just NCs were embedding. More importantly, the height profile changes, identifying the occurrence of embedment as the height moved to $36 \pm 3 \mathrm{~nm}$. The entire scope of the laser exposure at $458 \mathrm{~nm}$ can be observed in Section 6, fig. 53. 
a)

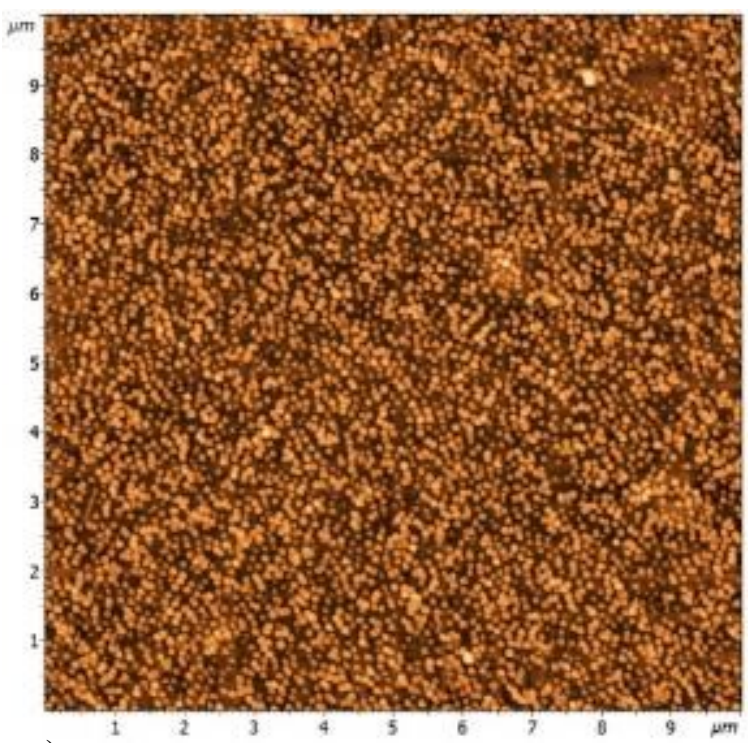

c)

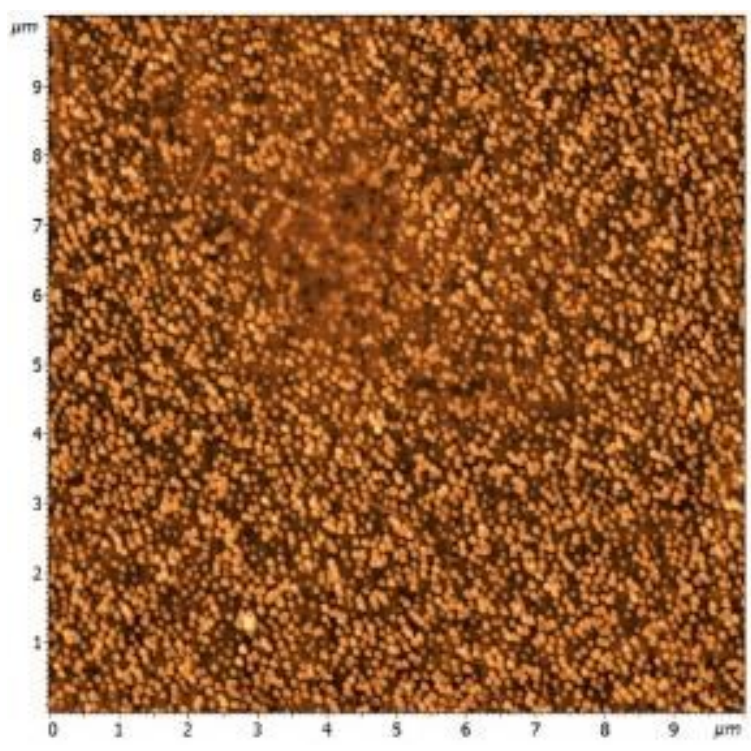

b)

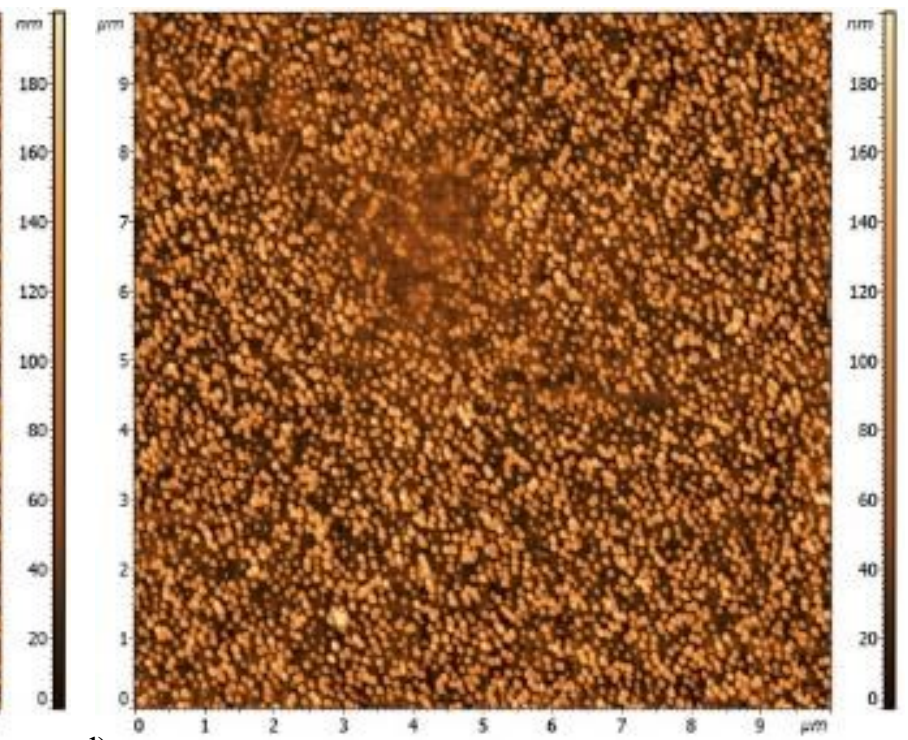

d)

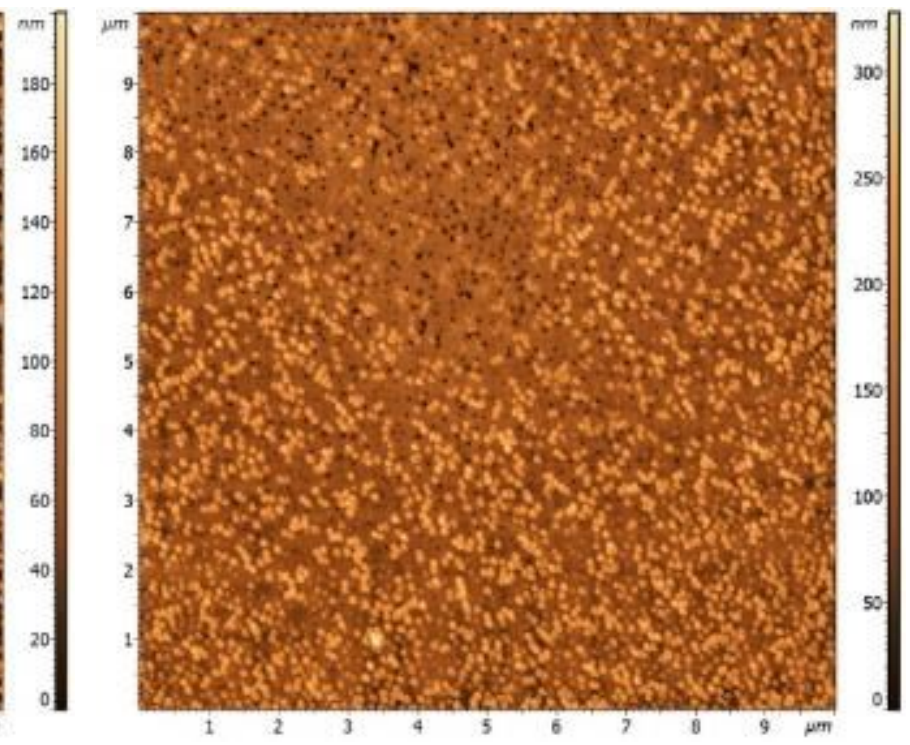

Figure 34: AFM images of 1:1 mix on PS after $458 \mathrm{~nm}$ laser exposure (22 mW) for a) $0 \mathrm{~s}, \mathrm{~b}) 15 \mathrm{~s}, \mathrm{c}) 30 \mathrm{~s}$, and d) $55 \mathrm{~s}$

After only $15 \mathrm{~s}$ there are particles which are partially embedded into the polymer film. Though it is of importance to note that some of the polymer has been heated enough by the AgNC thermal conversion to result in the embedment of other nanoparticles in the vicinity (not just individual AgNCs), observable in fig. 34b. At the $30 \mathrm{~s}$ interval, fig. 35c, holes are observed in the substrate. These holes are AgNCs which have been excited sufficiently high enough that their 
thermal radiation transfers to the polymer, and continuously. In $30 \mathrm{~s}$ there are many observable holes in the film, all having different heights (e.g., holes of depth $53 \mathrm{~nm}, 30 \mathrm{~nm}$, and $17 \mathrm{~nm}$ were found). The holes can be observed in the cross-section as a deep peak below the surface of the polymer.

In figure 35d, it was observed that further exposure with the laser for $55 \mathrm{~s}$ produced a vast number of AgNC holes in the film. Interestingly, many of these holes have gone past the height of the cube, signifying that the cubes will continue to embed after increased laser exposure, a result consistently observed in the literature. ${ }^{70}$ These experiments confirm not only the embedment of AgNCs into the polymer, but also the location of individual AgNCs on the substrate (most perceivable in fig. 35d) This selective thermoplasmonic process resulted in the rapid embedment of AgNCs into the polystyrene film and aided in confirmation that there were individual nanocubes present. The condition which suggested that NPs need to be excited at their resonance wavelength for maximum temperature conversion efficiency still stands. Though, it should be stated that it is not necessary that the AgNCs be excited at their exact resonate frequency to yield the thermoplasmonic effect (e.g., AgNCs having a on substrate LSPR of $418 \mathrm{~nm}$ can be excited to emit heat with neighbouring wavelengths).

\subsubsection{Selective embedment in NPs in 1:1 mix at $568 \mathrm{~nm}$}

The irradiance of the same $1 \mathrm{mN} / \mathrm{m} \mathrm{1:1} \mathrm{mix} \mathrm{substrate}$ with a laser of wavelength $568 \mathrm{~nm}$ was also carried out. By irradiating the slide with $568 \mathrm{~nm}$ it was intended to excite AuNPs sufficiently well enough to embed them into the PS film as with silver NCs. Here, $\mathrm{D}_{\text {Au }}$ was located spectrally at a wavelength of $532 \mathrm{~nm}$. Laser lines are discrete and there are only so many in vicinity of the resonance peaks, but plasmonic resonances are broad enough to be excited at neighbouring wavelengths. Hence a wavelength of $568 \mathrm{~nm}$ was selected for the thermoplasmonic embedment of 
AuNPs, though it is of noteworthy mention that in previous experiments this wavelength was utilized for thermoplasmonic excitation of AgNC clusters using a power of $65 \mathrm{~mW} .{ }^{139}$

a)

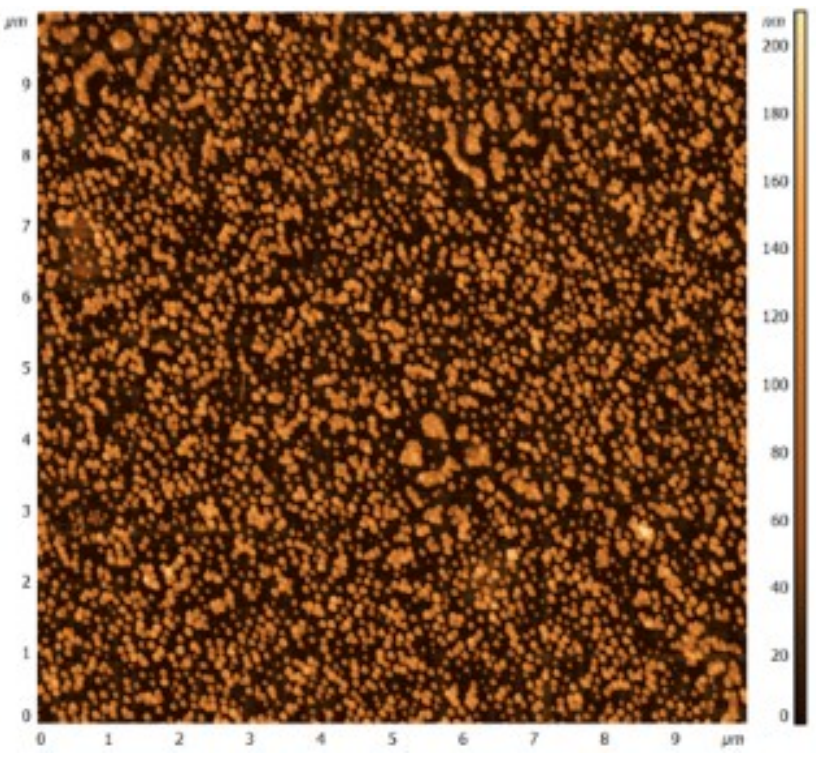

b)

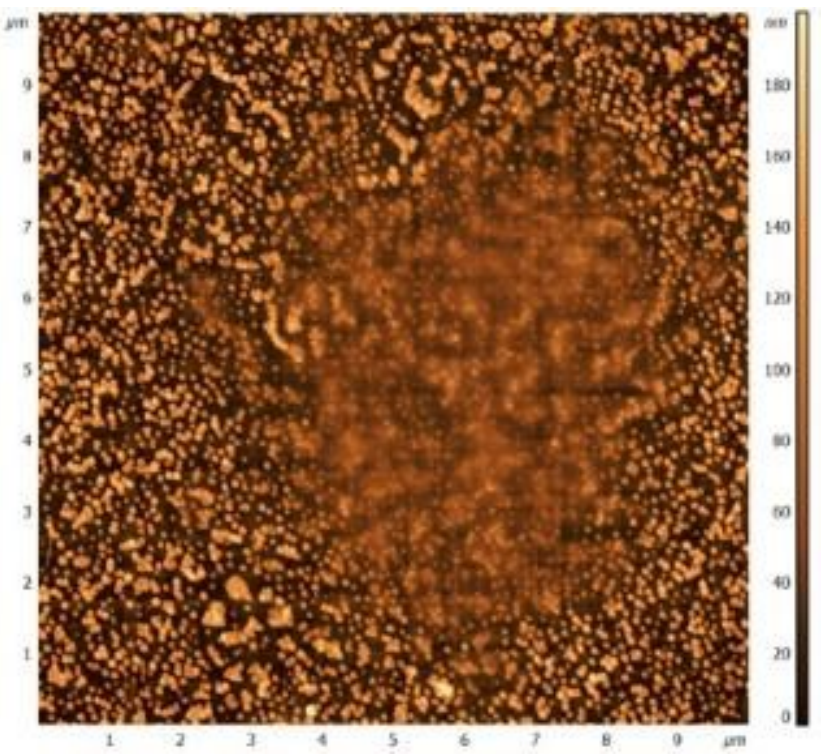

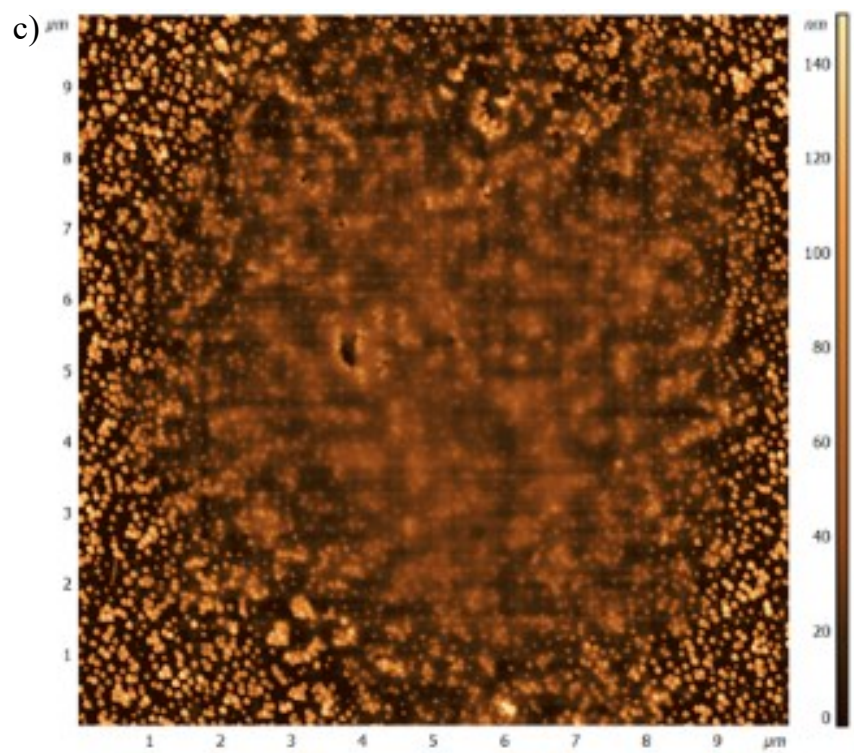

Figure 35: AFM images and respective cross-sections of 1:1 mix on PS after $568 \mathrm{~nm}$ laser exposure (50 $\mathrm{mW}$ ) for a) $0 \mathrm{~s}$, b) $50 \mathrm{~s}$, and c) $6 \mathrm{~min}$.

Herein, the slide was exposed to the laser with $50 \mathrm{~mW}$ of power for times ranging from 0 6 min. The summary is presented in fig. 36, but the entire set of experiments can be observed in section 6, fig 54. The results obtained in the $568 \mathrm{~nm}$ laser exposure differ vastly from the $458 \mathrm{~nm}$ experiments. In this case the embedment of individual particles, and subsequent formation of holes 
does not occur. Rather the system results in the total heating of the area that experienced laser irradiance.

Initially, the slide had a distinct profile with an average height of $67.3 \mathrm{~nm}$. Individual particles as well as clusters can be observed based on the AFM data given in fig. 36a. After laser exposure at $568 \mathrm{~nm}(50 \mathrm{~mW})$ for $50 \mathrm{~s}$ there is obvious embedment of nanoparticles observed. The average height measured was $40.2 \mathrm{~nm}$. This suggested that the nanoparticles had embedded into the polymer film. Through review of $f i g .36 b$, it can be stated that more than just individual AuNPs had embedded. The embedment of AuNPs, clusters and any other particles contained in the exposure area had occurred. Prolonged exposure with the laser to 6 minutes only resulted in the same situation, but more nanoparticles near the outer ends of the laser beam had also embedded. No holes were observed here suggesting that the excitation wavelength was not specific enough to result in only individual AuNPs being heated. Rather this wavelength caused excitation of AuNPs and clusters.

From this, it is understood and confirmed that a $568 \mathrm{~nm}$ wavelength will result in thermoplasmonic heating of i) the AuNPs, ii) the clusters, or iii) both. From fig. $36 c$ it was observed that individual AuNPs embedded into the polymer, but not to the extent that AgNCs embedded (forming holes). However, the embedment of clusters is also perceived. It is known that larger particles (including clusters) can more rapidly exhibit photothermal heating. Selecting this spectral position for the laser, in this system, likely resulted in cumulative local heating of the polymer due to high thermalization from clusters, thus the nanoparticles embed similarly to as observed in conventional heating. The final height of the nanoparticles on the substrate after 6 min (fig. 36c) was $33.6 \mathrm{~nm}$. So, it is possible to manipulate the system at this wavelength, however, the AuNPs require greater selectivity of wavelength if it is desired to observe their individual embedment. 
Indeed, the distribution of heat throughout the polymer was observed within the $458 \mathrm{~nm}$ experiments, though not to this extent. Hence, more fine tuning of the wavelength for selective thermoplasmonic embedment of AuNPs is needed. In future experiments using a laser exposure at $518 \mathrm{~nm}$ may be desired to embed only individual AuNPs.

\subsubsection{Selective embedment with switching laser from $568 \mathrm{~nm}$ to $458 \mathrm{~nm}$}

The mixtures of $\mathrm{Au}$ and $\mathrm{Ag}$ nanoparticles have shown interesting optical responses to light.

By changing their local environments (e.g., embedding NPs into the polymer), a method for controlling the plasmonic response across a broad range of the electromagnetic spectrum has been produced. Manipulation of nanoparticles using thermoplasmonic embedment offers vast opportunity for nanopatterning affording precise control and selection of the state of the nanoparticles. The excitation of the 1:1 mix substrate with a $458 \mathrm{~nm}$ laser resulted in the embedment of the individual AgNCs resulting in deep holes being formed in the PS film upon thermal dissipation. The same sample irradiated with a $568 \mathrm{~nm}$ laser also resulted in nanoparticle embedment but was not as selective as the former (individual nanoparticles and clusters embedded).

In consideration of the provided results, and given the synthesized materials (1:1 Au:Ag mix), the possibility to embed different particle types into the polymer was possible by selecting the specific excitation wavelength (dipolar LSPR) of the particles desired to be embedded. Therefore, it is possible to manipulate, within a single sample with multiple plasmon modes, different modes at their respective resonances to result in their selective embedment. Plainly, it is possible to first embed one particle type (e.g., AuNPs) using one wavelength first, then switch the laser's wavelength to the other excitation position to embed the desired particles (e.g., AgNCs). An exemplary result of this is shown in fig. 37 where the first wavelength selected was $568 \mathrm{~nm}$ (intending to embed AuNPs), then the wavelength was switched to $458 \mathrm{~nm}$ to embed AgNCs. 
a)

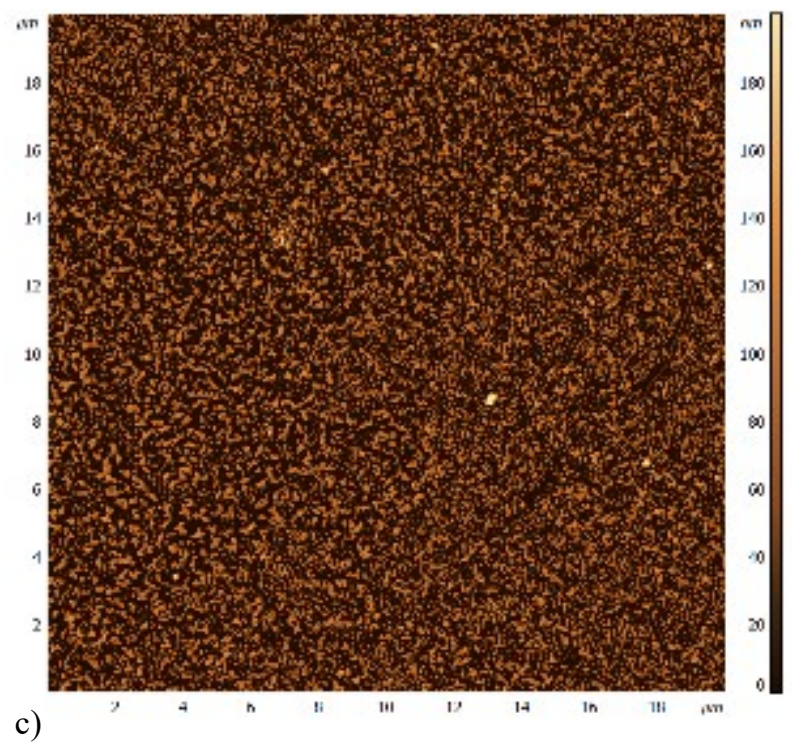

c)

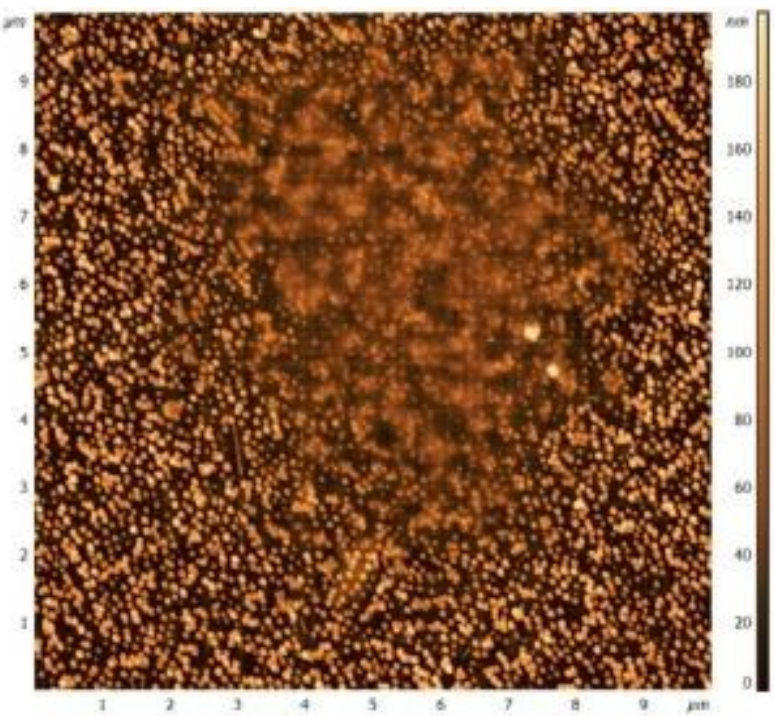

b)

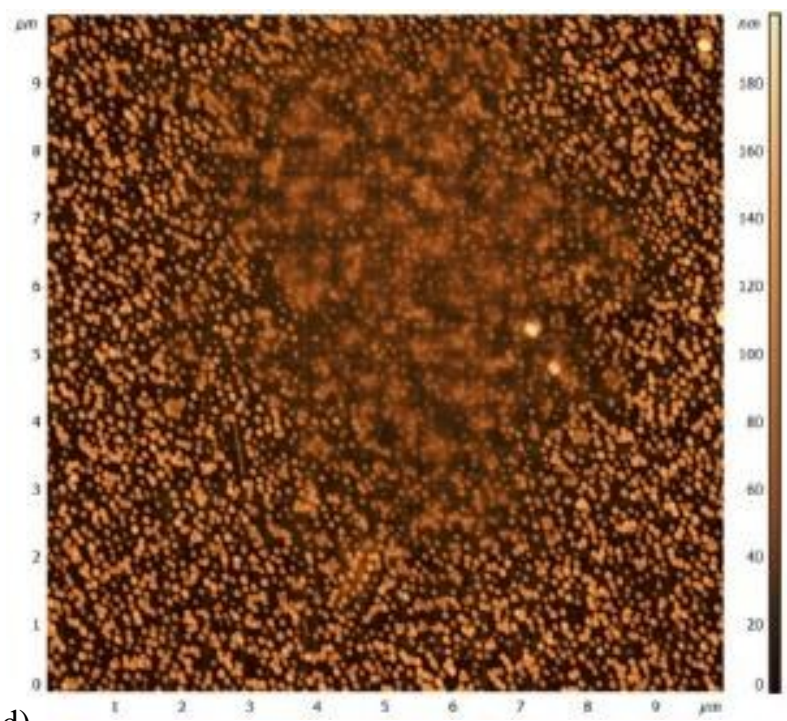

d)

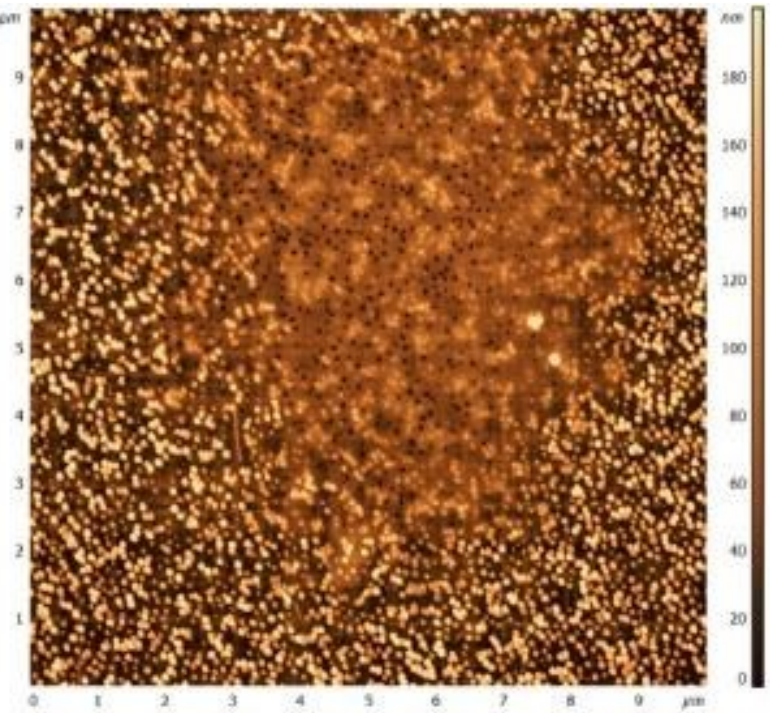

Figure 36: AFM images of 1:1 mix on PS after $568 \mathrm{~nm}$ laser exposure (50 mW) for a) $0 \mathrm{~s}, \mathrm{~b}) 15 \mathrm{~s}$, and subsequent $458 \mathrm{~nm}$ laser exposure (22 $\mathrm{mW}$ ) for c) $10 \mathrm{~s}$ and, d) $1 \mathrm{~min}$.

When first under laser exposure at $568 \mathrm{~nm}(50 \mathrm{~mW})$, an effect like what was observed in the $568 \mathrm{~nm}$ experiments occurs. Here, the slide was only exposed to this laser for $15 \mathrm{~s}(\mathrm{fig} .37 \mathrm{~b})$, which was sufficient for embedment of the particles in the area. Like the $568 \mathrm{~nm}$ experiments, the AFM image suggests that there is enough local heating to result in the embedment of surrounding particles. Importantly, this does not have an impact on the ability to embed individual AgNCs with laser exposure at $458 \mathrm{~nm}(22 \mathrm{~mW})$. Since the embedment process had already begun, because of 
local heating when the substrate was excited at $568 \mathrm{~nm}$, the AgNC thermoplasmonic embedment $(458 \mathrm{~nm})$ only needed $10 \mathrm{~s}$ to start forming holes in the polymer (fig. $37 \mathrm{c})$. After $1 \mathrm{~min}$ of laser exposure in the same area, fig. 37d, a large quantity of AgNCs had embedded, creating a bounty of holes in the PS film. The entire set of laser exposures can be seen in figure 55.

These results confirmed the ability to embed the different types of particles within one system using the thermoplasmonic effect to do so. Hence, there exists new opportunity for nanopatterning (specifically thermoplasmonic patterning) using substrate supported mixtures of $\mathrm{Au}$ and Ag NPs. Therefore, the second prediction that these plasmonic modes be tunable across the UV-vis-NIR spectrum as the NP local environment is manipulated was confirmed.

\subsection{Gap Plasmons: New Nanoarchitectures for Colour Generation}

An interesting phenomenon is the multiple cavity-like resonant mode, known as the gap mode. The gap mode is the plasmonic response resulting from the coupling of two plasmonic materials which have been separated by a gap distance. ${ }^{143,144}$ Herein, a thin $(\sim 50 \mathrm{~nm})$ reflective metal mirror is coated with an ultra-thin $(\sim 30 \mathrm{~nm}$ or less $)$ polystyrene film, then topped with a monolayer of nanoparticles. The metal mirrors used are composed of either Ag or Au; selected due to their large quality factor. The gap mode existence has been observed, and proved in many instances. ${ }^{143-146}$ Prezgot, Ianoul, et al., ${ }^{147}$ have shown the ability to use hybrid plasmon resonances to probe a strong optical response as a consequence of the gap mode formation. Specifically, AgNCs over either an Au or Ag thin film were used and separated by polystyrene (16-20 nm thick). The FDTD models for these experiments have been reproduced in fig. 38 where $60 \mathrm{~nm}$ edge length AgNCs with a $2 \mathrm{~nm}$ PVP overcoating, have been separated by ultra-thin PS films over a metal (either $\mathrm{Au}$ or $\mathrm{Ag}$ ).

In the case of AgNCs above an Au substrate, fig 38 (left), a gap mode is observed starting near $550 \mathrm{~nm}$ with a $16 \mathrm{~nm}$ gap distance (between the NP and MS). As the gap distance is decreased 
there is an increase in the coupling response between the two objects. The gap mode for AgNCs on an Au film spans the region from $\sim 550 \mathrm{~nm}$ to $850 \mathrm{~nm}$ in this system (fig. 38 (left)), presenting an increasingly sharp resonance above $550 \mathrm{~nm}$. For AgNCs atop an Ag film, fig. 38 (right), sharper resonances are observed giving a gap which spans $\sim 480$ to above $800 \mathrm{~nm}$. The AgNC on $\mathrm{Ag}$ (fig. 38 (right)) system produces the gap mode sooner than on an Au film (fig. 38 (left)), and over a larger range.
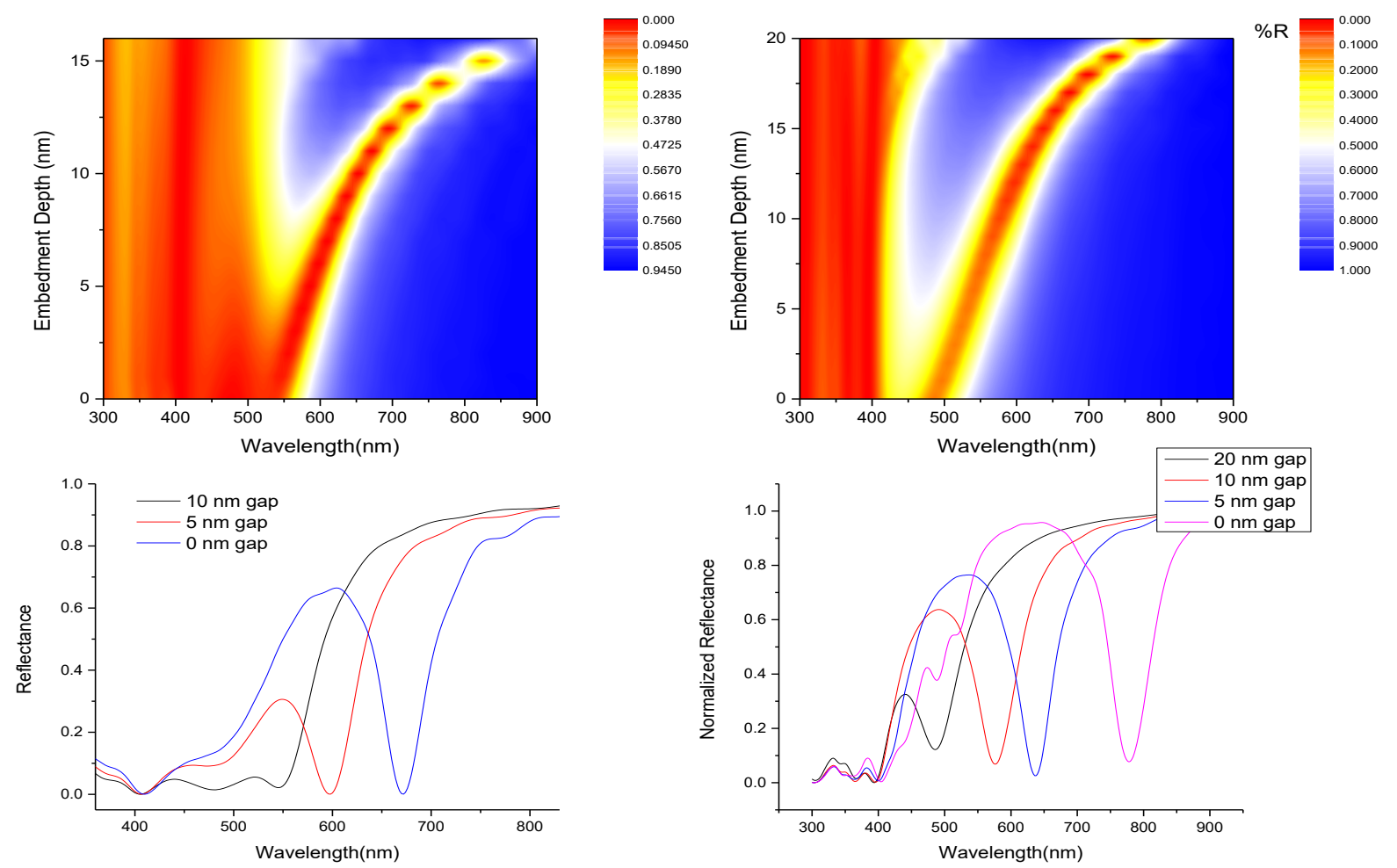

Figure 37: FDTD Model for Reflectance spectra of $60 \mathrm{~nm}$ AgNCs on $50 \mathrm{~nm} \mathrm{Au}$ (left), and $50 \mathrm{~nm} \mathrm{Ag} \mathrm{(right)}$ with respect to a change in the gap distance $(\mathrm{nm})$ by embedment into a $16 \mathrm{~nm}$, and $20 \mathrm{~nm}$ PS film respectively.

These models informed the ability to utilize PS as the dielectric spacer layer to form sharp gap resonances, with Ag having the most noticeable changes in the gap mode as the nanoparticles are brought closer to the metal surface. The gap distances are controlled by embedment of nanoparticles into the polymer film, thus bringing them closer to the metal film. It has already been proved that the nanoparticle embedment can be proceeded using conventional heating or they can 
undergo precise control of their embedment through photothermal heating (i.e., the thermoplasmonic effect). So, the gap distance can be manipulated by thermal embedment. Herein, only conventional heating was utilized to change the gap distance, though photothermal heating would also work and offers control of the state of the nanoparticles with greater precision.

An important result of the gap mode is the intense, and strong colours produced due to the coupling between the NP LSPR and the SPP of the MS. This is an extension of plasmonic colour generation extending it further than say just the observed colour when increasing nanoparticle size, changing nanoparticle shape or even interparticle coupling. Plasmonic colour is advantageous as it has longevity and demonstrates properties like iridescence or dichroism. In contrast to the more common dye-based pigments, plasmonic colouring yields high resolution colours and is more environmentally stable. By changing the gap distance, a variety of colours is produced, allowing not only colour generation, but also its fine control.

\subsubsection{AuNPs on Au thin film}

When AuNPs are modelled on top of an Au film separated by a $20 \mathrm{~nm}$ PS layer, the FDTD model provides a description of the expected result, shown in fig. 39. It is first observed that when the gap distance is large $(20 \mathrm{~nm})$, coupling between the objects is weak, producing a broad response in the reflectance (fig. 39 (right)). As the gap distance is made shorter, the gap mode becomes more prevalent. Unlike in the AgNC systems, where a broad range of sharp and very different gap modes exist, with AuNPs on Au there is only evidence of a weak gap mode spanning a short range (fig. 39 (left)). Although, this short range and "weak" gap mode are still very much gap resonances. Moreover, these gap modes do produce a variety of colours (fig. 39 (bottom)) as the particles moves closer to the metal surface. The CIE 1931 colour space was used to produce the representative colours based on the reflectance data; this system explores wavelengths between 360-830 nm. 

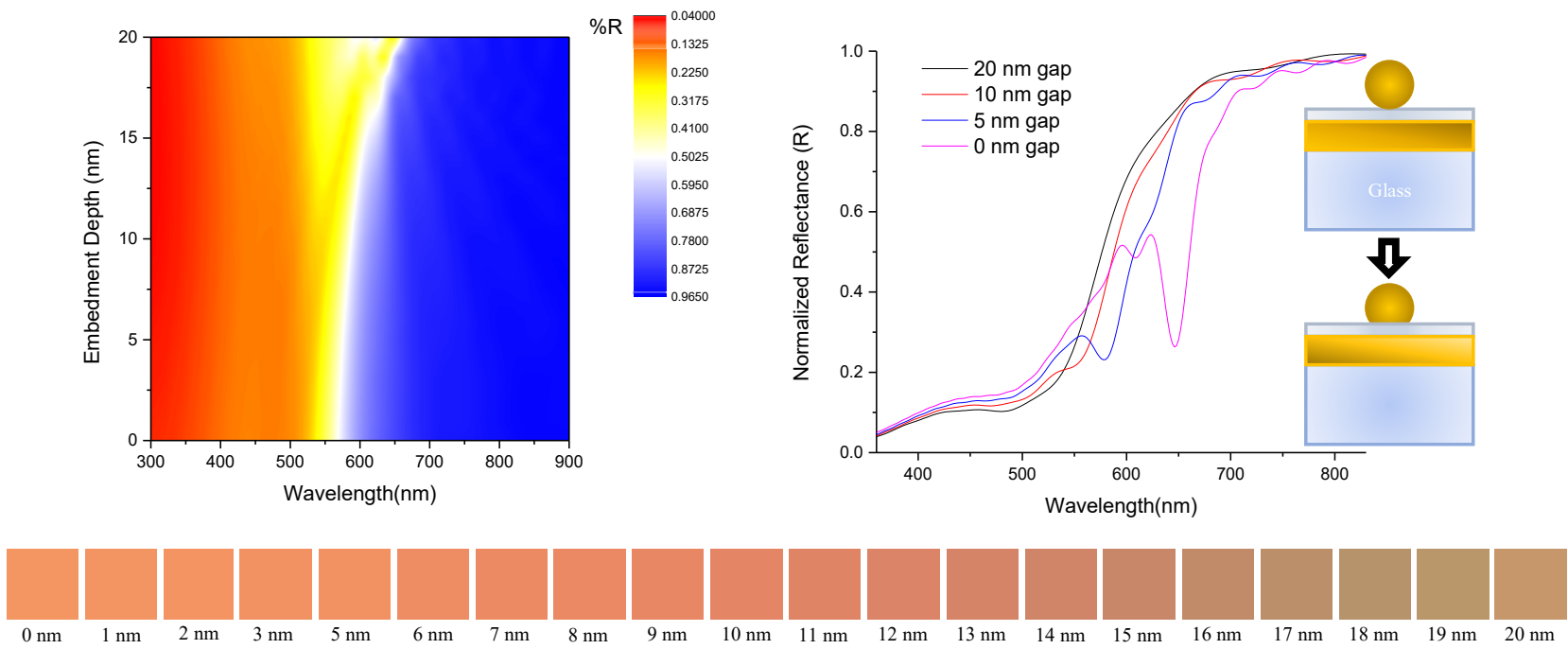

Figure 38: Reflectance spectra of the FDTD model of AuNPs on Au film with respect to a change in the gap distance $(\mathrm{nm})$. The CIE representation of the predicted colours is below and is a function of embedment depth $(0-20 \mathrm{~nm})$

The experimental results for AuNPs on an Au film are shown in figure 40. In experiment, the gap mode is observed, but provides weaker (broader, less intense) comparative response in their reflectance; the model predicts the resonances will be sharper, and that the gap mode should begin to appear before $600 \mathrm{~nm}$ (fig. 39 (right)). The weak binding in the experimental system is attributed to rather high size distribution of AuNPs, as well as some of the response being lost to the D-D coupling mode (fig. 40 (right)). The reflectance spectra, fig. 40, suggest that there is broad contribution of the D-D mode to the gap resonance. In fact, fig. 40 (left) shows that the coupling peak seems to have formed its own (weak) gap mode with the metal surface ranging from 800 to $1150 \mathrm{~nm}$. As a result, the range of colours obtained experimentally was different than what was observed in the model, since the coupling was within the range for inputted colour data.

Though the entire predicted range of colours (fig. 39, bottom) was not observed, similarities in the represented colours (fig. 40, top) for experimental and model were be noticed. Specifically, closer to the end of both ranges of colour the similarities were observed. As the slide was heated the coupling peak moved further into the IR region, and this is the probable reason colours like the 
expected result become more visible since the coupling effect moved out of the visible region (fig. 40 (right)).
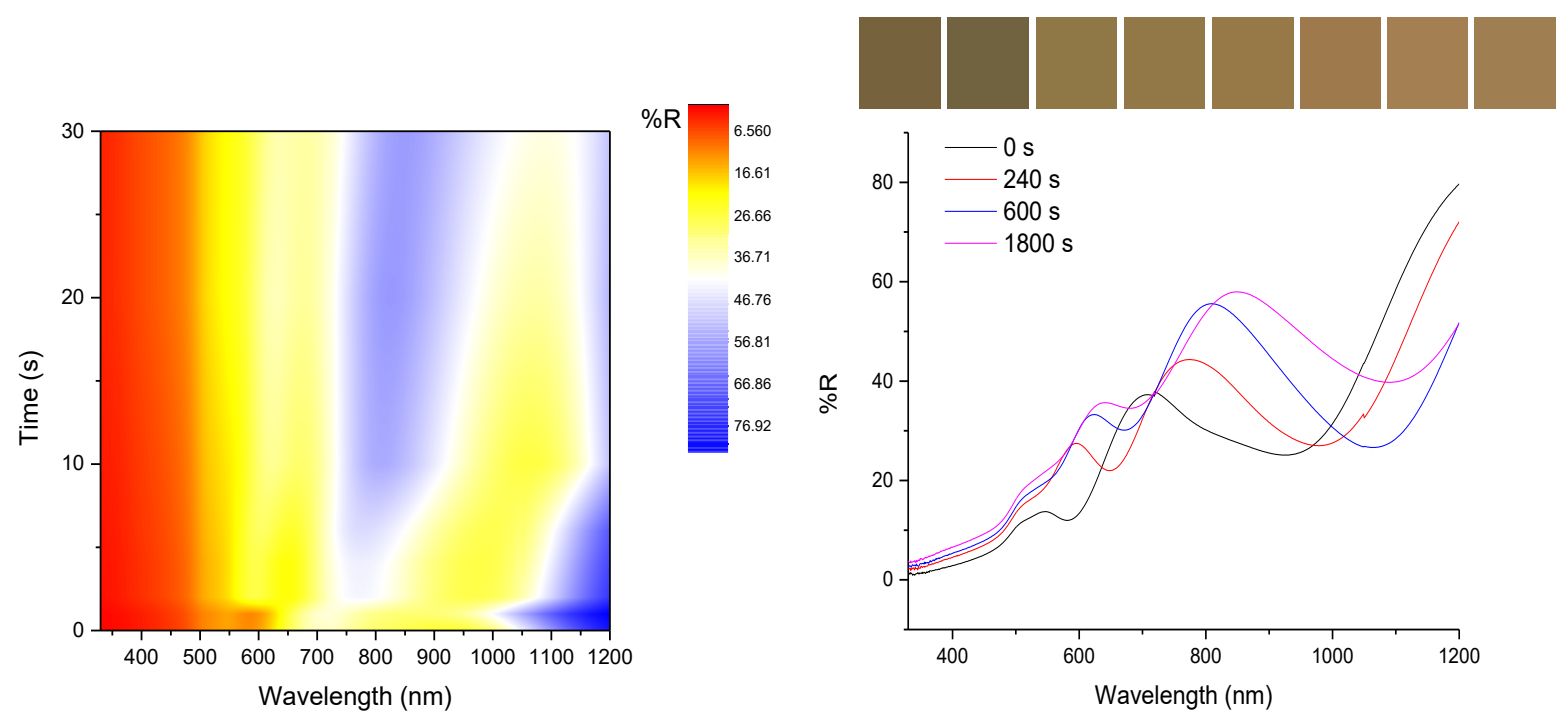

Figure 39: Reflectance spectra of the Exp results of AuNPs on Au film with respect to a change in the gap distance (nm).

Considering the model, it becomes necessary to review the thickness dependence of the spacer layer on the resultant gap mode between AuNPs and the metal film. The dielectric spacer layer thickness has been observed to have a major impact on the gap mode. ${ }^{59}$ Information gained from modelling the thickness dependence will aid in the fine tuning of the system, and optimization for the design of mixtures over metal films.

\subsubsection{AuNPs on Ag thin film}

Figure 41 shows the FDTD model for the reflectance of AuNPs above a $50 \mathrm{~nm} \mathrm{Ag} \mathrm{film}$ and their CIE predicted colours. The reflectance spectra monitoring the embedment of NPs over time, fig. 41 left, presents the appearance of a gap resonance with increasing intensity, spanning 2500-600 nm. Fig. 41 (right), confirmed that the gap mode should red shift with decreasing gap distance. The colours yielded from the model of AuNPs on Ag (fig. 41, bottom) have indiscernible 
changes at far distances from the MS ( 1-7 nm embedment depth), but as the embedment depth increases to $\sim 8-20 \mathrm{~nm}$ the colour changes become more distinguishable (from pink to grey).
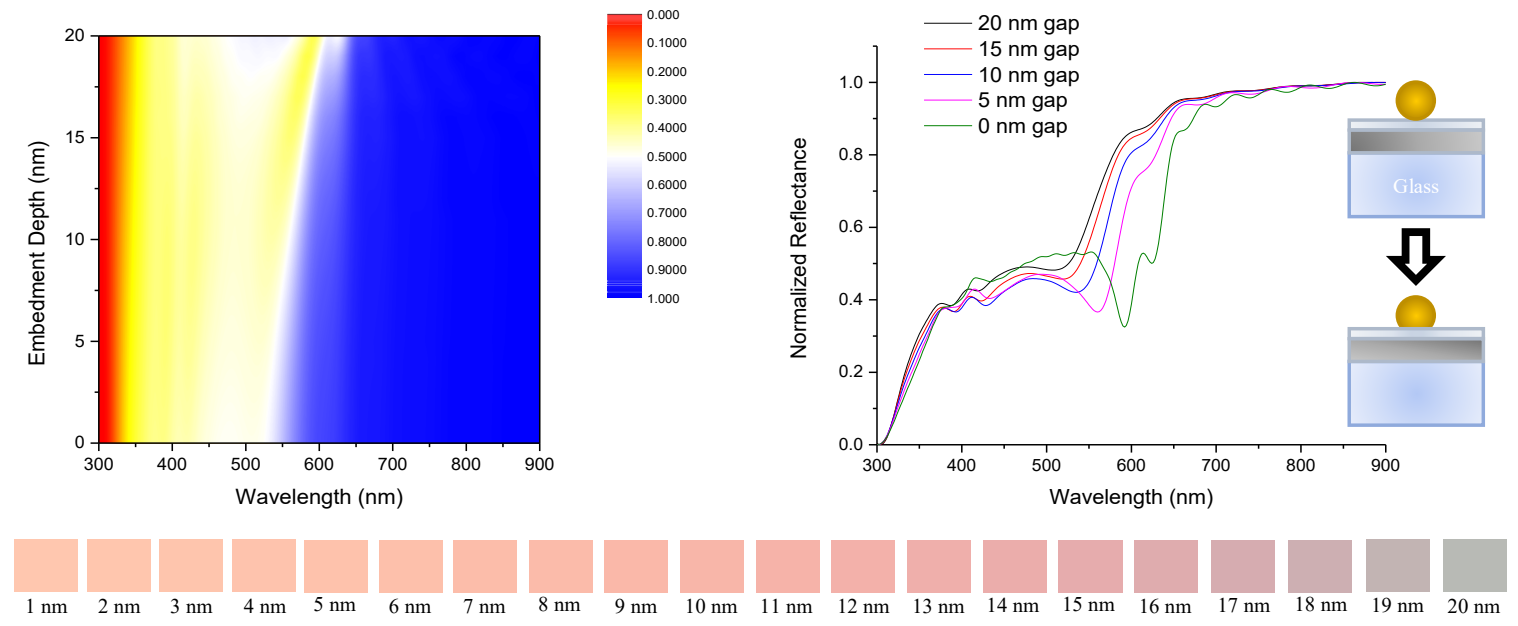

Figure 40: Reflectance spectra of the FDTD model of AuNPs on an Ag film with respect to a change in the gap distance $(\mathrm{nm})$. The CIE representation of the predicted colours is below and is a function of embedment depth $(0-20 \mathrm{~nm})$ shown below spectra.

The experimental reflectance measurements for AuNPs on an Ag film are presented in figure 42, with the CIE represented colours shown above the spectra. As the substrate containing AuNPs on an Ag film is heated there are minute changes in the gap mode. The obtained reflectance spectra, fig. 42 right, suggest that there were not significant changes in reflectance as the substrate was heated; the gap mode began at $\sim 580 \mathrm{~nm}$ and remained below $600 \mathrm{~nm}$. The gap resonance was also noticed to be broad and weak in this system. Moreover, the coupling peak still exists in this system and had an impact on the perceived colour. The colour range is not large experimentally, likely a result of coupling.

In comparison to the model, fig. 41, the gap mode experienced does not undergo major changes. It was expected that there be a gap resonance of increasing intensity as the NP embedment depth increased, but in experiment the resonance was observed to both broadened and decrease in intensity (fig. 42 (right)). Of noteworthy mention, the model depicts a uniform system of 
nanoparticles, and so it does perfectly represent the experimental results, which contained coupled nanoparticles. Hence, the colours yielded in experiment were different than the theoretical predictions based on the FDTD model.
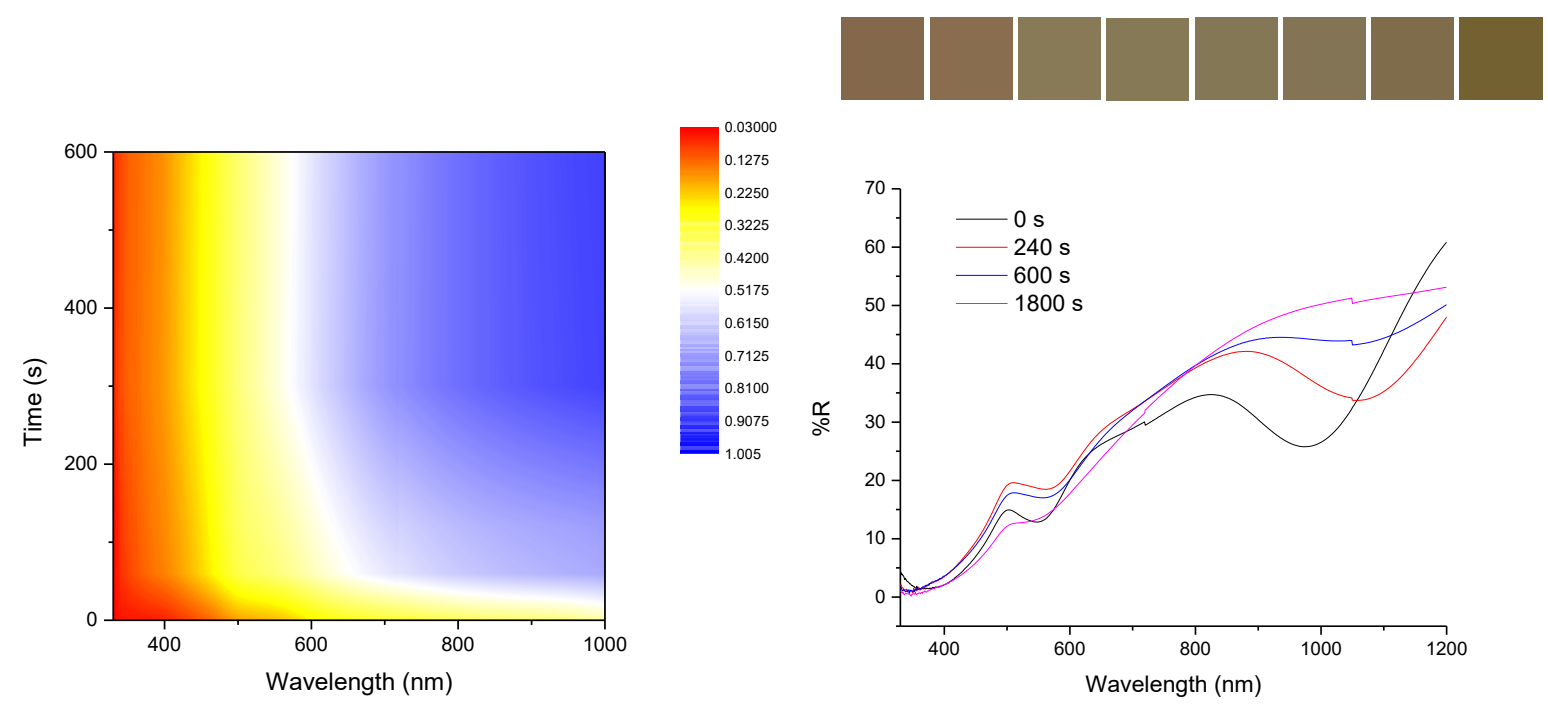

Figure 41: Reflectance spectra of the Exp results of AuNPs on Ag film with respect to a change in the gap distance (nm).

\subsubsection{Mix on Au thin film}

Now that the gap plasmonic response has been understood for both AgNCs, and AuNPs on metal surfaces, it becomes plausible to use these effects for plasmonic colour generation using mixtures of Ag and Au NPs. It was expected that, using mixtures of nanoparticles, two distinct gap modes would appear. With two gap modes, there is possibility to control (even further) the optical response of the nanomaterials. More specifically, if photothermal embedment were utilized, it would be possible to just embed AgNCs or just embed AuNPs (or even clusters if desired) to manipulate the gap distance and fine time the optical properties of the material.

The FDTD model of a mix of AuNPs:AgNCs above an Au film and its change in reflectance as the nanoparticles approach the metal is given in fig. 43. Initially, at a gap distance of $15 \mathrm{~nm}$, there were no distinct, discernible peaks in the reflectance spectrum, but over time the gap mode starts to appear. Based off the model reflectance spectra for individual NPs, the modes 
present in this mix were elucidated. Most of the reflectance comes from AgNCs as it can form coupling modes to the metal surface sooner (resulting from the hybrid plasmon modes). This is observed spectrally in fig. 43. As well, a broad set of colours was plausible using the mixture, especially when compared only to AuNPs on a MS (fig. 39, and 41).
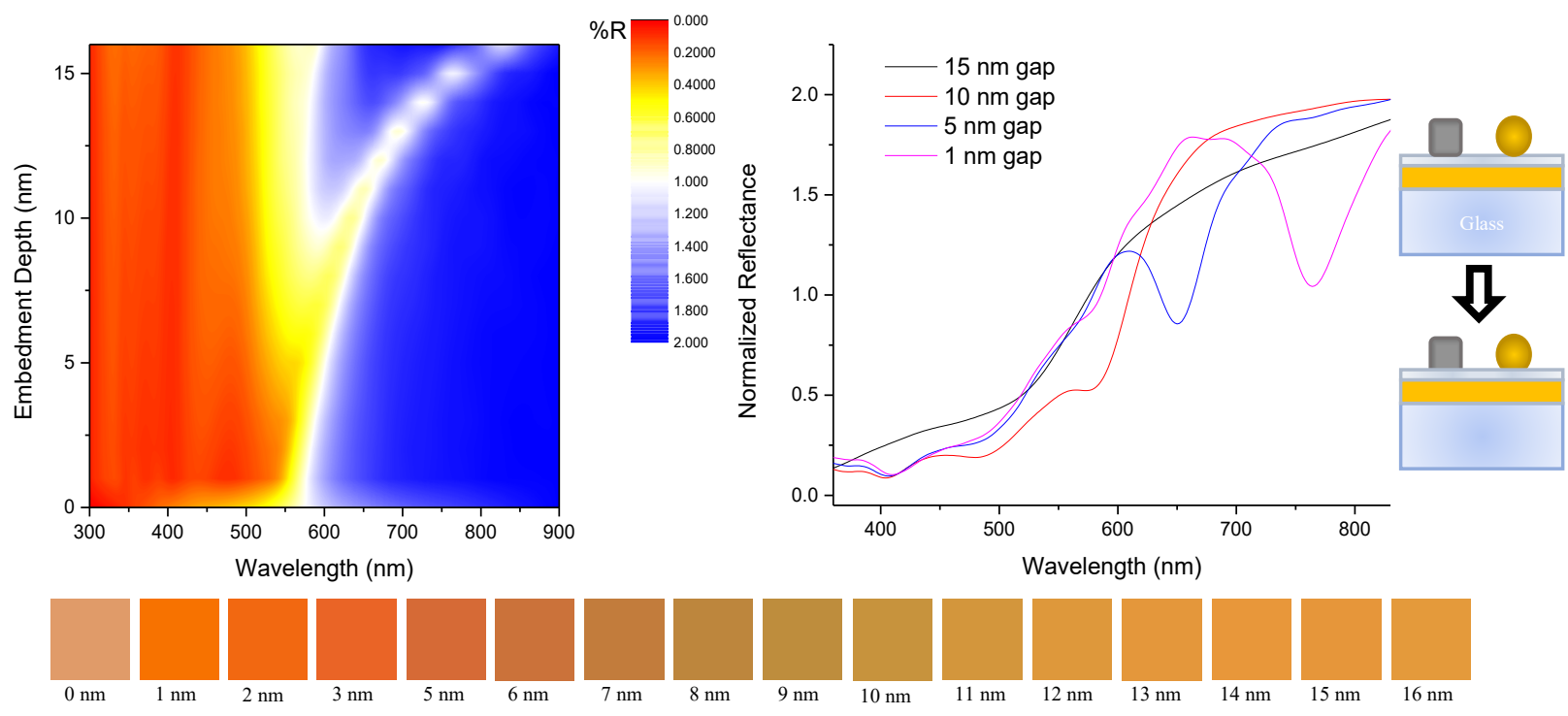

Figure 42: Reflectance spectra of the FDTD model of a 1:1 Mix on an Au film with respect to a change in the gap distance $(\mathrm{nm})$.

The AuNP gap mode is not easily perceivable but exists and contributes a fair amount to the colour. There were only small changes in the reflectance of AuNPs initially, but after a while (at a gap distance of $1 \mathrm{~nm}$ ) shows the second gap mode near the initial reflectance of AgNCs, fig. 43 (right), though the peak is not well-resolved. At far distances, (>10 nm gap), it was likely the gap modes of AuNPs and AgNCs had overlapped, hence, only one mode was observed. Since Au is not hybridized, the gap mode is not as strong as for AgNCs but is still prevalent and manipulatable. The model proves the ability to use mixtures of nanoparticles for multiple gap mode generation to be used to greater tunability in plasmonic colouring.

In experiment, fig. 44, a 1:3 mix of AuNPs:AgNCs was used in attempt to curb the coupling which was observed to come (predominantly) from Au containing clusters. A small gap mode, 
likely for AgNCs, was observed in fig. 44 (right) beginning before $600 \mathrm{~nm}$ (as seen for the model of AgNC gap mode in a mix at a gap of $10 \mathrm{~nm} ; 5 \mathrm{~nm}$ embedment depth, fig. 38 (left)). Showing how the reflectance spectra change over time, fig. 44 (left), identifies that a gap mode was existent in the sample, though mode was not as dignified as in previous cases. The remaining colours in the range only come from a system which represents a gap resonance for AgNCs at resonances above $600 \mathrm{~nm}(\sim 630 \mathrm{~nm})$. The CIE representation of the colours are not at all the same as the predicted colours, but there is some inkling that system is attempting to fit within the regime (between 5-10 nm embedment depth). Although, the initial state of the actual slides (fig. 44 (top left)) appears to have colouring like the FDTD predicted colour (fig. 43 (bottom)) of the $0 \mathrm{~nm}$ embedment depth. The final state of the substrate has colouring nearing the middle $(\sim 7-10 \mathrm{~nm}$ embedment) of the range of predicted colours. This suggests that with careful preparation of welldispersed nanoparticles on the substrate would lead to a sample more closely related to the model.
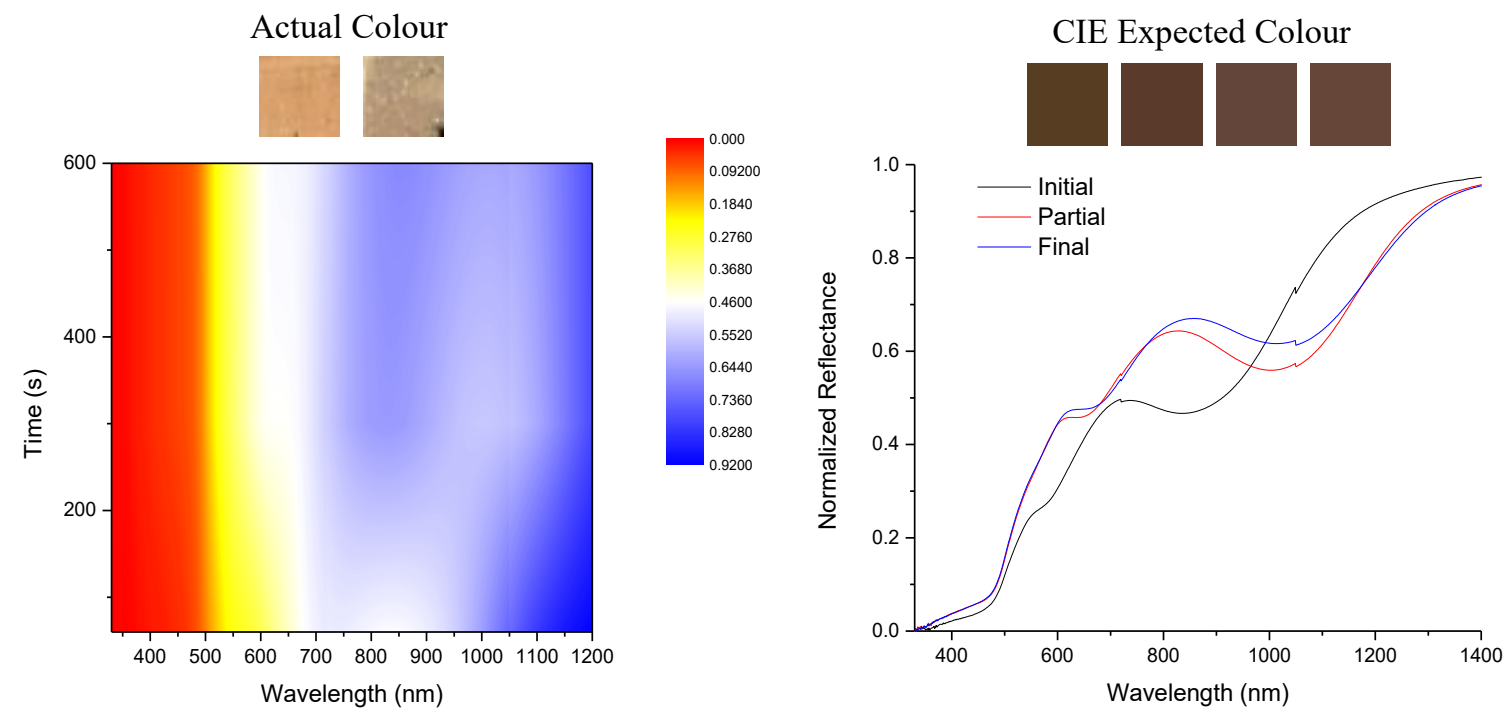

Figure 43: Reflectance spectra of the Exp results of the 1:3 Mix on an Au film with respect to a change in the gap distance $(\mathrm{nm})$. The actual colours of the substrates are shown above the spectra (top left). The CIE representation of the colours is presented above the spectra (top right) 
D-D coupling was concluded to mask the colour that should be yielded in a well-dispersed monolayer. Fig. 45 shows an AFM image of the 1:3 mixture on an Au film. In this image individual nanoparticles, as well as clusters can be observed. There is a large quantity of clusters on this substrate and their contributions to the plasmonic response are very apparent. Nonetheless, the gap mode was formed between the mixture and metal film resulting in some colour generation (different from the in-solution and on PS colours). The benefit of the Au films is that they were very smooth, hence the SPP was consistent and propagated well along the MS resulting in fair gap resonances. Moreover, the model is representative of an even distribution of nanoparticles in a 1:1 ratio. Since the model is for a 1:1 mixture and the actual experimental method used a 1:3 Mix, it was also expected that there be some variation from the model. Still, less clusters and narrow size distribution would lead to the formation of a more consistent system that matches more closely that of the model.

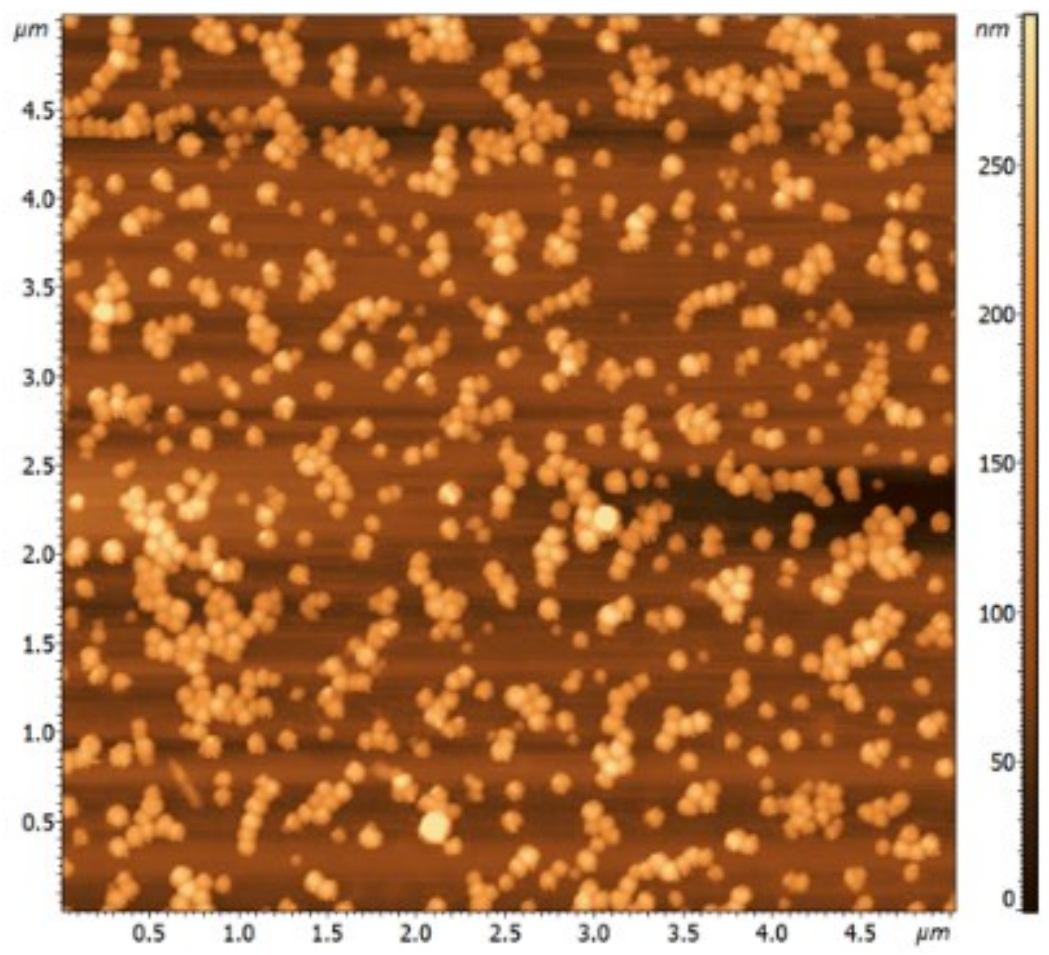

Figure 44: AFM image of a 1:3 mixture of Au:Ag on an Au film coated with 25 nm PS. 


\subsubsection{Mix on Ag thin film}

The FDTD model for the reflectance measurements of the NP mix is observed in fig. 46.

At $20 \mathrm{~nm}$ from the metal film, there was instantaneously a gap mode present (fig. 46 (right)). As the particles are brought closer to the MS, the gap mode red shifts, and the resonance intensity appeared to decrease. Given a $5 \mathrm{~nm}$ gap distance, the appearance of a second mode, adjacent to the red shifting gap mode was noted. That second mode represents the gap resonance between AuNPs and the Ag film. The modes become more distinguished at a $0 \mathrm{~nm}$ gap. In observation of the reflectance change over time, it was noticed that initially there was one gap mode, but as the nanoparticles are brought closer to the surface there should be two gap modes present (one for AgNCs and one for AuNPs). Remarkably, colours predicted from the model, fig. 46 (bottom), span a range much larger than observed in the prior experiments; a wide variety of colours is accessible with a mix above an Ag film.

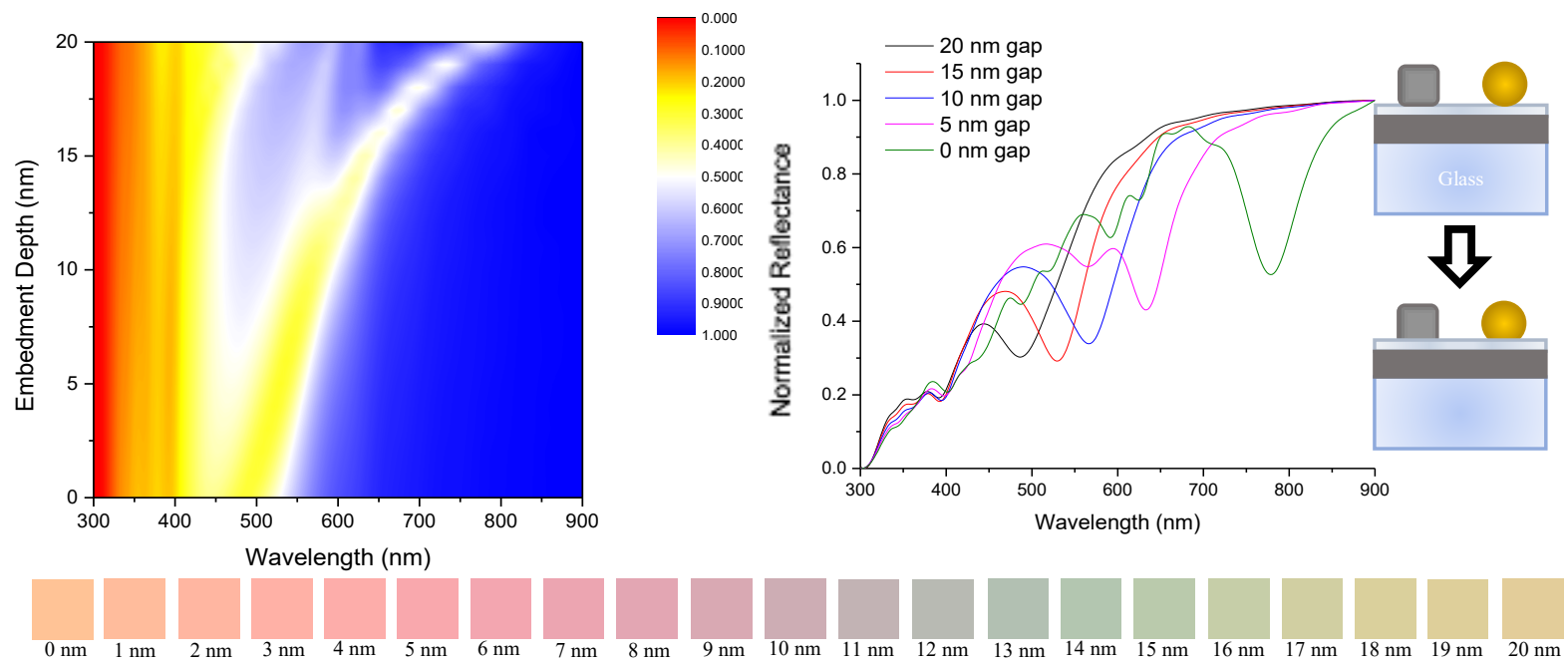

Figure 45: Reflectance spectra of the FDTD model of a 1:1 Mix on an Ag film with respect to a change in the gap distance $(\mathrm{nm})$.

The experimental reflectance spectra for the deposition of a 1:3 mix is given in Fig. 47. There were not significant changes in the reflectance as the substrate is heated; these results were the most uncharacteristic of marvelous gap modes. Though there were gap modes observed here, 
they were extremely weak and seemed to continue to weaken as the slide was heated. When mapped to the CIE 1931 colourspace, the representative colours show no remarkable changes after the initial heating (fig 47 (top)). Reviewing the actual colour of the substrates (fig. 47 (right)) there are more notable similarities in the colours of the initial and final state to the FDTD predicted colours (fig. 46 (bottom)). This suggests that some of the individual nanoparticles were able to couple and form a gap mode.

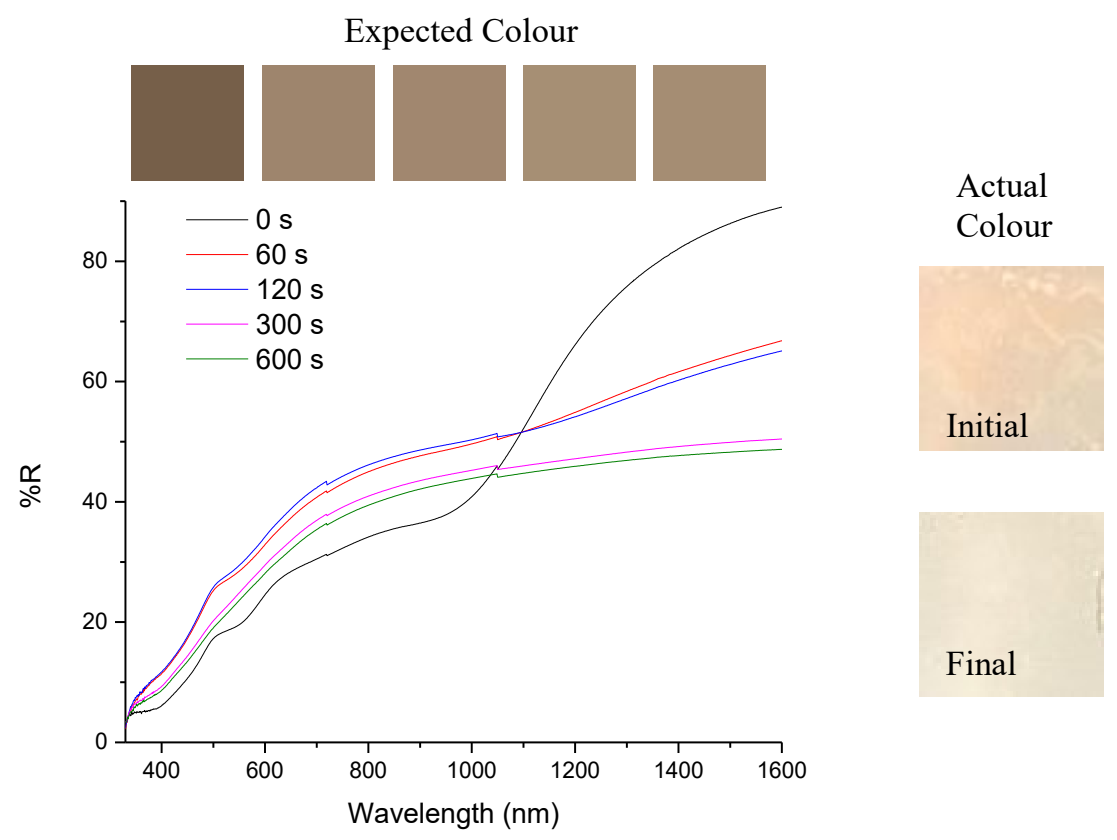

Figure 46: Reflectance spectra of the Exp results of the 1:3 Mix on an Ag film with respect to a change in the gap distance (nm). The CIE representation of the colours is presented above the spectra. The actual colours of the substrates are shown adjacent the spectra.

As can be observed in the AFM image of the 1:3 mixture on a silver film (fig. 48), there are inconsistencies in the film. Instead of a continuous film, there were many silver islands which compose the film. An inconsistent metal film means that the plasmons cannot propagate throughout the film. Therefore, the strength of the gap mode was decreased by the roughness of the film. The model (fig. 65) is representative of a smooth Ag film, hence the variance in result was expected. As well, from fig. 48, many coupled NPs were observed which would also diminish 
the formation of the gap mode and subsequent control via nanoparticle embedment. Nonetheless, the FDTD model (fig. 45) suggests that mixtures of Ag and Au NPs above an Ag film would yield a large variety of colours and deserved further exploration.

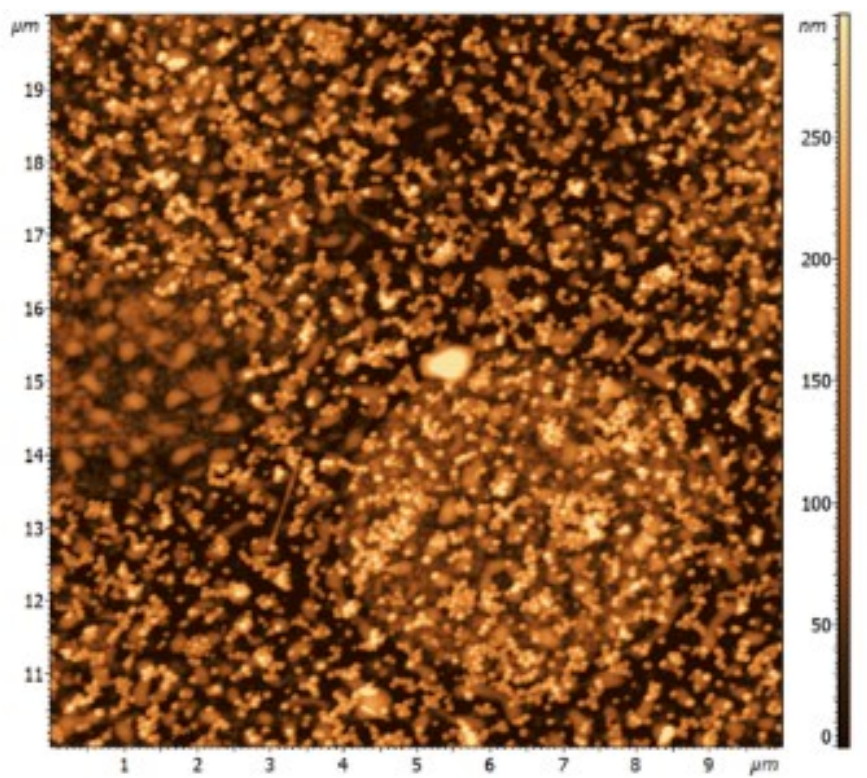

Figure 47: AFM image of a 1:3 mixture of Au:Ag on an Ag film coated with 25 nm PS.

Through experimental results and theoretical modelling, the third hypothesis that mixtures of $\mathrm{Au}$ and Ag nanoparticles can be used to probe a plasmonic gap mode between the NPs and a MS. Thus, plasmonic colouring can be achieved with greater tunability. Expanding the ways in which the light is manipulated, localized, and observed by means of plasmonics.

\section{Conclusions:}

These experiments were conducted to analyze the optical properties of mixtures of $\mathrm{Au}$ and Ag nanoparticles and manipulate those optical properties to the desired need. Mixtures of Ag and $\mathrm{Au}$ NPs were formed in 1:1 extinction ratio. Particularly, AgNCs and AuNPs were mixed after determination of their extinction coefficients, $7.69 \times 10^{-11} \mathrm{~cm}^{2} /$ particle and $4.17 \times 10^{-11} \mathrm{~cm}^{2} /$ particle respectively. In solution their extinction profile showed dipolar LSPR peaks at $440 \mathrm{~nm}$ for AgNCs and $553 \mathrm{~nm}$ for AuNPs, moreover, there was some inkling of multipolar and quadrupolar 
resonance in their regions. Though it appeared that there may have been a loss in the cubic features, due to the weakened multipolar resonance, deposition of the mixture on a PS film showed that the cubes were still intact. On the substrate, these nanoparticles retained their strong dipolar resonances at $418 \mathrm{~nm}$ and $532 \mathrm{~nm}$ for AgNCs and AuNPs respectively. Interestingly, the multipolar and quadrupolar resonances appear, confirming that there were nanocubes in the system, and that plasmon hybridization in the nanocubes still occurred in the mixtures. A dipoledipole coupling peak was also observed in these 1:1 mixtures. Heating these substrates above the $\mathrm{T}_{\mathrm{g}}$ of polystyrene resulted in their embedment into the polymer film. When the entire substrate is heated via conventional heating, there are corresponding changes to their optical responses; specifically, the substrates change colour (yellow to pink) as the nanoparticles are embedded into the polymer - a shift in response to changes in their local environment. Alike was conducted photothermally, using spectrally selective thermoplasmonic heating to release enough heat to the polymer, locally, causing the nanoparticles to embed. These results showed that it is possible to select specific wavelength to embed different types (composition, or clusters) of NPs. When a wavelength of $458 \mathrm{~nm}(22 \mathrm{~mW})$ was selected for selective embedment, individual AgNCs were noticed to embed, creating holes in the film (as expected). At $568 \mathrm{~nm}(50 \mathrm{~mW})$, it was predicted that individual AuNPs would embed - forming holes, though what was received was something like what is achieved in the conventional heating. This was due to the coupling of the nanoparticles which can also have resonances at or near $568 \mathrm{~nm}$. Heating these particles as well, resulted in enough collective local heating to embed anything contained in the laser area. If intended to embed individual AuNPs with laser irradiation, there will be a need to select a wavelength closer to its resonance as AuNPs seem to need more specific selection of a wavelength than AgNCs. Nonetheless, it is possible to embed i) individual particles, and ii) particles of different 
composition. An interesting application of these mixtures employed herein, is their deposition over thin metal films $(\sim 50 \mathrm{~nm}$ Au or Ag) separated by a thin polystyrene film $(<30 \mathrm{~nm})$. These new nanoarchitectures can generate plasmonic gap modes, resulting in generation of a variety of colours. As the nanoparticles are brought closer to the metal surface, the gap mode was expected to red shift. This was observed for the Mix on Au system, but the Mix on Ag results are inconclusive as the Ag films were not smooth enough to exert a collective response. FDTD modeling of these systems suggest that there is possibility to obtain gap modes in the mixtures, producing varying colours which were predicted using CIE 1931 to represent the colour. When these colours are compared to the experimental colours there are variances from the predicted colours. Although, there is still a gap mode present in all systems, and it is noticed to experience changes as the nanoparticles embed into the polymer. In the future, there needs to be more work to reduce aggregates, which formed once the nanoparticles were deposited on substrate, from the sample. This would provide more well-dispersed materials which could be used to embed not only individual AgNCs, but also individual AuNPs. As well, provide a more consistent monolayer for coupling to a metal film, producing coloured material much closer to what was predicted in the model. 


\section{References:}

(1) Zhang, Q.; Moran, C. H.; Xia, X.; Rycenga, M.; Li, N.; Xia, Y. Synthesis of Ag Nanobars in the Presence of Single-Crystal Seeds and a Bromide Compound, and Their SurfaceEnhanced Raman Scattering (SERS) Properties. Langmuir 2012, 28 (24), 9047-9054. https://doi.org/10.1021/la300253a.

(2) Huang, X.; El-Sayed, M. A. Gold Nanoparticles: Optical Properties and Implementations in Cancer Diagnosis and Photothermal Therapy. J. Adv. Res. 2010, 1 (1), 13-28. https://doi.org/10.1016/j.jare.2010.02.002.

(3) Ahamad, N.; Bottomley, A.; Ianoul, A. Optimizing Refractive Index Sensitivity of Supported Silver Nanocube Monolayers. J. Phys. Chem. C 2012, 116 (1), 185-192. https://doi.org/10.1021/jp207682s.

(4) Faraday, M. Experimental Relations o f Gold (and Other Metals) to Light. Phil. Trans. R. Soc. 1857, 147 (0), 145-181.

(5) Kelly, K. L.; Coronado, E.; Zhao, L. L.; Schatz, G. C. The Optical Properties of Metal Nanoparticles: The Influence of Size, Shape, and Dielectric Environment. J. Phys. Chem. B 2003, 107 (3), 668-677. https://doi.org/10.1021/jp026731y.

(6) Mie, G. BeitrMge XU Optik Triiber Medden, Spexiell 7colloidaler Metallosurcgem. Ann. Der Phys. 1. 1908, 25 (37), 377-445.

(7) BARBER, D. J.; FREESTONE, I. C. An Investigation of the Origin of the Colour of the Lycurgus Cup By Analytical Transmission Electron Microscopy. Archaeometry 1990, 32 (1), 33-45. https://doi.org/10.1111/j.1475-4754.1990.tb01079.x.

(8) Maier, S. A.; Atwater, H. A. Plasmonics: Localization and Guiding of Electromagnetic Energy in Metal/Dielectric Structures. J. Appl. Phys. 2005, 98 (1). https://doi.org/10.1063/1.1951057.

(9) Jongjinakool, S.; Palasak, K.; Bousod, N.; Teepoo, S. Gold Nanoparticles-Based Colorimetric Sensor for Cysteine Detection. Energy Procedia 2014, 56 (C), 10-18. https://doi.org/10.1016/j.egypro.2014.07.126.

(10) Yu, E. T.; Derkacs, D.; Matheu, P.; Schaadt, D. M. Plasmonic Nanoparticle Scattering for Enhanced Performance of Photovoltaic and Photodetector Devices. Plasmon. Nanoimaging, Nanofabrication, Their Appl. IV 2008, 7033 (August 2008), 70331 V. https://doi.org/10.1117/12.798327.

(11) Haynes, C. L.; McFarland, A. D.; Van Duyne, R. P. Surface-Enhanced Raman Spectroscopy. Anal. Chem. 2005, 77 (17), 338 A-346 A. https://doi.org/10.1021/ac053456d.

(12) Privman, V.; Goia, D. V.; Park, J.; Matijević, E. Mechanism of Formation of Monodispersed Colloids by Aggregation of Nanosize Precursors. J. Colloid Interface Sci. 1999, 213 (1), 36-45. https://doi.org/10.1006/jcis.1999.6106.

(13) Khan, A. U.; Guo, Y.; Chen, X.; Liu, G. Spectral-Selective Plasmonic Polymer Nanocomposites Across the Visible and Near-Infrared. ACS Nano 2019, 13 (4), 4255 4266. https://doi.org/10.1021/acsnano.8b09386.

(14) Barnes, W. L. Particle Plasmons: Why Shape Matters. Am. J. Phys. 2016, 84 (8), 593-601. https://doi.org/10.1119/1.4948402.

(15) Noguez, C. Surface Plasmons on Metal Nanoparticles: The Influence of Shape and Physical Environment. J. Phys. Chem. C 2007, 111 (10), 3606-3619.

https://doi.org/10.1021/jp066539m. 
(16) Bottomley, A.; Prezgot, D.; Coyle, J. P.; Ianoul, A. Dynamics of Nanocubes Embedding into Polymer Films Investigated: Via Spatially Resolved Plasmon Modes. Nanoscale 2016, 8 (21), 11168-11176. https://doi.org/10.1039/c6nr02604d.

(17) Prezgot, D.; Ianoul, A. Optical Properties of Strongly Interacting Supported Silver Nanocube Monolayers. Plasmon. Biol. Med. X 2013, 8597 (February 2013), 85970A. https://doi.org/10.1117/12.2002568.

(18) Bottomley, A.; Ianoul, A. Reflection and Absorption Spectra of Silver Nanocubes on a Dielectric Substrate: Anisotropy, Angle, and Polarization Dependencies. J. Phys. Chem. C 2014, 118 (47), 27509-27515. https://doi.org/10.1021/jp508629d.

(19) Lal, S.; Link, S.; Halas, N. J. Nano-Optics from Sensing to Waveguiding. Nanosci. Technol. A Collect. Rev. from Nat. Journals 2009, 213-220. https://doi.org/10.1142/9789814287005_0022.

(20) Rycenga, M.; Cobley, C. M.; Zeng, J.; Li, W.; Moran, C. H.; Zhang, Q.; Qin, D.; Xia, Y. Controlling the Synthesis and Assembly of Silver Nanostructures for Plasmonic Applications. Chem. Rev. 2011, 111 (6), 3669-3712. https://doi.org/10.1021/cr100275d.

(21) Lal, S.; Link, S.; Halas, N. J. Nano-Optics From Sensing to Waveguiding. 2007, 1, 641648.

(22) Zhao, J.; Zhang, X.; Yonzon, C. R.; Hoes, A. J.; Van Duyne, R. P. Localized Surface Plasmon Resonance Biosensors. Nanomedicine 2006, 1 (2), 219-228. https://doi.org/10.2217/17435889.1.2.219.

(23) Bohren, C. F. How Can a Particle Absorb More than the Light Incident on It? Am. J. Phys. 1983, 51 (4), 323-327. https://doi.org/10.1119/1.13262.

(24) Clavero, C. Plasmon-Induced Hot-Electron Generation at Nanoparticle/Metal-Oxide Interfaces for Photovoltaic and Photocatalytic Devices. Nat. Photonics 2014, 8 (2), 95103. https://doi.org/10.1038/nphoton.2013.238.

(25) Brongersma, M. L.; Halas, N. J.; Nordlander, P. Plasmon-Induced Hot Carrier Science and Technology. Nat. Nanotechnol. 2015, 10 (1), 25-34. https://doi.org/10.1038/nnano.2014.311.

(26) Jang, Y. J.; Chung, K.; Lee, J. S.; Choi, C. H.; Lim, J. W.; Kim, D. H. Plasmonic Hot Carriers Imaging: Promise and Outlook. ACS Photonics 2018, 5 (12), 4711-4723. https://doi.org/10.1021/acsphotonics.8b01021.

(27) Mayer, K. M.; Hafner, J. H. Localized Surface Plasmon Resonance Sensors. Chem. Rev. 2011, 111 (6), 3828-3857. https://doi.org/10.1021/cr100313v.

(28) Willets, K. A.; Van Duyne, R. P. Localized Surface Plasmon Resonance Spectroscopy and Sensing. Annu. Rev. Phys. Chem. 2007, 58, 267-297.

https://doi.org/10.1146/annurev.physchem.58.032806.104607.

(29) Coronado, E. A.; Schatz, G. C. Surface Plasmon Broadening for Arbitrary Shape Nanoparticles: A Geometrical Probability Approach. J. Chem. Phys. 2003, 119 (7), 39263934. https://doi.org/10.1063/1.1587686.

(30) Malinsky, M. D.; Lance Kelly, K.; Schatz, G. C.; van Duyne, R. P. Nanosphere Lithography: Effect of Substrate on the Localized Surface Plasmon Resonance Spectrum of Silver Nanoparticles. J. Phys. Chem. B 2001, 105 (12), 2343-2350. https://doi.org/10.1021/jp002906x.

(31) Mulvaney, P. Surface Plasmon Spectroscopy of Nanosized Metal Particles. Langmuir 1996, 12 (3), 788-800. https://doi.org/10.1021/la9502711. Xiong, Y.; Washio, I.; Chen, J.; Cai, H.; Li, Z. Y.; Xia, Y. Poly (Vinyl Pyrrolidone): A 
Dual Functional Reductant and Stabilizer for the Facile Synthesis of Noble Metal Nanoplates in Aqueous Solutions. Langmuir 2006, 22 (20), 8563-8570.

https://doi.org/10.1021/la061323x.

(33) Noor, F.; Zhang, H.; Korakianitis, T.; Wen, D. Oxidation and Ignition of Aluminum Nanomaterials. Phys. Chem. Chem. Phys. 2013, 15 (46), 20176-20188. https://doi.org/10.1039/c3cp53171f.

(34) Mahmoud, M. A.; El-Sayed, M. A. Comparative Study of the Assemblies and the Resulting Plasmon Fields of Langmuir-Blodgett Assembled Monolayers of Silver Nanocubes and Gold Nanocages. J. Phys. Chem. C 2008, 112 (37), 14618-14625. https://doi.org/10.1021/jp8040499.

(35) Sönnichsen, C.; Reinhard, B. M.; Liphardt, J.; Alivisatos, A. P. A Molecular Ruler Based on Plasmon Coupling of Single Gold and Silver Nanoparticles. Nat. Biotechnol. 2005, 23 (6), 741-745. https://doi.org/10.1038/nbt1100.

(36) Blaber, M. G.; Arnold, M. D.; Ford, M. J. A Review of the Optical Properties of Alloys and Intermetallics for Plasmonics. J. Phys. Condens. Matter 2010, 22 (14). https://doi.org/10.1088/0953-8984/22/14/143201.

(37) West, P. R.; Ishii, S.; Naik, G. V.; Emani, N. K.; Shalaev, V. M.; Boltasseva, A. Searching for Better Plasmonic Materials. Laser Photonics Rev. 2010, 4 (6), 795-808. https://doi.org/10.1002/lpor.200900055.

(38) Graedel, T. E. Corrosion Mechanisms for Silver Exposed to the Atmosphere. $J$. Electrochem. Soc. 1992, 139 (7), 1963-1970. https://doi.org/10.1149/1.2221162.

(39) Lee, Y. H.; Chen, H.; Xu, Q. H.; Wang, J. Refractive Index Sensitivities of Noble Metal Nanocrystals: The Effects of Multipolar Plasmon Resonances and the Metal Type. $J$. Phys. Chem. C 2011, 115 (16), 7997-8004. https://doi.org/10.1021/jp202574r.

(40) Prezgot, D.; Bottomley, A.; Coyle, J. P.; Ianoul, A. Unusually Sharp Localized Surface Plasmon Resonance in Supported Silver Nanocrystals with a Thin Dielectric Coating. $J$. Phys. Chem. Lett. 2017, 8 (22), 5555-5558. https://doi.org/10.1021/acs.jpclett.7b02499.

(41) Meier, M.; Wokaun, A. Enhanced Fields on Large Metal Particles: Dynamic Depolarization. Opt. Lett. 1983, 8 (11), 581. https://doi.org/10.1364/ol.8.000581.

(42) Nehl, C. L.; Hafner, J. H. Shape-Dependent Plasmon Resonances of Gold Nanoparticles. J. Mater. Chem. 2008, 18 (21), 2415-2419. https://doi.org/10.1039/b714950f.

(43) Wang, Y.; Zheng, Y.; Huang, C. Z.; Xia, Y. Synthesis of Ag Nanocubes 18-32 Nm in Edge Length: The Effects of Polyol on Reduction Kinetics, Size Control, and Reproducibility. J. Am. Chem. Soc. 2013, 135 (5), 1941-1951. https://doi.org/10.1021/ja311503q.

(44) Halas, N. J.; Lal, S.; Chang, W. S.; Link, S.; Nordlander, P. Plasmons in Strongly Coupled Metallic Nanostructures. Chem. Rev. 2011, 111 (6), 3913-3961. https://doi.org/10.1021/cr200061k.

(45) Nordlander, P.; Oubre, C.; Prodan, E.; Li, K.; Stockman, M. I. Plasmon Hybridization in Nanoparticle Dimers. Nano Lett. 2004, 4 (5), 899-903. https://doi.org/10.1021/nl049681c.

(46) Romero, I.; Aizpurua, J.; Bryant, G. W.; García De Abajo, F. J. Plasmons in Nearly Touching Metallic Nanoparticles: Singular Response in the Limit of Touching Dimers. Opt. Express 2006, 14 (21), 9988. https://doi.org/10.1364/oe.14.009988.

(47) Scholl, J. A.; García-Etxarri, A.; Koh, A. L.; Dionne, J. A. Observation of Quantum Tunneling between Two Plasmonic Nanoparticles. Nano Lett. 2013, 13 (2), 564-569. https://doi.org/10.1021/nl304078v. 
(48) Esteban, R.; Borisov, A. G.; Nordlander, P.; Aizpurua, J. Bridging Quantum and Classical Plasmonics with a Quantum-Corrected Model. Nat. Commun. 2012, 3 (May). https://doi.org/10.1038/ncomms 1806 .

(49) Zuloaga, J.; Prodan, E.; Nordlander, P. Quantum Description of the Plasmon Resonances of a Nanoparticle Dimer. Nano Lett. 2009, 9 (2), 887-891. https://doi.org/10.1021/n1803811g.

(50) Savage, K. J.; Hawkeye, M. M.; Esteban, R.; Borisov, A. G.; Aizpurua, J.; Baumberg, J. J. Revealing the Quantum Regime in Tunnelling Plasmonics. Nature 2012, 491 (7425), 574577. https://doi.org/10.1038/nature11653.

(51) Su, K. H.; Wei, Q. H.; Zhang, X.; Mock, J. J.; Smith, D. R.; Schultz, S. Interparticle Coupling Effects on Plasmon Resonances of Nanogold Particles. Nano Lett. 2003, 3 (8), 1087-1090. https://doi.org/10.1021/n1034197f.

(52) Rockstuhl, C.; Salt, M. G.; Herzig, H. P. Analyzing the Scattering Properties of Coupled Metallic Nanoparticles. J. Opt. Soc. Am. A 2004, 21 (9), 1761. https://doi.org/10.1364/josaa.21.001761.

(53) Zhang, S.; Bao, K.; Halas, N. J.; Xu, H.; Nordlander, P. Substrate-Induced Fano Resonances of a Plasmonic Nanocube: A Route to Increased-Sensitivity Localized Surface Plasmon Resonance Sensors Revealed. Nano Lett. 2011, 11 (4), 1657-1663. https://doi.org/10.1021/nl200135r.

(54) Lermé, J. Plasmon Hybridization Model for a Nanoparticle above a Dielectric Interface: Dielectric Effects, Comparison with the Dimer System, Range of Applicability, and Limits. J. Phys. Chem. C 2015, 119 (36), 21087-21104. https://doi.org/10.1021/acs.jpcc.5b06129.

(55) Wu, Y.; Nordlander, P. Finite-Difference Time-Domain Modeling of the Optical Properties of Nanoparticles near Dielectric Substrates. J. Phys. Chem. C 2010, 114 (16), 7302-7307. https://doi.org/10.1021/jp908980d.

(56) Sherry, L. J.; Chang, S. H.; Schatz, G. C.; Van Duyne, R. P.; Wiley, B. J.; Xia, Y. Localized Surface Plasmon Resonance Spectroscopy of Single Silver Nanocubes. Nano Lett. 2005, 5 (10), 2034-2038. https://doi.org/10.1021/n10515753.

(57) Li, R.; Liu, K.; Pan, S.; Ding, J. H. Localized Surface Plasmon Resonance of Single Silver Nano-Hemisphere. Adv. Mater. Res. 2013, 818, 137-140. https://doi.org/10.4028/www.scientific.net/AMR.818.137.

(58) Huang, Y.; Ma, L.; Li, J.; Zhang, Z. Nanoparticle-on-Mirror Cavity Modes for Huge and/or Tunable Plasmonic Field Enhancement. Nanotechnology 2017, 28 (10). https://doi.org/10.1088/1361-6528/aa5b27.

(59) Nordlander, P.; Prodan, E. Plasmon Hybridization in Nanoparticles near Metallic Surfaces. Nano Lett. 2004, 4 (11), 2209-2213. https://doi.org/10.1021/n10486160.

(60) Le, F.; Lwin, N. Z.; Steele, J. M.; Käll, M.; Halas, N. J.; Nordlander, P. Plasmons in the Metallic Nanoparticle-Film System as a Tunable Impurity Problem. Nano Lett. 2005, 5 (10), 2009-2013. https://doi.org/10.1021/n10515100.

(61) Moularas, C.; Georgiou, Y.; Adamska, K.; Deligiannakis, Y. Thermoplasmonic Heat Generation Efficiency by Nonmonodisperse Core-Shell Ag0@SiO2 Nanoparticle Ensemble. J. Phys. Chem. C 2019, 123 (36), 22499-22510. https://doi.org/10.1021/acs.jpcc.9b06532.

(62) Baffou, G.; Rigneault, H. Femtosecond-Pulsed Optical Heating of Gold Nanoparticles. Phys. Rev. B - Condens. Matter Mater. Phys. 2011, 84 (3), 1-13. 
https://doi.org/10.1103/PhysRevB.84.035415.

(63) Baffou, G.; Quidant, R.; García De Abajo, F. J. Nanoscale Control of Optical Heating in Complex Plasmonic Systems. ACS Nano 2010, 4 (2), 709-716. https://doi.org/10.1021/nn901144d.

(64) Besteiro, L. V.; Kong, X. T.; Wang, Z.; Hartland, G.; Govorov, A. O. Understanding HotElectron Generation and Plasmon Relaxation in Metal Nanocrystals: Quantum and Classical Mechanisms. ACS Photonics 2017, 4 (11), 2759-2781. https://doi.org/10.1021/acsphotonics.7b00751. Magnozzi, M.; Proietti Zaccaria, R.; Catone, D.; O’Keeffe, P.; Paladini, A.; Toschi, F.; Alabastri, A.; Canepa, M.; Bisio, F. Interband Transitions Are More Efficient Than Plasmonic Excitation in the Ultrafast Melting of Electromagnetically Coupled $\mathrm{Au}$ Nanoparticles. J. Phys. Chem. C 2019, 123 (27), 16943-16950. https://doi.org/10.1021/acs.jpcc.9b03668.

(66) Baffou, G.; Quidant, R. Thermo-Plasmonics: Using Metallic Nanostructures as NanoSources of Heat. Laser Photonics Rev. 2013, 7 (2), 171-187. https://doi.org/10.1002/lpor.201200003.

(67) Zhu, X.; Vannahme, C.; Højlund-Nielsen, E.; Mortensen, N. A.; Kristensen, A. Plasmonic Colour Laser Printing. Nat. Nanotechnol. 2016, 11 (4), 325-329. https://doi.org/10.1038/nnano.2015.285.

(68) Baffou, G.; Cichos, F.; Quidant, R. Applications and Challenges of Thermoplasmonics. Nat. Mater. 2020, 19 (9), 946-958. https://doi.org/10.1038/s41563-020-0740-6.

(69) Baffou, G.; Berto, P.; Bermúdez Ureña, E.; Quidant, R.; Monneret, S.; Polleux, J.; Rigneault, H. Photoinduced Heating of Nanoparticle Arrays. ACS Nano 2013, 7 (8), 6478-6488. https://doi.org/10.1021/nn401924n.

(70) Jauffred, L.; Samadi, A.; Klingberg, H.; Bendix, P. M.; Oddershede, L. B. Plasmonic Heating of Nanostructures. Chem. Rev. 2019, 119 (13), 8087-8130. https://doi.org/10.1021/acs.chemrev.8b00738.

(71) Chien, M. H.; Brameshuber, M.; Rossboth, B. K.; Schütz, G. J.; Schmid, S. SingleMolecule Optical Absorption Imaging by Nanomechanical Photothermal Sensing. Proc. Natl. Acad. Sci. U. S. A. 2018, 115 (44), 11150-11155. https://doi.org/10.1073/pnas.1804174115.

(72) Lalisse, A.; Tessier, G.; Plain, J.; Baffou, G. Quantifying the Efficiency of Plasmonic Materials for Near-Field Enhancement and Photothermal Conversion. J. Phys. Chem. C 2015, 119 (45), 25518-25528. https://doi.org/10.1021/acs.jpcc.5b09294.

(73) Herzog, J. B.; Knight, M. W.; Li, Y.; Evans, K. M.; Halas, N. J.; Natelson, D. Dark Plasmons in Hot Spot Generation and Polarization in Interelectrode Nanoscale Junctions. Nano Lett. 2013, 13 (3), 1359-1364. https://doi.org/10.1021/nl400363d.

(74) Baffou, G.; Quidant, R.; Girard, C. Thermoplasmonics Modeling: A Green's Function Approach. Phys. Rev. B - Condens. Matter Mater. Phys. 2010, 82 (16), 1-11. https://doi.org/10.1103/PhysRevB.82.165424.

(75) Rangacharya, V. P.; Wu, K.; Larsen, P. E.; Thamdrup, L. H. E.; Ilchenko, O.; Hwu, E. Te; Rindzevicius, T.; Boisen, A. Quantifying Optical Absorption of Single Plasmonic Nanoparticles and Nanoparticle Dimers Using Microstring Resonators. ACS Sensors 2020, 5 (7), 2067-2075. https://doi.org/10.1021/acssensors.0c00591.

(76) Pillai, Z. S.; Kamat, P. V. What Factors Control the Size and Shape of Silver Nanoparticles in the Citrate Ion Reduction Method? J. Phys. Chem. B 2004, 108 (3), 945- 
951. https://doi.org/10.1021/jp037018r.

(77) Bastús, N. G.; Comenge, J.; Puntes, V. Kinetically Controlled Seeded Growth Synthesis of Citrate-Stabilized Gold Nanoparticles of up to $200 \mathrm{Nm}$ : Size Focusing versus Ostwald Ripening. Langmuir 2011, 27 (17), 11098-11105. https://doi.org/10.1021/la201938u.

(78) Lv, L.; Jin, Y.; Kang, X.; Zhao, Y.; Cui, C.; Guo, Z. PVP-Coated Gold Nanoparticles for the Selective Determination of Ochratoxin A via Quenching Fluorescence of the Free Aptamer. Food Chem. 2018, 249 (July 2017), 45-50. https://doi.org/10.1016/j.foodchem.2017.12.087.

(79) Wiley, B.; Sun, Y.; Xia, Y. Synthesis of Silver Nanostructures with Controlled Shapes and Properties. Acc. Chem. Res. 2007, 40 (10), 1067-1076. https://doi.org/10.1021/ar7000974.

(80) Skrabalak, S. E.; Wiley, B. J.; Kim, M.; Formo, E. V.; Xia, Y. On the Polyol Synthesis of Silver Nanostructures: Glycolaldehyde as a Reducing Agent. Nano Lett. 2008, 8 (7), 2077-2081. https://doi.org/10.1021/nl800910d.

(81) Sun, Y. Controlled Synthesis of Colloidal Silver Nanoparticles in Organic Solutions: Empirical Rules for Nucleation Engineering. Chem. Soc. Rev. 2013, 42 (7), 2497-2511. https://doi.org/10.1039/c2cs35289c.

(82) LaMer, V. K.; Dinegar, R. H. Theory, Production and Mechanism of Formation of Monodispersed Hydrosols. J. Am. Chem. Soc. 1950, 72 (11), 4847-4854. https://doi.org/10.1021/ja01167a001.

(83) Gommes, C. J. Ostwald Ripening of Confined Nanoparticles: Chemomechanical Coupling in Nanopores. Nanoscale 2019, 11 (15), 7386-7393. https://doi.org/10.1039/c9nr01349k.

(84) Kuo, C. L.; Hwang, K. C. Does Morphology of a Metal Nanoparticle Play a Role in Ostwald Ripening Processes? Chem. Mater. 2013, 25 (3), 365-371. https://doi.org/10.1021/cm3031279.

(85) Kryukov, A. I.; Zin'Chuk, N. N.; Korzhak, A. V.; Kuchmii, Y. S. Catalysis of the Reduction of Silver Ions by AG2s Nanoparticles. Theor. Exp. Chem. 2001, 37 (6), 355359. https://doi.org/10.1023/A:1014704315241.

(86) Wang, Z.; Ma, L. Gold Nanoparticle Probes. Coord. Chem. Rev. 2009, 253 (11-12), 1607-1618. https://doi.org/10.1016/j.ccr.2009.01.005.

(87) MA, L. N.; LIU, D. J.; WANG, Z. X. Synthesis and Applications of Gold Nanoparticle Probes. Chinese J. Anal. Chem. 2010, 38 (1), 1-7. https://doi.org/10.1016/S18722040(09)60013-X.

(88) Alba-Molina, D.; Puente Santiago, A. R.; Giner-Casares, J. J.; Martín-Romero, M. T.; Camacho, L.; Luque, R.; Cano, M. Citrate-Stabilized Gold Nanoparticles as HighPerformance Electrocatalysts: The Role of Size in the Electroreduction of Oxygen. $J$. Phys. Chem. C 2019, 123 (15), 9807-9812. https://doi.org/10.1021/acs.jpcc.9b00249.

(89) Kang, H.; Buchman, J. T.; Rodriguez, R. S.; Ring, H. L.; He, J.; Bantz, K. C.; Haynes, C. L. Stabilization of Silver and Gold Nanoparticles: Preservation and Improvement of Plasmonic Functionalities. Chem. Rev. 2019, 119 (1), 664-699. https://doi.org/10.1021/acs.chemrev.8b00341.

(90) Vu, X. H.; Duong, T. T. T.; Pham, T. T. H.; Trinh, D. K.; Nguyen, X. H.; Dang, V. S. Synthesis and Study of Silver Nanoparticles for Antibacterial Activity against Escherichia Coli and Staphylococcus Aureus. Adv. Nat. Sci. Nanosci. Nanotechnol. 2018, 9 (2). https://doi.org/10.1088/2043-6254/aac58f.

(91) Bastús, N. G.; Merkoçi, F.; Piella, J.; Puntes, V. Synthesis of Highly Monodisperse Citrate-Stabilized Silver Nanoparticles of up to $200 \mathrm{Nm}$ : Kinetic Control and Catalytic 
Properties. Chem. Mater. 2014, 26 (9), 2836-2846. https://doi.org/10.1021/cm500316k.

(92) Ahn, H. Y.; Lee, H. E.; Jin, K.; Nam, K. T. Extended Gold Nano-Morphology Diagram: Synthesis of Rhombic Dodecahedra Using CTAB and Ascorbic Acid. J. Mater. Chem. C 2013, 1 (41), 6861-6868. https://doi.org/10.1039/c3tc31135j.

(93) Niu, W.; Zhang, L.; Xu, G. Seed-Mediated Growth of Noble Metal Nanocrystals: Crystal Growth and Shape Control. Nanoscale 2013, 5 (8), 3172-3181.

https://doi.org/10.1039/c3nr00219e.

(94) Zhao, L.; Jiang, D.; Cai, Y.; Ji, X.; Xie, R.; Yang, W. Tuning the Size of Gold Nanoparticles in the Citrate Reduction by Chloride Ions. Nanoscale 2012, 4 (16), 50715076. https://doi.org/10.1039/c2nr30957b.

(95) Xia, H.; Bai, S.; Hartmann, J.; Wang, D. Synthesis of Monodisperse Quasi-Spherical Gold Nanoparticles in Water via Silver(I)-Assisted Citrate Reduction. Langmuir 2010, 26 (5), 3585-3589. https://doi.org/10.1021/la902987w.

(96) Matijević, E. Nanosize Precursors as Building Blocks for Monodispersed Colloids. Colloid J. 2007, 69 (1), 29-38. https://doi.org/10.1134/S1061933X0701005X.

(97) Ji, X.; Song, X.; Li, J.; Bai, Y.; Yang, W.; Peng, X. Size Control of Gold Nanocrystals in Citrate Reduction: The Third Role of Citrate. J. Am. Chem. Soc. 2007, 129 (45), 1393913948. https://doi.org/10.1021/ja074447k.

(98) Daniel, M. C.; Astruc, D. Gold Nanoparticles: Assembly, Supramolecular Chemistry, Quantum-Size-Related Properties, and Applications Toward Biology, Catalysis, and Nanotechnology. Chem. Rev. 2004, 104 (1), 293-346. https://doi.org/10.1021/cr030698+.

(99) Fan, Q.; Liu, K.; Liu, Z.; Liu, H.; Zhang, L.; Zhong, P.; Gao, C. A Ligand-Exchange Route to Nobel Metal Nanocrystals with a Clean Surface for Enhanced Optical and Catalytic Properties. Part. Part. Syst. Charact. 2017, 34 (8), 1-8. https://doi.org/10.1002/ppsc.201700075.

(100) Woehrle, G. H.; Brown, L. O.; Hutchison, J. E. Thiol-Functionalized, 1.5-Nm Gold Nanoparticles through Ligand Exchange Reactions: Scope and Mechanism of Ligand Exchange. J. Am. Chem. Soc. 2005, 127 (7), 2172-2183. https://doi.org/10.1021/ja0457718.

(101) Dong, A.; Ye, X.; Chen, J.; Kang, Y.; Gordon, T.; Kikkawa, J. M.; Murray, C. B. A Generalized Ligand-Exchange Strategy Enabling Sequential Surface Functionalization of Colloidal Nanocrystals. J. Am. Chem. Soc. 2011, 133 (4), 998-1006. https://doi.org/10.1021/jal08948z.

(102) Overney, R. M.; Buenviaje, C.; Luginbühl, R.; Dinelli, F. Glass and Structural Transitions Measured at Polymer Surfaces on the Nanoscale. J. Therm. Anal. Calorim. 2000, 59 (1), 205-225. https://doi.org/10.1023/A:1010196214867.

(103) Ellison, C. J.; Torkelson, J. M. The Distribution of Glass-Transition Temperatures in Nanoscopically Confined Glass Formers. Nat. Mater. 2003, 2 (10), 695-700. https://doi.org/10.1038/nmat980.

(104) Vignaud, G.; Chebil, M. S.; Bal, J. K.; Delorme, N.; Beuvier, T.; Grohens, Y.; Gibaud, A. Densification and Depression in Glass Transition Temperature in Polystyrene Thin Films. Langmuir 2014, 30 (39), 11599-11608. https://doi.org/10.1021/la501639z.

(105) Tsui, O. K. C.; Russell, T. P.; Hawker, C. J. Effect of Polymer-Substrate Interactions on the Glass Transition of Polymer Thin Films. AIP Conf. Proc. 2004, 708, 598-600. https://doi.org/10.1063/1.1764232.

(106) Ediger, M. D.; Forrest, J. A. Dynamics near Free Surfaces and the Glass Transition in 
Thin Polymer Films: A View to the Future. Macromolecules 2014, 47 (2), 471-478. https://doi.org/10.1021/ma4017696.

(107) Zhang, Y.; Jeong, C. K.; Wang, J.; Sun, H.; Li, F.; Zhang, G.; Chen, L. Q.; Zhang, S.; Chen, W.; Wang, Q. Flexible Energy Harvesting Polymer Composites Based on BiofibrilTemplated 3-Dimensional Interconnected Piezoceramics. Nano Energy 2018, 50 (May), 35-42. https://doi.org/10.1016/j.nanoen.2018.05.025.

(108) Ferhan, A. R.; Kim, D. H. Nanoparticle Polymer Composites on Solid Substrates for Plasmonic Sensing Applications. Nano Today 2016, 11 (4), 415-434. https://doi.org/10.1016/j.nantod.2016.07.001.

(109) Bushell, M.; Ianoul, A. Interfacial Diffusion of Silver Nanocrystals into Polymer Surfaces Monitored by Hybrid Plasmon Modes. J. Phys. Chem. C 2018, 122 (18), 10197-10204. https://doi.org/10.1021/acs.jpcc.8b00314.

(110) Shimomura, S.; Inutsuka, M.; Tajima, K.; Nabika, M.; Moritomi, S.; Matsuno, H.; Tanaka, K. Stabilization of Polystyrene Thin Films by Introduction of a Functional End Group. Polym. J. 2016, 48 (9), 949-953. https://doi.org/10.1038/pj.2016.58.

(111) Acharya, S.; Hill, J. P.; Ariga, K. Soft Langmuir-Blodgett Technique for Hard Nanomaterials. Adv. Mater. 2009, 21 (29), 2959-2981. https://doi.org/10.1002/adma.200802648.

(112) Lu, M.; Zhu, H.; Bazuin, C. G.; Peng, W.; Masson, J. F. Polymer-Templated Gold Nanoparticles on Optical Fibers for Enhanced-Sensitivity Localized Surface Plasmon Resonance Biosensors. ACS Sensors 2019, 4 (3), 613-622. https://doi.org/10.1021/acssensors.8b01372.

(113) Langmuir Blodgett Troughs Operating Manual, 6th ed.; Nima, 2002.

(114) Fainerman, V. B.; Vollhardt, D. Equations of State for Langmuir Monolayers with TwoDimensional Phase Transitions. J. Phys. Chem. B 1999, 103 (1), 145-150. https://doi.org/10.1021/jp983109q.

(115) Rubinger, C. P. L.; Moreira, R. L.; Cury, L. A.; Fontes, G. N.; Neves, B. R. A.; Meneguzzi, A.; Ferreira, C. A. Langmuir-Blodgett and Langmuir-Schaefer Films of Poly(5-Amino-1-Naphthol) Conjugated Polymer. Appl. Surf. Sci. 2006, 253 (2), 543-548. https://doi.org/10.1016/j.apsusc.2005.12.096.

(116) Kovacs, G. J.; Vincett, P. S. Formation and Thermodynamic Stability of a Novel Class of Useful Materials: Close-Packed Monolayers of Submicron Monodisperse Spheres Just below a Polymer Surface. J. Colloid Interface Sci. 1982, 90 (2), 335-351. https://doi.org/10.1016/0021-9797(82)90302-2.

(117) Sullivan, D. M. ELECTROMAGNETIC SIMULATION USING THE FDTD METHOD; A JOHN WILEY \&SONS, INC., PUBLICATION, 2000.

(118) Zhao, J.; Pinchuk, A. O.; McMahon, J. M.; Li, S.; Ausman, L. K.; Atkinson, A. L.; Schatz, G. C. Methods for Describing the Electromagnetic Properties of Silver and Gold Nanoparticles. Acc. Chem. Res. 2008, 41 (12), 1710-1720. https://doi.org/10.1021/ar800028j.

(119) Khoury, C. G.; Norton, S. J.; Vo-Dinh, T. Investigating the Plasmonics of a DipoleExcited Silver Nanoshell: Mie Theory versus Finite Element Method. Nanotechnology 2010, 21 (31). https://doi.org/10.1088/0957-4484/21/31/315203.

(120) Draine, B. T.; Flatau, P. J. Discrete-Dipole Approximation For Scattering Calculations. J. Opt. Soc. Am. A 1994, 11 (4), 1491. https://doi.org/10.1364/josaa.11.001491.

(121) Gray, S. K. Theory and Modeling of Plasmonic Structures. J. Phys. Chem. C 2013, 117 
(5), 1983-1994. https://doi.org/10.1021/jp309664c.

(122) Montgomery, J. M.; Lee, T. W.; Gray, S. K. Theory and Modeling of Light Interactions with Metallic Nanostructures. J. Phys. Condens. Matter 2008, 20 (32). https://doi.org/10.1088/0953-8984/20/32/323201.

(123) Yee, K. S. Numerical Solution of Initial Boundary Value Problems Involving Maxwell's Equations in Isotropic Media. IEEE Trans. Antennas Propag. 1966, 14 (3), 302-307. https://doi.org/10.1109/TAP.1966.1138693.

(124) Choroszucho, A. Analysis of the Influence of the Complex Structure of Clay Hollow Bricks on the Values of Electric Field Intensity by Using the FDTD Method. Arch. Electr. Eng. 2016, 65 (4), 745-759. https://doi.org/10.1515/aee-2016-0052.

(125) Gedney, S. D. Introduction to the Finite-Difference Time-Domain (FDTD)) Method for Electromagnetics; 2011; Vol. 27. https://doi.org/10.2200/S00316ED1V01Y201012CEM027.

(126) Mercatelli, R.; Romano, G.; Ratto, F.; Matteini, P.; Centi, S.; Cialdai, F.; Monici, M.; Pini, R.; Fusi, F. Quantitative Measurement of Scattering and Extinction Spectra of Nanoparticles by Darkfield Microscopy. Appl. Phys. Lett. 2011, 99 (13), 1-4. https://doi.org/10.1063/1.3636439.

(127) Grand, J.; Auguié, B.; Le Ru, E. C. Combined Extinction and Absorption UV-Visible Spectroscopy as a Method for Revealing Shape Imperfections of Metallic Nanoparticles. Anal. Chem. 2019, 91 (22), 14639-14648. https://doi.org/10.1021/acs.analchem.9b03798.

(128) Movsesyan, A.; Baudrion, A.-L.; Adam, P.-M. Extinction Measurements of Metallic Nanoparticles Arrays as a Way to Explore the Single Nanoparticle Plasmon Resonances. Opt. Express 2018, 26 (5), 6439. https://doi.org/10.1364/oe.26.006439.

(129) Near, R.; Hayden, S.; El-Sayed, M. Extinction vs Absorption: Which Is the Indicator of Plasmonic Field Strength for Silver Nanocubes? J. Phys. Chem. C 2012, 116 (43), 23019 23026. https://doi.org/10.1021/jp309272b.

(130) Chen, H.; Sun, Z.; Ni, W.; Woo, K. C.; Lin, H. Q.; Sun, L.; Yan, C.; Wang, J. Plasmon Coupling in Clusters Composed of Two-Dimensionally Ordered Gold Nanocubes. Small 2009, 5 (18), 2111-2119. https://doi.org/10.1002/smll.200900256.

(131) Shaw, M.; Fairchild, M. Evaluating the 1931 CIE Color-Matching Functions. Color Res. Appl. 2002, 27 (5), 316-329. https://doi.org/10.1002/col.10077.

(132) Fairman, H. S.; Brill, M. H.; Hemmendinger, H. How the CIE 1931 Color-Matching Functions Were Derived from Wright-Guild Data. Color Res. Appl. 1997, 22 (1), 11-23. https://doi.org/10.1002/(SICI)1520-6378(199702)22:1<11::AID-COL4>3.0.CO;2-7.

(133) Bottomley, A.; Prezgot, D.; Staff, A.; Ianoul, A. Fine Tuning of Plasmonic Properties of Monolayers of Weakly Interacting Silver Nanocubes on Thin Silicon Films. Nanoscale 2012, 4 (20), 6374-6382. https://doi.org/10.1039/c2nr31885g.

(134) Bushell, M.; Ianoul, A. Selective Embedment of Silver Nanocrystals into Spatially Segregated Domains in Thin Polymer Films for Controlled Fabrication of Functional Nanocomposites. RSC Adv. 2019, 9 (33), 19131-19141. https://doi.org/10.1039/c9ra02490e.

(135) Naguib, Y. W.; Cui, Z. Nanomedicine: The Promise and Challenges in Cancer Chemotherapy; 2014; Vol. 811. https://doi.org/10.1007/978-94-017-8739-0_11.

(136) Chegel, V.; Rachkov, O.; Lopatynskyi, A.; Ishihara, S.; Yanchuk, I.; Nemoto, Y.; Hill, J. P.; Ariga, K. Gold Nanoparticles Aggregation: Drastic Effect of Cooperative Functionalities in a Single Molecular Conjugate. J. Phys. Chem. C 2012, 116 (4), 2683- 
2690. https://doi.org/10.1021/jp209251y.

(137) Yang, Y.; Hu, Y.; Du, H.; Wang, H. Intracellular Gold Nanoparticle Aggregation and Their Potential Applications in Photodynamic Therapy. Chem. Commun. 2014, 50 (55), 7287-7290. https://doi.org/10.1039/c4cc02376e.

(138) Lothian, G. F. Beer' s Law and Its Use in Analysis. Analyst 1852, 88 (678), 678-685.

(139) Kirkland, E. Selective Thermoplasmonic Embedment of Supported Silver Nanocrystals Selective Thermoplasmonic Embedment of Supported Silver Nanocrystals Submitted By : 2020.

(140) Håkanson, U.; Agio, M.; Kühn, S.; Rogobete, L.; Kalkbrenner, T.; Sandoghdar, V. Coupling of Plasmonic Nanoparticles to Their Environments in the Context of van Der Waals-Casimir Interactions. Phys. Rev. B - Condens. Matter Mater. Phys. 2008, 77 (15). https://doi.org/10.1103/PhysRevB.77.155408.

(141) Mencarelli, E.; Fanò, L.; Tarpani, L.; Latterini, L. Modelling the Optical Properties of Metal Nanoparticles: Analytical vs Finite Elements Simulation. Mater. Today Proc. 2015, 2 (1), 161-170. https://doi.org/10.1016/j.matpr.2015.04.062.

(142) Robert, H. M. L.; Kundrat, F.; Bermúdez-Ureña, E.; Rigneault, H.; Monneret, S.; Quidant, R.; Polleux, J.; Baffou, G. Light-Assisted Solvothermal Chemistry Using Plasmonic Nanoparticles. ACS Omega 2016, 1 (1), 2-8. https://doi.org/10.1021/acsomega.6b00019.

(143) Huang, J.; Traverso, A. J.; Yang, G.; Mikkelsen, M. H. Real-Time Tunable Strong Coupling: From Individual Nanocavities to Metasurfaces. ACS Photonics 2019, 6 (4), 838-843. https://doi.org/10.1021/acsphotonics.8b01743.

(144) Wang, H.; Brandl, D. W.; Le, F.; Nordlander, P.; Halas, N. J. Nanorice: A Hybrid Plasmonic Nanostructure. Nano Lett. 2006, 6 (4), 827-832. https://doi.org/10.1021/n1060209w.

(145) Schertz, F.; Schmelzeisen, M.; Mohammadi, R.; Kreiter, M.; Elmers, H. J.; Schönhense, G. Near Field of Strongly Coupled Plasmons: Uncovering Dark Modes. Nano Lett. 2012, 12 (4), 1885-1890. https://doi.org/10.1021/nl204277y.

(146) Baumberg, J. J.; Aizpurua, J.; Mikkelsen, M. H.; Smith, D. R. Extreme Nanophotonics from Ultrathin Metallic Gaps. Nat. Mater. 2019, 18 (7), 668-678. https://doi.org/10.1038/s41563-019-0290-y.

(147) Prezgot, D. Functional Properties of Hybrid Resonances in Plasmonic Nanocrystals by Daniel Prezgot A Thesis Submitted to the Faculty of Graduate and Postdoctoral Affairs in Partial Fulfillment of the Requirements for the Degree of Doctor of Philosophy In. 2020. 


\section{Appendix:}
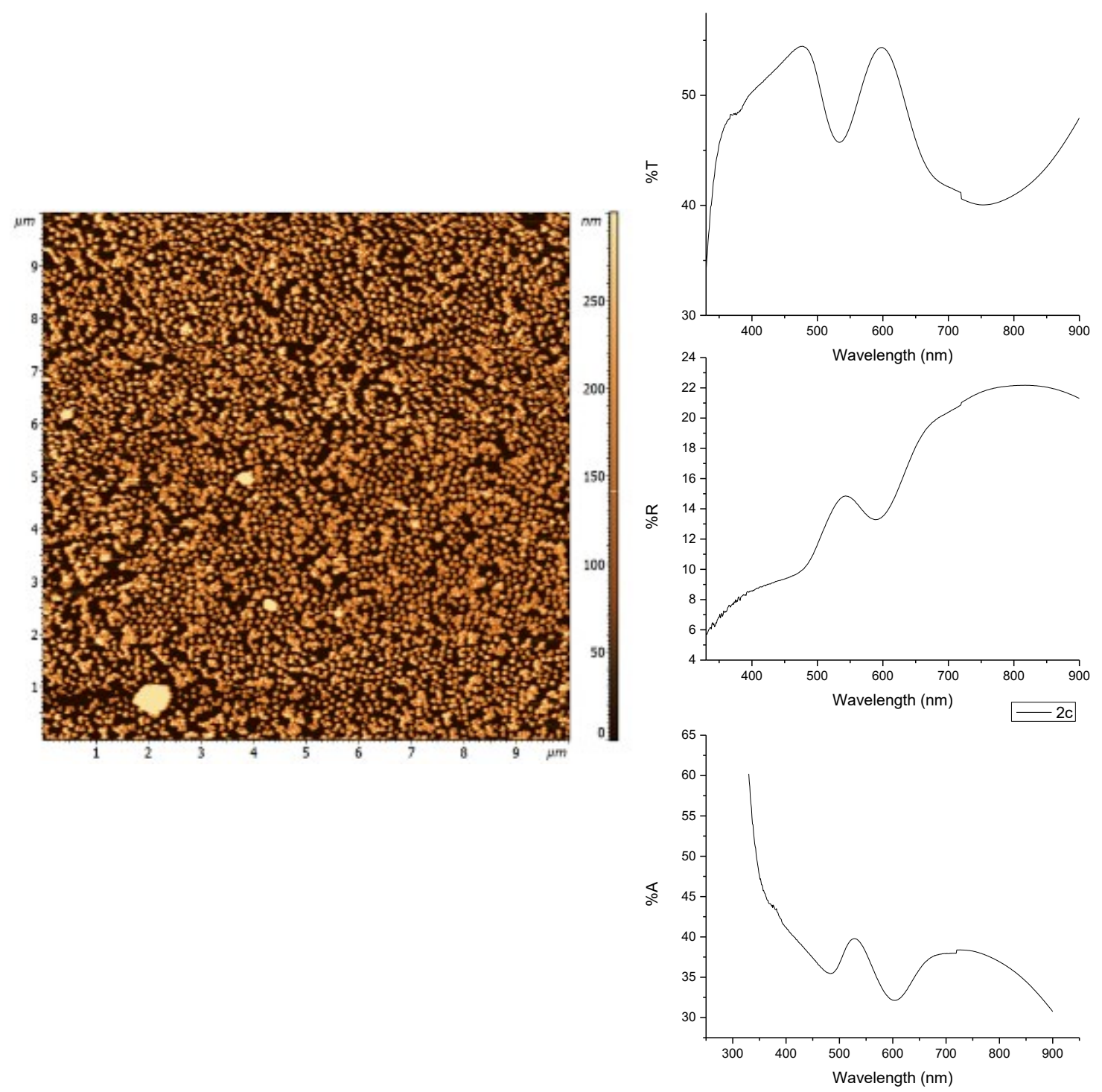

Figure 48: $2 \mathrm{mN} / \mathrm{m}$ AuNPs on PS. AFM image (left) and the T, R, A spectra (right) 

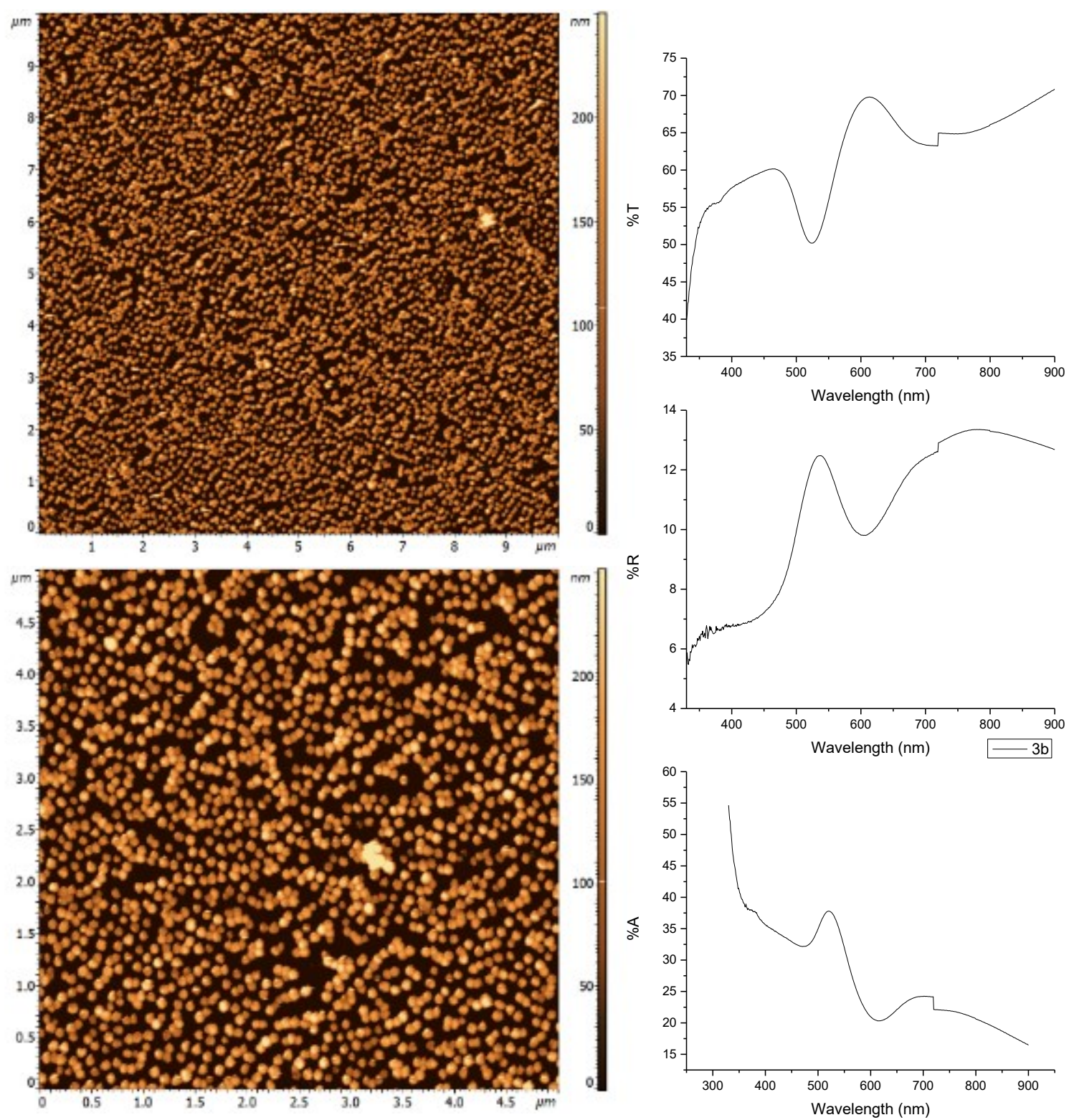

Figure 49: $3 \mathrm{mN} / \mathrm{m}$ AuNPs on PS. AFM images (left) $10 \times 10 \mu \mathrm{m}^{2}$ (top) and $5 \times 5 \mu \mathrm{m}^{2}$ (bottom), and the T, $\mathrm{R}$, A spectra (right) 

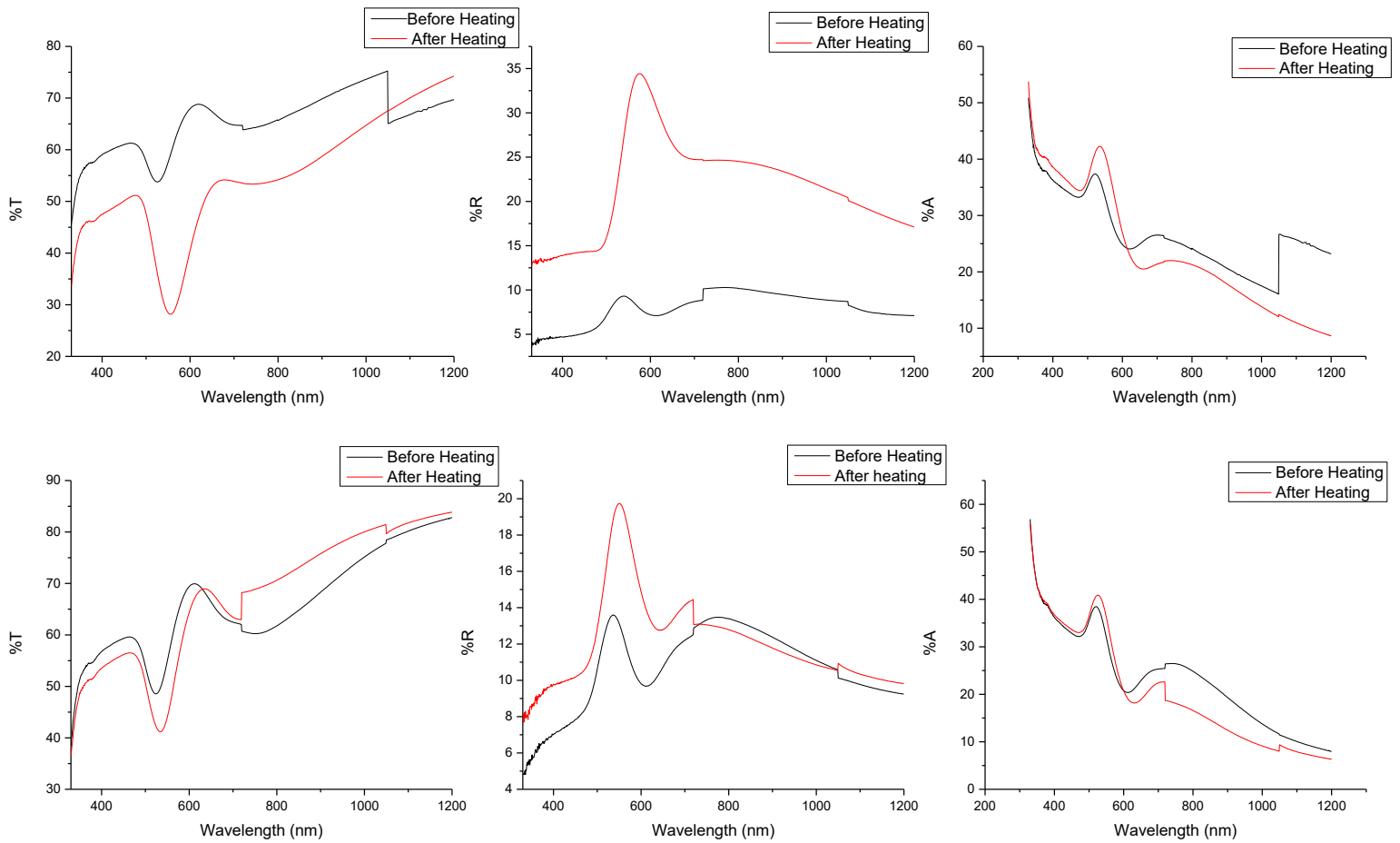

Figure 50: T, R, A spectra for $2 \mathrm{mN} / \mathrm{m}$ (top) and $3 \mathrm{mN} / \mathrm{m}$ (bottom) AuNPs on PS before and after heating.
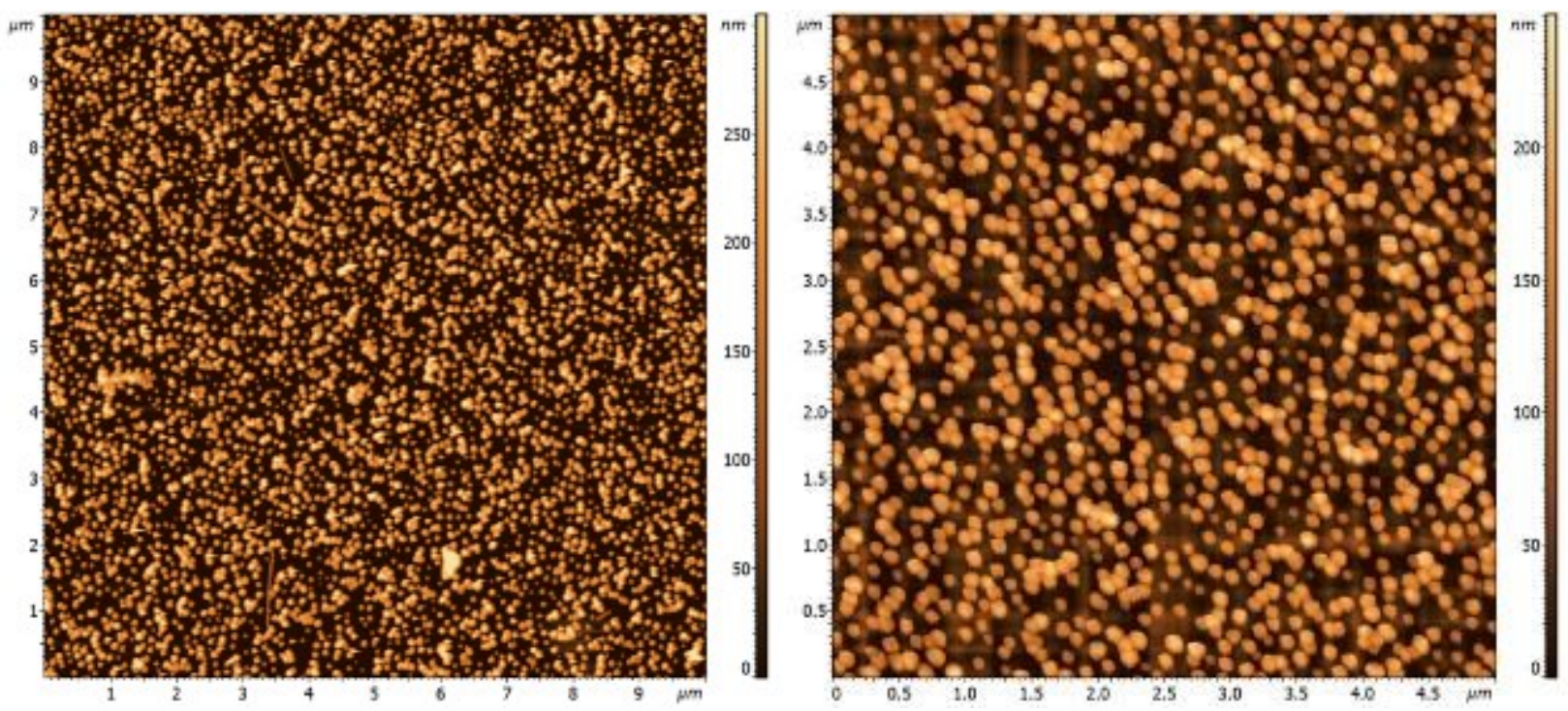

Figure 51: AFM images of a $1 \mathrm{mN} / \mathrm{m}$ mix on polystyrene, $10 \mathrm{x} 10 \mu \mathrm{m}^{2}$ (left) and $5 \times 5 \mu \mathrm{m}^{2}$ (right) 

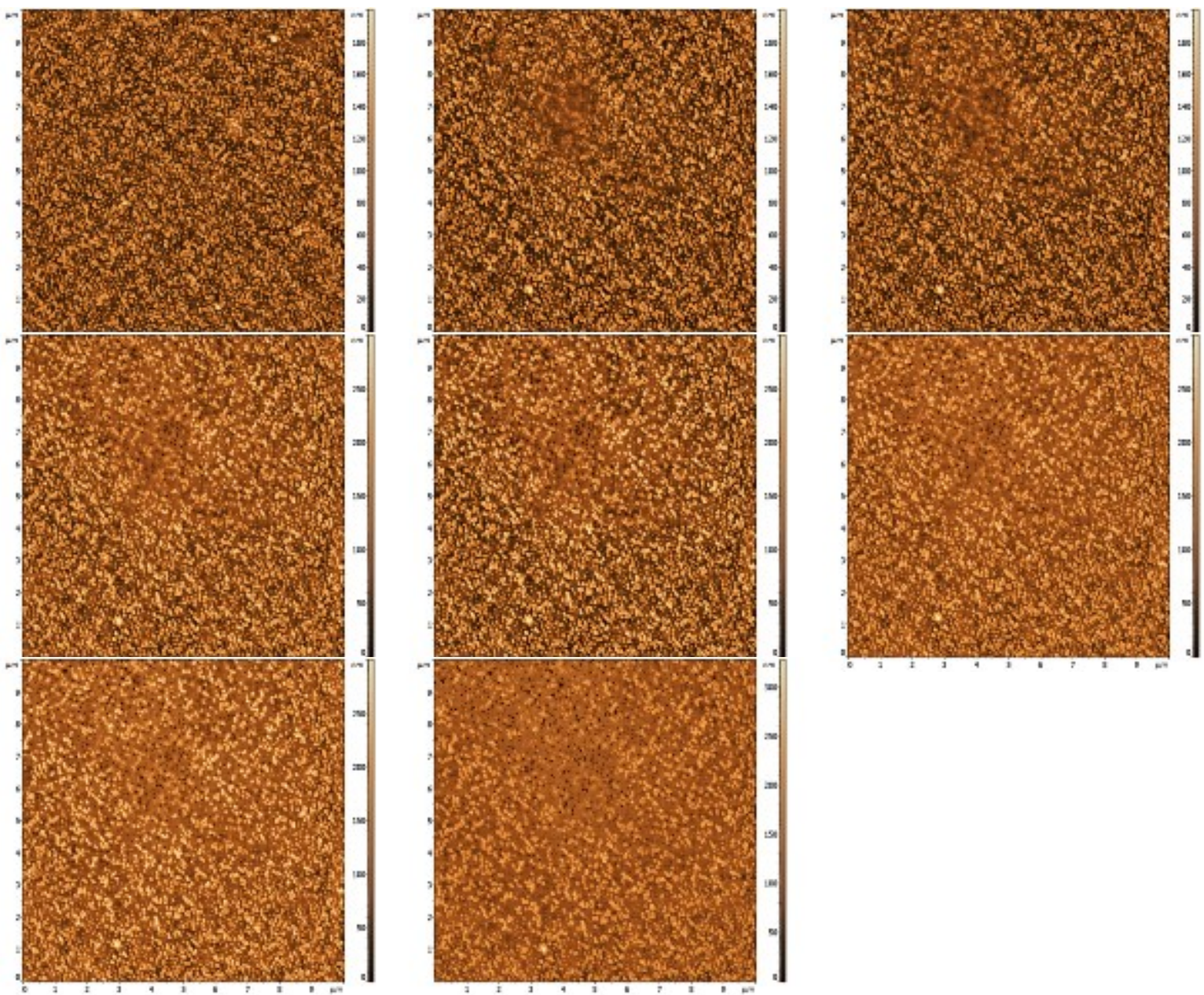

Figure 52: Selective Embedment on 1:1 mix at $458 \mathrm{~nm}$. In order the images represent $0 \mathrm{~s}, 5 \mathrm{~s}, 15 \mathrm{~s}, 25 \mathrm{~s}$, $35 \mathrm{~s}, 55 \mathrm{~s}, 2 \mathrm{~min}$, and $7 \mathrm{~min}$ of laser exposure. 

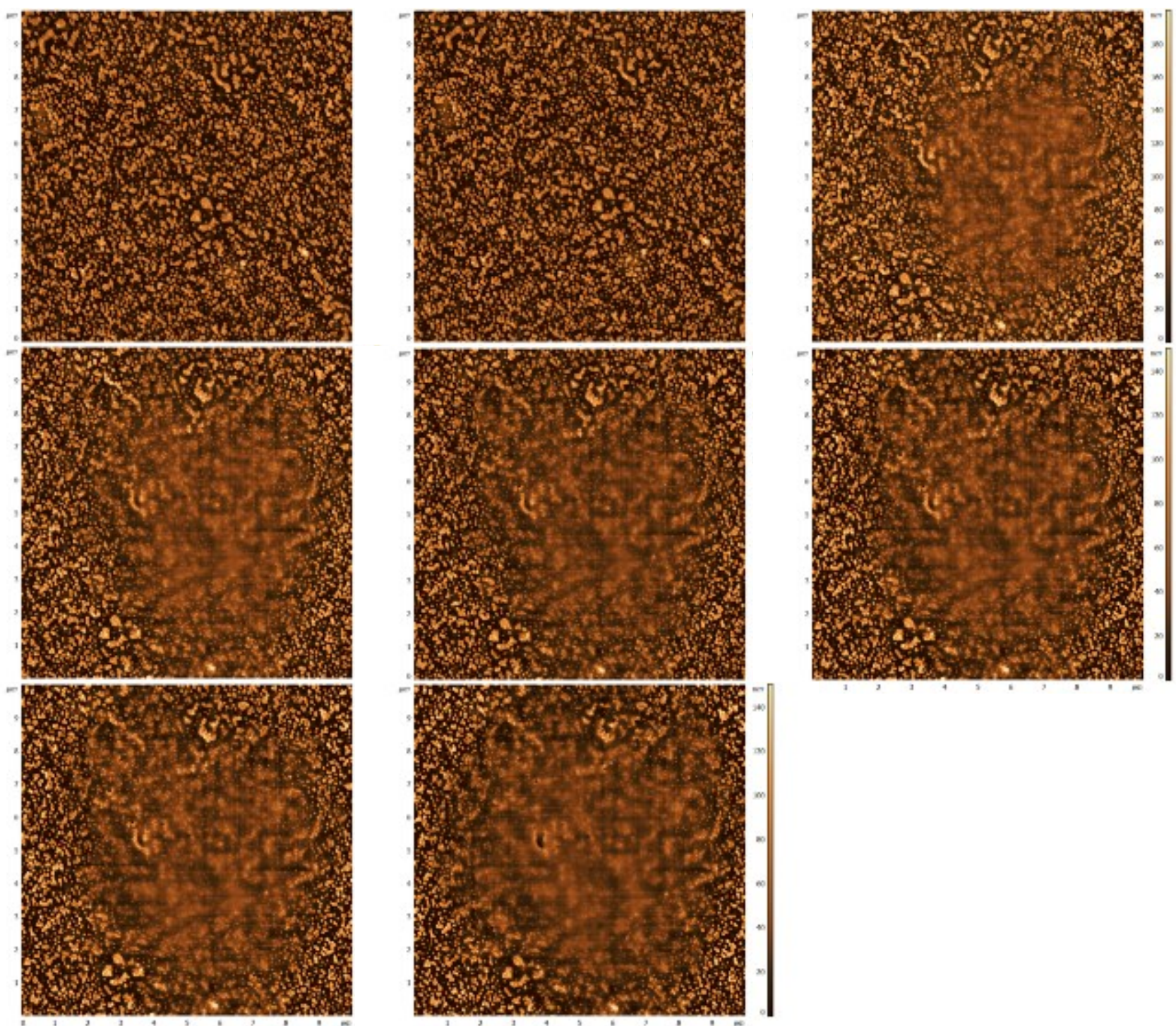

Figure 53: Selective Embedment on 1:1 mix at $568 \mathrm{~nm}$. In order the images represent $0 \mathrm{~s}, 5 \mathrm{~s}, 35 \mathrm{~s}, 50 \mathrm{~s}$, $65 \mathrm{~s}, 1 \mathrm{~min} 35 \mathrm{~s}, 3 \mathrm{~min}$, and $6 \mathrm{~min}$ of laser exposure. 

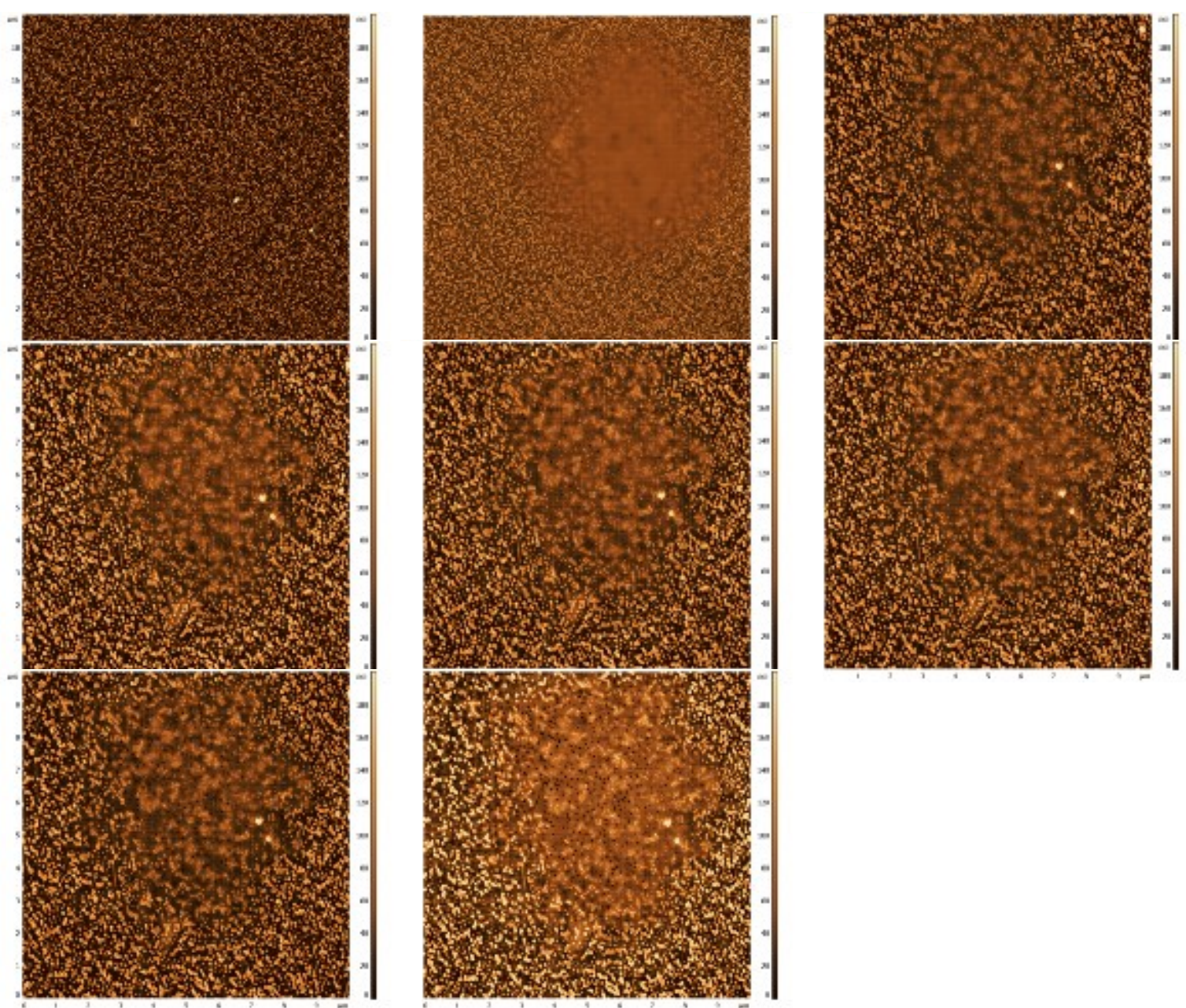

Figure 54: Selective Embedment on 1:1 mix at $568 \mathrm{~nm}$ then $458 \mathrm{~nm}$. Firstly, the substrate was exposed to $568 \mathrm{~nm}$ for $10 \mathrm{~s}(40 \mathrm{~mW})$, then another $10 \mathrm{~s}(50 \mathrm{~mW})$. The laser was switched to 458 and the substrate was exposed for $5 \mathrm{~s}, 10 \mathrm{~s}, 15 \mathrm{~s}, 30 \mathrm{~s}$, and $1 \mathrm{~min}$. The images are shown in chronological order. 

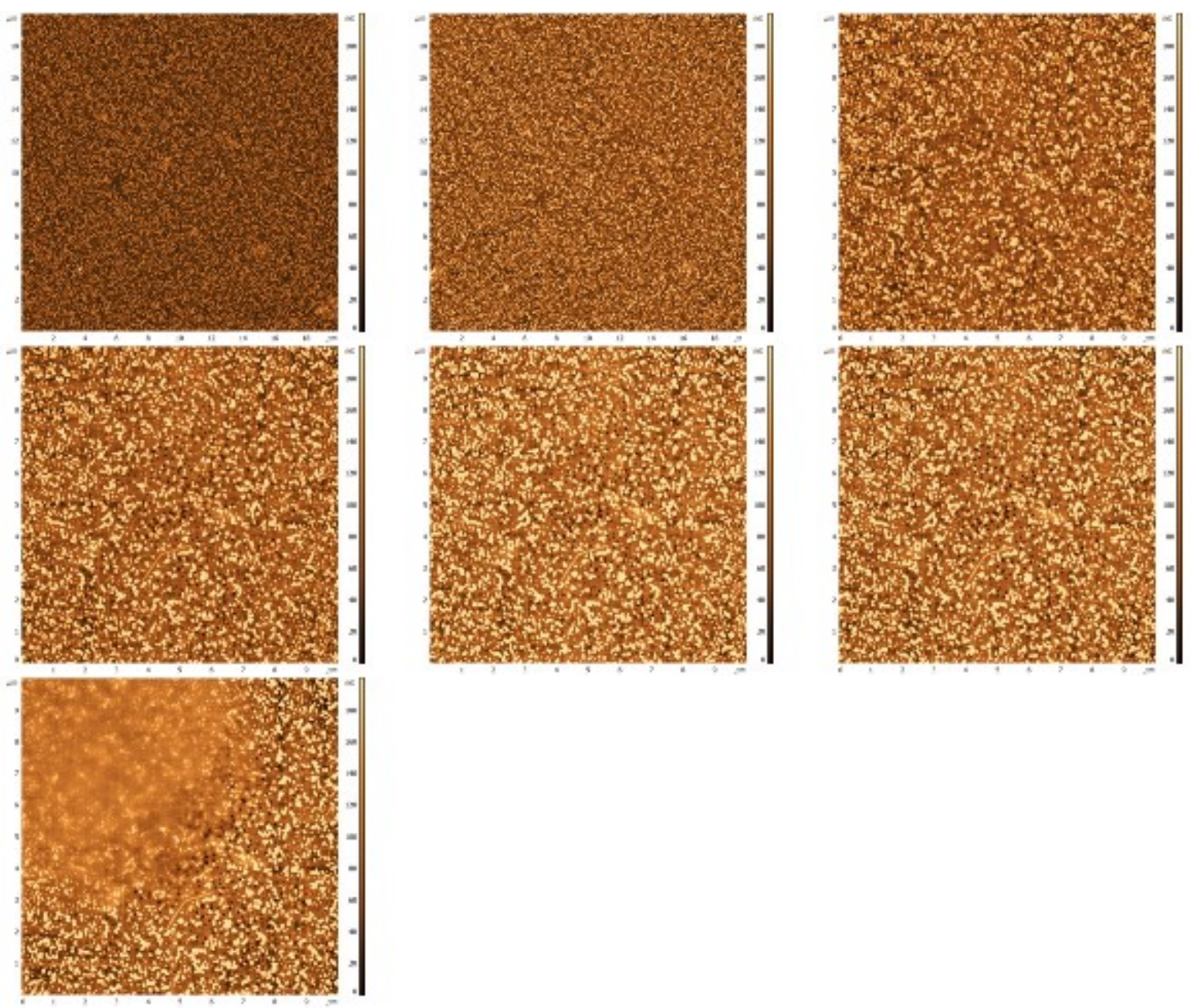

Figure 55: Selective Embedment on 1:1 mix at $458 \mathrm{~nm}$ then $568 \mathrm{~nm}$. Firstly, the substrate was exposed to $458 \mathrm{~nm}(22 \mathrm{~mW})$ for $5 \mathrm{~s}$, then $25 \mathrm{~s}$. The laser was switched to $568 \mathrm{~nm}(34 \mathrm{~mW})$ and the substrate was exposed for $5 \mathrm{~s}, 25 \mathrm{~s}, 2 \mathrm{~min} 35 \mathrm{~s}$, and $5 \mathrm{~min}$. Finally, the substrate was exposed to $568 \mathrm{~nm}(70 \mathrm{~mW})$ for 5 $\mathrm{s}$. The images are shown in chronological order. 


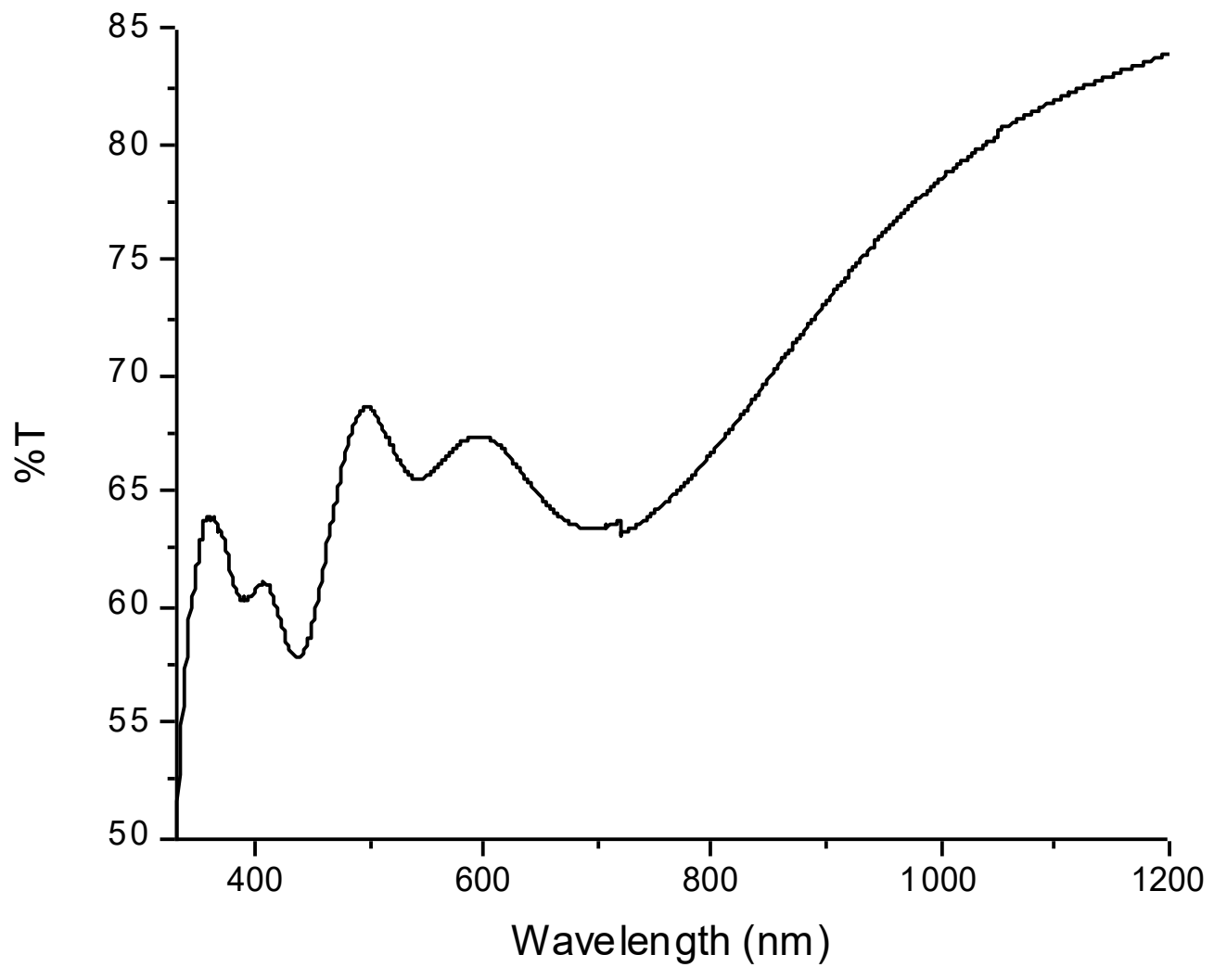

Figure 56: Transmission spectrum of 1:3 mix on PS showing the $D_{A g}, Q_{A g}, D_{A u}$ and $D-D$ plasmon modes. The D-D resonance transmits lower than $\mathrm{D}_{\mathrm{Au}}$. 